\title{
VICTIM AND PERPETRATOR PERSPECTIVES IN POST WORLD WAR II CONTEXTS: \\ INTERGROUP FORGIVENESS AND HISTORICAL CLOSURE IN EUROPE AND EAST ASIA
}

By

\author{
Katja Hanke
}

\author{
A thesis \\ submitted to the Victoria University of Wellington \\ in fulfilment of the requirements for the degree of \\ Doctor of Philosophy \\ in Psychology
}

Victoria University of Wellington

2009 


\begin{abstract}
The current thesis aimed to extend existing research on intergroup forgiveness by considering historical context as an important element. The clear victim and perpetrator roles in the European and East Asian post World War II settings provided the context for this research. Social representations of history provided the theoretical framework for four studies.

Study 1 employed a meta-analytical approach to explore the impact of contextual variables on interpersonal forgiveness across 13 societies. Based on Berry's ecocultural framework and Inglehart's affluence theory it was expected that socio-political, societal well-being and socio-economic variables are linked to interpersonal forgiveness. Significant differences in interpersonal forgiveness between the 13 societies emerged, which could be explained by conflict potential, socio-economic and socio-political context variables, societal peacefulness, societal well-being, and negative societal evaluations of historical calamities.

Study 2 explored conceptualisations of interpersonal and intergroup forgiveness using a qualitative approach with interviewees from Japan, Germany and the Philippines. Facets, antecedents and outcomes of forgiveness were identified as main themes. Differences in the relevance of forgiveness as a means of conflict resolution were revealed across cultures. An illuminating concept was identified and labelled as "historical closure", signifying an attitude towards historical issues as relevant or irrelevant to present and future relationships between groups.
\end{abstract}

Study $3 \mathrm{a}$ and $3 \mathrm{~b}$ included victim perspectives from three formerly victimized European nations (France, Poland, Russia) and three formerly victimized East Asian nations (China, Taiwan, Philippines). 
Study 3a examined differences in intergroup forgiveness across the six societies. Between-society differences were found. Chinese participants were less forgiving compared to French participants, pointing to the different historical contexts as an explanatory source. Study $3 b$ investigated the ability of historical closure and other group-based constructs to predict intergroup forgiveness. In both settings, historical closure was a consistent significant predictor and contributed to explain unique variance. A cross-level operator analysis revealed that political apologies by the perpetrator country during the last 20 years was negatively associated with intergroup forgiveness, indicating that external context related variables can contribute to explain intergroup forgiveness.

Study 4 investigated perpetrator perspectives from Japan and Germany, with focus on the cognitive and behavioural components of the willingness to make amends. Japanese and Germans differed significantly on the behavioural component: it was predicted by lack of closure in the Japanese sample; whereas in the German sample guilt and shame were positive predictors. Lack of historical closure consistently contributed to predicting the cognitive component of the willingness to make amends. Japanese experienced more guilt and shame feelings than Germans. Stronger national identification did not contribute as expected and had a reversed effect in Japan by being a positive predictor.

Historical closure is an intriguing concept, as it is a positive predictor for intergroup forgiveness among participants from formerly victimized nations, but a negative predictor for the willingness to make amends among participants from formerly perpetrating nations. This is an interesting interdependency in coming to terms with history: closure seems to be needed by victims to be ready to forgive, whereas the lack of closure for perpetrators seems to drive the willingness to make amends. 


\section{Acknowledgments}

This journey has been the most enjoyable and (until now "schlimmer geht immer") the most stressful time of my life.

This thesis would not have been possible without the academic, social and financial support of many people and institutions to whom I wish to express my deepest gratitude.

Thanks to my two supervisors, Prof James H. Liu and Dr Ronald Fischer, for their constant support and advice during the completion of my $\mathrm{PhD}$.

Special thanks to you Jim for giving me the tools to think outside the box, for being an inspiring mentor, for encouraging me, for supporting my ideas and for making this whole research possible. Thank you very much for your "kia kaha".

Thanks to you Ron for taking care of me during the initial start of my $\mathrm{PhD}$, for teaching me stats and for giving me advice when it was crucial.

I would like to thank the BRCSS network (Building Research Capabilities in the Social Sciences) for supporting this thesis by providing research funds. Furthermore, I would like to thank Victoria University for awarding me with a $\mathrm{PhD}$ submission scholarship in 2009.

Thank you, Prof Etienne Mullet (Institute of Advanced Studies, France), Prof John Duckitt (University of Auckland, New Zealand) and Prof Colleen Ward (Victoria University of Wellington, New Zealand) for examining my thesis.

Many, many thanks to you Pip Collie for proofreading my thesis in such a fast pace that I couldn't believe my own eyes. Your comments helped me a lot. Thanks for your patience and your accessibility even though you work fulltime and do volunteer work.

To the staff members at VUW - thank you very much for helping to deal with bureaucracy, and for your kind advice: Ngaire Lavery, Jebi Jayapalan, Maria Goncalves-Rorke, and Shona de Sain.

For enlightening discussions and sharing their interest in this topic, I would like to express my gratitude to Prof Marilynn B. Brewer, Prof Michael H. Bond, and Prof Ype Poortinga.

My sincere gratitude goes to my collaborators for their remarkable help in coordinating and collecting the data and translating my questionnaires. Without the help of following people this research would not have been possible (in alphabetical order): Prof Tomohide Atsumi, Dr Michal Bilewicz, Diana Boer, Prof Hans-Peter Erb, Prof Ilya E. Garber, Prof M. Cecilia Gastardo-Conaco, Prof Denis Hilton, Prof Li-Li Huang, Ilka Reuter, Dr Ko-ichi Suwa, Dipl.-Psych. Deborah F. Thoben, Melanie Vauclair, and Dr Feixue Wang. Thank you.

Thanks to almost 1500 participants for sharing their views on forgiveness and on making amends. 
I don't know how to express my gratitude towards the cross-cultural lab. What an awesome bunch of wonderful people! Thank you so much for your emotional, social support, for sharing and discussing academic ideas, and just for being who you are - you are my NZ family. I will really miss you, but I am sure we will meet again. Thanks to all of you!

Thank you very much to you my friends in Germany and in New Zealand for their constant encouragement and support - no matter where they were. Special thanks to Katja Liebau for helping me out on many different occasions.

Finally, I wish to thank to family. Thank you for your firm belief in me and your lovely support. Thanks to my mom who kept me motivated and made me laugh. Thanks to my dad who kept me updated about what is going on in the world ("What?! You don't know that?"). I cherish our phone calls. Thanks to my brother and his family. I am looking forward to meeting my nephew. I love you from the bottom of my heart.

At last, I would like to express my deepest gratitude to Diana - you are the best that happened to me! Without you I would not be here, I would not have been able to endure this. Dito! Ich Dich noch viel mehr!

"Man sieht nur mit dem Herzen gut. Das Wesentliche ist für die Augen unsichtbar!“ (Der kleine Prinz - Antoine de Saint-Exupéry)

I would like to dedicate my thesis to all people who are not as fortunate as we are to live a life free of conflict, of violence, and of human suffering.

I care about you. 
ABSTRACT ................................................................................................................................ II

ACKNOWLEDGMENTS ............................................................................................... IV

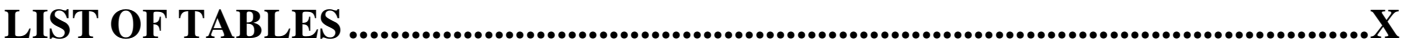

LIST OF FIGURES ........................................................................................... XI

CHAPTER 1

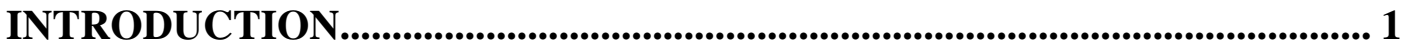

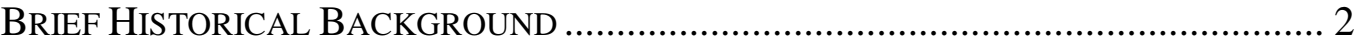

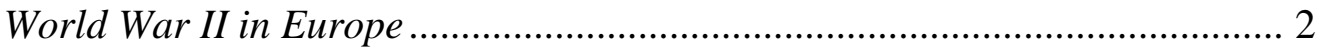

World War II in East Asia .......................................................................... 4

AIM AND OUTLINE OF THE CURRENT THESIS ................................................... 6

\section{CHAPTER 2}

INTRODUCTION TO INTERGROUP FORGIVENESS ............................... 9

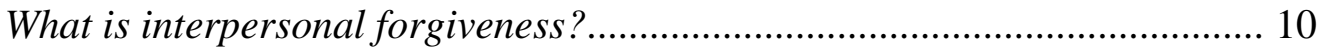

What is intergroup forgiveness? .................................................................. 12

What is the difference between forgiveness at the individual and at the group

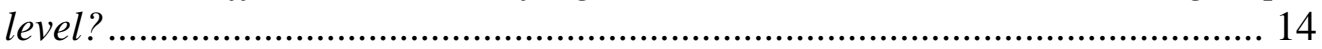

Working definition of intergroup forgiveness .............................................. 15

THE PSYCHOLOGY OF INTERGROUP FORGIVENESS ............................................. 16

Social Identity Approach and the Contact-Hypothesis................................. 16

Social Identity Approach: Social Identity Theory (SIT) and Self-

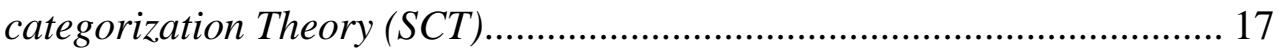

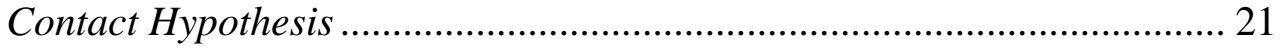

Empirical research on intergroup forgiveness ......................................... 23



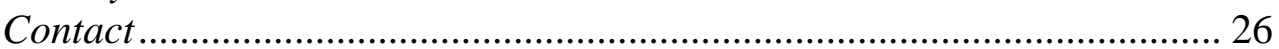

Collective guilt and collective guilt assignment ....................................... 28

Collective shame ..................................................................................... 30

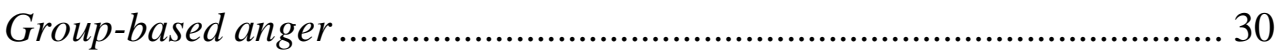

Concluding remarks about SIT/SCT based intergroup forgiveness research

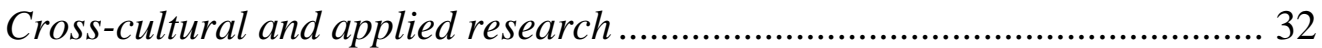

RESEARCH GAPS AND CRITICAL EVALUATION OF PREVIOUS RESEARCH................ 33

SOCIAL REPRESENTATIONS THEORY (SRT) …................................................ 35

Social representations of history and intergroup forgiveness ....................... 39



CHAPTER 3

STUDY 1: SOCIO-ECONOMICAL AND SOCIO-POLITICAL

CONTEXTUAL CORRELATES OF INTERPERSONAL FORGIVENESS -

A META-ANALYTICAL APPROACH ACROSS 13 SOCIETIES .................. 46



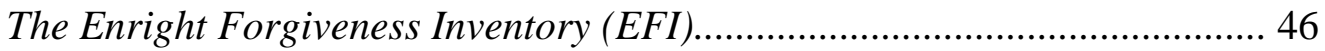

A cross-national meta-analytical approach.................................................. 52

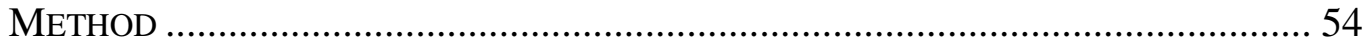



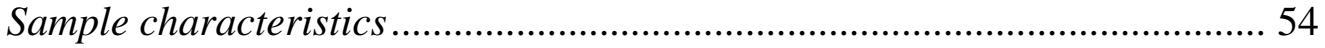


Psychological variables, socio-economical and socio-political context variables

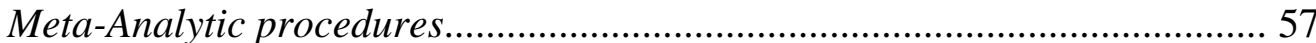

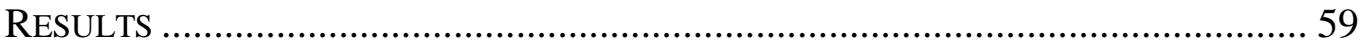

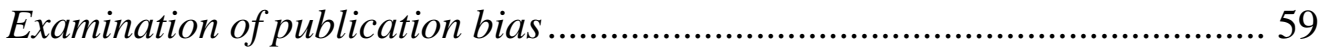

Interpersonal forgiveness across 13 societies .............................................. 59

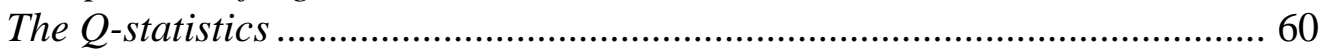

Explanation of differences in exploring possible moderators at the study level

Correlations at the society level

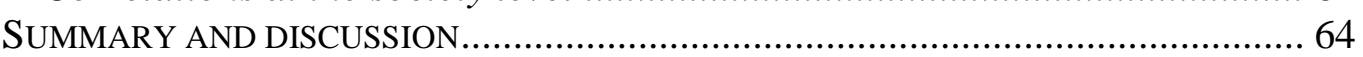

\section{CHAPTER 4}

\section{STUDY 2: QUALITATIVE EXPLORATION OF INTERGROUP

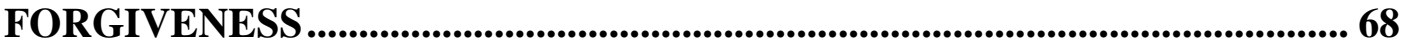

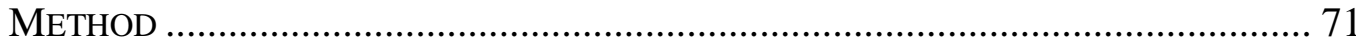

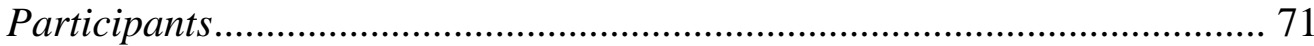

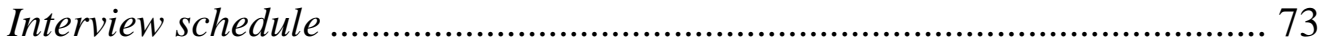

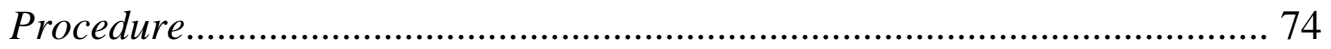

Analytical strategy: Retroductive thematic analysis................................... 74

RESULTS AND DISCUSSION: CONTEXTUALIZED INTERGROUP FORGIVENESS ......... 76

Facets of Intergroup Forgiveness - distinguishing interpersonal from

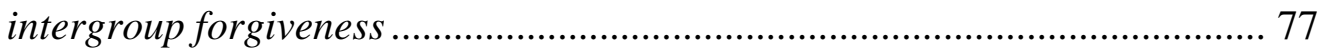

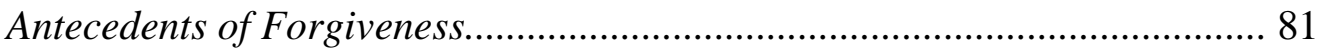

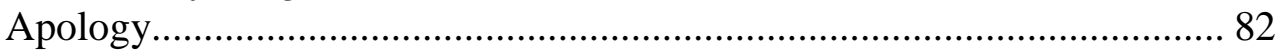

Collective emotions and memories .................................................... 90

Representation and historical closure with the past................................ 93

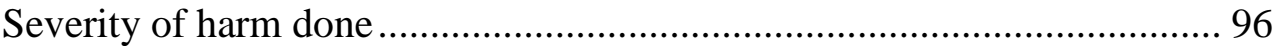

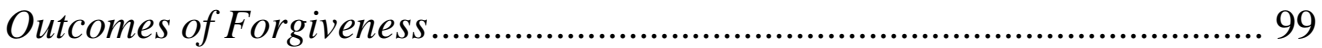

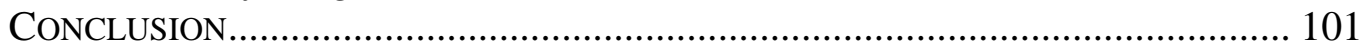

\section{CHAPTER 5}

\section{STUDY 3: VICTIM PERSPECTIVES - INTERGROUP FORGIVENESS ACROSS EUROPEAN AND EAST ASIAN CONTEXTS ................................ 107}

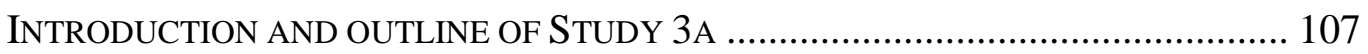

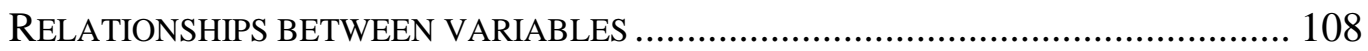

Historical closure and costs of forgiveness .............................................. 108

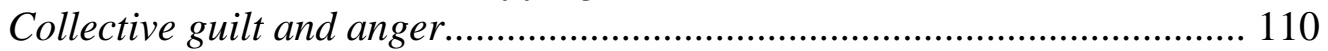

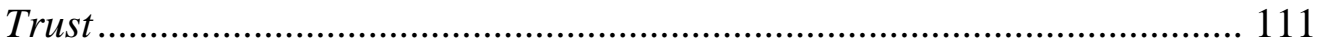

The role of identification with one's national group. ................................ 112

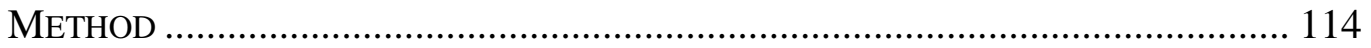

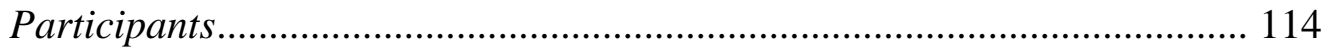

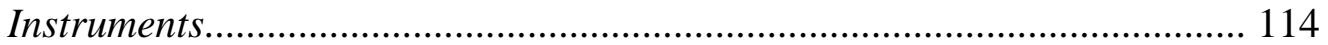

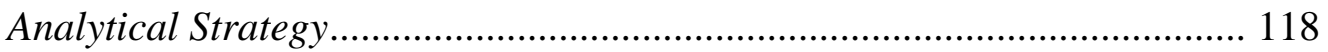

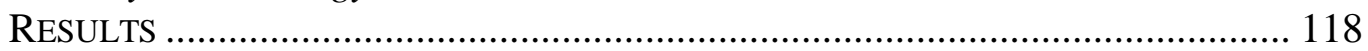

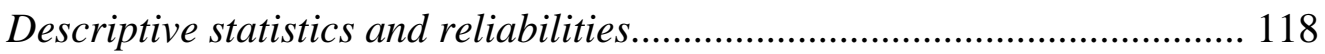

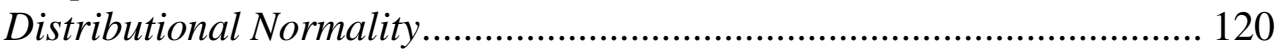

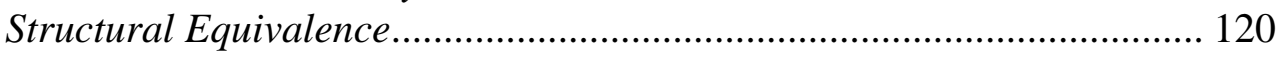

Between society differences on intergroup forgiveness .............................. 121

Summary of between society differences on other variables .................... 121

Relationships among concepts ................................................................ 122 
Multidimensional Scaling (MDS): Depicting underlying dimension of meaning

Similarity of correlation coefficients .................................................... 126

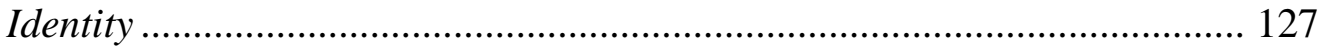

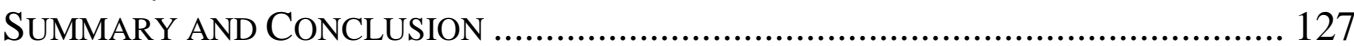

STUDY 3B: INTERGROUP FORGIVENESS AND THE HISTORICAL CONTEXT: MULTIPLE HIERARCHICAL REGRESSIONS AND CROSS-LEVEL OPERATOR ANALYSIS (CLOP) 128

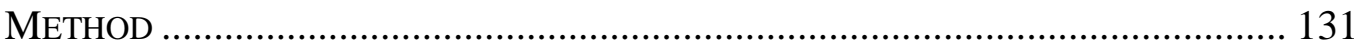

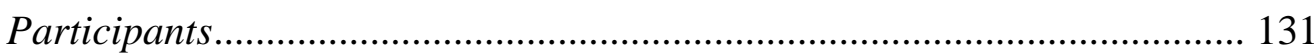

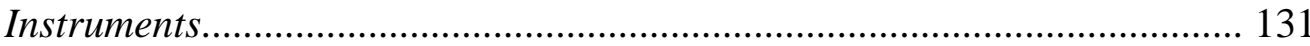

Society-Level Contextual Variables ........................................................... 131

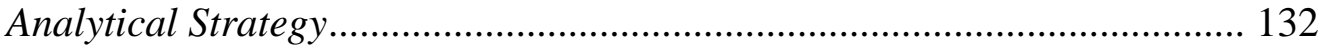

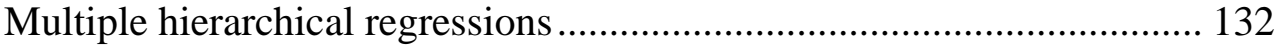

Cross-level operator analysis (CLOP) ……......................................... 132

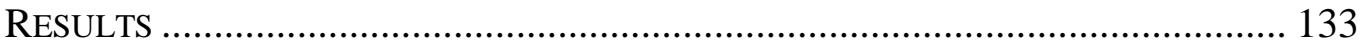

Multiple Hierarchical Regressions ........................................................... 133

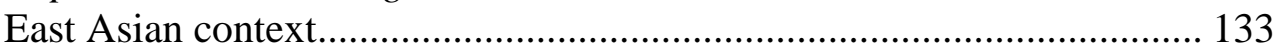

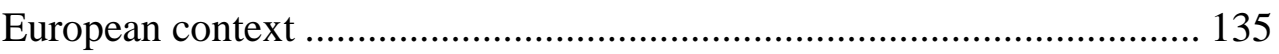

Cross level operator analysis (CLOP) .................................................... 136

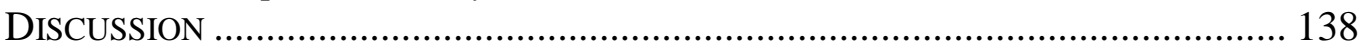

\section{CHAPTER 6}

STUDY 4: PERPETRATOR PERSPECTIVES - GERMAN AND JAPANESE WILLINGNESS TO MAKE AMENDS.................................................. 145

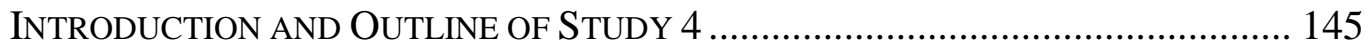

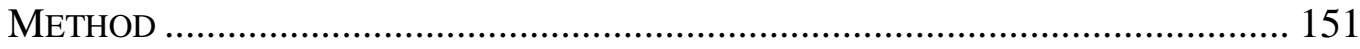

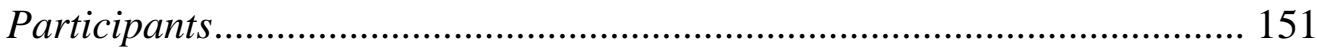

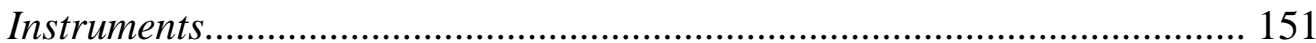

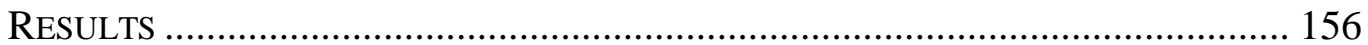

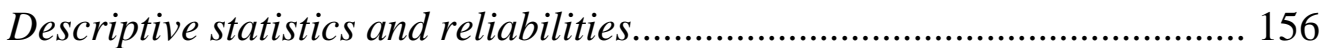

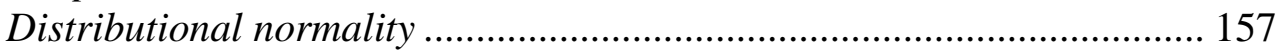

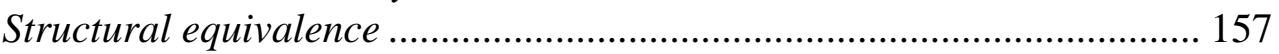

Description of differences between Germany and Japan ....................... 158

Interrelationships between concepts.................................................... 163

Similarity of correlation coefficients ................................................... 165

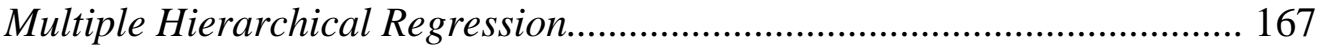

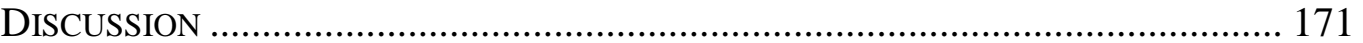

CHAPTER 7

GENERAL DISCUSSION AND CONCLUSIONS .......................................... 176

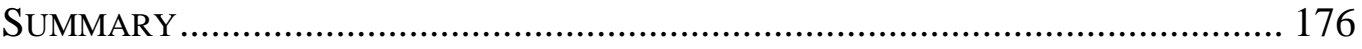

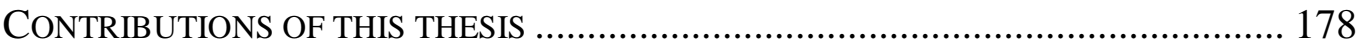

Theoretical implications of the current thesis ............................................ 180

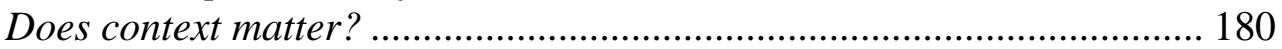

Predictors of intergroup forgiveness and willingness to make amends ... 185

What happened to national identification? ................................................ 188

"Shame" versus "Guilt" Cultures ............................................................ 190

Intergroup forgetting versus intergroup forgiveness ............................... 191

Revisiting the process of intergroup forgiveness ..................................... 193

LIMITATIONS AND SUGGESTIONS FOR FUTURE RESEARCH …............................. 194

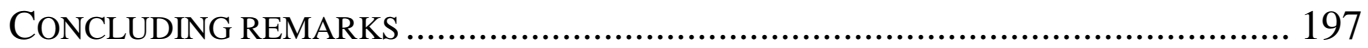




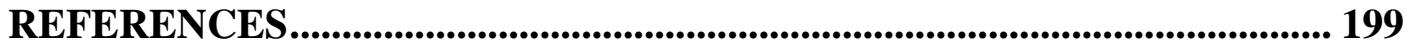

APPENDICES ................................................................................................... 222

APPENDIX A - SEMI-STRUCTURED INTERVIEW SCHEDULE ................................ 222

APPENDIX B - ADDITIONAL INFORMATION FOR CHAPTER 5 …......................... 223

APPENDIX C - ADDITIONAL INFORMATION FOR CHAPTER 6 ............................. 236 


\section{List of Tables}

Table 3.1

Country-level indicators and corresponding country scores (Study 1) .57

Table 3.2

Mean levels (effect sizes) per society based on mixed-effects model (Study 1)

Table 3.3

Correlation between EFI means and Well-being Index, Democracy Indicators, Peace Indicators, Socio-Economic Indicators, and Historical Calamities at the country level (Study 1)

Table 5.1

Sample description $(N=1197$, Study $3 a)$

Table 5.2

Means, Standard Deviation, Cronbach's alpha, F-values (society as fixed factor)

(Study 3a)

Table 5.3

Multi-group CFA fit indices for intergroup forgiveness, historical closure, and costs of forgiveness (Study 3a)

Table 5.4

Intercorrelations between Scales for overall Sample $(N=1197$, Study 3a)

Table 5.5

Multiple Hierarchical Regression, East Asian context $(N=624$, Study $3 b)$.

Table 5.6

Multiple Hierarchical Regression, European context $(N=573$, Study 3b)

Table 5.7

Summary of hierarchical regression analysis of variables predicting intergroup forgiveness using cross-level operators (CLOP) (Study 3b)

Table 6.1

Sample description for Japan $(N=132)$ and Germany $(N=134)$ (Study 4)

Table 6.2

Means, Standard Deviation, Cronbach's alpha, t-values (Study 4) .156

Table 6.3

Intercorrelation Matrix for all scales in Japan $(N=132)$ and Germany $(N=134)$

(Study 4)

Table 6.4

German versus Japanese context willingness to make amends (cognitive component), standardized $\beta$-weights (Study 4)

Table 6.5

German versus Japanese context predicting willingness to make amends (behavioural component), standardized $\beta$-weights (Study 4) 


\section{List of Figures}

Figure 3.1 Funnel Plot of the effect sizes (EFI-mean) and sample size (Study 1)..59

Figure 4.1 Summary of identified themes (Study 2).........................................102

Figure 5.1 Two-dimensional MDS representation overall (Study 3a) ................125 


\section{Chapter 1}

\section{Introduction}

One of the most challenging political endeavours in the post-Cold War age is how to deal with the aftermath of extreme conflicts and their repercussions, with the aim of developing sustainable peace. Despite continual attempts to foster peaceful coexistence, the world we live in looks very different from living in a world free of intergroup violence (Hartwell, 2005; Ergüner-Tekinalp, 2007). Minow (1998) asks whether the twentieth century will mostly be remembered for mass atrocities. Undoubtedly, the twentieth century was one of the bloodiest centuries in human history with gross human rights violations, and genocides beyond imagination (McCullough, Thoresen, \& Pargament, 2000).

The present also rears its ugly head with global terrorism, interethnic and intergroup violence and other gross human rights violations proving once more the inability of human kind to solve conflicts peacefully. The origins of many conflicts today instigate from past conflicts between nations or groups and are plainly new variations of old conflicts (Hartwell, 2005; Staub, 2000). Furthermore, the interpretation of past conflicts is often used by elites to justify actions against specific groups or nations, exacerbating boundaries between groups (Hilton \& Liu, 2008; Liu \& Hilton, 2005).

In this context, it seems surprising that the investigation of the concept of intergroup forgiveness in times of past and present injustices, and unclosed disputes over past conflicts is not well developed (Roe, 2007). Overall, little is known about the process of forgiveness between groups or nations engaged in conflicts (either at the symbolic or physical level) or groups still suffering from past conflicts (Roe, 2007). Interdisciplinary perspectives have promoted 
forgiveness as a proactive, reasonable means to resolve conflicts and work towards reconciliation. The forgiveness process focuses on creating and enhancing conditions to ensure the empowerment of victims and to provide an opportunity to release victims from victimhood (Amstutz, 2005; Digeser, 2001; Minow, 1998; Oliner, 2005; Shriver, 1995). Overall, forgiveness between nations and groups sparks a glimpse of hope for the ability to transform deep-seated conflicts and disrupt cycles of violence, which may lead to genuine and sustainable reconciliation.

The post World War II (WW II) context is a crucial historical context that still has an influence on younger generations. For instance, just recently a trial against a Nazi war criminal has begun in Germany. Since the German legal system proposes that war crimes, such as mass murder and assistance to mass murder during WW II will never be subject to a limitation period, criminal charges against WW II war criminals can be pressed any time (Frei, 2009). Frei (2009) argued that Germany owes it herself and her victims to bring justice to war crimes committed during WW II. On the other hand, Japan has in recent years had to defend herself several times from the charges that Japan is engaged in "whitewashing" past war crimes in officially sanctioned high school textbooks (e.g. Schneider, 2008). Intergroup forgiveness research in the context of such a crucial event as WW II and the comparisons between the European and East Asian context can advance the overall understanding of forgiveness.

\section{Brief Historical Background}

\section{World War II in Europe}

The Holocaust encapsulates the pure evil of WW II and is a symbol of atrocities, which remains a burden for present-day Germans. This is evident in 
relatively low historical pride where only $8 \%$ Germans replied "yes" to the questions concerned about historical pride compared to a $34 \%$ general mean across 23 European, Asian and American Nations (UNESCO, 2000; cited in Hilton \& Liu, 2008). Hauss (2003) noted that every German government since the formation of the federal republic in 1949 has aimed to establish good relationships with Israel and with her neighbours, guided by the consequences of this war. Furthermore, there have been active efforts to redress the harm inflicted in the past resulted in to date $\$ 70$ billion of compensations and reparations payments made directly to victims of slave labour, Holocaust survivors and their descendents (Oliner, 2008).

Willy Brandt was the first German chancellor who made a public expression of repentance to Jewish people by kneeling in front of the Warsaw ghetto memorial to weep for the victims. His efforts of rapprochement and reconciliation opened up a new dialogue in the East-West politics in the 1970s and were internationally acknowledged with the Nobel Peace Prize (Oliner, 2008). In 1985 Federal president von Weizäcker recognized the unspeakable horror of the Holocaust as always being a part of German history in a renowned speech. He stressed the importance of remembering regardless whether Germans bear guilt or not, no matter whether they are old or young, as everyone is affected by the consequences (Bindenagel, 2002). Oliner (2008) considers the numerous attempts of apologies from German governments made since 1949 as successful, because they have taken responsibility for their harmful actions in the past. These apologies have come alongside coherent actions and policy implementations towards reconciliation.

Furthermore, the German educational system has integrated coverage of the rise and fall of the Third Reich in its educational syllabi. Generally, Germany and her neighbours share the same historical facts and agree on them. This is a result of 
long-term textbook commissions starting in 1951 between France and Germany, followed by Poland, the Czech Republic, and Israel, involving historians and teachers. The textbook commissions aimed for an international understanding, and reduction of enemy stereotypes (e.g. Ruchniewiecz, 2005; Viedt, 1993).

The victims of Germany's war crimes were primarily Jews, Gypsies, and other "deviant" groups, such as homosexuals or people with disabilities. However, in terms of nationalities, Poland and Russia suffered the most, followed by France. In Poland three million people lost their lives, in Russia 22 million, and in France almost half a million excluding Jewish victims. After WW II the era of the Cold War started, and this can explain the relatively fast attempts of rapprochements between France and Germany and the relatively slower attempts at rapprochement with Poland and Russia.

\section{World War II in East Asia}

The Japanese political elite have been very "inept at political mea culpas" (Oliner, 2008, p. 84, italics in original), as they have been divided over issuing apologies for the unspeakable crimes committed as a result of Japanese militarism in the 1930s and 1940s (Hauss, 2003). The efforts of apologizing for and acknowledging the past harm done by Japan are far from ideal, especially in comparison to Germany's efforts to make amends for their role in WW II (Hein \& Selden, 2000). People in many East Asian countries have suffered at the hands of imperial Japan, who inflicted cruelty and war crimes that were similar in magnitude to the crimes committed by Nazi Germany (Liu \& Atsumi, 2008; Oliner, 2008). Victims of Japan during WW II were mostly Chinese, Koreans, and Filipinos among other East Asian nations. Twenty million Chinese, almost half a million Koreans, and one million Filipinos lost their lives during WW II. 
The Nanjing Massacre and the Comfort Women Issues (forced prostitution) are symbols of this era for victimized East Asian nations. The victims of these war crimes were mostly Chinese, Filipinos, Indonesian, Malaysians, and Koreans to mention just a few. The Japanese government have attempted to apologize (Hauss, 2003; Oliner, 2008; Yamazaki, 2006). However, these apologies were mostly ineffective and were characterized as being personal rather than political apologies. These apologies often deepened the anger of victims (Oliner, 2008; Yamazaki, 2006), because they were perceived as hypocritical and deceptive due to concurrent non-acknowledgement and non-admittance of the atrocities committed during WW II in some textbooks approved by the Ministry of Education (Liu \& Atsumi, 2008).

Japan's crimes during WW II were portrayed as minor incidents in some Japanese textbooks, if mentioned at all (Hein \& Selden, 2000). This reluctance to reveal the truth by some segments of the political elite has led to a refusal and nonappreciation of issued apologies made by Japan throughout East Asia. Many survivors of those crimes and their descendents continue to hold grudges against Japan. The so-called "Rape of Nanking" where perhaps as many as 300,000 men, women, and children were killed in 1937-38 (Chang, 1998) remains unresolved and is factually contested, leaving Chinese victims and their offspring with continued anger (Liu \& Atsumi, 2008). Filipino and Korean former comfort women who were used as sex slaves during WW II still demonstrate in front of the Japanese embassy in Manila and Seoul respectively for an official apology and compensation for their suffering. The survivors fear that their suffering will be forgotten once they die (Pennebaker, Páez, \& Rimé, 1997). So far, the Japanese government has refused to come clear about its own past. Therefore, the apologies it has issued are perceived as half-hearted and not trustworthy, and may have made matters worse rather than better (Oliner, 2008). 
Have these differing traditions of dealing with a dark past influenced the process of peacemaking and intergroup reconciliation in Europe and East Asia? The focus of this particular thesis is on the applicability of the concept of forgiveness in dealing with a troubled past between groups.

\section{Aim and outline of the current thesis}

The aim of the current thesis was to investigate the relevance of the societal and historical context for intergroup forgiveness, allowing further predictions and explanations of differences in the propensity to forgive and the willingness to make amends for past injustices respectively. The post WW II relations in the European and in the East Asian context take centre stage for this research. This is a novel undertaking, since previous research has focused on within-group conflicts where the victim and perpetrator roles were intertwined (e.g. Northern Ireland). In addition to the European context, the current thesis attempted to shed some light on the post WW II relations between Japan and her formerly victimized East Asian neighbours, since the East Asian context has been neglected for too long.

The current thesis consists of four studies reported in Chapters 3 to 6. The thesis closes with a general discussion and conclusion in Chapter 7. In Chapter 3, Study 1 investigates in a cross-national meta-analytical approach if and how societies differ on interpersonal forgiveness. Study 1 aimed to reveal whether contextual/societal factors influence interpersonal forgiveness. Subsequently, sources of explanations for observed differences are examined and associations between socio-economic, socio-political, peacefulness of society and societal evaluations of historical calamities and interpersonal forgiveness across 13 societies are investigated.

Study 2 in Chapter 4 provides an insight into how Japanese, Filipino and German participants differentiate between interpersonal and intergroup forgiveness 
using a qualitative approach. Study 2 explored how participants articulate conditions and relevance of forgiveness for conflict resolution. The identification of key concepts in the intergroup forgiveness process provided new concepts (historical closure in particular) that are investigated in Chapters 5 and 6.

Chapter 5 examines victim perspectives from three formerly victimized European nations (France, Poland, Russia) and three formerly victimized East Asian nations (China, Taiwan, Philippines). Study 3a tested whether the historical context in Europe and East Asia influences the forgiveness process. If this impact occurs, it should be reflected in substantial differences in the outcome variable of intergroup forgiveness, which are also associated with mean differences between nations and contexts in variables such as collective guilt, and historical closure. These variables and the convergent validity of historical closure and costs of granting forgiveness were assessed to investigate these claims.

Study $3 b$ examined the impact of established psychological antecedents (such as guilt and trust) and new concepts (such as historical closure) on intergroup forgiveness in post World War II contexts in Europe and East Asia using regression analysis. The unique contribution of the new concepts are assessed and discussed in Chapter 5. Notably, Study $3 b$ employed a statistical technique called cross-level operator analysis to tackle the influence of context variables. In this analysis, external contextual variables (i.e. number of issued public apologies during the last two decades) were assessed in terms of their contribution to explain variation in intergroup forgiveness at the individual level. Contextual variables that reflect the historical context were chosen.

Chapter 6 examines perpetrator perspectives from Germany and Japan. It is proposed that the historical context influences the process of being willing to make amends. Making amends complements the forgiveness process, since it is argued 
that the overall forgiveness process includes both a victim and perpetrator side. The impact of the historical context may reflect crucial differences between Japanese and German participants on the outcome variable of willingness to make amendments. Furthermore, established psychological antecedents and historical closure among other new concepts (such as face concerns) are investigated in their ability to predict the willingness to make amendments. The unique contribution of the new concepts to explain variance in the willingness to make amends is examined and evaluated.

Finally, Chapter 7 summarizes, discusses and integrates major findings. The chapter deals with the limitations of the current research and suggests future research. It closes with concluding remarks. 


\section{Chapter 2}

\section{Introduction to intergroup forgiveness}

\section{Summary of developments in forgiveness research}

The concept of forgiveness has been of interest and encouraged for centuries by major religions (McCullough, Pargament, \& Thoresen, 2000). Before philosophers and other scholars have gotten interested in this topic, theologians devoted their scholarship to understanding the age-old experience of forgiveness. Forgiveness' strong religious ties caused neglect in scientific research. Due to a high reluctance to mix religious and scientific topics, serious attempts to study forgiveness psychologically did not start until the mid 1980s. Furthermore, research has been restricted to understanding interpersonal forgiveness (see for an overview Worthington, 2005).

The scientific psychological research of interpersonal forgiveness can be categorized into three sequential waves. First, clinicians, therapists, and family counsellors were interested in applying forgiveness to interventions to foster healing among their clients (e.g. Smedes, 1984). Second, developmental psychologists (Enright \& the Human Development Group, 1989) started researching the development of forgiveness reasoning, and personality psychologists began researching forgiveness as a trait (e.g. Kamat, Jones \& Lawler-Row, 2006). Third, health psychologists commenced researching physical health outcomes (e.g. Lawler, Younger, Piferi, Billington, Jobe, Edmondson, \& Jones, 2003) and social psychologists initiated studies on how forgiveness occurs in everyday interactions including a recent interest in studying interpersonal forgiveness cross-culturally (e.g. Takaku, 2001). The latter field of study remains under researched (see Sandage \& Williams, 2005). 
Studies have addressed questions such as how forgiveness as a process is able to heal hurt caused by the harm done, and its power to bring people together again (Fincham, Jackson, \& Beach, 2005). This literature generally incorporates an analysis of the benefits of forgiveness and the drawbacks of not forgiving in interpersonal (but not intergroup) settings (for an overview see McCullough et al., 2000; Worthington, 2005). For example, forgiveness has been proposed as an intervention method and has been applied in therapeutic settings. An important finding of this work is that forgiveness is positively related to an individual's psychological well-being (Karremans, van Lange, Ouwerkerk, \& Kluwer, 2003; Rye, Folck, Heim, Olszewski, \& Traina, 2004), greater life-satisfaction (Poloma \& Gallup, 1991) and happiness (Maltby, Day, \& Barber, 2005).

Although a reasonable amount of research has addressed and explored the concept and process of forgiveness, many questions remain unanswered in the domain of interpersonal forgiveness (Worthington, 2005). However, Worthington noted that despite the tendency for each researcher to state that there is no consensus about the definition of forgiveness, "there seems to be a near consensus" (p. 557). In the following section, I define and distinguish the two concepts of interpersonal and intergroup forgiveness.

\section{What is interpersonal forgiveness?}

Many scholars argue that forgiveness is distinct from excusing, exonerating, justifying, condoning, pardoning, or reconciling (for a summary see Worthington, 2005). Worthington argues that while the landscape of various forgiveness definitions seems confusing, forgiveness tends to be conceptualised in two ways: one definition focuses on the intrapersonal experiences of forgiveness, and the other one considers the interpersonal process of forgiveness.

An often-cited definition of forgiveness at the interpersonal level is: 
"Forgiveness is a person's decision to substantially reduce or even abandon resentment, negative judgment, and indifferent behaviour toward another person who unjustly injured him or her, while fostering compassion, generosity and even love toward the other person" (Enright, Freedman, \& Rique, 1998, pp. 46-47).

Worthington and McCullough (1999) defined interpersonal forgiveness as a positive change in emotions, cognitions and behaviours toward the transgressor party. That is, when people forgive, their emotional, cognitive and behavioural responses towards people who have offended or injured them become more positive and less negative. Hargrave and Sells (1997), who come from a therapeutic background, define forgiveness as an unfolding process in which the victim and the victimizer rebuild trust and promote an open dialogue about the offence with the aim of rebuilding the relationship. Scobie and Scobie (1998) regard forgiveness as "a prosocial facilitator" pointing to the relational aspects involved in rebuilding the relationship as the outcome of the forgiveness process. McCullough and colleagues (2000) conclude that existing definitions suggest that forgiveness is an "intraindividual, prosocial change toward a perceived transgressor that is situated in a specific interpersonal context" (p. 9).

Later, Worthington (2005) summarized definitions of forgiveness and distinguished three types of forgiveness: decisional forgiveness, experience of forgiveness and the process of interpersonal forgiveness. The focus of this thesis is on the process of intergroup forgiveness (see Chapter 1). According to Worthington (2005), the process of interpersonal forgiveness is defined in the following way. Forgiveness involves interactions surrounding a transgression. These interactions include various micro-processes (e.g. seeking forgiveness or rejecting the need of forgiveness by the offending party) and cognitions (e.g. attributions) as well as emotions (victimized party's expression of emotional 
forgiveness). Furthermore, the process of forgiveness is influenced by whether there is a desire to continue the relationship. Worthington further refers to the societal level and acknowledges that the historical context and salient identities may have an impact on the interpretations of events (Worthington, 2005).

\section{What is intergroup forgiveness?}

The significance of context within the mentioned definitions is unclear, although Worthington (2005) did acknowledge that context influenced the forgiveness process. The stated definitions emphasise the role of the individual and intrapersonal changes. The definitions paid little attention to how transgressions and the forgiveness process affect people as a group. Indeed, there is no clear definition of intergroup forgiveness in the literature. The seeds for the conceptualisation of intergroup forgiveness in previous research have come from definitions of interpersonal forgiveness (e.g. Hewstone, Cairns, Voci, McLernon, Niens, \& Noor, 2004). Some conceptualisations of interpersonal forgiveness might be applicable for forgiveness processes between groups (e.g. role of the situation, severity of harm done), but others may be not appropriate (e.g. independence from the context).

Most previous research acknowledges that there are substantial differences between interpersonal and intergroup forgiveness (e.g. Hewstone et al., 2004). Intergroup forgiveness is predominantly defined based on Enright's and the Human Development Group $(1991,1998)$ definition (quoted above), or a mix of definitions based on McCullough and colleagues' (2000) and Enright's understanding of forgiveness applied to the intergroup level.

Strictly speaking, the definitions provided by Enright and the Human Development Group $(1991,1998)$ are not applicable in intergroup contexts, as they are mostly concentrated on the individual and focus on unilateral, intrapersonal 
change which is independent from any actions of the former perpetrator. Furthermore, according to Enright and the Human Development Group (1991), the most developed form of forgiveness transcends social contexts (Andrews, 2000). However, the context is a necessary part to understand interpersonal and even more so intergroup forgiveness (Andrews, 2000).

Andrews' (2000) conceptualisation of negotiated forgiveness and Montiel's (2000, 2002) conceptualisation of constructive socio-political forgiveness are useful for the definition of intergroup forgiveness. Forgiveness is based on fulfilling preconditions before forgiveness is granted - although fulfilling these preconditions does not guarantee that forgiveness will take place. Applied to the intergroup context, negotiated forgiveness involves the idea of accountability and a dialogue between the victim and perpetrator party that may further understanding and trust (Mellor, Bertherton, \& Firth, 2007).

Montiel (2000) stressed the existence of qualitative differences between intrapersonal, interpersonal and socio-political forgiveness. From her point of view, socio-political forgiveness involves large pluralities of individuals and not human individuals (intrapersonal) or dyads (interpersonal). Furthermore, she argued that socio-political forgiveness deals with social healing that goes beyond intrapersonal healing. Moreover, she argues that an increase of subjective intrapersonal wellbeing does not automatically transform into social healing. Montiel (2000, p. 95) regards this assumption as "an analytical error of those who take a highly individualistic stance". Forgiveness at the intrapersonal and interpersonal level is a private experience whereas socio-political forgiveness is a public one (Montiel, 2002). The public and external character of "public forgiveness requires a sensitive handling of the historical, cultural, and political contexts of both conflicting groups" (Montiel, 2002, p. 271). 
What is the difference between forgiveness at the individual and at the group level?

Forgiveness at the individual level can be described as a 'virtue', since it is a moral response to transgressions (Enright \& Rique, 2000). It can also be described as a process depending on the situation and the relationship between the victim and perpetrator (e.g. this is more likely for people who have ongoing contact with each other, such as life partners). Furthermore, it entails a conscious decision, but not necessarily involving both parties. Forgiveness at the individual level allows victims to gain a sense of closure, reflected in reported increase in wellbeing and decrease in anxiety. Therefore, the benefits of forgiveness at the individual level are many, including a release from a damaging cycle of negative emotions and actions.

As aforementioned, the boundaries between forgiveness at the individual and group level are blurred in the literature. First and foremost, the fact that forgiveness has been conceptualized by scholars from different disciplines as a purely interpersonal affair only involving the victim and the perpetrator directly connected to an offence, or even as a solely intrapersonal process, has led to the impression that forgiveness has no place between groups or even between nations.

The notion of forgiveness as a strictly interpersonal process does not consider that many harmful acts in social life involve collectives (Minow, 1998; Thomas \& Garrod, 2002). Particularly in war and other contexts in which severe human rights violations occur, harm done is not only targeted at individuals but at societies as a whole or what societies represent (Neto, Pinto, \& Mullet, 2007a; Thomas \& Garrod, 2002). Therefore, one of the major differences between forgiveness at the individual and at the group-level is that offences in interpersonal contexts can be directly attributed to an individual, whereas in intergroup contexts it is difficult to ascribe an offence to any specific individual (Enright $\&$ the Human 
Developmental Study Group, 1991; Tavuchis, 1991). Hewstone and colleagues (2004) noted that the link between forgiveness and trust suggests that it must be easier to forgive an individual than a group since it is easier to trust an individual. Furthermore, they suggested that forgiveness is easier if there is an apology or acknowledgment of the harm done, and that a lack of public acknowledgment can fuel grudges and grievances. Moreover, it may be easier to gain closure and depart from the past between two individuals, as it is possible to break-up a relationship that is no longer desired, or to effectively intervene if a continued relationship is desired. This complexity makes it difficult to define forgiveness at the group level.

\section{Working definition of intergroup forgiveness}

Based on the outline of definitions above and the claim that reconciliation is the outcome of forgiveness (see McLernon, Cairns, Lewis, \& Hewstone, 2003; Montiel, 2000, 2002; Noor, Brown, \& Prentice, 2008), intergroup forgiveness is defined as follows.

Intergroup forgiveness is a contextualized, dynamic process between the victimized and perpetrating party that involves negotiation and understanding. It is an unfolding and continuous process, in which the perpetrator party provides reasons for the victimized party to consider forgiveness and reconciliation. Importantly, it involves psychological closure for both parties (e.g. a form of 'social healing'), in which a symbolic departure from the past is reached. A shared and consensual interpretation of the conflict between both parties is necessary in order for them to be able to empathize and gain trust with the other side. The process entails acknowledgment of harm done and a public apology that includes significant and coherent actions, and an acceptance by the victimized party that the approach by the perpetrator party is appropriate and genuine, leading to an open dialogue. This dialogue may include sharing historical understandings of the 
conflict. According to research on the collective remembering of conflict (Pennebaker et al., 1997; Páez \& Liu, in press), divergent versus consensual interpretations of the past between formerly conflicting nations is crucial in understanding why societies today are either reluctant or eager to engage in peacemaking efforts to resolve outstanding disputes.

\section{The psychology of intergroup forgiveness}

"Against the backdrop of a world in which hatred and resentment loom larger than amity and recognition, forgiveness appears an oddly idealistic theme to emerge as central to some recent political thinking." (Janover, 2005, p. 222)

Forgiveness between peoples and nations is capturing the attention of scholars across a range of disciplines (Oliner, 2005). The inability of human kind to live peacefully together without perpetrating psychological or physical harm on one another is the reality of the world we live in (Beatty, 1970; Oliner, 2008). Hence, it makes responses and experiences of forgiveness necessary in order to facilitate peaceful coexistence (McCullough, 2008). This thesis' focus is the setting of post WW II and the application of forgiveness in this setting comparing the European and East Asian context. In the following section, I will summarize previous research conducted in this domain and critically evaluate them.

\section{Social Identity Approach and the Contact-Hypothesis}

The steadily increasing psychological research on intergroup forgiveness has been carried out in the post-conflict context of Northern Ireland (e.g. McLernon, Cairns, Hewstone, \& Smith, 2004; Noor, Brown, \& Prentice, 2008; Tam, Hewstone, Cairns, Tausch, Maio, \& Kenworthy, 2007), Chile (Manzi \& Gonzáles, 2007) and in Bosnia and Herzegovina (Čehajić, Brown, \& Castano, 2008). These studies have several points in common: the main theoretical 
framework is provided by social identity theory (SIT; Tajfel \& Turner, 1986) and self-categorization theory (SCT; Turner, 1987, 1999) and/or the application of the contact hypothesis (Allport, 1954). The investigations focused on within-society dynamics (for example Protestants versus Catholics in Northern Ireland; Left-wing versus Right-wing in Chile). The main variables used to predict intergroup forgiveness in these contexts were SIT/SCT driven: group-based emotions, contact, outgroup attitudes, and affective concepts such as empathy, trust and competitive victimhood. Moreover, identity concepts with varying levels of inclusiveness (ingroup, common ingroup identity, human identity) were examined. These studies were either cross-sectional, longitudinal or experimental (mostly scenario-based studies). As intergroup forgiveness research is a rather young field, the key focus has been on understanding how forgiveness relates to other concepts and what drives intergroup forgiveness. The focus on similar research paradigms and underlying mechanisms of intergroup forgiveness has led to some comparable findings. I will summarize the social identity approach in the next section followed by a review of empirical research on intergroup forgiveness.

Social Identity Approach: Social Identity Theory (SIT) and Self-categorization Theory (SCT)

Social identity theory (Tajfel \& Turner, 1979, 1986) and its follow-up selfcategorization theory focusing on the cognitive component of SIT (Turner, 1987; Turner, Hogg, Oakes, Reicher, \& Wetherell, 1987; Turner, 1999) are among the leading theories on intergroup relations. Social identity theory emphasises the social context and its influence on intergroup relations (Hornsey, 2008). The development of SIT was a European response and critique to the American social cognition paradigm, which has an individualistic and asocial interpretation of social psychology (for a review see Hogg \& Williams, 2000). 
SIT distinguishes between personal identities (interpersonal spectrum) and social identities (intergroup spectrum). Personal identity reflects self-definitions in terms of personal attributes, whilst social identity is how people regard themselves in terms of their social category membership (Turner, 1999). Overall, SIT argues that intergroup relations are influenced by an interaction of cognitions (identification with a social category), motivation (being positively distinct from an outgroup and attaining a positive social identity), social comparison, and sociohistorical components (social situations) (Hornsey, 2008).

Social interactions incorporate a shift from the interpersonal spectrum to the intergroup spectrum depending on how individuals see themselves in relation to others (Hornsey, 2008). Furthermore, this shift is a function of an interaction between psychological and social factors (Turner, 1999). Individuals depend on contextually relevant signs to help them to define themselves in relation to a group and others' group membership. Turner (1975, p. 10, cited in Hogg \& Williams, 2000) argues that behaviour between groups can be regarded as "a process of competition for positive identity". As soon as there is a salient "us and them" distinction, it alters the view of how people see themselves and others.

One goal of social identification is to find psychological meaning by identifying a certain group category through a social comparison process. Only through comparison with other groups does a particular group membership become psychologically meaningful (Hogg \& Williams, 2000). The ultimate aim of this comparison process is to attain a positive social identity and to be positively distinct from the relevant outgroup(s). The strength of the identification with a particular group determines the drive to gain a positive social identity and a sense of belonging. This motivation can result in ingroup favouring behaviours resulting in an ingroup bias, and in the process of social comparisons may result in outgroup 
derogation. The argument that ingroup favouritism is a sign of outgroup derogation, however, is disputed (see Brewer, 1979, 1999). Brewer (1999) argued that prejudice and discrimination are motivated by ingroup favouring behaviours rather than direct aggressions against outgroup members. Therefore, SIT is better in explaining ingroup favouritism than outgroup hostilities (Hornsey, 2008). According to Turner (1999), SIT proposed a clear line between individual and group behaviour, but it does not answer the question how social identities become salient.

Turner and colleagues (1987) moved beyond the intergroup focus of the SIT framework to elaborate on intragroup processes. They tried to address unanswered questions regarding the process of social identification and the interrelationship between interpersonal and intergroup behaviour. Although SIT and SCT overlap in terms of their theoretical underpinnings, these two theories have different foci (Hornsey, 2008). SCT deals with the role of the categorization process. Turner et al. (1987) and Turner (1999) examined the interrelationships between interpersonal and intergroup behaviours stemming from an underlying distinction between personal and social identity. They argued that identity operates at different levels of inclusiveness rather than as a dynamic shift between bipolar ends (Hornsey, 2008). They distinguished three levels: a superordinate level (self as a human being), an intermediate level (social identity), and a subordinate level (personal identity). The salience of one aspect of a person's identity is in some situations higher whereas the other two are lower. Therefore, in SCT identity is a flexible construct and is not static.

Social categorization arises when social categories are perceived to represent social reality and when these categories deliver means for differentiation from an outgroup (comparative fit). SCT argues that this process is dynamic 
because it varies depending on the context. This variation occurs according to the accessibility and fit of a category. A social category may fit well if the group behaviour and membership are consistent with stereotypical expectation or a group prototype (normative fit; see Hornsey, 2008). The salience of a social category also depends on its accessibility in a situation. For instance, it may be primed because of frequent salience or due to motivation to activate a particular category (Hornsey, 2008).

Hogg and Williams (2000) propose that the salience of social identities is driven by an interaction of five elements. These are individual motives self-esteem and uncertainty reduction, the accessibility of the social category dependent on the context and on memory, how well a social category helps to differentiate between people, and how well a social category can explain others' behaviour. According to Turner (1999) when a shared identity is salient, people tend to perceive themselves and other ingroup members as interchangeable exemplars of the same social group. This is termed prototype-based depersonalisation. Turner (1999) deems this process as the core psychological process of group behaviour.

What started as an ambitious response to mainstream American social psychology developed into one of the most influential, widely accepted and most applied theories to understand intergroup phenomena. However, the social identity approach also received criticism. SIT has been criticized for not being falsifiable, which means that the theory has become overextended: almost any outcome is explainable within its overarching framework (Hogg \& Williams, 2000). Hogg and Williams pointed out that this is a weakness rather than strength.

Farr (1996, cited in Hornsey, 2008) sees the claim of social identity theory as being nonreductionist and collective as contestable, as he argues that it represents the reductionist and individualist working shoulder to shoulder with 
mainstream social cognition. Furthermore, Brewer $(1979,1999)$ questioned the specific presumption that ingroup favouritism and outgroup derogation are reciprocally related. The original SIT proposition that social identity (collective self) and individual self are cognitively independent has also been contested (Hogg \& Williams, 2000; Markus \& Kitayama, 1991). Duveen (2001) criticized SIT and SCT for focusing too much on the outcome of the identification process and too little on what an identity entails in each social situation. Overall, there are reasons to question the scope of identity for investigating intergroup forgiveness, since intergroup forgiveness is tied to a conflict that has a history and has several groups as agents involved in a conflict, which also have a history. The application of social identity to understand intergroup forgiveness runs the risk of being reductionist, since intergroup forgiveness is a very complex process and cannot be solely understood and reduced to issues over identity.

\section{Contact Hypothesis}

Allport (1954) originally formulated the contact hypothesis, proposing that intergroup relations can be improved through frequent positive interactions between members of different groups. Generally, frequent contact with outgroup members can alter stereotypes about the outgroup, increase perceptions of heterogeneity of the outgroup, and reduce prejudices (e.g. Rothbart \& John, 1985). Furthermore, positive contact experiences with representative outgroup members can also reduce anxiety towards the outgroup as a whole (Hewstone, Cairns, Voci, Hamberger, \& Niens, 2006).

In order to be effective, interpersonal contact between rival groups should take place under four optimal conditions: equality of social status, purposeful cooperative activity, common goals and social and institutional support (Pettigrew, 1998). The most widely accepted perspective has focused on changes in 
categorization. Namely, decategorization (Brewer, Manzi, \& Shaw, 1993) and recategorization of group categories can explain the effectiveness of intergroup contact to reduce prejudice (Gaertner \& Dovido, 2000). Decategorization means to perceive oneself as a separate individual and not as a member of a group. Hence, decategorization loosens group boundaries; interactions between individuals take centre stage and the salience of distinctions between groups become redundant. Studies have provided empirical evidence for the existence of this effect (for an overview see Gaertner \& Dovido, 2000). Emphasis on a common identity or recategorization of group categories (Common Ingroup Identity Model, CIIM; Gaertner \& Dovidio, 2000) may lead to the transformation from two (rival) groups into one inclusive group, and can alter perceptions of outgroup members (Gaertner \& Dovidio, 2000). Overall, the contact hypothesis has been referred to as "one of psychology's most effective strategies for improving intergroup relations", (Dovidio, Gaertner, \& Kawakami, 2003, p. 5).

The contact hypothesis has also received criticism doubting the applicability of the optimal contact strategy, as it seems detached from social reality (e.g. Dixon, Durrheim, \& Tredoux, 2005). The emphasis on positive factors in the contact situation rather than factors that may inhibit the reduction of prejudice and intergroup anxiety has been questioned (Hewstone et al., 2006), but remains under-researched (see Dovido et al., 2003; Pettigrew \& Tropp, 2006).

The criticism is justified when it comes to the aftermath of large-scale conflicts within which members of societies may, but more likely may not, have contact with other members of the societies (e.g. the aftermath of World War II in East Asia). The initial proposition of the contact hypothesis was about the reduction of prejudice by increasing of the quantity and quality of contact between 
members of different groups. Most of the later propositions of the contact hypothesis are based on social identity theory and self-categorization theory.

A recent meta-analysis carried out by Pettigrew and Tropp (2006) gathered empirical evidence about the existence of a robust link between contact and the reduction of intergroup contact. This robust link goes beyond contact between racial or ethnic groups and is empirically independent from Allport's proposed optimal conditions. Pettigrew and Tropp (2006) recommend to focus more on the inhibitors of intergroup contact and to conduct longitudinal studies in order to establish causal factors that lead to the persistence of positive effects of intergroup contact.

\section{Empirical research on intergroup forgiveness}

The next section reviews empirical research on intergroup forgiveness with the aim to summarize the main findings in studies that have applied the aforementioned theoretical frameworks. I will close the review by pointing to gaps in the literature and suggest an alternative theoretical framework for the investigation of intergroup forgiveness.

\section{Identity}

Research focusing on the identity aspects of intergroup focus process aimed to provide insight into the role and future of intergroup forgiveness in real post conflict settings using survey studies. Identification with one's ingroup (SIT; Tajfel \& Turner, 1979; Turner, 1999), or with a common identity (CIIM; Gaertner \& Dovido, 2000) was examined as either a mediating variable (e.g. Noor et al., 2008), a moderating variable (Hewstone et al., 2004) or a direct predictor (e.g. Cairns, Tam, Hewstone, \& Niens, 2004; Noor, Brown, Gonzales, Manzi, \& Lewis, 2008) of intergroup forgiveness. 
Noor and colleagues (2008) argued that intergroup forgiveness relates to identity concerns. Drawing from SIT, Noor and colleagues further argued that this distinguishes interpersonal from intergroup forgiveness. Moreover, they proposed that the strength of identification is an underlying force that drives the relationships between the independent variable (e.g. guilt) and intergroup forgiveness. In most studies, ingroup identification was negatively associated with intergroup forgiveness. When tested as either a mediator or moderator, ingroup identification was a significant contributor to reported levels of intergroup forgiveness. A common identity or superordinate group category was positively associated with forgiveness in Northern Ireland, Chile (Noor et al., 2008), and (to a marginal degree) in Bosnia and Herzegovina (Čehajić, Brown, \& Castano, 2008). Hence, common identity seems to work differently in some samples, as the moderating effect was only significant among the Catholic sample in Northern Ireland (Noor et al., 2008).

The moderating role of ingroup identification has been tested in experimental designs, using scenarios describing an act of paramilitary violence with various intentions and motives to assess the effect of ingroup identification on intergroup forgiveness (Cairns et al., 2004). These studies found that ingroup identification drives a bias towards intergroup forgiveness over and above whether the harm done was perceived to be intentional. High identifiers showed a strong ingroup favouring bias in which they showed more forgiveness when the perpetrator was an ingroup member. There was no differentiation for low identifiers between ingroup or outgroup perpetrators in terms of levels of forgiveness. This effect occurred regardless of the perceived intentions and motives of the act. 
However, as I will point out in the section on research gaps, although this research clearly pointed out the importance of investigating identity and the consequences of identification, it overlooks the relevance of the historical context as an influencing factor. Another reason for concern is that within-group dynamics possibly do not apply to between-group dynamics, such as relationships between nations involved in WW II. For example, little is known about how the various ways that Germany and Japan have dealt with their perpetrator role in WW II has affected or still affecting the relationship between nations rather than groups within a nation or region. In other words, the representation of the historical past may have a substantial impact on intergroup forgiveness between nations.

Wohl and Reeder's (2004) series of experiments with hypothetical scenarios showed that ingroup members tolerated aggressive behaviour by ingroup members, if the behaviour was beneficial for the group. The act was judged as immoral, but the ingroup member was forgiven. On the other hand, outgroup members were judged harshly when the outgroup member undertook similar aggressive acts. Outgroup members were judged as less moral for the same offence and were not forgiven.

Similarly, Wohl and Branscombe (2005) tested the mediating role of a human-level categorization and social identity categorization in group-level reactions to the Holocaust. Contemporary Jewish people were asked how forgiving they were towards today's Germans. When Germans were seen as fellow humans rather than Germans, contemporary Jewish participants were more forgiving.

In another series of scenario-based studies in Lebanon, which included Catholics, Maronites, and Orthodox Christians, various vignettes were presented with varied scenarios: cancellation of consequences, intent to harm, religious and social proximity, and apologies (Azar, Mullet, \& Vinsonneau, 1999). The results 
showed slightly different patterns to the aforementioned studies. The only social factors that affected the propensity to forgive were the level of education. Better educated people expressed more willingness to forgive, regardless of community membership. Gender and age did not influence the findings. Therefore, the community membership was not important and participants expressed equivalent levels of forgiveness. A replication by Azar and Mullet (2001, cited in Azar \& Mullet, 2002) of the same experiment, but with different communities in Lebanon (Druzes, Shiites, and Sunnis), confirmed previous findings: equivalent levels of forgiveness were expressed independent of the community membership of the offender.

Therefore, it could be argued that the ingroup-outgroup differentiation is governed by the historical and social context rather than the mere social categorization process. One can speculate that depending on the methods (survey or an experiment), group membership is sometimes highly salient and sometimes not in previous research. A key question is why identity was driving the outcome so strongly in some settings, whilst in other settings it did not seem to impact. This inconsistent finding deserves further attention. One explanation could be the underlying historical context in given conflict (and interpretation of that context) can have different effects on how group boundaries are created and maintained (Breakwell, 2001; Hilton \& Liu, 2008; Liu \& Hilton, 2005). Considering - as Hartwell (2005) and Staub (2000) point out - that the roots of many current conflicts stem from past conflicts between nations or groups, the historical context is an essential force that should not be ignored.

\section{Contact}

In divided societies like Northern Ireland or Bosnia and Herzegovina, it seems appropriate to test the impact of cross-community contacts on intergroup 
forgiveness. Overall, the findings indicated that cross-community contacts have a positive impact on intergroup forgiveness, confirming previous research on the positive effects of intergroup contact. These studies focused on either the effects of contact in integrated or mixed education in schools where Catholic and Protestant pupils share the same class-rooms in Northern Ireland (McGlynn, Niens, Cairns, \& Hewstone, 2004); or the assessment of a self-reported measure of contact with the outgroup (Cairns et al., 2005; Čehajić, et al., 2008; Moeschbeger et al., 2005; Tam et al., 2007). The direct effects of contact were usually mediated by other variables, such as ingroup identification or infrahumanization (i.e. seeing the outgroup as more human); or group-based emotions like anger, empathy, outgroup attitudes.

Tam and colleagues demonstrated (2004; cited in Cairns, 2005) that contact resulted in lower infrahumanization and promoted forgiveness. This mediation was stronger than the mediating effects of anxiety, empathy and respect variables. Čehajić and colleagues (2008) showed that the direct effect of intergroup contact and intergroup forgiveness was mediated by empathy, trust and outgroup heterogeneity. The positive impact of intergroup contact on trust, empathy and outgroup heterogeneity had an indirect positive effect on intergroup forgiveness. In Moeschberger, Dixon, Niens, and Cairns (2005) study, contact mediated the effect between empathy and forgiveness.

In sum, contact contributed to promote intergroup forgiveness in divided societies like Northern Ireland and Bosnia and Herzegovina. However, one may point cautiously to the fact that the focus was on within-society dynamics where (structured) contact (e.g. integrated schools) between conflicting groups may be a practical solution. This is not the case in conflicts between societies. Furthermore, there were some inconsistencies in these findings. For example, in Hewstone and colleagues' (2004) study with a large general population sample, contact was a 
positive contributor for the prediction of forgiveness for Catholics, but not for Protestants. Likewise, there were significant differences between Catholics and Protestant on many of the assessed variables (e. g. Catholics scored higher on forgiveness and trust than Protestants), whereas in other studies there were no differences between the two groups and samples were collapsed for more powerful statistical analyses (e.g. Noor et al., 2008; Hewstone et al., 2004). These studies fall short on explaining why the processes of forgiveness in Catholic and Protestant samples were different. The context of these conflict settings were explained and described, but the historical background was not considered as a source of further explanations or predictions.

The following section reviews previous research on group-based emotions, such as collective guilt, collective shame and group-based anger and their effects on intergroup forgiveness.

\section{Collective guilt and collective guilt assignment}

In Northern Ireland the boundaries between victims and perpetrators are blurry as these roles are not clear and intertwined. During this conflict, victims became perpetrators themselves and vice versa, resulting in competitions over victimhood, which in turn may lead to inhibit the promotion of forgiveness and foster mutual guilt assignment over the past harm done (Noor et al., 2008; Noor et al., 2008). Collective guilt can be experienced when people categorize themselves into a group that has committed harm against another group (Branscombe \& Doosje, 2004; Wohl \& Branscombe, 2005). Collective guilt is a moral emotion based on an appraisal of the responsibility of one's ingroup for past harm done that motivates reparation (Iyer, Schmader, \& Lickel, 2007). It has been identified as a positive antecedent for intergroup forgiveness in the Northern Ireland context (e.g. Hewstone et al., 2004). As already discussed, feelings of guilt towards the other 
group enhances willingness to forgive the group who suffered due to one's ingroup (e.g. Hewstone et al., 2004). Manzi and Gonzáles (2007) showed that collective guilt had an important role in predicting forgiveness and reparation attitudes the context of Chile (Left wing vs. Right wing). Feelings of collective guilt towards an outgroup are less likely to occur when a person has high experiences of victimization (Hewstone et al., 2004).

It is suggested that collective guilt functions to allow people to positively distinguish themselves from former perpetrators. Any act that would induce some sort of guilt feelings are thus rejected by people who identify highly with former perpetrating group, as opposed to those who identify not as strongly (Branscombe \& Doosje, 2004; Montada, Schmitt, \& Dalbert, 1986; Schmitt, Behner, Montada, Müller, \& Müller-Fohrbrodt, 2000). Indeed, Hewstone and colleagues (2004) demonstrated that low ingroup identifiers felt more collectively guilty than high identifiers. However, even among theorists, the idea that a person can be guilty for something they have not done themselves is controversial.

Collective guilt is assigned when people perceive that another group has violated moral standards (Wohl \& Branscombe, 2005). Being a member of a historically victimized society can lead to the expectation that former perpetrators should feel sorry and try to make up for what their ancestors have done in the past. If the former perpetrators or representatives of the former perpetrating society fail to fulfil these expectations, the former victims may feel resentful and therefore would assign more guilt. Furthermore, if collective guilt is assigned to a great extent, it is possible that there is no closure with the past and no grounds to depart from the past. The assignment of guilt depends on the level of social categorization of the outgroup. This was demonstrated by the perception of Germans as fellow 
humans resulting in less guilt assignment by Jews than seeing the Germans as Germans (Wohl \& Branscombe, 2005).

\section{Collective shame}

The appraisal of responsibility of harmful actions is also a basis for collective shame. Shame elicits different attributions for the harm doing than guilt and anger feelings (Brown et al., 2008; Iyer et al., 2007). While guilt focuses on the action and the perception of control over the action, shame focuses on factors that are not controllable, such as the characteristics of the group being exposed as weak or even disgusting (Branscombe \& Doosje, 2004). Lickel, Miller, Stenstrom, Denson, and Schmader (2006) argued that guilt and shame have unique behavioural consequences: guilt educes more approach behaviour, like the motivation to repair the damage made, whereas shame elicits withdrawal from the situation. In three cross-sectional studies with non-indigenous Chileans in the context of the historical maltreatment of the Mapuche, Brown and colleagues (2008) revealed that collective guilt and shame predicted reparation attitudes. Furthermore, they provided evidence that collective guilt had longitudinal effects for the increase in reparation attitudes for low-shame people. Collective shame only seemed to have short-term effects on reparation attitudes, and was mediated by the desire to improve the ingroup's reputation.

\section{Group-based anger}

Intergroup relations often entail strong emotions. Mackie and Smith (2002)

criticized social psychologists for tending to overemphasize cognitive processes in intergroup relations and failing to acknowledge the role of group-based emotions. Intense emotions such as anger can contribute more to predicting intergroup behaviour than cognition-based evaluations. In the context of intergroup conflict, 
group-based anger is maintained through intra-group memories of the conflict, long after the violence and hostilities have stopped (Manzi \& Gonzáles, 2007; Bar-Tal, 2000). Furthermore, anger is related to a reluctance to forgive (Baumeister, Exline, \& Sommer, 1998) and seems to drive actions against people associated with the former perpetrator group. In previous research, anger was strongly negatively associated with forgiveness and willingness to repair relationships (see Manzi et al., 2007; Tam et al., 2007).

Concluding remarks about SIT/SCT based intergroup forgiveness research

In sum, research applying the SIT and SCT paradigm found strong evidence that post-conflict intergroup forgiveness is influenced by identification and contact, and is supported by the development of positive attitudes. Although Worthington (2005) has pointed out that interpretations of events probably influence the process of forgiveness, previous research has not taken the historical context and the consequences of different historical accounts into consideration. Furthermore, little is known about how forgiveness processes work between nations rather than within a nation or region. Moreover, it is also disputable how contact can help resolve conflict for groups that do not usually have frequent contact or do not have the opportunity to get in contact under optimal conditions. Another point of concern is that most research has been conducted by Western psychologists with a focus of individualistic paradigms (e.g. desire to obtain a positive social identity), which may not apply in every non-Western contexts.

The following section considers the research that has been conducted in cross-cultural settings and that had an applied focus. 


\section{Cross-cultural and applied research}

Cross-cultural research on intergroup forgiveness is virtually non-existent. The handful of studies that have been conducted are devoted to examining the applicability of this concept in settings after gross human rights violations (Kadima Kadiangandu \& Mullet, 2007; Neto, Pinto, \& Mullet, 2007a). The research showed that participants perceived intergroup forgiveness to be an important concept and favoured implementations in political processes similar to the Truth and Reconciliation Commissions in South Africa. Furthermore, one study by Noor and colleagues (2008) empirically demonstrated that similar mechanisms underlie forgiveness processes in Northern Ireland and Chile with varying strengths. However, this was not the focus of their study.

There are to date only a few studies that consider indigenous conceptualizations of intergroup forgiveness (Mellor, Bretherton, \& Firth, 2007; Rata, Liu, \& Hanke, 2008). These studies indicated that in unresolved disputes with unbalanced relationships, as it is the case in the Aborigines-White Australians and Maori-Pakeha relations, granting forgiveness is associated with potential costs that in turn threaten rapprochement between groups.

Staub's $(2000,2006)$ research had an applied focus and was devoted to understanding the origins of genocide and mass killings, its prevention, and the possibilities of healing and reconciliation in the aftermath of such human tragedies. One of his focuses has been the Rwandan genocide in which during a 3-month period in 1994, 800,000 people were killed. In a number of projects in collaboration with local organizations in Rwanda, Staub, Pearlman, Gubin and Hagengimana (2005) designed a theory-based intervention to foster healing among victims and perpetrators. The intervention was based on five key elements (Staub et al., 2005, p. 304-306): (1) understanding the roots of the conflict and genocide, (2) 
understanding the effects of trauma, victimization and paths to healing, (3) understanding of basic psychological needs, (4) sharing painful experiences in an empathic context and (5) vicarious traumatisation. In sum, experimental evaluations of this intervention showed that (a) the intervention reduced trauma symptoms and increased a positive orientation towards outgroup members; and (b) observations implied the importance to understand the origins of violence as this fostered empathy.

In the following section, I evaluate previous research and point to gaps in the literature.

\section{Research gaps and critical evaluation of previous research}

The reviewed research predominantly conducted by Western psychologists demonstrated the relevance of ingroup identification, intergroup contact, and group-based emotions to the intergroup forgiveness process. Intergroup forgiveness seems to work as a precursor for sustainable reconciliation and to benefit both those who forgive and who are forgiven. This research has mostly been carried out within one society in the Western hemisphere (e.g. Northern Ireland). Furthermore, applied research yielding interventions in the aftermath of human rights violations has been conducted in the context of the Rwandan genocide. In Lebanon, East Timor and Congo research in a particular conflict situation has been undertaken to understand the meaning of forgiveness and its utility in interventions for peace. The East Asian context is continuously neglected, but may add to a more holistic understanding of the complex process of intergroup forgiveness. Some scholars have argued that East Asian societies may carry "the burden of past conflict" post WW II more heavily than European societies, and therefore are important to include in intergroup research regarding this conflict (Buruma, 1994; Hein \& Selden, 2000; Oliner, 2008). The historical, cultural and political context is critical 
for both a practical and deeper theoretical understanding of intergroup forgiveness (Montiel, 2000, 2002).

Cairns and Roe (2003) noted that the intergroup conflict literature often neglects the historical context of real life conflicts. They refer to memory issues that perpetuate conflicts. The studies conducted within the SIT and SCT frameworks showed that the role of identity and competing identities (e.g. victimhood) significantly influenced intergroup forgiveness. However, Cairns and colleague pointed out that identities are often constructed based on the historical context. However, the content of identities has been largely neglected in this research tradition, as it solely focuses on the consequences of the identification process (Duveen, 2001). Therefore, social identities in that sense are too often "empty forms" and have "become a vacuous notion, a transparent glass enclosing nothing" (Duveen, 2001, p. 267).

Liu and Hilton (2005) offered a theoretical solution to this dilemma through integrating social representations of history and the relevance of identity. The historical event World War II (WW II) has universal meaning and is a predominant representation of global history (Liu \& Hilton, 2005). At the same time, it produces different content based on different group's representations of history, which influences group processes and defines how groups relate to one another. For example, from the same set of events and figures represented in WW II, Germany and the USA may draw different conclusions for future challenges. The USA sees itself as a protector of democracy and justifies present military interventions, through pointing to WW II and the role of the USA in ending Nazism. Germany as a former enemy and present ally may produce different perspectives in public. German leaders can both remind the public of the horrors of war and therefore seek to denounce military interventions. On the other hand, public reminders of 
Germany's moral responsibility to protect Jews in Israel can motivate the support of military interventions (see Liu \& Hilton, 2005). In the following section, I will briefly summarize the Social Representations Theory (SRT) and its application to history and identity (see Hilton \& Liu, 2008; Liu \& Atsumi, 2008; Liu \& Hilton, 2005, Liu \& László, 2007).

\section{Social Representations Theory (SRT)}

Social Representations Theory (SRT) emerged similar to SIT as a European response to the American mainstream social psychological paradigm almost 50 years ago, and has been outlined by Moscovici (1984). In general, social representations are shared conceptions about the world structured and processed through the implementation of new knowledge shared in societies. László (2003) argued that SRT "investigates the process and content of representations in relation to social dynamics" (p. 182). The main objective of SRT is to link the society to the individual and the transformation of knowledge across societies. This already poses a problem since psychology as a discipline focuses on the individual level of analysis (Liu \& László, 2007). SRT was formulated to bridge the divide between social and psychological phenomena, with emphasis on its novel and dynamic nature (László \& Wagner, 2003).

Moscovici (1988) argued that "our past experiences and ideas are not dead experiences or dead ideas, but continue to be active, to change and to infiltrate our present experience and ideas. In many respects, the past is more real than the present" (p. 10). Furthermore, he suggested that social representations change and influence individual behaviour in a collective. Moscovici distinguished two mechanisms of thought processes, which are relevant in making the unfamiliar familiar, or in other words, convert the new and unknown into the known. These mechanisms are called anchoring and objectification. Anchoring is setting alien or 
new ideas in a familiar context by classification and naming, which assists us to imagine and represent something. Objectification is transforming something abstract into something that is concrete by reproducing "a concept in an image" (Moscovici, 1984, p. 38) that has a clear meaning in reality and becomes physical and accessible. Furthermore, Moscovici argued that social representations are dependent on memory. Anchoring and objectification are means to handle memory and to manage cognitions.

Three types of social representations can be identified. Namely, these are hegemonic (representations shared by all members of a society), emancipated (development and circulation of shared knowledge and ideas within subgroups, alternative versions for each sub group) and polemical representations (generated due to social conflicts within societies, not shared, generated by opposing relationships between groups) (Breakwell, 2001; Brewer, 2001; Moscovici, 1984). Brewer (2001) referred to social representations as the "'glue' of social life" (p. 308) and suggested that shared meanings determine group boundaries, since social representations provide content for social groups.

There are three means to communicate, transmit and maintain social representations (Staerklé, 2006). Namely, this occurs through propaganda, propagation, and diffusion. Propaganda is about promoting a particular institution's or group's representations (these can be associated with stereotypes); propagations are attitudes where social representations of a group defend their point of view, but also accept different views; and diffusions are widely accepted opinions with little or no resistance to their validity (Moscovici \& Marková, 2000; Staerklé, 2006). Therefore, hegemonic representations' means of transmission are diffusions; emancipated representations' means are propagation; and polemical representations' means of transmission are propaganda. 
One example of the transmission of knowledge about the atrocities committed by Japan during WW II (e.g. Comfort Women Issue and the Nanjing Massacre) would be how the Japanese "Association for liberal views of history" communicate their view. This representation can be regarded as propaganda as their view generates negative stereotypes of China in claiming that Chinese views on this matter are false and that Chinese evidence as well as Chinese testimonies have been fabricated (e.g. Takemoto \& Ohara, 2000). An example of diffusion would be how knowledge about the Holocaust has been communicated via textbooks in Germany. Perspectives on the Holocaust are widely accepted there and not publicly contested as it is the case in Japan (Hein \& Selden, 2000; Oliner, 2008).

Similarly, Voelklein and Howarth (2005) discussed the role of representations in constructing symbolic boundaries that define social identities. They proposed that the essence of representations as being social stems from their creation by collectives and validation through social processes. Additionally, they argued that the content of social representations is affected by the historical and cultural context. Therefore, there is the possibility that social representations precede social identities (see Brewer, 2001; Duveen, 2001; Voelklein \& Howarth, 2005). However, there is also considerable speculation that social identities come first (Breakwell, 2001) or that there is mutual exchange between the two (Brewer, 2001). Brewer (2001) acknowledged that there is no conclusive argument for a priority of social identities over social representations and vice versa, since there are valid cases for both positions. Recently, Wagner and Hayes (2005) argued for a hermeneutical circle of meaning that connects identity and social representations.

According to Raudsepp (2005) social representations are group-level phenomena and are an expression of a social process between members of a social 
group rather than aggregates of individual minds. Furthermore, she argued that social representations function in different forms according to the level. At the individual level, social representations provide content; at the interpersonal and intergroup level, they develop through the process of communication or interaction; at the cultural and macro-social level, social representations are objectified in cultural or societal products. An interesting aspect of social representations is the notion of collective emotions fostering actions (Moscovici, 1998). This is in line with group-based emotions theorists promoting the importance of emotions in eliciting action-tendencies rather than cognition-based evaluations (Mackie \& Smith, 2002).

Raudsepp (2005) raised another interesting point, which I would like to use to transfer to the more specific focus of social representations of history. The main criticism regarding SRT is directed at theoretical problems. Since SRT is mainly theoretical itself, this is understandable. However, Raudsepp (2005) addressed the applicability of SRT to real life social problems. The point is that different social representations may exist for the same social object or event. These differences can evoke tensions and conflicts, which is not negative per se as it adds to the development of belief systems (Moscovici \& Marková, 1998, cited in Raudsepp, 2005). However, the downside is that it may induce conflicts at the symbolic level, if these representations are polemical rather than hegemonic. Many conflicts nowadays are rooted in the past and are symbolic in their nature. Raudsepp argued that these are "mediated and regulated by certain social representations and social identities related to them" (p. 465). With special reference to disputes over the right account of historical events during WW II, she concluded that social representations need to be transformed to overcome conflicting group interests where a dialogue and the necessity of re-elaborations of common beliefs. This 
conclusion comes very close to practices of textbook commissions between Germany, France and Poland regarding WW II textbook accounts aiming for a shared understanding of the past (Ruchniewiecz, 2005; Viedt, 1993) and Staub's and colleagues' (2005) intervention component of understanding the roots of the conflict. The following section describes social representations of history and their relevance for intergroup forgiveness.

Social representations of history and intergroup forgiveness

"...too much memory or not enough; too much enshrinement of victimhood or insufficient memorializing of victims and survivors; too much past or too little acknowledgment of the past's staging of the present ..." (Minow, 1998, p. 2)

Social representations of history are relevant for group cohesiveness and continuity as "history is the story of the making of an in-group" (Liu, Wilson, McClure, \& Higgins, 1999, p. 1023). The historical past of peoples and how it is communicated within and between social units is matter of social representation processes (László, 2003). Even today, many people still feel the consequences of the deadliest conflict in human history. For example, World War II (WW II) with all its connotations has become a crucial part of German history and the positioning of German identity today (Buruma, 1994; Hauss, 2003; Oliner, 2008). Germany’s past war crimes (especially the Holocaust) are symbols of extreme atrocities and frequently used as a reference point when other crimes against humanity are committed. However, it is generally accepted by Germany's neighbours, especially in Western Europe, that Germany has expressed genuine contrition for her war crimes, and moreover that the Germany of today has broken with any sense of political continuity with Hitler's Germany. 
Atrocities committed during WW II and the Sino-Japanese War in East Asia by Japan are less familiar to Westerners (Hein \& Selden, 2000). However, the atrocities are salient, play an important role on the victimized side and have an unresolved legacy. That is, Japan's neighbours are less confident of Japan's genuine contrition for her misdeeds in WW II, less confident that Japan has fully condemned past harm done and that a decisive break with the past has been made. As a consequence, there are regular disputes over the representation of WW II in East Asia (Liu \& Atsumi, 2008), including controversies over textbook accounts of WW II officially sanctioned by the Japanese government, and visits to the Yasukuni shrine (where Class A war criminals are interred) by the Japanese Prime Minister. Hence, the relationship between Japan and her East Asian neighbours (especially China and Korea) still suffers from lack of closure to narrative conflicts stemming from WW II (Hein \& Selden, 2000; Liu \& Atsumi, 2008; Oliner, 2008; Yamazaki, 2006). Therefore, the disputed representations of the historical past have the form of polemical representations that cause conflicts on a symbolic level (Breakwell, 2001; Brewer, 2001; Moscovici, 1984).

Even more than a half century after the end of WW II, differences in the meaning of WW II impacts on international relations in Germany and East Asia. Therefore, differences in the content of the collective remembering of historical conflict produce different social representations. This may lead to differences in the propensity to forgive for WW II atrocities between the European and East Asian contexts.

Liu and Hilton (2005) argue that social representations of history give a people anchors that help define ethnic and national identities, provide guidance on how to relate to other groups, and to construct and maintain a distinct political culture (Sibley \& Liu, 2007; Liu \& Sibley, in press). Social representations of 
history provide a useful theoretical framework where the positioning of peoples around the world due to the social context may vary (e.g. the burden of WW II for Germany and Japan in relation to their neighbours). WW II is widely regarded as the most important event in world history (Liu \& Hilton, 2005; Liu \& Sibley, in press). Critical historical events and figures furnish raw materials that political elites can use to shape into symbols of a nation's origin, its current mission and justification for current actions. Representations of history can thus define privileges and responsibilities for groups, and legitimize social and political arrangements, nationally and internationally. Furthermore, a group's representation of history offers explanation as to why countries may respond differently to similar challenges (Hilton \& Liu, 2008).

History is part of the societal and inter-societal context of social relations between groups. That is, when Chinese and Japanese interact, they do so within the social context of an objective situation that is to varying extents infused by the collective remembering of historical experiences between these two groups (see Liu, Yamagishi, Wang, Schug, Lin, Huang, \& Yu, 2009; Takahashi, Yamagishi, Liu, Wang, Lin, \& Yu, 2008). A series of experimental studies using dyadic game theory to study social dilemmas over the internet (Liu et al., 2009; Takahashi et al., 2008) found that that outstanding historical tensions between Japan and China shaped patterns of ingroup favouritism in trusting behaviour during controlled social exchange involving real money. When the two groups interacted, mainland Chinese were more ingroup favouring and more trusting overall than Japanese. The best predictor for ingroup trust were perceptions of historical anger against the outgroup; whereas a different pattern was found between Japan and Taiwan, and a third pattern between China and Taiwan (who have separate matters of conflict between them). This suggests that the social dilemma of trust is indirectly 
influenced by the social context provided by the history of international relations. The collective remembering and social representations of WW II can therefore indirectly influence social context by infusing everyday social interactions with implicit forms of (cultural) meaning that shape and guide behaviour (e.g. Hong \& Chiu, 2001; Hong, Morris, Chiu \& Martínez, 2000; Wertsch, 2002).

History further provides raw materials to refine public discourses that provide content through explicitly guiding people in their behaviours and their opinions (e.g. Marková, 2008; Reicher \& Hopkins, 2001; Sibley, Liu, Duckitt, \& Khan, 2008). For example, Sibley et al. (2008) showed in three studies that historical narratives can furnish ideologies that negate or recognize historical claims for reparation by indigenous peoples for past injustices in New Zealand. They provided empirical evidence that history is an important symbolic resource that can be used to mobilize support for public policies, and to justify political views and actions.

In sum, there are several arguments to support the assumption that differences in how the occurrence and consequences of WW II is interpreted may lead to substantial differences in intergroup forgiveness. Historians have already recounted that the handling of the past in Germany and Japan is qualitatively different and has led to different consequences in interrelations between former perpetrator and victim (e.g. Conrad, 2003; Olick \& Levy, 1997; Shimazu, 2003; von Borries, 2003).

The public confrontation with the past has varied largely between these two contexts (e.g. Oliner, 2008). The confrontation with and the general burden of the past are a vivid part of German identity (Buruma, 1994). In contrast, Japan's postwar representations of the past are more focused on the victim role of Japan allowing "Japanese to shy away from the unpleasant truth that they were also 
aggressors, whose victims in East Asia and elsewhere still demand an apology" (Shimazu, 2003, p. 116). There may be implications in the form of a reluctance to forgive by people who identify that their nation has been victimized by Japan.

However, there are polemical representations present within Japan. The opinions that are communicated outside Japan do not necessarily represent Japan as a whole (Ogawa, 2000). Although there is considerable international debate about some of the textbooks that have been endorsed by the Japanese Ministry of Education that downplay Japan's atrocities during WW II, there is also an opposition present that does not approve of these textbooks (e.g. mostly teachers or grassroots movements, Ogawa, 2000). There is an ongoing cultural and political struggle within Japan due to the textbook controversies and Japan's dealing with the past in general (Nozaki, 2003). This struggle within Japan can have consequences on Japanese opinions about compensation and guilt feelings today.

\section{Conclusion}

There are several conclusions that can be drawn from this literature review. Previous research treated forgiveness between groups as relatively independent from its historical context. There has been a high emphasis on consequences of identification processes, but less or no emphasis on the cultural and historical context, which forms a particular content (e.g. for group identity) that may be more helpful in predicting consequences. Therefore, main concerns can be narrowed down to the elaboration of the outcome of the social identification process that leads to ingroup bias in form of more forgiving attitudes toward ingroup members and more harsh judgment of outgroup members. Furthermore, group-based emotions such as guilt and shame have been found to be strong associates of forgiveness. Although all research has been embedded within a specific conflict 
setting, most studies do not draw on how the historical context shapes the identification or forgiveness process.

Moreover, the majority of research has been conducted in mono-cultural settings driven by mostly Western scholars coming from traditional social identity and self-categorization theory. The examinations' foci were on two (religious or political) groups that have been either a victim or perpetrator at some stage and who live within the same geographical boundaries. In these within-society contexts, it seems adequate to apply the contact hypothesis to counter prejudices and stereotypes under optimal conditions. However, this process may not function between societies in which contact is very difficult to establish. Furthermore, how can these assumptions work when atrocities are in the past and "closed" (like the Holocaust)? There are reasons to consider the historical context and how history is interpreted, since we will only find one piece of the complex intergroup forgiveness puzzle if the focus remains solely on identity. Hence, social representations of history offer a complementary approach to accommodate social identity processes and consider the historical context in intergroup forgiveness research.

To address the first research gap, I will employ a cross-national metaanalytical procedure in Study 1 (Chapter 3). Context in previous research was usually represented through varying the situation or the severity of the harm done experimentally. These contextual variables are very proximal to the harm done. This does not do justice to the complexity of the process. It is possible that the social and the historical context within which individuals are nested affects even variations in interpersonal forgiveness. A meta-analysis can shed light on how variations in the context affect variations in forgiveness. Hence, Study 1 aimed to 
explore the impact of contextual variables and the possibility of explaining differences in forgiveness at the individual level.

In a subsequent step, the social constructions and the perceptions of the differences between individual-level and group-level forgiveness of individuals across three countries (Philippines, Japan, and Germany) are explored through a qualitative approach in Study 2 (Chapter 4). The main objective of Study 2 is the exploration how people from different cultural backgrounds articulate the meanings of forgiveness at the individual and group levels, and to understand the contexts in which forgiveness is granted or withheld.

Study 3 (Chapter 5) and Study 4 (Chapter 6) build on the insights gained in Study 2 with a focus on formerly victimized societies in a post World War II context. In Study 3, findings from three formerly victimized societies in Europe (France, Poland, and Russia) will be compared to findings from three formerly victimized societies in East Asia (China, Taiwan, and the Philippines) in relation to their willingness to forgive Germans and Japanese respectively. Study 3 and 4 are based on a theoretical framework outlined in this chapter, which will be elaborated in detail in Chapter 5 and Chapter 6 respectively. Study 4 examines the former perpetrators Germany and Japan regarding how they deal with the past in assessing their attitudes towards compensation and past related constructs.

In Chapter 7 all results are integrated and discussed, future research is suggested and the chapter closes with a concluding remark. 


\section{Chapter 3}

\section{Study 1: Socio-economical and socio-political contextual correlates of interpersonal forgiveness - A meta-analytical approach across 13 societies}

\section{Introduction and outline of Study 1}

The present study summarizes statistically previous research using the Enright Forgiveness Inventory (EFI) and explores the relationships of society-level means of interpersonal forgiveness as measured by the EFI with other constructs (e.g. psychological and contextual variables) using meta-analytical procedures. The guiding research questions were (1) whether there are differences between the societies and (2) whether contextual variables provide explanations for differences in interpersonal forgiveness between the societies. The main interest is the role of contextual variables and their influence on interpersonal forgiveness. Another interest is also the replication of relationships found at the individual level in studies at the country level ${ }^{1}$. Therefore, the focus of Study 1 is to investigate the impact of contextual variables and the possibility of explaining differences in forgiveness at the individual level. First, I will describe the EFI, followed by a discussion of potential society-level correlates. Subsequently, I will formulate the guiding research hypotheses based on this analysis, and discuss the usefulness of a meta-analytical procedure to assess and examine differences between societies.

The Enright Forgiveness Inventory (EFI)

The Enright Forgiveness Inventory (EFI; Enright \& Rique, 2000; Subkoviak, Enright, Wu, Gassin, Freedman, Olson, \& Sarinoapoulos, 1995) is the most - arguably the only - widely used self-report instrument that captures forgiveness as a moral response to a specific transgression at the interpersonal

\footnotetext{
${ }^{1}$ Country and society are used interchangeably.
} 
level. The EFI is based on the following definition of interpersonal forgiveness: "Forgiveness is a willingness to abandon one's right to resentment, negative judgment, and indifferent behavior toward one who unjustly injured us, while fostering the undeserved qualities of compassion, generosity and even love toward him or her" (Eniright \& Rique, 2000, p. 1). The EFI attempts to objectively assess the degree to which one person forgives another person for an unfair and hurtful deed inflicted on her- or himself. In general, the EFI consists of a set of 60 items with three subscales (affect, cognition, behaviour) with 20 items each. Each subscale is divided into 10 positive and 10 negative items. Every item is assessed on a 6-point Likert scale of agreement ( $1=$ strongly disagree; $6=$ strongly agree $)$. A sum score ranges from 60 (lowest) to 360 (highest) indicating and capturing key ideas about forgiveness: "the reduction in negative feelings, thoughts, and behavior and the development of positive feelings, thoughts and behaviors toward an offending person" (Enright \& Rique, 2000, p. 9). According to Enright and Rique (2000), forgiveness is a homogenous concept and therefore, the sum score over all 60 items assessing affect, cognition and behaviour should be used.

The EFI is self-administered and assesses current feelings, current thoughts and current behaviours toward someone who has inflicted harm on them and hurt them deeply and unfairly. Before the actual scale (labelled as an attitude scale for the participants) starts, participants are asked to recall a hurtful experience that recently occurred and to indicate the degree of hurt, who hurt them and whether this person is still alive, how much time passed since the injury and a description of the offense. Although these aspects are assessed, they are usually not reported. Then the participants are instructed to indicate to what degree they currently experience feelings, how they would act and what they think about the person who 
inflicted harm on them. Sample items ${ }^{2}$ for positive and negative feelings are: warm, kindness, happy, friendly, bitter, disgust, angry, cold. Sample items for positive and negative behaviours are: show friendship, help, treat gently, avoid, ignore, act negatively. Sample items for positive and negative cognitions are: nice, loving, wish the person well, evil, horrible, dreadful, condemn the person. The EFI has been used to assess the efficacy of therapeutic interventions and relationships to other psychological constructs (Coyle \& Enright, 1997; Subkoviak et al., 1995).

The benefits of forgiveness have been now documented (Toussaint \& Webb, 2005). According to previous research, the relationship between forgiveness and psychological well being, life satisfaction and happiness is positive (Karremann, van Lange, Ouwerkerk, \& Kluwer, 2003; Krause \& Ellison, 2003; Maltby, Day, \& Barber, 2005; Poloma \& Gallup, 1991; Rye, Folck, Heim, Olszewski, \& Traina, 2004; Toussaint, Williams, Musick, \& Everson, 2001). Yet, there is a lot of room for exploration. It is expected that a meta-analytical approach can provide further understanding of these relationships, since they will be investigated at the country level. The country-level is particularly interesting, since it can reveal context effects influencing interpersonal forgiveness. Building on previous results, I propose that interpersonal forgiveness is positively related to well being, life satisfaction and happiness at the country level (Hypothesis 1).

Enright and Rique (2000) claim that forgiveness is contextual, which means in their terminology that forgiveness is related to a specific unfair event. It is reasonable to argue that the context within which individuals interact and live can also contribute to an explanation of interpersonal forgiveness. It is argued that forgiveness contributes to successful reconciliation processes and may add to sustainable peace building in the long run (see Roe, 2007). Furthermore,

\footnotetext{
${ }^{2}$ I can only show some sample items, since the EFI is under copyright. Therefore, I am not allowed to reproduce the full scale.
} 
individuals do not exist in a vacuum; they are influenced by the social context and by the culture that they live in (Power, 2004). Therefore, the investigation of macro-level context variables in relation to forgiveness promises to be an interesting endeavor. Berry's ecocultural framework ${ }^{3}$ is useful for this task since it links socio-political contextual variables to individual-level variables (Berry, 1976, cited in Georgas, van de Vijver, \& Berry, 2004). Furthermore, Inglehart's (1997) proposition about post-materialism claims that newer generations are more socially and economically secure than older generations leading to a shift from materialistic to post-materialistic values (affluence of a society). The contextual environment may let forgiveness flourish as a more prosocial response to an interpersonal transgression.

The peacefulness of a society, economic and political indicators are referred to as socio-political context variables in the present analysis. Researchers who developed the Global Peace Index argued that 'if 'charity begins at home'- so might peace" (Vision of Humanity, 2007), meaning that when there is internal stability within a society it will contribute to the level of peacefulness. Therefore, one may argue that practising forgiveness in response to interpersonal transgressions may be one of these 'charities that start at home'. However, it is also arguable that practicing forgiveness requires a safe environment where there are no worries about survival due to conflicts. Recent research (Fischer \& Hanke, 2009) established robust links between the peacefulness of a society, values and country development. The value-peace link depended on the level of country development in this study, indicating that country development is a boundary condition (Fischer

\footnotetext{
${ }^{3}$ Berry's theoretical framework includes ecological factors, I tested the relationship between forgiveness and population density, low and high temperature, yielding a marginal significant correlation between forgiveness and population density $\left(r_{s}(9)=-.62, p=.07\right)$, a non-significant association between forgiveness and high temperature $\left(r_{s}(9)=-.03, p=.93\right)$, and a significant relationship between forgiveness and low temperature $\left(r_{s}(9)=-.66, p=.05\right)$. However, I will only focus on specific variables that are of interest for social scientists. Ecological factors are of less interest.
} 
\& Hanke, 2009). With the thought that forgiveness is often linked to reconciliation and peace-building (e.g. Staub et al., 2005), one can argue that if a society is able to provide a peaceful environment, it should increase the general tendency for people to show interpersonal forgiveness. Furthermore, it has already been argued that it is reasonable to associate the level and stability of democracy and human rights with an increase in expressions of positive emotions; and to associate instability with a decrease in expressions of positive feelings (van Hemert, Poortinga, \& van de Vijver, 2007). Based on these arguments I hypothesize that countries with strong and stable democracies, human rights, country development and peacefulness will show higher levels of interpersonal forgiveness (Hypothesis 2).

In a novel approach, Liu, Páez, Hanke, Rosa, Hilton, Sibley, et al. (2009) introduced a new inventory at the country level that predicted willingness to fight for one's country in the future at the individual level. This inventory was called Historical Calamities, and was based on the evaluations of events in world history across 30 countries. It included man-made and natural disasters (e.g. warfare, genocide, global warming). The inventory was based on the theoretical framework of social representations of history and identity, arguing that history provides raw materials that can function as a symbolic reserve. These materials are worked-up in communication processes guided by the political elites to define group boundaries and to legitimize ideologies (Hilton \& Liu, 2008; Liu \& Hilton, 2005).

Liu and colleagues argued that Historical Calamities is such a symbolic reserve that is used in political debates and influences the public (Reicher \& Hopkins, 2001). This research provided evidence that evaluations of historical calamities predicted the willingness to fight for one's country (countries with more negative evaluations were less willing to fight for their country). Liu's and colleagues' (2009) research was consistent with previous findings on perspectives 
towards politically approved violence and war (e.g. countries with more materialistic, collectivistic, and hierarchical values had less negative perspectives towards war; Basabe \& Valencia, 2007). Inglehart and Baker (2000) proposed a shift from traditional to materialistic and eventually to post-materialistic values. This shift is generally linked to a shift in the focus of the representation of war and its consequences with the emphasis on human suffering and the pointlessness of war (Rosoux, 2001). The negative representations of war and catastrophes and the consequences are captured by the evaluation of historical calamities. Furthermore, Liu and colleagues (2009) demonstrated that members of societies were willing to fight in order to achieve development. In line with this, it is reasonable to propose that the more negatively historical calamities are evaluated in a society, the higher should be interpersonal forgiveness levels among its citizens, (Hypothesis 3) since forgiveness can be cautiously thought of as the opposite of the willingness to fight.

In sum, two research questions will be addressed:

Research question 1: Are there differences in interpersonal forgiveness between societies?

Research question 2: What contextual variables account for differences in interpersonal forgiveness between the societies?

Furthermore, the following hypotheses will be tested:

Hypothesis $1\left(\mathrm{H}_{1}\right)$ : Interpersonal forgiveness should be positively related to well being, life satisfaction and happiness at the country level.

Hypothesis $2\left(\mathrm{H}_{2}\right)$ : Countries with strong and stable democracies, human rights, human development and peacefulness will show higher levels of interpersonal forgiveness.

Hypothesis $3\left(\mathrm{H}_{3}\right)$ : Societies that evaluate historical calamities more negatively should be higher on interpersonal forgiveness. 
I will now further describe the meta-analytical approach that I employed to answer the questions and to test the hypotheses.

\section{A cross-national meta-analytical approach}

A meta-analysis is a useful statistical technique that aims to answer an overall research question by systematically summarizing previous studies (Hunter \& Schmidt, 2003; Lipsey \& Wilson, 2001; van Hemert, 2003). A “meta analysis refers to the analysis of analyses ... the statistical analysis of a large collection of analysis results from individual studies for the purpose of integrating the findings" (Glass, 1976, p. 3). A meta-analysis has the advantage that results are summarized in a more refined manner, and at the same time helps prevent researchers from over-interpreting findings across studies (Lipsey \& Wilson, 2001).

Traditional meta-analytical approaches usually examine group mean differences and correlations between concepts. Dekker and Fischer (2008) outlined three arguments why these conventional approaches are problematic for the kind of studies my study falls into. Firstly, they argue that using these meta-analytical procedures limit the use of available information as only studies that actually made comparisons can be included. Secondly, they discussed the problematic use of group mean differences limiting the scope of understanding why there are differences and what these differences mean. Finally, they concluded that metaanalytical approaches in this form highly depend on prefixed comparisons of samples. Consequently, any further information about the context (e.g. peacefulness of a society, economic development) cannot be included in the analyses and direct testing of context effects is not possible (Dekker \& Fischer, 2008).

Dekker and Fischer (2008) suggested that these limitations can be overcome through using means directly (as demonstrated in Dekker \& Fischer, 
2008; Fischer \& Chalmers, 2008; Fischer \& Mansell, 2007). The main advantage with this approach is that it can handle the introduction of country as a level of analysis. I will follow the outlined suggestions, including using the means directly which allows evaluation of the differences more precisely, and employing the instrument-based approach outlined by van Hemert (2003). Using the means as the effect size is possible for meta-analytical approaches, since a mean would also apply to be converted into a standardized index making it comparable across studies and across societies (Lipsey \& Wilson, 2001).

According to van Hemert (2003) there are two options for conducting a cross-country meta-analysis: an instrument-based and domain-based approach. The domain-based approach focuses on one domain (e.g. intelligence) and gathers studies from a range of different instruments assessing the same domain or concept of interest allowing for a broader scope. An instrument-based approach focuses on a specific instrument, which is considered as a standard procedure. This approach is suitable for this study since the focus is on a specific instrument that captures interpersonal forgiveness.

Please note that meta-analyses of means are rarely reported, since it is not a meta-analysis in its traditional sense (Fischer \& Chalmers, 2008). However, a meta-analysis of means is feasible since it uses information from published studies. Furthermore, a meta-analysis of means employs the same statistical meta-analytical techniques used in conventional meta-analyses of correlations or group mean differences. The conventional term "effect size" referring to a meta-analysis with means can be misleading, but it can be argued since the effect size is the mean that the mean level is caused by the society people are residing in. In meta-analyzing the societal-level means obtained from published studies, it is possible to reveal society effects impacting on the means by examining societal characteristics (such 
as socio-political variables) that help explaining differences between societies which is in the scope of this study.

\section{Method}

\section{Literature search}

A PsycINFO search was conducted covering the period 1995 (year of the first EFI publication) to June 2007, searching for the keywords Enright Forgiveness Inventory and forgiveness. The search resulted in 54 references. Inclusion criteria were that (1) participants were older than 16 years of age, (2) were neither delinquent nor mentally disabled, (3) the studies were not clinical trials, and (4) that sufficient statistical information was reported (e.g. sum scores or mean scores).

Twenty articles that reported 26 independent samples met these criteria. Two articles included unpublished studies and another two were dissertations. Overall, the meta-analysis contained data from 6110 participants coming from 13 different societies. The majority of studies $(42.3 \%)$ were conducted in the USA (number of independent samples $k=11 ; N=2,010$ ). For each sample, the reported EFI sum scores or EFI mean scores were coded, what country, and (if reported) the Cronbach's alpha and demographic information (e.g. gender, age).

\section{Sample characteristics}

Participants were university students (26.9\%), college students and their same-sex parents $(38.5 \%)$, or the general population (34.6\%). Mean age for the overall sample was 32.9 years of age. Mean age for the student sample was 22.35 years, for the college students and their same sex parents the mean age was 35 years, the general population sample had a mean age of 38.24 years. The percentage of males in the overall sample was $44.34 \%$. 
Psychological variables, such as subjective well being (Diener, Diener, \& Diener, 1995), happiness (Veenhoven, 1999; World Value Survey, Inglehart, 1997), and life satisfaction (World Value Survey, Inglehart, 1997) at the country level were used to replicate the findings from previous research at the individual level (Karremans et al., 2003; Maltby et al., 2005; Rye et al., 2004). All country scores are taken from studies conducted at the country level (Diener et al., 1995; Veenhoven, 1999; World Value Survey, Inglehart, 1997). First, these indicators were factor analyzed revealing a single factor (Eigenvalue $=3.30$ ) which explained $82.45 \%$ of the variance. Therefore, after the indicators were $\mathrm{z}$-transformed I used mean scores to represent a well being factor.

A peace index was calculated consisting of the Global Peace Index (GPI) from 2007, 2008 and 2009 (Vision of Humanity, 2007, 2008, 2009) and Domestic Political Violence (DPV, van de Vliert, 1999) as context variable reflecting the peacefulness of a society. The GPI is based on 24 indicators from three categories: (a) ongoing domestic and international conflict, (b) country safety and security, and (c) militarization. Since the DPV is reflected in category (a), it was included in the overall Peace indicator. Therefore, the four indicators (GPI 2007 - 2009 and DPV) were factor analyzed yielding a strong single factor solution (Eigenvalue $=3.33$ ), which explained $83.15 \%$ of the variance. A mean score was devised which was based on z-transformed indicator scores.

I calculated a socio-economic indicator by factor analyzing the Human Development Index (HDI) scores from 1990, 1995, 2000, and 2004, Gross National Income (GNI) per Capita scores from 1990, 1995, 2000, 2004, 2005 and 2006 and Gross Domestic per capita (GDP) scores from 1990, 1995, 2000, 2004, 2005 and 2006. The principal components factor analysis revealed a strong single 
factor solution which explained $94.9 \%$ of the variance $($ Eigenvalue $=14.24)$. I calculated a mean score that represents socio-economic development based on ztransformed indicator scores.

A democracy indicator was used consisting of the Human Rights index for rights and freedoms by Humana (1986), by Freedom House (n.d., index of political rights and civil liberties), levels of democracy (Inglehart, 1997) in 1990 and in 1995, stability of democracy (Inglehart, 1997), and level of democracy as reported by van de Vliert et al. (1999), to capture a wider time span. These indicators were factor analysed with an oblique rotation and revealed a strong single factor solution (Eigenvalue $=6.38)$ explaining $91.10 \%$ of the total variance. Therefore, I recoded indicators that loaded negatively on the factors by multiplying the indictor with minus 1 and averaged the $\mathrm{z}$-transformed indicators into a single index.

Furthermore, to represent how societies evaluate the historical past I included the historical calamities indicator, which has been validated across 30 societies (Liu et al., 2009). Overall, these indicators corresponded to the well being of a society, and to socio-economical and to socio-political context variables at the country level. Table 3.1 provides an overview of included country scores. 
Table 3.1

Country-level indicators and corresponding country scores (Study 1)

\begin{tabular}{cccccc}
\hline Society & $\begin{array}{c}\text { Well } \\
\text { Being } \\
\text { Index }\end{array}$ & $\begin{array}{c}\text { Democracy } \\
\text { Indicators }\end{array}$ & $\begin{array}{c}\text { Peace } \\
\text { Indicators }\end{array}$ & $\begin{array}{c}\text { Socio } \\
\text { Economic } \\
\text { Indicators }\end{array}$ & $\begin{array}{c}\text { Historical } \\
\text { Calamities }\end{array}$ \\
\hline Austria & -.19 & .59 & .83 & .41 & 1.75 \\
Belgium & .78 & .76 & .70 & .43 & 1.80 \\
Brazil & -.28 & -.55 & -.42 & -1.72 & 2.12 \\
Hong Kong & - & -.55 & .31 & .29 & 2.66 \\
Israel & -.87 & .08 & -1.70 & -.10 & - \\
Korea, South & -1.46 & -.96 & .18 & -.66 & 2.56 \\
Norway & .68 & .74 & 1.29 & .91 & 1.81 \\
South Africa & -1.15 & -1.85 & -.98 & -1.71 & - \\
Switzerland & .94 & .72 & .88 & .83 & 1.75 \\
Taiwan & - & -1.18 & .09 & - & 2.55 \\
UK & .66 & .64 & -.31 & .35 & - \\
USA & .90 & .72 & -.79 & .97 & 1.98 \\
\hline
\end{tabular}

\section{Meta-Analytic procedures}

The mean score or sum score for interpersonal forgiveness from the EFI were used to calculate the effect sizes for the meta-analysis. Coded sum scores were converted into means by dividing the sum score by the number of reported items. All means were then standardized by dividing them by the response scale range (6-point scales), resulting in scores ranging between 0 and 1 . This type of standardization is useful in order to obtain a comparable metric of effect sizes (means), as one study used a different number of items (Dekker \& Fischer, 2008; Fischer \& Chalmers, 2008; Fischer \& Mansell, 2009). Therefore, the standardized metric derived from the reported sum scores and means are the effect sizes used for this meta-analytical approach.

Attention was paid to the sample size of each study, as bigger sample sizes, allow one to be more confident that the estimation of the population is precise. For this reason, a study that has a bigger sample size should carry more weight in a meta-analysis (Lipsey \& Wilson, 2001). Lipsey and colleague thus recommend 
weighing each effect size by the inverse variance. The inverse variance takes the standard error into account for it is a direct index of the precision of the effect size and it also provides confidence intervals (Lipsey \& Wilson, 2001). The criterion is that the smaller the standard error, the more precise the effect size will be.

In all analysis at the study level (or the effect size level) the effect sizes being used were inverse-variance-weighted, and mixed effects models were used in every ANOVA. The analysis focusing on associations between country-level indicators and forgiveness were conducted at the country level. For this purpose, I aggregated the standardized mean to the country level, in such a way that each score represented one country. Therefore, the country level data set had 13 cases in which the cases refer to each country.

Hence, I had two levels of analyses: the first level was the study level or effect size level, which evaluated variability and possible moderators to explain this variability, using effect sizes weighted by the inverse-variance as outlined above; and (2) the country-level in which the aggregated standardized effect size (the standardized mean) was used. I followed the recommendation of Fischer and Chalmers (2008) and used a mixed effect model since it allows generalizations beyond the studies that were included. A mixed effect model uses random-effects models, but additionally allows for systematically testing remaining variance to investigate study characteristics that go further than plain random variation (Fischer \& Chalmers, 2008). I used an inverse-variance weighted ANOVA macro as provided by Lipsey and Wilson (2001). 


\section{Results}

\section{Examination of publication bias}

A useful method of detecting whether a publication bias exists is the creation of a funnel plot in plotting the effect sizes against the sample sizes (Lipsey \& Wilson, 2001). Lipsey and Wilson argued that if a collection of effect sizes is unbiased there should be large variability between the small samples compared to large samples. Although there are relatively few studies in this meta-analysis, the scatterplot displays a funnel-shaped distribution indicating that there is more variability among the small samples compared to larger samples. Publication bias appears unlikely. Therefore, there it is unlikely that a publication bias affects the results reported below (see Figure 3.1).

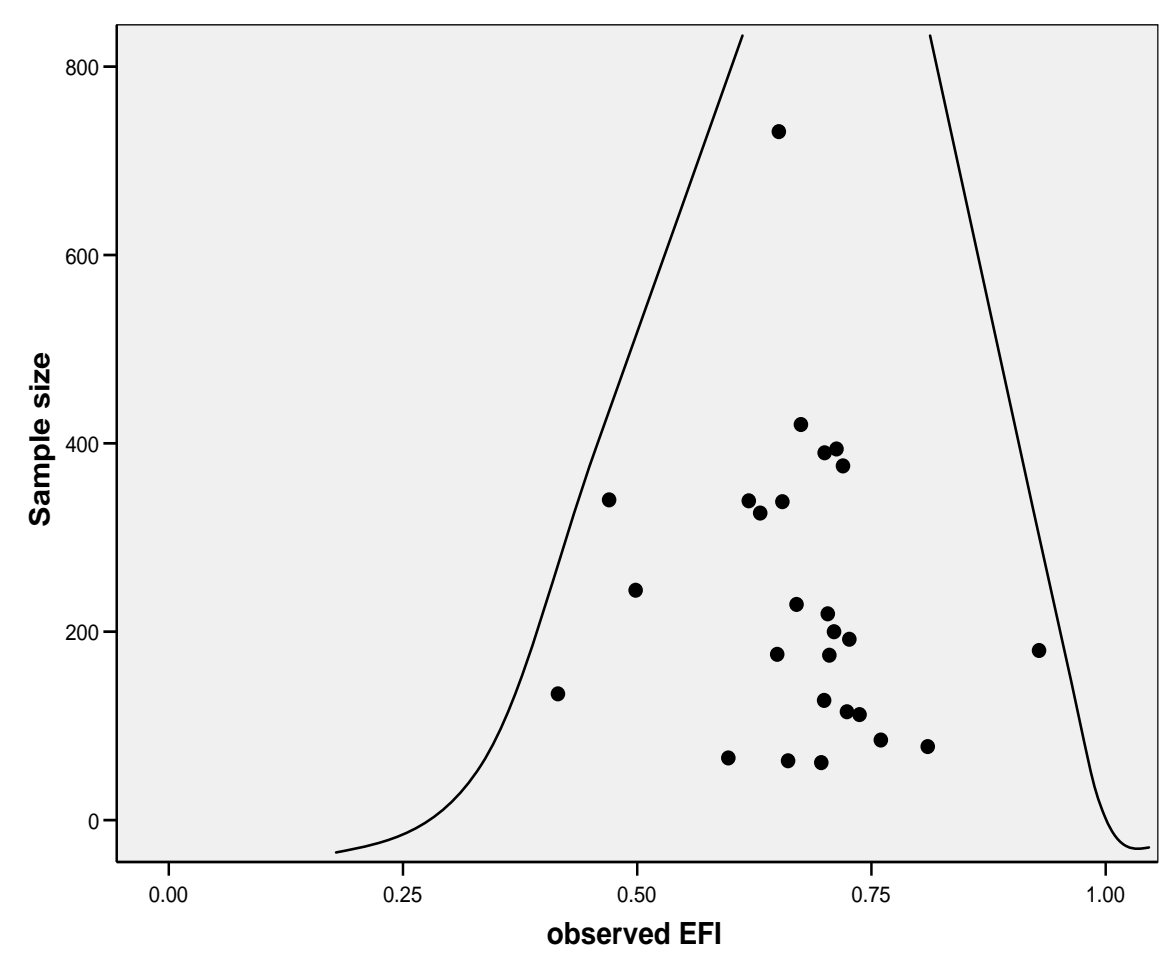

Figure 3.1 Funnel Plot of the effect sizes (EFI-mean) and sample size (Study 1) Interpersonal forgiveness across 13 societies

An inverse variance-weighted random effects model yielded an average mean of .68 (equivalent to an EFI sum score of 244.8) with a 95\% confidence 
interval of .62 - .73. Therefore, on average individuals were quite forgiving after interpersonal transgressions. The South African sample had the lowest forgiveness score and Switzerland the highest (see Table 3.2).

\section{The Q-statistics}

The Q-statistic is distributed as a $\chi^{2}$ and can be used for testing the assumption whether all effect sizes are similar in their estimation of the population mean. Therefore, if the Q-test is significant and the assumption of homogeneity is rejected, effect sizes are considered heterogeneous, which means that it allows for testing moderators to explain these differences. The calculation of the Q-statistic is based on three sums. The sum of the weights, the sum of the product of the weight and effect size, and the sum of the weights times the squared effect size.

A sample-size weighted mixed effects ANOVA revealed significant variability between societies $(Q(12,25)=168.62, p<.001)$. This finding answered the first research question: there were differences between societies on interpersonal forgiveness. Therefore, explanations of differences were possible through examining potential moderators by analyzing between study effect sizes variability and categorical variables, using inverse-variance weighted mixed effects ANOVAs. 
Table 3.2

Mean levels (effect sizes) per society based on mixed-effects model (Study 1)

\begin{tabular}{lcccccc}
\hline Society & $\begin{array}{c}\text { Studies } \\
(\mathrm{k})\end{array}$ & $\begin{array}{c}\text { Sample } \\
\text { Size (n) }\end{array}$ & EFI & SE & -95\%CI & \multirow{2}{*}{$+95 \% \mathrm{CI}$} \\
\hline Austria & 1 & 376 & .72 & .07 & & \\
Belgium & 1 & 731 & .65 & .07 & & \\
Brazil & 2 & 590 & .71 & .05 & .61 & .80 \\
Hong Kong & 1 & 63 & .66 & .07 & & \\
Israel & 1 & 176 & .65 & .07 & & \\
Korea, South & 2 & 392 & .61 & .05 & .52 & .71 \\
Northern Ireland & 1 & 340 & .48 & .07 & & \\
Norway & 1 & 115 & .72 & .07 & & \\
South Africa & 1 & 134 & .42 & .07 & & \\
Switzerland & 1 & 180 & .93 & .06 & & \\
Taiwan & 1 & 339 & .62 & .07 & & \\
United Kingdom & 2 & 664 & .59 & .05 & .50 & .68 \\
United States of America & 11 & 2010 & .72 & .01 & .69 & .74 \\
\hline \multicolumn{7}{c}{} \\
\hline
\end{tabular}

Explanation of differences in exploring possible moderators at the study level

The homogeneity-test indicated that there was variability between the societies on interpersonal forgiveness and this justified the examination of potential moderators (Hedges \& Olkin, 1985; Lipsey \& Wilson, 2001). Therefore, I tested categorical moderators relevant to the sample and context. Population (coded as 1 $=$ university students, $2=$ college students and their parents, $3=$ general population), age ${ }^{4}$, gender proportion (median split, coded as $1=$ below $50 \%$ of males, $2=$ above $50 \%$ of males) were tested as potential moderators for explaining differences.

Contextual moderators included region coded as $1=$ Europe, $2=$ Asia, $3=$ South America (Brazil), $4=$ USA, potential conflict regions were coded as $1=$ yes, $2=$ no (Northern Ireland, Israel, and South Africa were deemed as potential conflict regions), religion was coded as $1=$ Christian and $2=$ non-Christian). The moderators were tested using inverse-variance weighted mixed effects ANOVAs.

\footnotetext{
${ }^{4}$ I used a median split to convert the age into two categories $(1=$ below $34.5,2=$ above 34.5$)$. I tested the same with the categories "adolescents" (under 20) and "adults" (over 20) and yielded a similar non-significant result: $Q(1,19)=.88$, n.s.
} 
There was no significant effect due to the population $(Q(2,25)=.17$, n.s. $)$, due to age $(Q(1,20)=.04$, n.s. $)$, but due to gender $(Q(1,17)=5.73, p<.05$. $)$. There are inconsistent findings in the literature regarding gender differences on forgiveness (e.g. Hewstone et al., 2004). However, this result indicates that when there were fewer males in a sample, the forgiveness scores were slightly higher. Region was not significant $(Q(3,25)=4.7$, n.s. $)$, and religion $(Q(1,25)=.36$, n.s. $)$ was not either. On the other hand conflict was a significant moderator $(Q(1,25)=12.88, p$ $<.05)$. Those countries categorized as potential conflict regions showed significantly lower forgiveness scores than those in non-conflict regions. Therefore, in response to research question 2 (What contextual variables account for differences in interpersonal forgiveness between the societies?): gender distribution in the samples and conflict potential are prospective explanations for differences in interpersonal forgiveness scores.

\section{Correlations at the society level}

The next step was to test the hypothesized associations. For this purpose, I aggregated the standardized means and conducted correlations at the country level (see Table 3.3). Due to the limited number of societies, nonparametric Spearman rank order correlations were used, as suggested by Dekker and Fischer (2008). The correlation can further provide explanation as to why countries differed in interpersonal forgiveness scores. 
Table 3.3

Correlation between EFI means and Well-being Index, Democracy Indicators, Peace Indicators, Socio-Economic Indicators, and Historical Calamities at the country level

\begin{tabular}{lccccc}
\hline Indicator & 1. & 2. & 3. & 4. & 5. \\
\hline 1. EFI & - & & & & \\
2. Well-being Index & $.62^{*}$ & - & & & \\
3. Democracy Indicators & $.58^{*}$ & $.88^{* *}$ & - & & \\
4. Peace Indicators & $.62^{*}$ & .48 & $.54^{\ddagger}$ & - & \\
5. Socio Economic Indicators & $.55^{*}$ & $.84^{* *}$ & $.87^{* *}$ & 54 & - \\
6. Historical Calamities & $-.68^{*}$ & -.54 & $-.67^{*}$ & -.55 & -.45 \\
\hline Note. $\mathrm{N}=12$ (societies) ${ }^{5},{ }^{\sharp} p \leq .08, * p \leq .05, * * p<.01$ & & &
\end{tabular}

The well-being index was significantly positively associated with the EFI interpersonal forgiveness score at the country level. If well-being was high in a society, the higher was the interpersonal forgiveness score. Therefore, a replication of previous findings at the individual level was successful, supporting Hypothesis 1. This result also points to functional equivalence, as I found a similar pattern to the individual level at the country-level (van Hemert, 2003). However, functional equivalence can only be cautiously suggested, since no indicators other than well being were included.

A strong and significant correlation between the democracy indicator and interpersonal forgiveness at the society level was found. That means the more democracy and human rights a society has, the higher forgiveness score. Furthermore, a significant correlation between the Peace index and interpersonal forgiveness was found. The more peaceful a society was, the more forgiveness was shown at the interpersonal level. Moreover, there was a marginal significant association between the socio-economic indicator and interpersonal forgiveness. That means when a society is able to establish a certain socio-economic development, the higher the interpersonal forgiveness score. The results support

\footnotetext{
${ }^{5}$ Since there is no country-level data available for Northern Ireland, it was excluded from the subsequent analyses.
} 
Hypothesis 2 that countries with strong and stable democracies, human rights, country development and peacefulness are meaningfully linked with higher levels of interpersonal forgiveness.

There was an interesting significant association between the evaluation of historical calamities and interpersonal forgiveness. In line with Hypothesis 3, the more negatively historical tragedies were evaluated, the higher the interpersonal forgiveness score.

In sum, I discovered significant variability between the countries. These differences can be explained by gender distribution of the samples and conflict potential. Furthermore, the well-being of a country was significantly associated with interpersonal forgiveness. The same associations were found for the stability and level of democracy, state of human rights and socio-economic development of a country and the levels of interpersonal forgiveness. An interesting finding is the role of the shared representation and evaluation of negative historical events, which was negatively associated with forgiveness at the country level.

\section{Summary and discussion}

The main objectives of this study were to explore differences between the

countries in interpersonal forgiveness using a cross-country meta-analytical approach and the examination of categorical contextual variables (moderators) to provide explanations for these differences.

First, detected variability between the societies can be explained by gender distribution and potential conflict regions. Therefore, the analysis at the study level suggested that the higher the proportion of male participants in the included studies, the lower the report of forgiveness. Furthermore, coded conflict regions (South Africa and Northern Ireland) had lower forgiveness scores, which points to context relevant factors that affect interpersonal forgiveness. Whether the samples 
were predominantly Christian did not influence the interpersonal forgiveness scores, which is in line with previous research (Worthington, 2005).

The findings also suggested that interpersonal forgiveness is positively associated with well-being at the country level. This result was anticipated, as it replicates results found at the individual level. This finding is highly encouraging since it shows the potential benefits of forgiveness at the country level. Whereas previous research only examined the positive benefits of forgiveness at the individual level, this analysis shows that forgiveness is associated with positive effects across societal contexts. Of course, the direction of causality in these effects cannot be ascertained using correlational methods.

More insight can be gleaned from the results of the examination of socioeconomic and socio-political context variables. These results suggest that in order to encourage forgiveness as a moral response to harm done, there needs to be some stability in a society and a certain level of development. In post-conflict societies, there is a heightened need for safety (Staub, 2004). Unsafe environments in which the emphasis is on survival (Inglehart, 1997) may reduce the likelihood of perceiving forgiveness as a response option towards transgressions, since many transgressions are also based on group-based struggles over power (e.g. Northern Ireland or South Africa). With the notion of forgiveness as a charity starting from home within a country, forgiveness could also foster cooperation and increase the economic and developmental vitality across societies, which would suggest forgiveness as a possible engine for democracy and social stability in a society.

An intriguing finding was the association between the evaluations of historical calamities and forgiveness. The more negative the evaluations were, the more likely forgiveness was reported. Why is that interesting? The results are in line with assumptions made by of social representations theory in general 
(Moscovici, 1988), and specifically social representations of history (Hilton \& Liu, 2008; Liu \& Hilton, 2005), that shared representations in a society have an influence on the individual. Liu and colleagues (2009) argued that a shift to a postmaterialistic society in line with Inglehart (1997) is also associated with a shift to more negative representations of war and its consequences. Therefore, the relationship between the evaluation of historical calamities and forgiveness adds an important component in order to understand how forgiveness is influenced by shared beliefs at the country level about the course of history.

However, all the correlation results should be regarded with some caution, since they do not allow causal conclusions. Nevertheless, these results point to a linear relationship between general well being, democratic and socio economic development and forgiveness. The question is whether this means that the only matter we should worry about is that each society has a stable democracy, human rights, and high socio-economic development. Is this enough to ensure that forgiveness is promoted and encouraged?

There are several limitations for the current analysis. The country level indicators were derived across multiple time points and diverse samples. Therefore, the used contextual correlates also captured a particular period; however, by doing this it is likely that particular temporal patterns were missed. The restricted number of countries is also a limitation. I had only 13 countries and limited number of samples (except for USA). This is a limiting factor for this meta-analysis' ability to explain differences between societies. However, although I had samples from a relatively small number of countries $(\mathrm{N}=13)$, the reported correlations at the country level are relatively large.

What can be said for sure from the results of the moderator analyses is that the gender proportion of participants and the conflict potential in a society seemed 
to matter. Other context variables, such as the economic vitality (providing a safe environment free from survival fears), democratic rules (signifying transparency or certainty about processes), have an influence on forgiveness as a practice as demonstrated in this study. Furthermore, these results suggest that forgiveness might have a relation to modernity and needs a certain level of stability within a society in order to grow.

This study provides some tentative evidence that contextual variables at the macro-level need more consideration, as they can potentially influence the tendency to forgive in response to an interpersonal transgression. If this metaanalysis already showed that there are substantial societal variation on interpersonal forgiveness; how much more variation will apply for the intergroup contexts? In order to facilitate forgiveness, it is important to understand how different cultures respond to this concept and how they perceive forgiveness. This will be the focus of Study 2. In the following study, I employ a qualitative approach to capture the social reality of individuals in different societies in relation to forgiveness as a practice at the individual and at the group level. Most importantly, it will focus on what distinguishes the two levels from one another and whether contextual variables are articulated as a way to draw the line between the two concepts. 


\section{Chapter 4}

\section{Study 2: Qualitative exploration of intergroup forgiveness}

\section{Introduction and outline of Study 2}

The aim of this study is to explore the concept of intergroup forgiveness through a qualitative approach. The meta-analysis in Chapter 3 provided empirical evidence that interpersonal forgiveness is partly shaped by contextual variables. This study focuses on the clarification of similarities and distinctions between forgiveness at the interpersonal versus at the intergroup level. It is anticipated that the findings will help to determine important concepts within the forgiveness process.

Previous survey-based research (Denton \& Martin, 1998; Kanz, 2000; Kearns \& Fincham, 2004; Mullet, Girard, \& Bakhshi, 2004) about how people conceptualize interpersonal forgiveness revealed some inconsistent findings signalling the need for more qualitative approaches to examine perspectives on forgiveness The way people outside the academic world conceptualize forgiveness seems for the most part similar to the ways forgiveness researchers conceptualize forgiveness (Kanz, 2000): reconciliation is a necessary part of forgiveness and forgiveness may be important and effective for therapeutic interventions. Some differences were found as well The participants viewed forgiveness as causing emotional problems whereas theorists proposed that only pseudoforgiveness ${ }^{6}$ can cause such problems and not true forgiveness (Kanz, 2000).

Mullet and his colleagues (2004) examined conceptualizations of forgiveness by laypeople and whether there is an agreement between accounts in the literature and people's views on interpersonal forgiveness. Participants viewed

\footnotetext{
${ }^{6}$ Pseudoforgiveness occurs when individuals think they have forgiven and they actually have not.
} 
forgiveness and reconciliation as separate constructs. Moreover, participants did not equate forgiveness with an end to resentment. Participants perceived forgiveness as being more than a dyadic process which is not consistent with the literature (see Worthington, 2005), but with survey-based research conducted with clinical psychologists (Denton \& Martin, 1998). Mullet and colleagues (2004) research showed that offender can be - besides being known by the offended unknown or abstract institutions. Furthermore, the process of forgiveness is difficult and after forgiveness is granted it may not change the future behaviour of the offender.

A prototype analysis ${ }^{7}$ of forgiveness (Kearns \& Fincham, 2004) revealed initial clusters of descriptions: affect (e.g. kindness, sadness), behaviours (e.g. talking, hugging), cognitions (e.g. understanding) and expectations (e.g. continuance of relationship). This research revealed that the conceptions of forgiveness by laypersons are similar to theoretical considerations of forgiveness. However, there were also some differences between laypersons' views and expert views. The participants disagreed with experts on what forgiveness is not. While experts excluded condoning, excusing or forgetting about the offense, the participants thought that these attributes belong to forgiveness. Similar to Kanz (2000), but inconsistent with Mullet and colleagues (2004), participants in this study perceived reconciliation being an important part of forgiveness. Reconciliation indicates a coming together of the parties again. The authors argue that if laypeople view reconciliation as an integral part of forgiveness, the likelihood to forgive is lower than if people view reconciliation as separate from forgiveness. Reconciliation indicates a coming together of the two parties and for

\footnotetext{
${ }^{7}$ In a prototype analysis participants identify features of a concept via free listings. In a subsequent step, the identified features are rated in order to determine their relevance or centrality for the concept.
} 
some people the idea to face the aggressor is threatening. This kind of reasoning about forgiveness makes it difficult to implement forgiveness into interventions.

Research (e.g. Neto et al., 2007a; Neto, Pinto, \& Mullet, 2007b; Kadima Kadiangandu \& Mullet, 2007; Kadima Kadiangandu, Gauché, Vinsonneau, \& Mullet, 2007) has been conducted in an attempt to conceptualize intergroup forgiveness. This research is scarce and deserves attention. Kadima Kadiangandu and colleague (2007) and Neto and colleagues (2007a, 2007b) examined a rarely investigated context. They researched African perceptions of intergroup forgiveness in the troubled African states of Congo, Angola, Guinea-Bissau and Mozambique. Their survey-based approach revealed that the concept of intergroup forgiveness made sense to all participants (general population samples). The survey was compiled based on accounts in the literature about political forgiveness (e.g. Digeser, 2001; Shriver, 1995). The participants agreed that the process needs to be a democratic, public one, that intergroup forgiveness does not necessarily depend on reparation or compensation and that the aim of the intergroup forgiveness process is reconciliation. The authors also conducted the same research in EastTimor with similar results.

Overall, the findings regarding laypersons' conceptualizations of interpersonal forgiveness overlaps for the most part with accounts in the literature. This might be due to the fact that a survey has been constructed on the basis of existing conceptualizations of interpersonal forgiveness and the assessment of the agreement on them. Therefore, one of the shortfalls of this kind of research was that ideas from the literature were imposed in form of surveys onto the participants. Except for the prototype analysis, participants got confronted with pre-formulated ideas about forgiveness. Nevertheless, the research on conceptualizations of intergroup forgiveness revealed that this concept is meaningful for people who 
suffered during conflicts. Therefore, it was not possible to discover other concepts than those in the literature. However, other concepts that might be important for the overall forgiveness process could be discovered through using another method. Furthermore, the conducted research fell relatively short on differentiating interpersonal from intergroup forgiveness.

The qualitative approach for this study was chosen in order to prevent to impose predetermined ideas of forgiveness and to catch the social reality of the interviewees. It is expected to find forgiveness related concepts (e.g. apology) which have been articulated in the literature before. Furthermore, there is a probability that the interviewees talk about concepts that have not been mentioned in the literature before, since no concepts are imposed onto the participants. I also anticipate discovering how people coming from different cultural backgrounds articulate the differences between interpersonal and intergroup forgiveness. The expression of the differences may be helpful to determine what intergroup forgiveness entails.

\section{Method}

\section{Participants}

Participants were recruited by a contact person who approached them and introduced the topic of the interview (the snowballing method). Fifteen people from three countries (Germany, Philippines and Japan) were interviewed. In total, there were six female and nine male interviewees. The German, Filipino and Japanese interviewees comprised of two female and three male participants. Their ages ranged from 25 - 35 years $(n=9)$, to 36-45 years $(n=4)$ and over 46 years $(n=2)$. The average age for the German participants was 32.6 years, for the Filipino participants 36.2 years and for the Japanese 32.8 years. I had two criteria to decide 
which population should be included in this study. Participants should be older than 25 years of age, but younger than 60 years of age, because I followed the rationale of Govier's (2002) rationale of conducting research with 'tertiary victims', which refers to victims who have not directly experienced the past wrongdoing. This reduces the risk of evoking traumatic experiences (Kadima Kadiangandu \& Mullet, 2007). Moreover, interviewing people belonging to former perpetrator nations above this age limit could result in overly defensive reasoning about forgiveness at the group level due to a direct connection to the past (Rensman, 2004). Therefore, participants were the third or second generation after World War II (Germany, Philippines, and Japan), to ensure that they spoke about forgiveness relating to this topic at the group level, rather than at the individual level.

Germany and Japan were former perpetrator nations and the Philippines were a victimized nation. Although it would have been desirable to carry out interviews with people from a formerly victimized European nation (e.g. Poland), it was not possible to carry these out, since the interviews would have been needed to be carried out in a different language other than the researcher's language of German or English.

In the beginning of the semi-structured interview, demographic questions were asked and all Filipino participants identified themselves with Roman Catholic religion, whereas the German participants stated that their belief system was influenced by Christian principles, but identified themselves as being atheists or agnostic. The Japanese participants did not identify with either a religious or a philosophical orientation, but stated that they are influenced by Buddhism principles. 
In extracts to follow, interviewees were identified as $P$ for Philippines, $G$ for German and $J$ for Japanese. For indicating short pauses I used “(.)”, for long pauses “(...)", for unclear passages “..." and for omission of irrelevant passages for the theme within the speech to shorten the overall passages relevant to the theme.

\section{Interview schedule}

Interviews were conducted using a semi-structured interview schedule, which gained approval by the School of Psychology, Human Ethics Committee at Victoria University of Wellington, New Zealand. At the onset of the research process, major questions of interest centred on experiences of forgiveness at the individual and intergroup level (e.g. "Please describe a situation where you felt unjustly hurt or harmed", or "Has anything happened in the past to your national or ethnic group that might require forgiveness? Please describe what this might be." See Appendix A for complete overview of the interview schedule.

All questions were open-ended, except the demographic questions, which were asked at the beginning of each interview. The questions were adjusted during the interview and questions were occasionally skipped depending on the extent that participants were willing to share their experiences of forgiveness, and depending on whether some issues were already addressed in earlier questions. This procedure allowed the participants to have control and to direct the interview towards unanticipated topics, and for the interviewer to guide the conversation flow in a natural manner.

The interviews in the Philippines and in Japan were conducted in English, because English is the official language in the Philippines and because the interviews in Japan were conducted in university settings where students and staff were capable of communicating in English to an interviewer who was not 
conversant in Japanese. The German interviews were conducted in German as I am German myself.

\section{Procedure}

When the participants agreed to take part in this interview on a voluntary basis, the interviewees chose the venue they felt most comfortable with and a date was set for the interview. The nature of the research was explained, and participants were given an opportunity to ask questions. Before the interview started, participants were asked to sign a consent form if they were willing to participate in the study. With the agreement of the participants, the interviews were recorded for transcription and analyses. The duration of the interviews ranged from 25 to 54 minutes, taking an average of about 40 minutes. The German interviews were conducted in German, recorded and then transcribed and translated into English by me. The English transcripts of the interviews were verified with a bilingual German-English speaking postgraduate student in order to ensure a match between the English version of the data and the German one. The Filipino and Japanese interviews were conducted in English, recorded and transcribed.

The interviews were recorded using an Olympus WS-200S Digital Voice Recorder and a Samsung YP-U2 Digital Audio Player and Voice Recorder. Sound files were transcribed using Adobe Audition software. Transcripts were coded using MAXQDA software.

\section{Analytical strategy: Retroductive thematic analysis}

The corpus of data consisted of 65,000 words. After the interviews were transcribed, a form of thematic analysis detailed by Braun and Clarke (2006) was used. In general, thematic analysis, according to Braun and Clarke (2006), seeks to trace for the most widespread and main themes within the data. The main themes in 
turn are able to embody a summary of the whole data set in form of some phenomenon, process, or consistent 'narrative' (Braun \& Clarke, 2006; Grbich, 2007). Many other data reduction techniques within qualitative research methods may also be used (e.g. content analysis or grounded theory). However, thematic analysis (Braun \& Clarke, 2006) seemed the most appropriate technique for the study because of the flexible nature of thematic analysis, which suited the high complexity of the data. This complexity was due on the one hand to the translation of the interview transcripts from German (the native language of the participants) into English, and on the other hand due to the circumstances that the Japanese interviews were conducted in English. Thus, the analysis had to deal with an English version of the original interview which was already an interpretation of the participants' descriptions (in the German case); or with an English version that did not necessarily flow as it would have, had it been conducted in participants' native (Japanese) language. Therefore, techniques more reliant on the exact nature of the utterances were not appropriate. Given the breadth of topics examined and the relatively small and diverse samples, the data would have been insufficient for more systematic and comprehensive techniques. Considering that to address the complexity of a corpus, the analysis of the data should always go for the broader meanings and concepts (Cronin, 2006); thematic analysis was selected as the most appropriate analytical strategy.

Furthermore, I followed a retroductive analytical strategy (Glynos \& Howarth, 2007) which combines deductive and inductive analytical strategies. This was because although I did not formulate theories a priori, I was guided by my $a$ priori knowledge about the topic of "forgiveness", which thus influenced the choice of questions for the semi-structured interview schedule and the analysis. While inductive approaches formulate generalizations and theories based on 
multiple observations, and deductive approaches deliver empirical evidence to confirm a theory, retroduction is a more open-ended approach that allows researchers to make assumptions about what could be the case (Glynos \& Howarth, 2007). This makes it a very flexible approach that suited the flexibility of the proposed thematic analysis.

Following the guidelines of thematic analysis (Braun \& Clarke, 2006) the material was read and re-read and initial ideas were noted. This allowed initial codes to be identified, and for the data to be resorted and collated according to these codes. Subsequently, the identified main themes were mapped.

I acknowledge that I employed an insider's perspective regarding the interviews conducted with German participants, because I have been socialized in Germany. At the same time I had an outsider's perspective regarding the other two countries. Therefore, I considered my insider-outsider subjectivities on the presented perspectives as I conducted the analysis. According to Greenfield (2000) it is logically impossible to be truly observer-independent and perfect objectivism is not possible. Since from a psychological perspective each participant is an expression of culture (Bruner, 1990), it is expected to capture the meaning and understanding of forgiveness across participants coming from different cultural backgrounds reflecting the cultures respectively.

\section{Results and Discussion: Contextualized intergroup forgiveness}

In the following section, I describe the overarching themes labelled as "Facets of Intergroup Forgiveness", “Antecedents of Forgiveness", and "Outcomes of Forgiveness". The core themes are the antecedents of forgiveness, which contribute to the understanding of the forgiveness process. 
Facets of Intergroup Forgiveness - distinguishing interpersonal from intergroup

forgiveness

How did the participants distinguish between the individual and group

level?

Overall, Filipino and German interviewees conceptualised forgiveness at the individual level as an everyday practice and perceived it as easier to apply than forgiveness at the group level. Japanese interviewees held different views about forgiveness and did not consider it as a useful conflict resolution strategy.

This Filipino participant talked about forgiveness in an interpersonal setting as a simple endeavour because only two persons are involved and have to deal with it; contrary to forgiveness at the intergroup level:

"I think it is quite simple for two persons for a relationship because it is just a simple relationship it is just one person involved but whenever there are more than one person involved I think it becomes more and more complicated...the more people involved the larger the group I think it is harder to achieve forgiveness. Complete forgiveness I think or the ideal sense of forgiveness" [P2].

How can a group forgive completely? This question came up during some interviews and interviewees said that the problem at the group level was the variety of opinions. Another Filipino interviewee commented on the complexity of grouplevel forgiveness, with the majority influence and politics leaving people uncertain about when 'forgiveness' towards the perpetrator group is socially acceptable:

"Well in the personal level there are there aren't um there are no other people concerned or involved it's just you and me ... you know I hurt you... you forgive me or not so it's just up to you and what you know where in your group your community and your nation there all these other people and sometimes your decisions will tend ... you know tend to move or sway to the decisions of others ...you know it's like it's like the elections you know people vote not to forgive and okay might as well not so...It can influence other people can influence your decision even if you want yourself to forgive you know other people can change your mind but at a personal level it's you and the person involved" [P3]. 
At the intergroup level, forgiveness can be influenced by public opinion, so there is no clear closure. According to Liu and Hilton (2005) a wound can be re-opened as a political issue and therefore be used by political leaders to follow and find support for their agenda. Consistent with this formulation, with regard to groups there is the notion of forgiveness being a bilateral process (if only one side has been victimized) or mutual process (if both sides were perpetrators at some stage during the conflict). This is rather than forgiveness being a unilateral matter, as can be the case in forgiveness at an individual level:

"Forgiveness on a personal level is ... easier, because you only speak of your own ... you don't, it's you, if I forgive this person important to me, if I decide to forgive another person, it's my conscious decision ... when you talk of nations or groups, it's harder and as I said it takes a lot of effort because you are talking about history, you are talking about ... yeah, you bring with you history probably of ... injustice what has been done and I don't know perhaps it can also be between religions, like Muslims and Christians, you are talking about this level, you are talking about really values that are engrained already then it's a little difficult for you to collectively decide to forgive when we already been programmed into thinking that this group or this nation has done us wrong" [P4].

The preceding extract furthermore asserts that intergroup grievances may become part of a cultural or religious script that is engrained within people's consciousness or identity (e.g. Rata, Liu, \& Hanke, 2008). Similar opinions were reflected among the German interviewees. The major distinction between the two levels is the complex and uncertain nature of forgiveness at the group level.

"Well, I think at the individual level you are able to clearly determine whether it is forgiven or not, ... whereas in groups you are not able to clearly say this" [G3]

and

"In a group with 50 people it is useless, if only 3 want to undergo the forgiveness process while the rest does not want to engage in it" [G1]. 
In order to reduce the uncertainty at the group level, it is important to have someone who can represent the group in the forgiveness process - especially, if one's own group (here a Japanese participant) has to carry the burden of the past:

"Of course a representative can forgive as politician, but if people feel as a country, as a kind of common group in which each member share psychological traits, it is very difficult to forgive. But if people feel as a (...) common goal group, maybe it is possible to forgive (...)" [J5].

Overall, there was a common understanding among interviewees that forgiveness has a certain value, benefits, and is important. However, the Japanese interviewees regarded the process of forgiveness as relatively irrelevant for conflict resolution. Furthermore, forgiveness seemed to have negative connotations, as the concept was mainly associated with potential costs regarding the whole process. Nevertheless, forgiveness seemed to have at least a utilitarian value among the Japanese interviewees - but no moral connotations as following extract by a Japanese participant displays:

“(...) Basically I try to forgive someone, because forgiveness sometimes makes better relationships with him or her so in that sense I think forgiveness has some kind of benefits. Yeah (...) the situation in which someone hurt me is very rare for me so (...) I changed my mind. Sometimes I feel or I think the apology is a real one a sincere one (.) then (...) I would apologise no no I would forgive him. But even in that case I would try to make him or her apologise more and then I would forgive them (...)Yeah yeah so I think the expression of apology is needed repeatedly and then I would forgive" [J5].

If forgiveness was considered among the Japanese participants, it was surrounded by heavy conditions and obligations. Theoretically, these articulations are in line with the development model of forgiveness (Enright, Santos, \& AlMabuk, 1989; Enright, Gassin, \& Wu, 1992), which is modelled after Kohlberg's (1976) model of the development of moral reasoning and has six stages. Revengeful 
and conditional forgiveness are the lowest stages of forgiveness; the middle stages are exceptional forgiveness and forgiveness as social harmony: and the highest stage is forgiveness as love and is marked by its unconditional character. The extract corresponds with the stage of conditional forgiveness in which forgiveness is only granted if an apology or other forms of restitutions have been repeatedly received (Enright \& the Human Development Group, 1991, 1998). Furthermore, the extract marks also that forgiveness is an instrumental means, since the relationship could be beneficial in the future.

In sum, most interviewees understood forgiveness as an important and meaningful concept that many of them applied as a conflict resolution strategy in everyday life. In general, the participants attributed a certain positive value to forgiveness at the individual level, which is in concordance with previous research (McCullough, 2001; Enright, 2001). The participants were able to relate to forgiveness when thinking of individual situations. The Japanese participants repeatedly emphasized high conditions that have to be met in order to grant forgiveness when dealing with interpersonal conflicts. Avoidance was another response to offences ("I avoid them (conflicts) as much as possible" [J2]), and an association between shame and being in a conflict were mentioned by the Japanese interviewees:

"I think it is a shame to tell other people I have trouble I have conflict with someone. I don't want to say to other people or complain about that because I think having conflict having problem is not smart" [J3].

This avoidance approach to dealing with conflicts is echoed in the literature (e.g. Ohbuchi \& Takahashi, 1994). At the intergroup level the Japanese interviewees did not personalize the forgiveness process, suggesting that at this level it should be institutionalized. Although one should be very cautious about inferring group level differences in the articulation of specific aspect of 
forgiveness with such a small sample, it is still striking to find differences at this particular point. Referring to the individual level, the Filipino and German interviewees highlighted the benefits of forgiveness and the Japanese interviewees emphasized potential costs. These conceptualizations of interpersonal forgiveness can have an impact on how intergroup forgiveness is perceived and also how important forgiveness is perceived at the intergroup level.

Forgiveness in an interpersonal setting is described as something simple because only two persons have to resolve the conflict. In a group setting, forgiveness is perceived as being very complex because more people are involved. Therefore, forgiveness is more complicated and uncertain. The extracts portray the difficulties that arise when dealing with groups. One difficulty is that groups are perceived as variable and not as a stable entity. For example, one part of a group or a representative of a group can offer a sincere apology while another part may be sincerely opposed to this. The perceived control over the situation is higher in interpersonal settings than in intergroup settings. Strong communication processes seem to be necessary to approach and appreciate both parties and to gain an understanding of both sides of the story. Furthermore, group-level forgiveness requires a balanced process that involves each party approaching each other under optimal conditions (Allport, 1954), in order to achieve procedural fairness and reduce perceived unfairness and helplessness. The following excerpts portray these opinions.

\section{Antecedents of Forgiveness}

This theme was identified as being crucial, since elements such as 'apology', 'collective emotions and memories', 'representations of the past and historical closure', 'severity of offence' were identified and partially in line with previous research (e.g. Worthington, 2005). The following extracts for this theme 
are at the individual as well as intergroup level since it was easier for the participants to relate to the process of forgiveness at the individual level rather than the intergroup level.

Apology

This subtheme describes how the interviewees' perspective on apologies. The interviews conveyed how apologies are important for the victimized party, and that they are difficult for the perpetrator party to issue, particularly when the misdeed has happened in the past. The perceived sincerity of an apology is dependent on several other factors, which have a different emphasis at the interpersonal and intergroup level. These factors are portrayed by extracts in the following sections.

This following excerpt by a Filipino participant illustrates the dilemma of receiving an apology that includes compensation and not being able to take the apology as a genuine one:

"It's internal, it is in them, if they think that by offering compensation they have solved the problem and then they are just offering or making an apology just for the heck of it. That's the ... of the person already, maybe he is not really after forgiveness or reconciliation, he just wants to solve the problem with the intention of making another one in the future. So, it's very difficult to determine what's going on in a person's mind, but the we have indication through his action, that's the only thing, way we can probably at least closely guess what he is thinking by looking at what he is doing but again it's not full prove. I don't think that it is $100 \%$ accurate, because it's you know I called it band aid remedy. So you just force a band aid on the sore part. You are not really curing what cause the soreness in the first place. So you hide, when you put band aid like when you have a cut you hide what's hurting. You cover what's hurting, this is what these people are doing, if they are really not after genuine apology, they are just placing band aid over trying to cover up their wrong doings" [P5].

The same Filipino participant offers also a solution to this dilemma, which is about truth telling: 
"You expose what you did. Yeah, tell everything, don't omit or don't add your story, because once everyone knows the truth the unedited truth, forgiveness is easy, it's very easy to forgive, if the person asking for forgiveness. It's really (..) asking for it (.) if you are going to ask for forgiveness, but you are also giving justification for what you did and you are not really asking for forgiveness, if you ask for forgiveness you must admit and tell everything" [P5].

The demand for truth and truth telling is discussed within the literature about truth commissions (Tutu, 2000) for forgiveness the intergroup level. For victims, truth telling is of utmost importance as it reveals the seriousness of the apology, is an indicator of trust, and is a substantial part of the healing process (Staub, 2000; Tutu, 2000). Truth telling was also perceived as part of the overall process of forgiveness by German participants, as the following excerpt referring to Bush's politics regarding Iraq shows:

"We admit completely honestly; this war has been a war for oil and not to establish uhm democracy on their continent. Honesty, absolute honesty" [G1].

The German participants thought about the apology issue relatively unilaterally at the individual level, which is in line with previous psychological research on intrapersonal aspects of forgiveness that has been mainly conducted in Western settings representing more individualistic cultures (Sandage et al., 2005). The salvation of the victim or the self from ill feelings was discussed as the predominant outcome of forgiveness, and does not necessarily depend on an apology. However, as previous literature suggests, the role of the apology and its function within the forgiveness process is dependent on the wish to continue the relationship at both sides (Worthington, 2005).

Among the interviewees there was a common understanding that a respected political representative could and should take on responsibility for making an apology. This representative has an important role, because he or she is promoting trustworthiness to others on behalf of the whole group or even a nation. 
The public apology or acknowledgment has therefore no value if the representative acting as the messenger is not respected, as one German participant describes:

"The more charismatic, the more respected a politician is, the more sense would make a public apology. That's really important for me. Honestly, if George Bush apologised today for what he has done in Iraq, then no one would believe him" [G1].

Furthermore, consistent with previous findings (Rata et al., 2008) another German participant pointed out that the acts of apology should be in accordance with verbal or written statements and subsequent actions:

"I think an apology is trustworthy, if the things said in the apology are implemented (...) if the collective stands behind the official statement, if they act accordingly" [G2].

However, some German participants had problems with the concept of an apology that precedes the forgiveness process at the intergroup level, and thought of it more interpersonally as the following excerpt displays:

"I mean, the state probably apologised already couple of times and I mean how many apologies need to be carried out by the state to be accepted (.)I mean what should the state do, should it, I don't know, should it build a castle for everyone or should it apologise to each and everyone via handshake?" [G5].

This German interviewee had problems with the notion of being held responsible for something that happened in the past without being an active agent for what had happened (Branscombe \& Doosje, 2004). They argued against being blamed for something that they were not being directly responsible for:

"Yes, this is really abstract, probably because everything (at this level) is related to a sense of that people are held collectively responsible, some sort of clan custody, if you can say that. A lot of people are judged regardless of their responsibility or guilt for the conflict. In regard of a 1 to 1 conflict situation, you are able to clearly determine, because you can exchange faster and really discuss everything, but regarding a group, that is, well, yeah, in a group there are too many, yeah, in a group there are too many hidden little details" [G2]. 
In this way, the German participants distanced themselves from holding collective responsibility for past transgressions. Furthermore, they were inclined to transfer responsibility to the governmental level with was also found in previous research (Dresler-Hawke \& Liu, 2006; Páez et al., 2006). The underlying motive of this behaviour could be to preserve a positive social identity (Turner, 1999). However, participants had difficulties to apply individual-level concept of forgiveness to the group-level.

Nevertheless, the trustworthiness of the apology source is of vital importance (Nadler \& Liviatan, 2006) and can be regarded as an antecedent within the forgiveness process. These results are in concordance with previous findings about the importance of honesty and genuine apologies (Zechmeister, Garcia, Romero, \& Vas, 2004). According to Nadler and Liviatan (2006) apologies that seem to originate from untrustworthy sources are taken as dishonest attempts to misuse others and get away without further consequences like retribution.

Among the Japanese interviewees the apology was seen as either a formal obligation by the offending party which has no further meaning, or as a kind of ritual that has to be done repeatedly to be perceived as sincere at the individual level. If the apology is a set phrase to indicate politeness, the aim is not to ultimately heal a broken relationship, but to have done what is socially expected and to make both parties be able to quickly forget what happened. If the apology is perceived as sincere, the offender will be forgiven, which means the offender is given a last chance to make amendments. If the offender fails, the offended will withdraw, avoid further confrontations or break-up the relationship.

Furthermore, at the intergroup level the Japanese participants were reluctant about the procedure of offering a public apology. They mostly stated that an apology will not help and is not necessary. They isolated the attitudes towards the 
wrong doing from the need for an apology. The improvement of relationships between countries or groups was perceived as a self-governing process where an apology has no place as following excerpts from two Japanese participants portray:

"I think apology itself is not so important, but to show attitude ... apology is not important" [J2]

and

"I think it's not necessary, because if Japanese government express their apology, even if they express their apologies, the situation will not change, but as the relationships in other domains change, the attitude between countries will change, so apologies are not necessary anymore" [J5].

Furthermore, among the Japanese interviewees there were strong objections regarding a demand for an apology that is issued by people who were not directly affected by the past harm done. From their point of view they have no right for such a demand:

"National or ethnic (...) first of all (.) if it is a past event, I have no right to ask for it (apology) (.) it happened and it's not (.) against me (.) ... why does it matter to me?" [J1].

One reason for this objection is that among the Japanese participants the demand for an apology for past atrocities was confused with a demand for an apology issued by an ordinary Japanese citizen and not by the government.

“... (.) the point is ... I am not the one (.) who are, who was hurt. So I just I have no right to (.) ask for I mean eh (.) I don't have any right to ask for (.) apology or I don't have the right to forgive" [J1].

The same Japanese respondent pointed out that there seems to be a misunderstanding among Japanese people of what is expected from them:

"The problem I see between ah Korea and Japan is that Koreans ah (.) ah asking for apology (.) and it, it makes sense to me but ah I mean also Chinese ... but ah I mean ordinary Japanese (.) think that they ... are asking apology from themselves (from Japanese people)... and not from the government... and ah I think ... that why should I (.) I mean 
apologise? I haven't, I don't even know any Koreans or Chinese" [JI].

Here again, similar to the German sample, the need to be positively distinct from the former perpetrators was very high and any act that would induce some sort of guilt feelings were rejected. This is echoed in the literature investigating the phenomena of group-based or collective guilt (Branscombe \& Doosje, 2004; Montada, Schmitt, \& Dalbert, 1986; Schmitt, Behner, Montada, Müller, \& MüllerFohrbrodt, 2000). Even among theorists, the idea that a person can be guilty for something they have not done themselves is controversial. Therefore, for protective reasons and to maintain a positive self-image, this Japanese participant placed the responsibility at the governmental level, clearly separated from the individual:
"Probably Japanese government not directly but indirectly involved, so government is a kind of incorporated person, so there is continuity. The government is responsible. And am I responsible? No, I don't think so... and I am responsible in the sense that I am not affecting the Japanese government as a voter or as citizen to make it behave in a more responsible manner" [JI].

Germans are often reminded of the atrocities during the Third Reich due to educational syllabi at school, memorials, museums, and through the media (Oliner, 2008; Shriver, 2005). Therefore, it is quite likely that today's Germans want to separate themselves from the atrocities committed during World War II, since the seeking of forgiveness would also imply an admission of collective guilt:

"But I am always torn (...) to apply the term 'forgiveness' in this context. For me it is more important to be on the qui vive, because nowadays there are generations living that eventually don't get the large scale adversity of the Holocaust and I just don't know whether a 18 year old Israeli has to forgive me as a German ... (...) this forgiving suggests collective guilt (...) that I don't want to take on, I have to honestly admit. I do not feel collectively guilty" [G1].

All participants, however, agreed that the offender should be the initiator of the issue of an apology, regardless of at which level (individual vs. group) the harm 
done occurred. The offer of a public apology, which goes beyond the typical claims for financial compensation, is perceived to be a prerequisite before the forgiveness process at the broader level can even start:

"It must be in sync with the efforts. It doesn't make sense when you apologise and (...) let systems continue that are unfair" [P4].

Among the Filipino interviewees the concern was also raised that a public apology can be a strategy to get approval for and follow-up a different agenda:

"Public apologies sometimes are used in making government policies in crafting laws and the real objective is not met. The same our understanding but a public apology sometimes the sincerity is not that (...) The process includes a different agenda and it is not only asking forgiveness but it involves politics, policies" [P1].

Having been maltreated during war, colonisation periods and so forth, one Filipino participant simply put it like this:

"That they (Japanese) are sincere in their apology they are also sincere in helping us ... not necessarily financially, but you know in times of crisis, not just financially, just for support, moral support. That would do ...showing that they care" [P3].

Therefore, receiving signs of care and moral support can help victimised groups feel acknowledged as human beings, capable of the same sufferings as the perpetrator group. This can be explained through the notion of infrahumanization, or simply recognizing the human being in the other group again (Tam et al., 2007). These acts may reduce the perception of the outgroup as inhumane.

This Filipino participant points out that not seeking forgiveness and acknowledging the harm done is a denial of the past misdeed:

" (.) not seeking forgiveness on the one hand is not recognizing that this person exists and that you have actually done something wrong to this person or to this group" [P4]. 
In sum, among the interviewees, an apology alone was not considered enough; the offender was also expected to make amendments in form of compensations. This is echoed in the literature (Wohl \& Branscombe, 2005). All participants from the three countries agreed that an apology is of the utmost importance, if a continuance of the relationship is desired or even thought of.

It seems that the desire for a continuance of the relationship is more complicated to determine at the intergroup level compared to the interpersonal level. At the group level, the continuance of the relationship may be desired if the formerly conflicting groups share physical space or political and economic interests. Therefore, particularly at the intergroup level the prerequisite of a public apology helps to re-build fractured relationships after conflicts. The obligation to acknowledge past harm lies in the hands of the former perpetrators. This gets difficult at the intergroup level for large-scale conflicts where direct perpetrators may not be alive anymore, or where one group were at some stage victims and at another stage perpetrators (for example the Northern Ireland conflict). Furthermore, at the individual as well as the intergroup level the participants indicated that an apology has to be in sync with the perpetrators' efforts to make amendments. In other words, an apology is worthless, if, for example, the offences continue or no changes follow. Additionally, participants suggested that the forgiveness process requires a dialogue, in which each party can have a say.

Even though among the Japanese interviewees an apology was perceived as something instrumental, it was still a means to amend relationships. At the intergroup level, the issue of an official statement that acknowledges a past harm done was simply considered to be not sufficient to engender forgiveness. These perspectives are echoed in the literature (Digeser, 2001; Minow, 1998; Tavuchis, 1991). 
Collective emotions and memories

The interviews supported previous research, which has identified emotions and memories as antecedents of the forgiveness process (e.g., Tam, et al., 2007; Cairns \& Roe, 2003). In the interpersonal conflict literature forgiveness clearly differentiated from forgetting (Enright \& Rique, 2000). However, in the interviews forgetting equalled selectively forgetting ill feelings connected to the incident, as following excerpt portrays:

"You forget what happened, but you remember the lesson. You learn from that experience ... selective forgetting, maybe you forget the feeling of hatred, because when you say forgiveness that, it, it, (.) cannot that you are either mad or angry or maybe disappointed or perhaps not satisfied. So if you take that out, those negative things, (.) you will remember the lesson that you learnt. That get rid of negative feelings and forgiveness would be easier for me (...)" [P5].

Statements from other participants also suggested that 'forgetting' in the forgiveness process referred to selectively forgetting negative feelings attached to the conflict. This, however, has not been differentiated in the literature yet. Nevertheless, the Filipino participants also agreed on that it is hard to forget when something bad happened in the past and that similar situations will trigger memories:

"Something that life's altering cannot easily be forgotten and it's the truth that you do not forget these things because I don't know ... you wouldn't want to find yourself again similarly situated, you become aware of your company and things that are happening to you (...) and then (...) it's difficult to forget about something, when you forgive an offence (.) sometimes you do have forgiven that person and yet when you have similar encounter then it starts teasing back again, then you remember all of it (.) that's like keeping a score card (...) they just get logged there, this happened at this time, this happened at this time you might temporarily not think about it, but I am sure that a similar situation will bring back memories" [P4]. 
The German participants generally expressed similar views about forgetting, but they put it on the intergroup level. The following excerpt by one German interviewee highlights these perceptions:

"Uhm (...) you cannot basically forget such things, I have a hard time to believe this (.) ... there are so many different historical examples. Uhm, they will never forget this. Even every generation will never forget this ... basically I think, because (.) how would you be able to prevent things from happening again ... like with the Nazis in Germany or in other countries. There was this one wonderful example for this and you better not forget this and should do anything to keep it in your memory and that's why it is stupid to forget such things" [G2].

For the German participants who were perpetrators in the past, forgiveness at the group level had something to with not being confronted or reminded of the past misdeeds all the time. There is a tendency among the current public opinion in Germany to reject collective guilt (Dresler-Hawke \& Liu, 2006; Páez et al., 2006).

The following excerpt describes this opinion:

“... Germany, National Socialism or anti-Semitism, xenophobia (...) there is nothing to excuse really, uhm, you cannot really; first of all you cannot really forget it. You are able to forgive insofar by saying in principle the new generation in this country should not be linked to this anymore ... this happens to you again and again when travelling to a foreign country (...) ... (...) well, in principle (.) if I travel to a foreign country or when foreigners travel here to Germany, that these people do not permanently confront me with these things, for example, permanently being asked these questions or having to deal with my nation's past, because I belong to a different generation, but I deal with it for all I care, if in any discussions or in any conversations this topic is dropped, because it is a global topic by now. It is not restricted to Germany only... (...) but predominantly I think it is the thing that you are not permanently confronted with it. This the thing people forgive, I think, but also that it is no longer a topic in people's minds (.) and that are openly received and with complete distance (...) " [G2].

Generally speaking, memories are important in order to respect the victims and acknowledge how they were affected by the harm committed (Minow, 1998). 
However, among the German participants and Japanese participants, younger generations found it unpleasant to be linked with atrocities committed in the past and being confronted with it (Shriver, 2005). Memories, especially on a broader, collective scale that denote shared meaning systems within a country or specific group, help ensure that history does not repeat itself and give victims an avenue to commemorate and grieve appropriately (Páez \& Liu, in press).

However, the Japanese participants preferred the strategy to forget, if there was a conflict or argument at the individual level:

“Shoo (...) I can't remember (.) probably I (.) don't (.) want to remember those occasions. There is no use of remembering (.) such ah (.) events" [J1].

And

"For Japanese young people...I am not sure ... it's not only young ... we see, keeping in mind the original conflict or tiny problems is not smart, so we try to forget that ...not forgive that, we don't need to forgive, because we forget about the things about the conflict" [J2].

However, all these remarks refer to the interpersonal level of forgiveness. If the Japanese participants referred to the intergroup level, they isolated Japanese individuals from the governmental body, giving the government the responsibility to resolve outstanding disputes in a similar manner to the German participants. The reluctance of the Japanese participants to feel personally responsible for something that happened in the past is similar to the German participants' reluctance that is in line with previous research (e.g. Branscombe \& Doosje, 2004). The following extract from a Japanese participant displays this:

“(...) It's hard to say but I think everyone cannot be responsible for past things from very long ago because if one try to take responsibility for past things I don't know what extent we go back from so ... But government can take responsibility for example a formal investment or (...) it's not responsibility but cooperation $\ldots$... compensation is not possible but we can create another 
relationship so once a good relationship is created the past is not a problem I think" [J3].

There were some similarities among the Japanese and German interviewees in terms of the rejection of being held collectively responsible and being condemned as collectively guilty as perpetrators of past misdeeds. However, there were also some differences regarding the connotations of apologies at the intergroup level related to World War II. Previous research (Conrad, 2003; Hein \& Selden, 2000; Shriver, 2005) has indicated that Germans are generally more aware of the past misdeeds during World War II than Japanese.

Representation and historical closure with the past

How the past is constructed and passed on to the younger generations is a subject of research in sociology, political science and philosophy (e.g. Ignatieff, 1996; Ricoeur, 2004). It is hardly researched in psychology with some exceptions (e.g. Liu \& Hilton, 2005; Páez \& Liu, in press; Pennebaker et al., 1997). It is noteworthy that the past indeed can shape the future and promote a particular agenda (Liu et al., 2005) and Ignatieff (1996) concurred that the tragic matter about the past is that it does not belong to the past, making it 'poisonous', since it can fuel conflicts.

In the interviews, it was especially interesting how the past was represented by the German and Japanese participants, as these nations perpetrated crimes against humanity during World War II. When these participants spoke about the past, they mostly saw the whole matter from a very distant position due to the absence of guilt feelings related to past misdeeds. This is a common response for perpetrators (Páez, Marques, Valencia, \& Vincze, 2006) that probably had protective reasons as following extract shows:

"I mean, grandchildren and great-grandchildren and greatgreat-grandchildren, I think, they have never gotten this, 
they have hardly read something about this, all of them read somehow comics from the USA or some other fancy magazines (.), but they don't learn this anymore nowadays, but you don't (.) I believe a 14 year old or 10 year old child today don't need to repent (.), because people in the Third Reich did ..." [G5].

Furthermore, another German interviewee expressed it like the following extract shows:

"I am a disputatious person. I would assess myself as moderately left-wing and I am someone who always tries to do something against any form of racism, if it occurs, but this forgiveness related to the Holocaust is a discussion that I don't find contemporary anymore" [G1].

This extract also blends in what I would refer to as some kind of historical or psychological closure with past, since there seems to be no need any more to discuss it. There is probably this kind of "departure from the past" which Staub and Bar-Tal (2003) are referring to as symbolic means of healing also being symbolically related to forgiveness.

In order to maintain a positive image the Japanese participants portrayed the protest from other East Asian countries regarding Japan's handling of the past deeds during World War II as propaganda driven by the foreign government, questioning the trustworthiness of the sources. The extract by a Japanese participant portrays this opinion:

“(...) Regarding ... the ... problem I guess I don't know but I guess most of the Japanese people think the anger from China and Korea is a political one so maybe most of the Japanese people think most of the people in China and Korea (...) ... (...). Yeah as for China most of Japanese people don't think Chinese people is (are) feeling anger about (...) problem. Maybe it's a kind of political manipulation of information" [J4]. 
Similar to the German participants, this Japanese participant explained the matter as a problem for the older generation and not as problematic for the younger generations.

“(...) It depends on the generation. Maybe old people feel angry to us but young people not (...) so many young people don't feel angry to us. But in Korea maybe even young people feel angry but it's related to the current situation and including football so it's not about World War II" [J5].

It was noteworthy that the Japanese participants did not seem to care about any acknowledgements of any harm committed in the past to them (e.g. the Hiroshima bombing). This could be because a large-scale apology directed to them could have implications for being obligated to do the same:

"Some people said American governments have to apologise to Nagasaki or Hiroshima people ... I agree for most of it but if American government have to apologise to Japanese we Japanese have to apologise to more and more other Chinese and ...." [J2].

The concept losing face to one's people is a well-known phenomenon in East Asian cultures, since it signifies that the ancestors are respected. Japanese thus experience a dilemma about how they can be respectful towards their ancestors while at the same time not offending people whose ancestors suffered from Japanese in the past. However, things at the political level are perceived to be more strategic. A Japanese interviewee indicated that the actions - such as paying respect to Japanese soldiers who died during the war by visiting a shrine - carried out by the Japanese Prime Minister are offensive acts towards other East Asian nations, but strategic in order to gain approval from the Japanese people and win elections:

"Abe's ${ }^{8}$ grandfather is I don't know how to say (.) crime (war criminal) ... yeah and Abe maybe is proud of his grandfather ... But Koizumi is more strategical (...) he wants to win election so he wants to make the relatives of

\footnotetext{
${ }^{8}$ Abe Koizumi was the prime minister in Japan from 2001-2006.
} 
those people vote for him ... I can only imagine but if I was a relative of that people I am very proud of my grandfather I hear Koizumi has respect for my relatives (.) so it's (.) but I'm not sure I can only imagine" [J2].

Furthermore, among the Japanese participants there was a common understanding that Japanese government initiatives to resolve the past are potentially misused:

"Japanese initiative would be taken advantage of politically by Korean and Chinese government so that is at the core of the problem" [J5].

How the past is transmitted to the next generations plays a vital role to foster understanding of the roots of a conflict (Staub, 2000). Participants suggested that it should be a part of the educational syllabi, because otherwise the younger generations who continue to argue about a conflict that originated long time ago do not have an understanding about the causes, as following Filipino participant highlights:

"... if you are going to move in the, on the international scale and think about conflict, each old conflict before we were born, I mean this younger generations they do not even know how this conflict started and yet they are continuing it and I think that is how their children would do it also and how can you end it? (...) So somebody, someone has to make the conscious decision to really get to the bottom of things, what started it, how can we stop this thing. If those kind of mentality will not be introduced and I don't think that you can introduce that to the adults, you have to start with the children to solve this regional or ethnic conflict that we have right now in the world (...) ...before you forgive you must first be able to understand what's happening, what's caused it (...)" [P5].

Severity of harm done

The severity of the harm done has an influence on whether the victim will be ready to engage in a process of forgiveness and how an apology may be interpreted (Zechmeister et al, 2004). The more severe the harm done, the less 
likely the victim will be willing or will be ready to forgive (McCullough et al., 2000) as following extract by a Filipino interviewee describes:

"Sometimes it depends on the gravity if you want to just forgive ... but there is always this feeling afterwards that there is not always $100 \%$ in some instances especially when the gravity has cost you in your person. If it is too much usually I will forgive you, but sometimes it takes some time to heal" [P1].

The interviewees used every day experiences to describe how they felt about forgiveness. Due to the interview context it is understandable that the participants did not disclose very severe or painful experiences. However, they pointed out that many conditions they described only apply to minor conflicts as this Filipino participant explains:

"I am talking only about minor things, but I can imagine that for people who must have gone through very difficult experiences find it really hard to forgive" [P4].

Another Filipino participant pointed out that it also depends on the intentionality of the harm committed. If the transgression was committed accidentally, it is easier to engage in a process of forgiveness.

"I think that would influence my feelings also knowing that somebody did something bad to me on purpose (.) but if I know that it wasn't done on purpose it was accidental or without any fault then I think it would be easier also for me to forgive" [P1].

These perspectives are echoed among the German participants. The time factor was also mentioned, supporting previous research (e.g. McCullough, Fincham, \& Tsang, 2003) indicating that healing wounds takes time and healing deep wounds even more. Deep wounds inflicted at the group level and inherited in form of narratives to the younger generations probably will not fade with time. The following excerpt from this German participant portrays the time factor in relation to the severity of an offence: 
"Well, when something really bad happened that you want to forgive, then you definitely need more time. I can imagine, the more severe the offence is that you want to forgive, the longer it can take" [G4].

The extract below shows that a very severe incident can result in the rejection of any apologies from the perpetrator and in a break-up of the relationship:

"When something really, really rough happened, then I believe even an apology won't help anymore, well, when it was too severe (...) then there won't be forgiveness (...) thus, an apology won't make any sense, then it will be only a flowery phrase" [G5].

Therefore, willingness to engage in the forgiveness process depends on the context of the transgression and on the level of the perceived sincerity of an apology. This perspective is along the lines of the moral development model of forgiveness proposed by Enright and the Human Development Group (1998). Enright and the Human Development Group call this stage 'conditional forgiveness', because granting forgiveness is dependent on conditions or prerequisites the perpetrator has to meet.

However, this matter gets very complicated at the intergroup level. At the intergroup level the severity of the offence is likely to be already high due to the nature of the offence (e.g. ranging from social exclusion and denial of human rights to mass killings or the most severe form of genocide). Therefore, it may be understandable that forgiveness at this level is bound to specific conditions and prerequisites that have to be met in order to protect the victims. Furthermore, for neighbouring countries or groups within a country, the interpersonal-level option of breaking up the relationship may be impossible.

In summary, three themes that influenced forgiveness beyond the apology dealt with collective emotions, memories and how participants handled their own interpretation and perception of the past. An apology signals an acknowledgement 
that harm was done (Lazare, 2004). It also signifies to the victims that the perpetrators have a sense of guilt and an awareness of the effects on the victims. At the intergroup level, this theme was further explored through additional questions. According to the interviewees an apology seemed to be quite important to foster forgiveness and to heal a broken relationship. Representations of the past in particular allowed participants to distance themselves from a heavily burdened past in which their ancestors were perpetrators. The interviews highlighted that the perception of the past and historical closure may play a vital role in the forgiveness process, especially at the intergroup level. This however has not been well researched in relation to forgiveness. Hamber and Wilson (2003) dealt with symbolic closure in the context of the South African Truth and Reconciliation Commissions, but focused more on how this institution tried to force closure upon victims. In general, apology, collective emotions, memories, and severity of the harm done have been investigated in the literature at the individual level and at the group level (e.g. Branscombe \& Doosje, 2004; Cairns \& Roe, 2003; Worthington, 2005).

\section{Outcomes of Forgiveness}

What comes after forgiveness is achieved? This question was not a question on the interview schedule, but the participants did talk about potential outcomes of forgiveness. Outcomes of the forgiveness process at the intergroup are hardly discussed in the literature, but the literature that does exist argues for reconciliation as an outcome (see Noor et al., 2008). The literature on outcomes at the interpersonal level is related to intrapersonal variables like well-being due to the application of forgiveness within therapeutic settings (see Chapter 2). These intrapersonal outcomes of forgiveness were especially mentioned among the Filipino and German interviewees. 
Nevertheless, the consequences of the forgiveness process the participants talked about may have different implications at different levels of observation (interpersonal vs. intergroup). If forgiveness is achieved, the most typically mentioned positive outcome at both levels was reconciliation, which is in line with the few studies focusing on outcomes (Noor et al., 2008). In general, reconciliation was regarded as the end and forgiveness as the means. This, however, applied only for the participants from the Philippines and Germany. As the concept of forgiveness had somewhat negative connotations within the Japanese sample, reconciliation as an outcome (or any other outcomes) were not mentioned at all.

In line with previous research (e.g. Staub et al., 2003), participants suggested that forgiveness enables reconciliation through facilitating communication between the two parties, which may break the cycles of conflict. One Filipino participant describes it as follows:

"If there is forgiveness it would open up communications from both sides. If you don't forgive there will be a continuous cycle of violence and they will always retaliate for everything that is done and the hate will increase" [P1].

Breaking the cycle of violence was also mentioned by this Filipino participant as an outcome of forgiveness. It was noted that forgiveness allows the parties to move forward without getting into a vicious cycle of getting even:

"I think it's important. Also I think for me it's important because it's like breaking a cycle of violence. It has to stop someday and if that cycle isn't broken I don't think we would be able to move forward. ... without forgiving I think people would always think about revenge or getting back at someone (...)" [P1].

This Filipino participant also described the driving force of forgiveness as leading towards reconciliation; and considered the destructive role of anger that originated in the past and is carried on into the present: 
"I mean looking at what's happening right now I mean just right now in our country, I mean there are a lot of um bombings I mean and you know you think about it and you think why the this is happening, why they keep killing, I mean, they are also Filipinos why do they kill Filipinos, too. It's because they don't have reconciliation, they don't have forgiveness, they don't forgive you know the people who have [] have hurt them in the past and now they're doing it to those innocent people now I mean because they are still angry (...)" [P3].

The achievement of forgiveness was described as the starting point from which reconciliation is possible and can endure, such as by this German participant:

“... my opinion is that a lasting reconciliation process, an honest reconciliation process can only function, if forgiveness takes place first (.) I know historically viewed there are a lot of reconciliation processes, but I don't see them as lasting and I view them as half-hearted (...)" [G1].

Therefore, forgiveness is seen at least among the interviewees from the Philippines and Germany as a necessary step towards reconciliation. Therefore, reconciliation is seen as a function of seeking and granting forgiveness. This finding is not in accordance with findings about indigenous conceptions of forgiveness, where forgiveness was seen as an outcome which is preceded by a reconciliation process (Rata et al., 2008).

\section{Conclusion}

The "facets of intergroup forgiveness" theme captures the distinctions between interpersonal and intergroup forgiveness, the perspectives, understanding, meaning and associated benefits as well as costs of forgiveness in general. The core of the process is the "antecedents of forgiveness", which includes apologies, collective emotions, memories, the historical closure and representation of the past and severity of the harm. The "outcomes of forgiveness" theme displays what outcome is desired or anticipated. Outcomes were not mentioned as frequently as 
the elements of the other two themes, since outcome questions were not asked and were not part of the interview schedule. However, since outcomes were mentioned, the low frequency may be due to the intricacy of the concept "forgiveness" that is already complex at the interpersonal level, but has even more degrees of complexity at the intergroup level. Therefore, participants for the most part focussed on the antecedents and what forgiveness is at all. These themes are mapped in Figure 4.1.

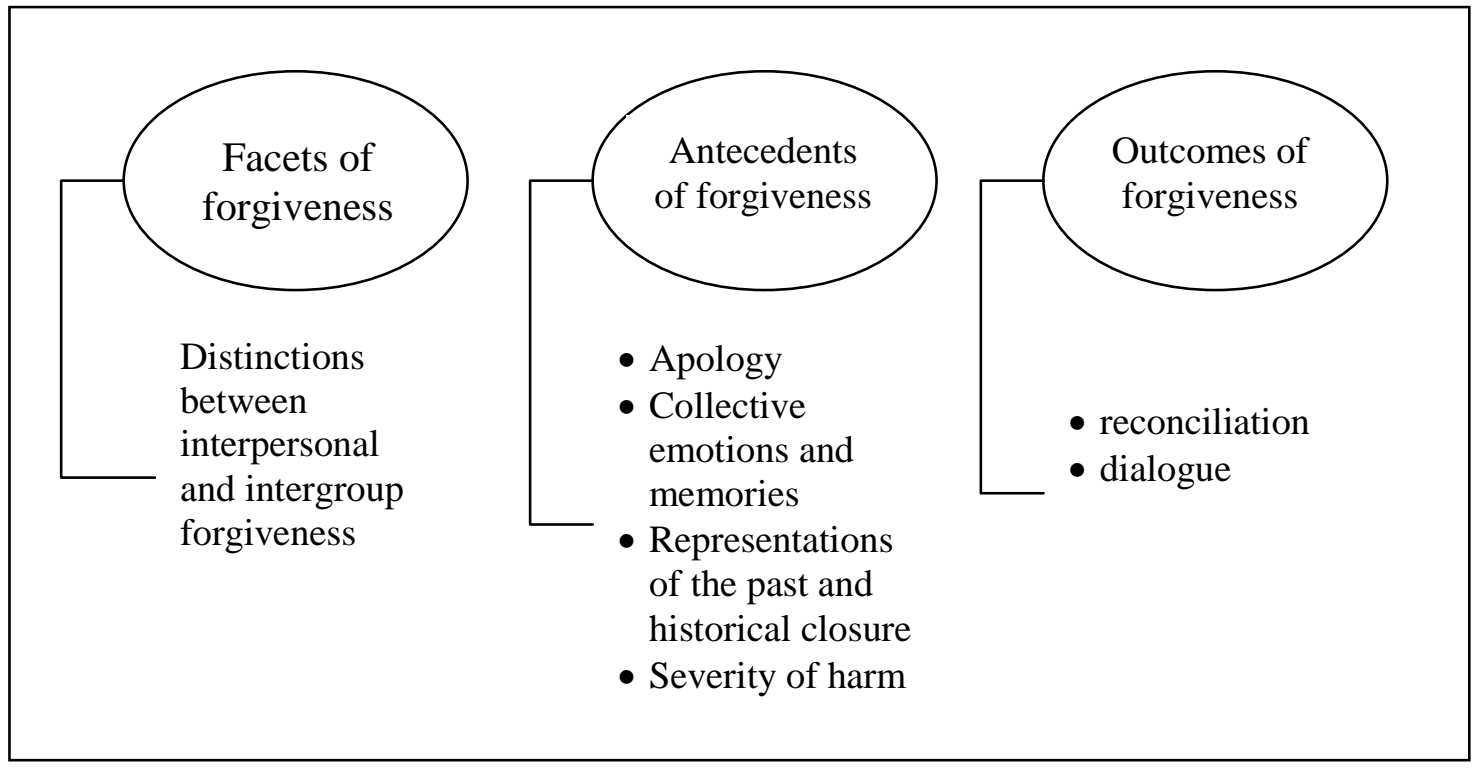

Figure 4.1 Summary of identified themes (Study 2)

Among the German and Filipino interviewees' general perception of forgiveness was positive. Therefore, at the onset of the forgiveness process, forgiveness was perceived as a meaningful and an important means for conflict resolution at both levels (interpersonal and intergroup). Perceived hindrances in the forgiveness process were different representation of the past, closure or lack of closure with the past, the level of guilt feelings (perpetrators), and the level of guilt assignment (victims). It seems that this process might be circular, meaning that it can go through cycles in order to achieve sustainable reconciliation. However, this would be a matter for longitudinal testing. 
Among the Japanese forgiveness was perceived as marginal for conflict resolution and was only considered if it was perceived as useful for a particular purpose. Therefore, for the Japanese participants forgiveness was not about letting go of anger or release from ill feelings attached to the conflict, but rather as a process which could offer a utilitarian benefit such as a more committed relationship or repetitive apologies to stress relational sincerity. It seems that this view on conflict resolution at the intergroup level is highly related to how the Japanese participants perceive the past and consequently the relevance of forgiveness. As they considered apologies at the intergroup level irrelevant, whereas the German and Filipino participants perceived apologies to be a crucial conditional component, it is understandable that attempts of rapprochement, if made, between former victimized East Asian nations and Japan are working from a different set of meanings fraught with greater difficulty.

Compared to the ease of discussing forgiveness at the individual level, the participants seemed to struggle with what intergroup forgiveness is and how forgiveness can be applied at this level. The participants argued that forgiveness at the individual level is not as complex as at the group level. Moreover, the participants mentioned that forgiveness between two people is easier to achieve than between groups which is echoed in the literature (e.g. Hewstone et al., 2004).

However, the qualitative data suggested a distinction between interpersonal and intergroup forgiveness where different processes might occur. Interpersonal forgiveness seems to exist as a distinct and concrete concept whereas intergroup forgiveness does not. Therefore, the interviewees had difficulties in articulating what intergroup forgiveness is. Nevertheless, the interviewees were able to talk about the differences between interpersonal and intergroup forgiveness. Participants identified several discrete processes involved in intergroup 
forgiveness. These discrete processes belonging to the intergroup forgiveness process were for instance how a public apology should be issued (in line with the democratic process deemed as important by Kadima Kadiangandu \& Mullet, 2007; Kadima Kadiangandu, Gauché, Vinsonneau, \& Mullet, 2007) and whether the historical past is shared and seen as relevant or irrelevant for the future. The mentioned process elements are different from interpersonal forgiveness.

Therefore, the extracts portray a whole forgiveness process, with overlapping perceptions about aspects of this process across the different cultural backgrounds. Overall, the findings suggest a clear distinction between interpersonal and intergroup forgiveness. Forgiveness at the individual level was for most participants limited to two people involved directly in a conflict. Consistent with the literature (Enright \& the Human Development Group, 1991, 1998), for most Filipinos and German interviewees being forgiving at the individual level is beneficial for the self (e.g. "stress reliever"), whereas being unforgiving is associated with costs (e.g. "energy consuming, unfair to yourself”). However, for the Japanese participants forgiveness as such only seemed to have a utilitarian value if it allowed them to receive something in exchange.

I may cautiously suggest a cross-cultural difference between the Japanese and the other interviewees (Filipino and German) with respect to their conceptions about forgiveness. The Japanese participants mostly talked about conflict avoidance as a means of handling conflicts. Most of the Japanese participants favoured the option of suppressing their memories about offences against them. In addition, when they talked about forgiveness, they mostly spoke about conditional aspects like an apology being a necessity in order to be able to forgive. As they discussed forgetting about an offence, they also mentioned that they were suppressing their emotions, because it was shameful to express emotions linked to 
conflicts. This is consistent with the model of maintaining superficial harmony in more collectivistic cultures such as Japan or among Chinese (Brew, 2007; Huang, Jone, \& Peng, 2007), and preferences for conflict resolution strategies such as withdrawal and avoidance over forgiveness (Ohbuchi et al., 1994).

Furthermore, forgiveness at the intergroup level has to go through stages and therefore, can be described as a process. When the participants spoke about intergroup forgiveness, they often made reference to the constitution of a group, which seemed to complicate the whole matter of forgiving. The findings suggest a specific way to draw the line between interpersonal and intergroup forgiveness. Whilst forgiveness at the individual level is perceived as a unilateral decision, which benefits the self for the most part, more external factors come into play at the group level, and the benefits to an individual are less obvious. External factors are contextual factors, such as the representation of the past and the dealing with past by governmental or representative institutions.

Participants talked about leaders and group representatives bearing responsibility to open up communication between the group they represent and the other party. This implies that intergroup forgiveness is a based on an interactive exchange between the victim and perpetrator. The participants talked about institutional factors that might have an impact on forgiveness at the intergroup level (such as representatives apologizing on behalf). This suggests that a lot more external factors have an impact on forgiveness at the intergroup level, so that an individual would find it hard to unilaterally forgive an intergroup level grievance. Furthermore, cultural context may have an impact on views of forgiveness as this perspective was clearly articulated in the German and Filipino interviews compared to a weak expression in the Japanese interviews where forgiveness was neither 
considered to be a powerful means to resolve interpersonal conflicts nor in intergroup conflicts.

The representation of the past transmitted through public and politicized opinions may have an impact on the reasoning about forgiveness at the intergroup level which is in line with social representations theory (Moscovici, 1984) and social representation of history (Liu \& Hilton, 2005). The historical background plays a vital role for the perception of the need of forgiveness - especially with regards to whether history is perceived as still having an influence on current relationships, rather than as part of the past. Since this discovery has not been explored elsewhere in the literature, I will pay particular attention to this aspect in the subsequent studies, and attempt to investigate 'closure with the past' by assessing it. 


\section{Chapter 5}

\section{Study 3: Victim perspectives - Intergroup forgiveness across European and East Asian contexts}

Study 3 is divided into two parts. The first part (Study 3a) deals with differences between six societies and how the new concepts (historical closure, costs of granting forgiveness) relate to established concepts (such as collective guilt assignment). The second part (Study 3b) focuses on the unique contribution of the new concepts over and above established concepts to predict intergroup forgiveness across two contexts (Europe vs. East Asia). Furthermore, it examines to what extent context relevant variables explain variance in intergroup forgiveness using a cross-level operator analysis.

\section{Introduction and outline of Study 3a}

As Cairns and colleagues (2005) noted, more research is needed that taps into different stages of a conflict in order to determine where forgiveness processes are most helpful and to foster reconciliation processes. Manzi and Gonzáles (2007) additionally noted that the intergroup forgiveness process is still poorly understood. Therefore, the main objective of this study is to extend the research conducted in this area and to shed more light on the process in the post WW II context. I gathered data from two post WW II contexts that included six formerly victimized societies; three from Europe (France, Poland, and Russia) and three from East Asia (China, Philippines, and Taiwan).

Central aims of this study were to investigate the psychological correlates (collective guilt assignment, group-based anger, outgroup trust, and the new variables historical closure and perceived costs of granting forgiveness) of and differences in intergroup forgiveness across these six societies. As already 
described in Chapter 2, the post WW II context is a very meaningful natural laboratory, for Germany and Japan have adopted different strategies over the course of the past half-century for handling their WW II past. This should have definite consequences in terms of their neighbours' willingness to forgive them for past misdeeds.

Due to the different social representations of WW II and its aftermath facilitated through political elites, it is likely that the willingness to forgive will be different across our six participating societies. Specifically, it is quite likely that Chinese participants will be less forgiving compared to Taiwanese and Filipino participants due to the political and historical context (see Liu \& Atsumi, 2008), and that the French participants will be most forgiving due to early and sustained Franco-German rapprochement politics after WW II compared to Polish and Russian participants (Feldman, 1999). Therefore, I hypothesize that due to the societal context:

Hypothesis 1 ( $\mathrm{H}_{1}$ societal context hypothesis): Intergroup forgiveness will differ significantly across the six societies. Chinese participants should score lower on forgiveness compared to Taiwanese and Filipino participants, whereas French participants should score higher on forgiveness compared to Polish and Russian participants.

\section{Relationships between variables}

Historical closure and costs of forgiveness

Historical closure is conceptualized as an attitudinal content drawn from the historical context. It influences the extent that the past is perceived to still affect the present and future, and implications it has for present-day relationships between 
groups. Therefore, historical closure can be understood as an attitude towards the past and its relevance to the present and future.

It is plausible that non-closure with respect to the past will affect the propensity to forgive the other group. If past misdeeds are perceived as a vivid part of the present, there is no closure, and this lack of closure may prevent forgiveness. If the past is perceived as a closed book, people do not have to worry about whether it can or will have further negative influences. Therefore, in such cases it may be easier to show forgiveness to the former perpetrator. Having closure with respect to the past is also related to a "psychological departure from the past" (Staub \& Bar-Tal, 2003, p. 733), which can become a symbol for forgiveness and for new peaceful relations (Bar-Tal \& Teichman, 2005). Thus, there should be a positive association between historical closure and intergroup forgiveness.

Additionally, one topic that has been largely neglected in interpersonal and intergroup forgiveness research is the investigation of the potential costs of granting forgiveness (see McCullough, Pargament, \& Thoresen, 2000). To the best of my knowledge, qualitative work by Rata, Liu, and Hanke (2009) is the first research addressing this variable at the intergroup level. A lot of research has emphasized the potential benefits of interpersonal forgiveness for mental and physical health (for an overview see McCullough et al., 2000; Worthington, 2005), but perceived costs of forgiveness are especially important in unresolved disputes between groups (see Rata et al., 2009), where forgiveness might be construed as condoning injustice. Potential costs of forgiveness have been theorized (Baumeister, Exline, \& Sommer, 1998), but empirical investigation has remained at the interpersonal level and is sparse (Exline, Worthington, Hill, \& McCullough, 2003). 
Costs of forgiveness discourage people to engage in forgiveness. Especially in unresolved disputes, potential costs associated with granting forgiveness include the possibility that forgiveness leaves victims feeling powerless. How can forgiveness proceed if the perceived costs outweigh the potential benefits-for instance in a "post"-colonial context where the victimizers retain the benefits of their colonial era actions, and the victims still bear the costs (see Rata et al., 2009)? I attempt to address a general gap in the literature by investigating the perceived costs of granting forgiveness and its influences on the forgiveness process at the intergroup level. McCullough (2008) noted that when costs overshadow the benefits of forgiveness, the likelihood is quite high that an alternative is favoured that is negatively related to forgiveness (e.g. revenge, withdrawal or staying resentful). Moreover, he noted that forgiveness and its alternatives are contextsensitive. Hence, the costs of granting forgiveness should be negatively associated with intergroup forgiveness. Historical closure and costs of forgiveness should be negatively associated.

\section{Collective guilt and anger}

As outlined in Chapter 2, collective guilt assignment (see Wohl \& Branscombe, 2005) and anger (Mackie \& Smith, 2002) are potentially linked to non-closure with the past since the degree of assignment of guilt and anger are probably based on "open accounts" and open disputes. That is, the likelihood of assigning guilt to and feeling anger towards the perpetrator party is quite high and a departure from the past is difficult, if there are more unresolved disputes in post conflict settings.

For example, each Wednesday former Korean Comfort Women protest in front of the Japanese embassy in their quest for an official apology and compensation. Their lack of success elicits more group-based anger among many 
Koreans, not just the former comfort women (Chai, 1993; Coday, 1998; Cohen, 2006; Hicks, 1999). Similar protests are also organized in the Philippines due to the Comfort Women issues (Mendoza, 2003) and in China due to unresolved issues regarding the Nanjing massacre (Chan \& Bridges, 2006; Cody, 2005; Kim, 2001). Collective guilt assignment and feelings of anger are more likely in the context of an unresolved conflict, so these should be less pronounced where the FrancoGerman relationship is concerned (Feldman, 1999). In accordance with previous research, collective guilt assignment and forgiveness should be negatively related. Furthermore, collective guilt assignment and group-based anger should be negatively related to historical closure and positively related to costs of forgiveness. In general, it has been argued that forgiveness is predominately an emotional construct (see Tam et al., 2007) and it can be argued that its relationship to group-based emotions such as guilt and anger should be close to forgiveness.

\section{Trust}

Lack of trust is one of the key issues in long-lasting conflicts. According to Nadler and Liviatan (2006) lack of trust is a typical response to unresolved conflicts and one of the key obstacles to reconciliation. It usually entails the belief that the outgroup has bad intentions regarding the ingroup (Mitchell, 2000; Noor et al., 2008). Previous research has demonstrated that trust positively contributes to the process of forgiveness at the intergroup level (e.g. Noor et al., 2008). The degree of trust, however, may vary according to the context. Formerly victimized groups are likely to be suspicious of the agendas that drive the actions of former perpetrator groups, even if ostensibly harmless, when past conflict is unresolved. The restoration of trust is crucial, for it advances benevolence towards and reduces mistrust of the outgroup (Lewicki \& Wiehoff, 2000). As a positive link between trust and forgiveness has already been established by previous research (e.g. Noor 
et al., 2008), outgroup trust should be positively linked to historical closure and negatively to costs of forgiveness.

The role of identification with one's national group.

Previous intergroup forgiveness research applied a social identity/social categorization framework (see Chapter 2, SIT; Tajfel \& Turner, 1986; SCT; Turner, 1987). It established links in line with SIT/SCT research where identity was examined as either a mediating variable (e.g. Noor et al., 2008) or a direct predictor (e.g. Cairns, Hewstone, Niens, \& Tam, 2005; Noor, Brown, González, Manzi, \& Lewis, 2008) of intergroup forgiveness. Noor and colleagues (2008) argue that intergroup forgiveness relates to concerns entailing identity. Drawing from SIT, Noor and colleagues further argue that this distinguishes interpersonal from intergroup forgiveness. They propose that strength of group identification is an underlying force driving the relationships between other variables and intergroup forgiveness. It can be argued along the same lines that the perception of the costs related to granting forgiveness and the attitudes towards the past (historical closure) have an impact on one's self-perception. That is, the perception of costs for granting forgiveness and lack of historical closure may be connected to a stronger identification with the victimized group.

National identity should be negatively related to historical closure and positively to costs of forgiveness. Furthermore, following SIT/SCT, national identity should mediate the relationship between (a) costs of granting forgiveness and intergroup forgiveness, and between (b) historical closure and intergroup forgiveness.

In summary, the hypotheses for this study can be divided into four sets of predictions. The first one is about societal context: 
Hypothesis $1\left(\mathrm{H}_{1}\right.$ - societal context hypothesis): Intergroup forgiveness will differ significantly across the six societies. Chinese participants should score lower on forgiveness compared to Taiwanese and Filipino participants, whereas French participants should score higher on forgiveness compared to Polish and Russian participants.

The second set is about positive relationships between variables:

Hypothesis $2\left(\mathrm{H}_{2}\right.$ - positive relationships): Historical closure should be positively associated with trust and intergroup forgiveness. Costs of granting forgiveness should be positively related to collective guilt assignment, group-based anger and national identity.

The third set concerns negative associations between variables:

Hypothesis $3\left(\mathrm{H}_{3}\right.$ - negative relationships): Historical closure should be negatively associated with costs of granting forgiveness, collective guilt assignment, groupbased anger and national identity. Costs of granting forgiveness should be negatively related to outgroup trust and intergroup forgiveness.

The last hypothesis deals with the role of social identification:

Hypothesis $4\left(\mathrm{H}_{4}-\right.$ mediation): Identification with one's national group should mediate the relationship between historical closure and intergroup forgiveness and between costs of granting of forgiveness and intergroup forgiveness.

In sum, I will examine the differences in intergroup forgiveness across the six societies $\left(\mathrm{H}_{1}\right)$ and will investigate the relationships between historical closure, the costs of forgiveness and established psychological variables $\left(\mathrm{H}_{2}\right.$ and $\left.\mathrm{H}_{3}\right)$ in order to investigate the nomological net of the two new concepts, and shed light on intergroup forgiveness in the setting of post WW II relations. $\mathrm{H}_{4}$ will test the role of identification with one's national group. 


\section{Method}

\section{Participants}

An anonymous questionnaire was administered to 1197 university students (mean age $=20.42, S D=3.28$, female $=62.2 \%, 0.2 \%$ did not disclose their gender $)$ in the Philippines $(\mathrm{N}=200$, Manila), China $(\mathrm{N}=202$, Zhuhai and Guangzhou), Taiwan (N=222, Taipei), France (N=206, Toulouse), Russia (N=200, Saratov) and Poland ( $\mathrm{N}=167$, Warsaw) (for an overview of sample characteristics see Table 5.1). All questionnaires were administered in the native language of each participating country, except for the Filipino sample where the questionnaire was administered in English, which is an official language in the Philippines. In most cases a translation-back-translation method was used to ensure correct translation of the questionnaire. For the Polish version of the questionnaire, a bilingual committee approach was employed (van de Vijver \& Leung, 1997).

Table 5.1

Sample description $(N=1197$, Study $3 a)$

\begin{tabular}{lcccc}
\hline \multirow{2}{*}{ Society } & \multirow{2}{*}{$\mathrm{N}$} & \multicolumn{2}{c}{ Gender } & Age \\
\cline { 3 - 4 } & & Female & Male & M (SD) \\
\hline Taiwan & 222 & $49.5 \%$ & $50.5 \%$ & $20.94(2.39)$ \\
Philippines & 200 & $47 \%$ & $53 \%$ & $20.48(5.2)$ \\
China & 200 & $29.7 \%$ & $69.3 \%$ & $19.42(2.08)$ \\
France & 206 & $82.5 \%$ & $17.5 \%$ & $21.81(4.24)$ \\
Poland & 167 & $85 \%$ & $15 \%$ & $20.78(1.28)$ \\
Russia & 200 & $78 \%$ & $22 \%$ & $19.06(1.07)$ \\
\hline Total & 1197 & $62.3 \%$ & $37.7 \%$ & $20.42(3.27)$ \\
\hline
\end{tabular}

\section{Instruments}

Participants completed a questionnaire assessing demographic information (e.g. age gender, birth country) at the end following self-report measures of interest (see Table 5.2 for descriptive statistics). Please see Appendices B1 and B2 for information sheet, questionnaires, and debriefing form for both contexts. 
Intergroup forgiveness. Intergroup forgiveness was assessed using three items based on Moeschberger and colleagues' scale (2005) and four items from Wohl and colleagues' scale (2005). The items were adapted to the World War II context regarding Japan and Germany. Sample item are "It is important that [your country] never forgives the past harm done during the occupation of Asia, the SinoJapanese War and World War II by Japan/ It is important that [your country] never forgives the past harm done during Hitler's rule and World War II by Germany" (reverse-scored) " and "Today's Japanese should be forgiven for what their group did to members your country] during Japan's period of colonisation and warfare/ Today's Germans should be forgiven for what their group did [members of your country] during Hitler's rule and World War II".

The overall scale proved to be reliable (overall alpha .80, Cronbach's alphas ranged from .66 to .84 across societies). Inter-item correlations indicated that the items are homogenous (mean inter-item correlation $=.37$, ranging from .22 to .44 across societies). Higher values denote greater forgiveness.

Historical closure. This scale was constructed for the study using items derived from qualitative interviews (see Chapter 4; Hanke, Liu, \& Fischer, 2008). The historical closure scale with six items assesses the degree to which the participants perceive the past as a part of the present and future, as opposed to being unconnected to the present/future.

Items were, for example, "Discussions about apologizing and forgiving related to Japan's harmful past actions against [members of participating society] are not important anymore/ Discussions about apologizing and forgiving related to Germany's past war crimes are not important any more" and "[Members of participating society] stories of surviving Japan's past harmful actions make me feel like the wounds are still open today/[Members of participating society] stories 
of surviving Germany's past war crimes make me feel like the wounds are still open today" (reversed-scored).

The overall alpha coefficient for the scale was .70; internal consistencies ranged from .60 to .75 across societies. The mean inter-item correlation was .27 , ranging from .19 to .33 across societies proving homogeneity of items. A higher value indicates greater closure with the past.

Perceived costs of granting forgiveness. A five item scale constructed for this study measured the perceived costs of granting forgiveness. Items were derived from qualitative work (Hanke, Liu, Fischer, \& Vauclair, 2007; Rata, Liu, \& Hanke, 2009) and the literature (Obuchi, 2007). Items were "Granting forgiveness will put the victimized group into an even more vulnerable position than before", "Granting forgiveness without empowering the victimized group can lead to a loss of identity for the victimized group", "Granting forgiveness is an invitation for crimes to be repeated", Granting forgiveness means losing sympathy as victims" and "Granting forgiveness will make the perpetrator believe that now everything is alright although nothing is really settled". The overall internal consistency was .75; Cronbach's alphas ranged from .68 to .80 . A higher value denotes greater perceptions of potential costs of forgiveness. Mean inter-item correlation was .37 ranging from .30 to .44 across societies indicating homogenous items. A higher value denotes more perceived costs of granting forgiveness in general.

Collective guilt assignment. Collective guilt assignment was assessed using a scale adapted from Branscombe and Doosje (2004). The five items measured the degree to which participants believe collective guilt should be designated to contemporary Germans and Japanese, in Europe and East Asia respectively. A sample item is “Today's Germans/Japanese people should feel regret for some things their ancestors did to [members of participating society] in the past." The 
overall internal consistency for this scale was .86; Cronbach's alphas ranged from .79 to .91 with a mean inter-item correlation of .56 (range: .43 to .66 across societies). High values indicate high assignment of collective guilt.

Group-based anger. Group-based anger was measured with four items adapted for this study from Mackie, Devos, and Smith (2000). Participants were asked to rate how much they felt each emotion (angry, displeased, furious, irritated) when thinking of the dealing with the past misdeeds by Germans or Japanese respectively on a 7 -point Likert scale $(1=$ not at all, $7=$ extremely $)$. Overall Cronbach's alpha was .89; internal consistencies ranged from .82 to .89 ; mean inter-item correlation $=.67$ ranging from .55 to .67 . A higher value captures greater anger towards Germans or Japanese due to their government's handling of the past misdeeds.

Outgroup trust. Outgroup trust was measured with a three item scale based on Brehm and Rahn (1997). I modified the scale to suit the European and Asian contexts (items: "Do you think today's Japanese people/Germans would try to be fair to [members of your society]?", "Would you say that most of the time today's Japanese people/Germans try to be helpful to [members of your society]?", “Generally speaking, would you say that most today's Japanese people/Germans can be trusted?"). A 7-point Likert scale was used ( $1=$ not at all, $7=$ very much). The overall alpha was .77 , Cronbach's alphas ranged from .56 to .84 across the six societies with a mean inter-item correlation of .52 ranging from .32 to 64 . A higher value indicates greater outgroup trust.

Identification with national group. National Identity was assessed using a shortened version of Leach and colleagues' (2008) scale. Twelve items were included to this measure. Participants stated their agreement on a 7-point Likert Scale ranging from 1 (strongly disagree) to 7 (strongly agree). Example items are 
"I feel a bond with [members of participating society]" and "[Members of participating society] have a lot in common with each other". The original alphas ranged from .80 to .93 (Leach et al, 2008). In this study, the overall alpha was .92; the internal consistencies ranged from .85 to .94 across the six societies; mean inter-item correlation was .48 (range from .32 to .58 across the societies). A higher value indicates greater identification with one's national group.

\section{Analytical Strategy}

Hypothesis 1 was tested using an ANOVA to assess the differences between societies. The relationship hypotheses $\left(\mathrm{H}_{2}\right.$ and $\left.\mathrm{H}_{3}\right)$ were examined through Pearson-Product-Moment correlations and multidimensional scaling (MDS) procedure. The correlations of predicted associations from each sample were then compared using similarity of correlation coefficients (Chi-square test of r-to-z transformed correlation coefficients). Hypothesis 4 was tested using the software MedGraph (Jose, 2003).

\section{Results}

\section{Descriptive statistics and reliabilities}

Table 5.2 presents the descriptive statistics and reliabilities for the instruments used for Study 3. 
Table 5.2

Means, Standard Deviation, Cronbach's alpha, F-values (society as fixed factor) (Study $3 a$ )

\begin{tabular}{|c|c|c|c|c|c|c|c|c|c|c|c|c|c|}
\hline \multirow{2}{*}{$\begin{array}{c}\text { Society } \\
\text { Scale }\end{array}$} & \multicolumn{2}{|c|}{ Taiwan } & \multicolumn{2}{|c|}{ Philippines } & \multicolumn{2}{|c|}{ China } & \multicolumn{2}{|c|}{ France } & \multicolumn{2}{|c|}{ Poland } & \multicolumn{2}{|c|}{ Russia } & \multirow[b]{2}{*}{$\mathrm{F}(5,1191)$} \\
\hline & $\begin{array}{c}\mathrm{M} \\
(\mathrm{SD})\end{array}$ & $\alpha$ & $\begin{array}{c}\mathrm{M} \\
(\mathrm{SD})\end{array}$ & $\alpha$ & $\begin{array}{c}\mathrm{M} \\
\text { (SD) } \\
\end{array}$ & $\alpha$ & $\begin{array}{c}\mathrm{M} \\
(\mathrm{SD})\end{array}$ & $\alpha$ & $\begin{array}{c}\mathrm{M} \\
(\mathrm{SD})\end{array}$ & $\alpha$ & $\begin{array}{c}\mathrm{M} \\
(\mathrm{SD})\end{array}$ & $\alpha$ & \\
\hline Forgiveness & $\begin{array}{l}4.62 \\
(.86)\end{array}$ & .80 & $\begin{array}{l}4.72 \\
(1.04)\end{array}$ & .80 & $\begin{array}{l}3.99 \\
(.89)\end{array}$ & .66 & $\begin{array}{l}5.45 \\
(1.12)\end{array}$ & .83 & $\begin{array}{l}5.10 \\
(1.04)\end{array}$ & .78 & $\begin{array}{l}4.75 \\
(1.27)\end{array}$ & .84 & $44.81 * * *$ \\
\hline Historical Closure & $\begin{array}{l}4.38 \\
(.88)\end{array}$ & .66 & $\begin{array}{l}4.17 \\
(1.09)\end{array}$ & .75 & $\begin{array}{l}2.90 \\
(.96)\end{array}$ & .62 & $\begin{array}{l}3.68 \\
(.97)\end{array}$ & .60 & $\begin{array}{l}3.60 \\
(.95)\end{array}$ & .62 & $\begin{array}{l}3.90 \\
(1.02)\end{array}$ & .60 & $58.21 * * *$ \\
\hline $\begin{array}{l}\text { Costs of } \\
\text { Forgiveness }\end{array}$ & $\begin{array}{l}3.12 \\
(.88)\end{array}$ & .68 & $\begin{array}{l}3.35 \\
(1.21)\end{array}$ & .80 & $\begin{array}{l}2.91 \\
(1.00)\end{array}$ & .70 & $\begin{array}{l}2.45 \\
(.92)\end{array}$ & .71 & $\begin{array}{l}2.53 \\
(.94)\end{array}$ & .74 & $\begin{array}{l}3.17 \\
(1.08)\end{array}$ & .71 & $25.34 * * *$ \\
\hline Guilt & $\begin{array}{l}4.28 \\
(1.16)\end{array}$ & .88 & $\begin{array}{l}4.24 \\
(1.50)\end{array}$ & .91 & $\begin{array}{l}5.09 \\
(1.16)\end{array}$ & .81 & $\begin{array}{l}3.04 \\
(1.21)\end{array}$ & .79 & $\begin{array}{l}4.00 \\
(1.37)\end{array}$ & .86 & $\begin{array}{l}3.92 \\
(1.28)\end{array}$ & .81 & $55.21 * * *$ \\
\hline Anger & $\begin{array}{l}3.77 \\
(1.42)\end{array}$ & .89 & $\begin{array}{l}3.93 \\
(1.44)\end{array}$ & .83 & $\begin{array}{l}4.40 \\
(1.26)\end{array}$ & .82 & $\begin{array}{l}1.85 \\
(1.02)\end{array}$ & .87 & $\begin{array}{l}2.64 \\
(1.31)\end{array}$ & .86 & $\begin{array}{l}3.40 \\
(1.54)\end{array}$ & .84 & $97.06 * * *$ \\
\hline Trust & $\begin{array}{l}4.13 \\
(1.08)\end{array}$ & .81 & $\begin{array}{l}3.92 \\
(1.22)\end{array}$ & .76 & $\begin{array}{l}3.59 \\
(1.25)\end{array}$ & .84 & $\begin{array}{l}5.00 \\
(.94)\end{array}$ & .56 & $\begin{array}{l}3.90 \\
(1.17)\end{array}$ & .81 & $\begin{array}{l}3.91 \\
(1.15)\end{array}$ & .63 & $36.75 * * *$ \\
\hline National Identity & $\begin{array}{l}5.22 \\
(1.02)\end{array}$ & .93 & $\begin{array}{l}5.52 \\
(.94)\end{array}$ & .89 & $\begin{array}{l}5.87 \\
(1.08)\end{array}$ & .94 & $\begin{array}{l}4.25 \\
(.88)\end{array}$ & .85 & $\begin{array}{l}4.74 \\
(1.06)\end{array}$ & .91 & $\begin{array}{l}5.30 \\
(.91)\end{array}$ & .85 & $68.73 * * *$ \\
\hline
\end{tabular}




\section{Distributional Normality}

According to Tabachnik and Fidell (2007) skewness and kurtosis indicate whether the data deviates from normality. Skew values larger than 2.0 and Kurtosis values greater than 7.0 point to problems with normality. There were no serious deviations from normality as the skew values were below 2.0 and the kurtosis values were below 7.0 for each item in each society.

\section{Structural Equivalence}

Multi-group confirmatory factor analysis (CFA) was used to assess the measurement model for the newly devised scales historical closure and costs of forgiveness in order to test for structural equivalence. Structural equivalence was tested since these scales are new and the underlying structure should be equivalent across all societies in order to justify comparisons. The other scales are established measure of well known constructs that have been widely used. Therefore, equivalence was not tested for these measures and I relied on the reliability of these measures. First, a single-group CFA was performed for each society separately as a prerequisite for testing factorial invariance (see fit indices for single group analyses in Appendix B3). Subsequently, the multi-group CFA was conducted yielding acceptable fit indices, indicating structural equivalence of these constructs across all six societies (see Table 5.3.). Because of the good fit indices, it is valid to assume that the content of all scales had the same underlying meaning across the six societies. It is, therefore, also justifiable to conduct comparisons. 
Table 5.3

Multi-group CFA fit indices for historical closure and costs of forgiveness (Study 3a)

\begin{tabular}{lcccccc}
\hline \multicolumn{1}{c}{ Scale } & $\chi^{2}$ & $\chi^{2} / \mathrm{df}$ & RMSEA & SRMR & GFI & CFI \\
\hline 1. Historical closure & 89.93 & 2.99 & .04 & .02 & .98 & .94 \\
2. Costs of Forgiveness & 24.40 & 1.02 & .004 & .02 & .99 & 1.00 \\
\hline
\end{tabular}

\section{Between society differences on intergroup forgiveness}

Analysis of variance (ANOVA) was used to assess the impact of society on intergroup forgiveness, testing Hypothesis 1 . There was a significant difference in the intergroup forgiveness score between the six societies (main effect for society: $\left.F(5,1191)=44.81, p<.001, \eta^{2}=.16\right)$. As expected the Chinese participants were least willing to forgive the atrocities committed by Japan during WW II $(M=3.99, S D=.89)$, and the French participants were the most willing to forgive Germans for their wartime atrocities $(M=5.45, S D=1.12)$. Furthermore, the Chinese participants were significantly lower on forgiveness than the Taiwanese $(M=4.62, S D=.86)$ and Filipino participants $(M=4.72, S D=1.04)$. Additionally, the French participants were significantly more forgiving than the Russian $(M=4.75, S D=1.27)$ and Polish $(M=5.10, S D=1.04)$ participants. Whereas the Taiwanese and Filipino participants did not differ on forgiveness, the Russian and Polish participants did differ significantly. These findings confirm the first context effects hypothesis $\left(\mathrm{H}_{1}\right)$ that intergroup forgiveness for historical atrocities is significantly different between the six societies.

\section{Summary of between society differences on other variables}

See Table 5.2 for significant differences on other variables. Chinese participants had less closure with the past, assigned more guilt, were angrier, and had less trust towards Japanese than Taiwanese or Filipino participants. However, 
Chinese participants perceived less costs of granting forgiveness than Taiwanese or Filipino participants, which is a surprising finding.

French participants perceived less costs of granting forgiveness, assigned less guilt, were less angry, and had more trust towards Germans than Russian or Polish participants had. There was no difference between French and Russian participants on historical closure, but Polish participants were different from Russian participants. Russian participants reported slightly higher historical closure than French participants. Chinese participants reported stronger identification with their national group than Taiwanese or Filipino participants. French participants identified less strongly with their national group than Russian and Polish participants. Therefore, there were significant context effects on these variables as well. In conclusion, the findings show a significant amount of variance between the societies.

\section{Relationships among concepts}

I tested the relationships between the new variables "historical closure" and "costs of forgiveness" with the dependent variable (intergroup forgiveness) and established psychological variables (see Table 5.4 for correlation matrix). I calculated Pearson-Product-Moment correlations. Hypothesis 2 proposed positive links between historical closure, trust and intergroup forgiveness, with the costs of granting forgiveness, collective guilt assignment, group-based anger and national identity. As expected historical closure was significantly positively associated with intergroup forgiveness and outgroup trust. Costs of granting of forgiveness were significantly positively related to collective guilt assignment, group-based anger and national identity. Overall, participants who perceived the past as being a historical event that does not have an impact on the future (i.e. they had high 
historical closure), were also higher on intergroup forgiveness and higher on trust.

Participants who perceived low costs of forgiveness assigned less guilt, were less angry and had a lower identification with their national group.

Moreover, hypothesis 3 tested negative links between historical closure and costs of granting forgiveness, collective guilt assignment, group-based anger and national identity. Costs of granting forgiveness were proposed to be negatively related to outgroup trust and intergroup forgiveness. Overall, historical closure was significantly negatively associated with the costs of granting forgiveness, collective guilt assignment, group-based anger and national identity. Furthermore, costs of granting forgiveness were significantly negatively related to intergroup forgiveness and outgroup trust. These results confirm $\mathrm{H}_{2}$ and $\mathrm{H}_{3}$ and provide empirical evidence that the new concepts assessed have convergent validity.

Table 5.4

Intercorrelations between Scales for overall Sample $(N=1197, \text { Study } 3 a)^{9}$

\begin{tabular}{lcccccc}
\hline \multicolumn{1}{c}{ Scale } & 1 & 2 & 3 & 4 & 5 & 6 \\
\hline 1. Intergroup forgiveness & - & & & & & \\
2. Historical closure & $.40^{* * *}$ & - & & & & \\
3. Costs of Forgiveness & $-.43^{* * *}$ & $-.10^{* * *}$ & - & & & \\
4. Guilt & $-.50^{* * *}$ & $-.41^{* * *}$ & $.23^{* * *}$ & - & & \\
5. Anger & $-.52^{* * *}$ & $-.31^{* * *}$ & $.33^{* * *}$ & $.52^{* * *}$ & - & \\
6. Trust & $.43^{* * *}$ & $.25^{* * *}$ & $-.25^{* * *}$ & $-.36^{* * *}$ & $-.35^{* * *}$ & - \\
7. Identity & $-.23^{* * *}$ & $-.17^{* * *}$ & $.14^{* * *}$ & $.34^{* * *}$ & $.35^{* * *}$ & $.15^{* * * *}$ \\
\hline Note. $*^{* * *} p<.001$, two-tailed & & & & & & \\
\hline
\end{tabular}

\footnotetext{
${ }^{9}$ Due to a possible content overlap of some historical closure and forgiveness items, I tested whether the measurements historical closure and intergroup forgiveness are distinct measurements. I conducted a PCA with oblique rotation. I received three factors explaining $55.18 \%$ of the variance. The forgiveness items loaded on two factors (representing the subscales "forgiving" and "non-forgiving" functioning as one overall forgiveness scale) and the historical closure items loaded on the other factor. Furthermore, when I forced a two-factor solution, all forgiveness items loaded on one factor and the historical closure items loaded on the other. Therefore, intergroup forgiveness and historical closure as measured in this study are empirically distinct constructs.
} 
The above analysis showed that the concepts were meaningfully related. However, it is difficult to get a more holistic perspective based on correlations alone. Therefore, a multidimensional scaling (MDS) procedure seemed appropriate to explore these relationships at a more holistic level. I subjected the scales ${ }^{10}$ from the overall data set to an interval-level MDS procedure between variables with proximity transformations using Proxscal in SPSS 14. I calculated Euclidean distances from z-transformed mean scores of the seven concepts considering 1 up to 4-dimensional solutions. A representation in one dimension accounted for .86 of the dispersion (normalized raw stress .13, Tucker's coefficient of congruence $=.93$ ), for two dimensions.99 of the dispersion (normalized raw stress .01, Tucker's coefficient of congruence $=.99$ ), for three dimensions .99 of the dispersion (normalized raw stress .002, Tucker's coefficient of congruence $=.99$ ), and for four .99 of the dispersion (normalized raw stress .003 , Tucker's coefficient of congruence $=.99$ ). The scree-plot of the normalized raw stress suggested a 2-dimensional solution. Therefore, a two-dimensional representation of the concepts was deemed as most appropriate and best interpretable.

According to Borg and Groenen (1997), MDS configurations can be interpreted in three ways. A MDS space can be partitioned by parallel and straight lines (axial way), by concentric circles (modular way) or by rays originating from a common starting point (polar way). As can be seen in Figure 5.1 the modular way of partitioning the two-dimensional space seemed the most interpretable. The

\footnotetext{
${ }^{10}$ Before employing the MDS, I reversed the mean scores of the scales with a negative correlation with intergroup forgiveness (identity, guilt, anger and costs) so that the multidimensional space would not be distorted by the distinction between negative versus positive dimensions.
} 
construct Forgiveness is positioned in the middle of this configuration, highlighting its central relationship to the other constructs.

The emotional constructs guilt assignment and anger surround forgiveness in Figure 5.1. This is in line with previous research that argues that group-based emotions such as anger are related and proximal to forgiveness. The next circle includes Trust and Historical Closure. It has been labelled as "bridging elements" because it incorporates intermediate elements that bridge the inner emotion (or affect) construct circle with the cognitive constructs that sit in the 'cognitive' outer circle (e.g. perceived costs of forgiveness and identification with one's national group). The exploration of the MDS space is insightful as it shows graphically how the constructs may function holistically in relation to one another.

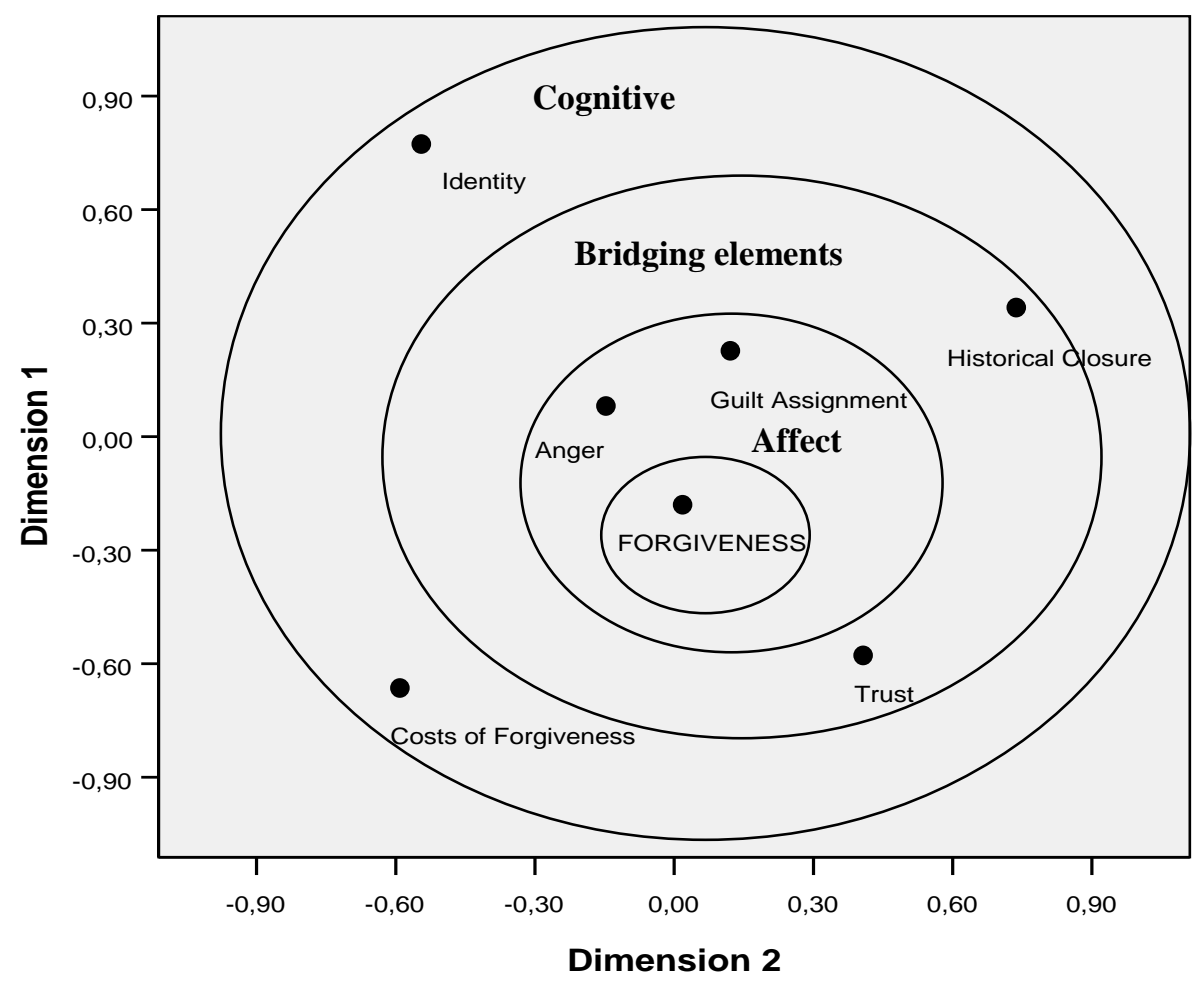

Figure 5.1 Two-dimensional MDS representation overall (Study 3a) 


\section{Similarity of correlation coefficients}

In the following analysis, a $\chi^{2}$ test of r-to-z transformed correlation coefficients examined the similarity of correlation coefficients across societies. There were no differences across the six societies for the correlations between historical closure and intergroup forgiveness $\left(\chi^{2}=6.61, p=.25\right)$, between groupbased anger and historical closure $\left(\chi^{2}=7.90, p=.16\right)$, and between outgroup trust and costs of granting forgiveness $\left(\chi^{2}=6.78, p=.24\right)$.

There were significant differences across the six societies for the following associations: costs of granting forgiveness and intergroup forgiveness $\left(\chi^{2}=18.18\right.$, $p<.01)$, collective guilt assignment and historical closure $\left(\chi^{2}=12.99, p<.05\right)$, collective guilt assignment and costs of forgiveness $\left(\chi^{2}=17.08, p<.01\right)$, groupbased anger and costs of forgiveness $\left(\chi^{2}=28.29, p<.001\right)$, and outgroup trust and historical closure $\left(\chi^{2}=12.97, p<.05\right)$. This means that the strength of these associations varied significantly across the samples. The direction of the associations, however, was the same. The differences are as follows.

The associations between the new concept historical closure and intergroup forgiveness and group-based anger were stable across the six societies; whereas the relationships varied between historical closure and collective guilt assignment, and outgroup trust. Furthermore, the association between costs of granting forgiveness and outgroup trust was similar across the societies. In contrast, the relationships between costs of granting forgiveness and collective guilt assignment (not existent in Taiwan and China), anger and intergroup forgiveness (very strong in France $r=-.57, p<.001$ ) differed between the societies. 
While the overall correlational analyses confirmed predictions about the general associations between these variables, the MDS space showed an interpretable depiction of their relationships. The $\chi^{2}$ test showed that some of these associations differ significantly in their strength across the societies. The question is how the differences across societies can be explained. To resolve this question, I aim to employ a cross-level analysis as outlined in Study $3 b$ to investigate the impact of contextual variables on the patterns revealed.

\section{Identity}

Overall, identification with one's national group partially mediated the relationship between historical closure and forgiveness (Sobel z-value $=4.42, p<$ .001 ) ; and between costs of granting forgiveness and forgiveness (Sobel z-value $=$ $-3.94, p<.001)$. I also tested the mediation within each society and found that Poland drives the overall mediation for identity between historical closure (Sobel z-value $=-1.90, p=.06)$, costs of forgiveness (Sobel z-value $=1.99, p<.05)$ and intergroup forgiveness. In all other societies, identity was not a significant mediator. In the context of post WW II relations in this study, national identity does not contribute significantly to a better understanding of intergroup forgiveness process. $\mathrm{H}_{4}$ was only partially confirmed.

\section{Summary and Conclusion}

A remarkable amount of between society differences were found on intergroup forgiveness and almost all other concepts assessed in this study. The differences found can be explained by the societal context. The low intergroup forgiveness score for the Chinese participants and the high intergroup forgiveness 
score for the French participants indicate that the social representation of the past influences how forgiveness varies.

The new variables historical closure and perceived costs of granting forgiveness have meaningful associations with established psychological variables and provide convergent validity for these concepts overall. However, the strength of association differs between societies. The MDS shows that historical closure in particular can have a bridging function between emotions very close to intergroup forgiveness, and cognitions that are more distal from intergroup forgiveness.

When tested as a mediator, identity did not significantly explain variance in intergroup forgiveness. Several reasons may account for this finding. Identity as assessed in this study might have measured a qualitatively different construct in each society and therefore was associated with different outcomes of the identification process. The aim of the next section is to explain why there were differences across the six societies by employing (a) multiple hierarchical regressions and (b) cross-level operator analysis (CLOP).

\section{Study 3b: Intergroup forgiveness and the historical context: Multiple hierarchical regressions and cross-level operator analysis (CLOP)}

The central aims of this study were two-fold. First, I investigated both contexts separately with hierarchical multiple regressions in order to examine differences and similarities across these two contexts. The new variables "historical closure" and "costs of granting forgiveness" were tested for their contribution to explain variance on intergroup forgiveness above and beyond established predictors across the two contexts.

The second aim was to explore the contribution of societal context variables related to the historical background to explain variations at the 
individual level on intergroup forgiveness, using a cross-level operator analysis (CLOP; James \& Williams, 2000). Ideally, hierarchical linear modeling (HLM) would be best to address this research objective, as this is a multi-level problem. HLM is a relatively new statistical technique that partitions the variance into within (individual level data - level 1) and between units (context/society level data - level 2) to explain variations at level 1. However, HLM has some requirements that need to be met in order to perform it: HLM requires at least 30 individuals (level 1) nested within at least 20 (if possible 30) units at level 2 (for example the need of individual-level data from 20 societies to gain 20 data points at level 2) (see Bassiri, 1988). Hence, HLM is not appropriate for this data.

Fischer (2008) employed an alternative and older statistical technique that is the "cross-level operator analysis" which is a version of regression analysis (CLOP; James \& Williams, 2000). They argued that CLOP is conceptually the same as hierarchical lineal modeling (HLM) and can be conducted if the number of level 2 units are too limited and therefore prevent the application of HLM (Raudenbush \& Bryk, 2002). The only difference between a usual regression procedure and CLOP is that each member of a given society is assigned the same contextual-level score (equivalent to level 2 in HLM) for the concepts of interest (e.g. number of apologies). However, there are some disadvantages using this approach and the results need to be treated with caution. In CLOP the confidence interval is very small due to the low degrees of freedom, which restricts the number of predictors that can be investigated at the same time. The small confidence interval can lead to an increase in Type-I error. CLOP is, nevertheless, a useful tool to explore the meaningfulness of context-level predictors, when HLM cannot be used. 
Therefore, a CLOP was applied to take societal context into account and to explore how it influences the intergroup forgiveness process. After establishing propositions about the relationships between the concepts of interest and identifying differences between the 6 societies (ANOVA revealed 16\% of between society differences on intergroup forgiveness in Study 3a), the next step was to find explanations for these differences. Previous research has been conducted at one level, primarily the individual level. However, contextual variables may have a crucial influence on the differences at the individual level.

The 'context' of interest in this study was the historical background of the society within which participants were nested. Context reflecting the historical background of a society can be represented by objective variables like the number of official apologies issued from 1990, the salience of WW II in collective remembering (see Páez et al., 2008) and evaluation ratings of WW II.

In the current study, the number of issued apologies during the last 20 years was used as an objective society-level indicator for the level of unresolved issues due to WW II. That is, the more apologies that were needed to address past harm done during the last two decades, the less likely forgiveness will be reported. Arguably, resolutions and compensations for harm done during WW II were more successful in the European context than in the East Asian context (Hein \& Selden, 2000; Oliner, 2008). Therefore, it could be speculated that the more official apologies were issued, the less likely it seems that disputes over the past harm done have been resolved and the less likely forgiveness will be reported. Additionally, it can be assumed that the less WW II is recalled in a society, the more likely its citizens will be willing to forgive, since there probably was a departure from the past. Finally, the evaluation ratings of WW II may affect 
the level of forgiveness. It could be assumed that perceptions that WW II is negative will be associated with more willingness to forgive. These assumptions are treated as guiding research questions.

\section{Method}

\section{Participants}

The same sample was the same as reported in Study 3a

\section{Instruments}

The same instruments are used for these analyses as reported in Study $3 \mathrm{a}$.

\section{Society-Level Contextual Variables ${ }^{11}$}

Number of apologies. The number of apologies from 1990 until 2003 was used for the analysis. Dodds (2003) compiled a comprehensive chronological list of political apologies. Only apologies were counted that were addressed to societies in this study. In the last 20 years, Japan issued 29 apologies addressed to either China or all East Asian nations that suffered during Japan's war of aggression. During the same period, Germany issued one apology addressed to Poland. The absolute numbers of apologies were used.

Recall of WW II. The salience of WW II was determined by using the percentage participants from each sampled country who free recalled WW II when asked to list the most important events in world history, taken from a study conducted based on sample of 3,322 university students across 22 societies (Páez et al, 2008). The percentages were standardised to z-scores.

\footnotetext{
${ }^{11}$ A preliminary analysis at the country level revealed significant correlations between the contextual variables and intergroup forgiveness. Intergroup forgiveness was negatively linked to the number of apologies $(r=-.29, p<.001)$, negatively associated with evaluation ratings of WW II $(r=-.14, p<.001)$ and the recall of WW II $(r=-.09, p<.01)$.
} 
Evaluation ratings of WW II. The evaluation ratings of WW II were taken from the study conducted by Páez et al. (2008).

A cross-level operator analysis was conducted with these society-level context variables in order to evaluate the contribution of these cross-level operators in explaining between society variance.

\section{Analytical Strategy}

Multiple hierarchical regressions

First, multiple hierarchical regressions were used in order to compare the East Asian and European context. Dummy codes were created for each society to analyze society-level effects. Two dummy codes were created (1 for the specific society, 0 for the other societies) for calculations with the three East Asian and three European societies (Cohen, Cohen, West, \& Aiken, 2003).

I controlled for gender and age effects and entered the demographic information first into the regression (Step 1). The second block were the dummy codes for the societies, the third block were the established predictors collective guilt assignment, anger, national identity, and outgroup trust (Step 2). The variables costs of granting forgiveness and historical closure were entered last in order to evaluate their unique contribution (Step 3). The variance inflation factor (VIF) was below 3, indicating no problems with multicollinearity.

\section{Cross-level operator analysis (CLOP)}

Second, one objective was to explore the contribution of societal context variables to explain differences on intergroup forgiveness using CLOP. In this analysis level 1 units are the individuals and the level 2 units are the six societies. 
The variables representing level 2 are societal context variables (such as number of apologies).

The impact of the society-level context variables was tested using CLOP. In this type of hierarchical regression individual demographics (gender: $1=$ female, age) were entered first (Step 1, level 1) as control variables. At Step 2 (level 1) established intergroup-level predictors (collective guilt assignment, anger, trust) were entered, and the new variables (historical closure, and costs of forgiveness). In Step 3 (level 2) predictors representing the historical context were entered.

\section{Results}

\section{Multiple Hierarchical Regressions}

Multiple hierarchical regressions tested the contribution of the new variables to explain variance in intergroup forgiveness separately for the East Asian (see Table 5.5) and European context (see Table 5.6). The separate regressions were conducted in order to investigate differences between the two contexts.

\section{East Asian context}

Please see Table 5.5 for detailed overview of the regression. The first model revealed neither gender nor age effects in the East Asian context. Gender effects appeared in the second step when society-level effects were controlled for, but this could be a suppressor effect since the zero-order correlation between gender and forgiveness was non-significant $(r=-.02$, n.s.). The same effect may have driven the age differences that emerged in the third step. Nevertheless, I examined the differences on intergroup forgiveness in gender through simple independent samples t-tests. This analysis only revealed significant differences between male 
and female participants in China $(t(198)=2.88, p<.01)$ and marginally significant differences in the Philippines $(t(198)=1.71, p=.09)$. No age differences were found. Interestingly, in China and the Philippines male participants were more forgiving than female participants, which is an unusual finding. This difference can be potentially explained by the disputes over the comfort women issues in which mostly women from China and the Philippines were affected.

All dummy codes in Step 2 were significant, indicating that there were differences between the Philippines, Taiwan and China on intergroup forgiveness $\left(\Delta R^{2}=.11, p<.001\right)$. The society-level effects remained stable and mostly significant even after all variables were entered, meaning that there were context effects for these societies even after controlling for all psychological predictors. The established predictors were significant contributors to the explanation of variance in intergroup forgiveness in Step 3, except for national identity. In Step 4 when the new concepts were entered the $\beta$-weights for the established predictors decreased, but were still significant. Historical closure was a positive predictor and costs for granting forgiveness a negative predictor of intergroup forgiveness. Historical closure and costs of forgiveness explained additional 9\% of the variance in intergroup forgiveness $\left(\Delta R^{2}=.09\right)$, which is a significant increase in explained variance $\left(F_{\text {change }}(2,610)=45.25, p<.001\right)$. 
Table 5.5

Multiple Hierarchical Regression, East Asian context (N=624, Study 3b)

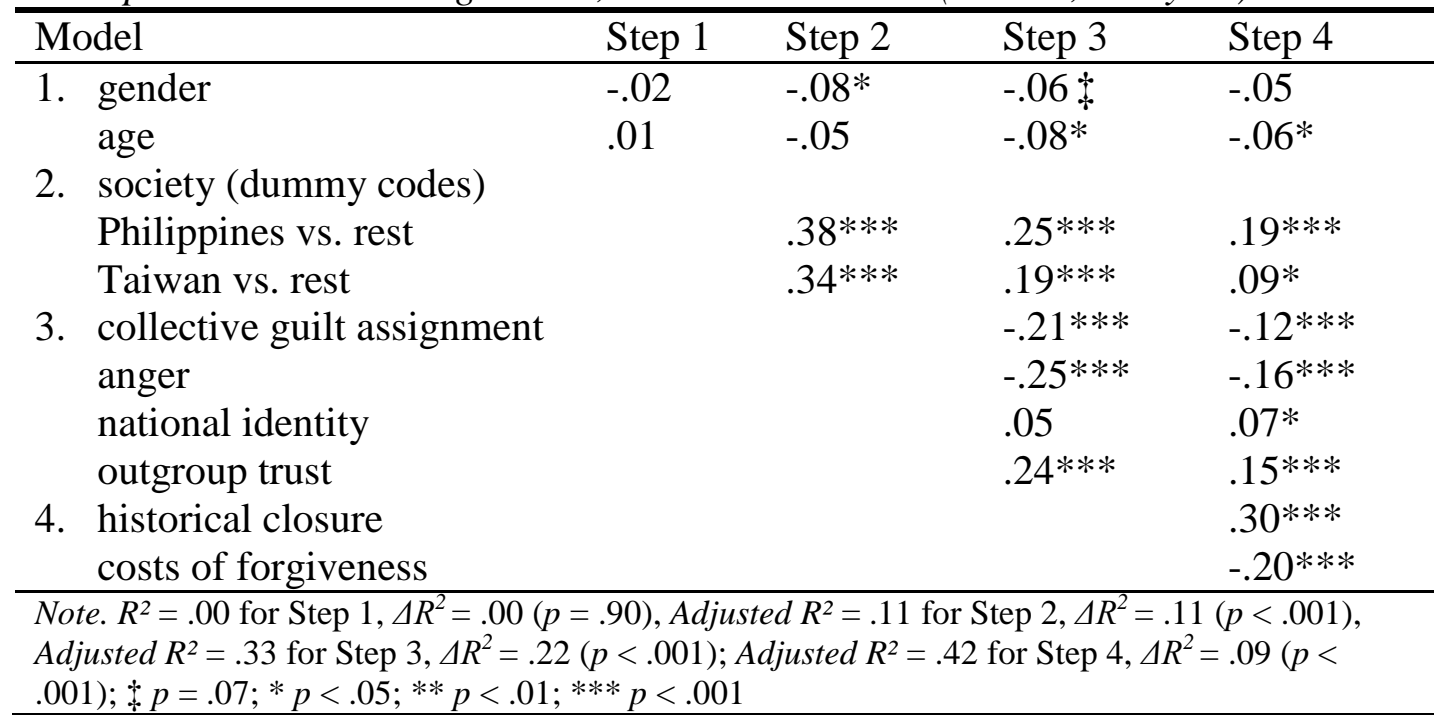

European context

In the European context (see Table 5.6), I found significant gender and age effects in the first step. In contrast to the East Asian context, older participants were more willing to forgive than younger participants, and female participants were more willing to forgive than male participants. These findings are consistent with previous research in this area (e.g. Hewstone et al, 2004). However, these effects disappeared in the second step when society-level effects were controlled for.

All dummy codes in Step 2 were significant, indicating society-level effects on intergroup forgiveness $\left(\Delta R^{2}=.06, p<.001\right)$. The society-level effects disappeared after the last step, indicating that the variability between the societies in the European context could be explained by the psychological variables entered. This contrasts with the findings in the East Asian context. The last step which tested the unique contribution of the new variables of historical closure and the costs for granting forgiveness explained variance in intergroup forgiveness 
was significant $\left(F_{\text {change }}(2,559)=52.11, p<.001\right)$, explaining $10 \%$ of the variance in intergroup forgiveness $\left(\Delta R^{2}=.10\right)$.

Table 5.6

Multiple Hierarchical Regression, European context $(N=573$, Study $3 b)$

\begin{tabular}{|c|c|c|c|c|}
\hline Model & Step 1 & Step 2 & Step 3 & Step 4 \\
\hline \multirow{2}{*}{$\begin{array}{l}\text { 1. } \text { gender } \\
\text { age }\end{array}$} & $.10^{* * *}$ & $.08 t$ & .06 & .05 \\
\hline & $.15^{* * * *}$ & .06 & .06 & .03 \\
\hline \multicolumn{5}{|l|}{ 2. society (dummy codes) } \\
\hline Russia vs. rest & & $-.25 * * *$ & $.10 *$ & .06 \\
\hline Poland vs. rest & & $-.13 * *$ & $.13 * *$ & .07 \\
\hline \multirow{4}{*}{$\begin{array}{l}\text { 3. collective guilt assignment } \\
\text { anger } \\
\text { national identity } \\
\text { outgroup trust }\end{array}$} & & & $-.25 * * *$ & $-.18 * * *$ \\
\hline & & & $-.31 * * *$ & $-.18 * * *$ \\
\hline & & & -.02 & -.001 \\
\hline & & & $.24 * * *$ & $.20 * * *$ \\
\hline \multirow{2}{*}{$\begin{array}{l}\text { 4. historical closure } \\
\text { costs of forgiveness }\end{array}$} & & & & $.13 * * *$ \\
\hline & & & & $-.33 * * *$ \\
\hline \multicolumn{5}{|c|}{$\begin{array}{l}\text { Note. } R^{2}=.02 \text { for Step } 1, \Delta R^{2}=.02(p<.001), \text { Adjusted } R^{2}=.06 \text { for Step } 2, \Delta R^{2}=.04(p<.001), \\
\text { Adjusted } R^{2}=.37 \text { for Step } 3, \Delta R^{2}=.31(p<.001) ; \text { Adjusted } R^{2}=.47 \text { for Step } 4, \Delta R^{2}=.10(p<.001) ; \\
\$ p=.06 ; * p<.05 ; * * p<.01 ; * * * p<.001\end{array}$} \\
\hline
\end{tabular}

The next section will discuss the differences between the two contexts and the sampled societies identified in the cross level operator analysis (CLOP).

\section{Cross level operator analysis (CLOP)}

The number of apologies, evaluations of WW II and salience of WW II represented the 'historical context' in this analysis. Overall, the results indicated that the numbers of apologies issued during the last 20 years was the only significant contributor that explained significant variance in intergroup forgiveness (see Table 5.7). Forgiveness was more frequently reported in societies in which there were fewer apologies issued during the last two decades. The salience of WW II and the evaluations of WW II remained non-significant.

The number of apologies as a contextual variable alone explained $0.5 \%$ of the variance in intergroup forgiveness. This seems low; however, this amount of explained variance was significant $\left(F_{\text {change }}(3,1180)=3.46, p<.05\right)$, and still 
noteworthy. A reason that it seems so low is that there was no variance partition within and between units with CLOP, as both levels (individual and unit-level), explained variance at the individual level (Fischer, 2008; Klein, Bliese, Kozlowski, Dansereau, Gavin, Griffin, et al., 2000).

Consequently, CLOP reveals between-units effects that look smaller than they would with HLM, since higher-level variables explain the total variance in the dependent variable and not only between-unit variance, as it would be the case in HLM (Fischer, 2008). It is also important to regard the unique contribution of the contextual variable in view of the variation between the societies that needed explanation (ANOVA in Study 3a revealed $16 \%$ of between society variation). The context-level variable number of apologies did explain significant variance. However, a large amount of this variance can be explained by the intergroup variables $\left(\Delta R^{2}=.45, p<.001\right)$. This partially explains why the European context between-society effects disappeared after the established psychological predictors were entered into the hierarchical regression equation. 
Table 5.7

Summary of hierarchical regression analysis of variables predicting intergroup forgiveness using cross-level operators (CLOP) (Study 3b)

\begin{tabular}{|c|c|c|}
\hline & Variables & $\begin{array}{l}\text { Intergroup } \\
\text { forgiveness }\end{array}$ \\
\hline \multirow{12}{*}{$\begin{array}{l}= \\
0 \\
\overrightarrow{0} \\
\end{array}$} & & $\beta$ \\
\hline & $\begin{array}{l}\text { Step 1: Demographic controls } \\
\text { gender }(1=\text { female }) \\
\text { age }\end{array}$ & $\begin{array}{c}.02 \\
-.02\end{array}$ \\
\hline & $\Delta R^{2}$ & $.02 * * *$ \\
\hline & $\Delta F$ & $14.52 * * *$ \\
\hline & Step 2: social-psychological variables & \\
\hline & collective guilt assignment & $-.16^{* * * *}$ \\
\hline & anger & $-.19 * * *$ \\
\hline & outgroup trust & $.16^{* * *}$ \\
\hline & historical closure & $.20 * * *$ \\
\hline & costs of forgiveness & $-.26 * * *$ \\
\hline & $\Delta R^{2}$ & $.45 * * *$ \\
\hline & $\Delta F$ & $198.29 * * *$ \\
\hline \multirow{6}{*}{$\frac{d}{0}$} & Step 3: Historical Context & \\
\hline & apologies & $-.10 * *$ \\
\hline & WW II Evaluation & .01 \\
\hline & WW II Recall & -.03 \\
\hline & $\Delta R^{2}$ & $.005^{* *}$ \\
\hline & $\Delta F$ & $3.46 * *$ \\
\hline
\end{tabular}

\section{Discussion}

The aim of the study was to investigate intergroup forgiveness and its psychological antecedents in the social contexts of post-WW II Europe and East Asia. The results revealed significant variations between the six societies on intergroup forgiveness. French participants were significantly more willing to forgive Germans for their war crimes during WW II than Chinese participants were willing to forgive Japanese for their war crimes. Russian and Polish participants differed significantly in their willingness to forgive with Russian 
participants being less willing to forgive than Polish participants. The Filipino and Taiwanese participants did not differ, but were more willing to forgive than Chinese participants were. Russian, Polish, Filipino, and Taiwanese participants were in between France and China. Similar results apply for the differences found on collective guilt assignment, anger, outgroup trust and historical closure. These findings underlined Germany's success in making reparations and restoring relations after WW II, thus making it the more resolved context in terms of dealing with the past compared to East Asia. In East Asia and especially in China there are still historical tensions due to perceived lack of acknowledgment of past misdeeds on the part of Japan (Liu \& Atsumi, 2008), making Chinese participants significantly less forgiving.

This study contributes to the literature on intergroup forgiveness by providing empirical evidence from six formerly victimized nations that the forgiveness process is affected by shared representations of past (Hilton \& Liu, 2008; Liu \& Hilton, 2005). The post WW II context provides an interpretative frame for situations in which individuals from formerly conflicting nations interact, exchange and relate to one another. The social context in which these interactions happen shape the content of how WW II is remembered and interpreted (Liu \& Hilton, 2005; Páez \& Liu, in press). This 'content' is reflected in the measures of historical closure (attitudes towards the past), which triggered collective memory of victimization in WW II by Germany and Japan, respectively, and historical symbols of victimization (Nanjing Massacre in China, Comfort Women in the Philippines, the Holocaust in Europe).

Thus, this study showed that people held different opinions and attitudes towards another group when (a) an objective situation is set into the post WW II 
context, which has implicit meanings; (b) this context is subject to interpretation and is associated with specific psychological content tailored to the situation. Consequently, Chinese were less forgiving than French. The interpretation of the past and its social representation is triggered by the context. This can also be consciously induced by political discourse to create ideologies based on the social representation of the past that serve to mobilize people (see Sibley et al., 2008), or are unconsciously included in decisions about whether or not to trust people from the perpetrator group (Takahashi et al., 2008; Liu et al., under review).

The MDS gave insight into how intergroup forgiveness may function with other constructs. Consistent with the literature, emotional laden constructs such as guilt and anger were positioned close to intergroup forgiveness followed by historical closure and trust. (see Tam et al., 2007). National identity and the perceived costs of granting forgiveness were most distant from intergroup forgiveness. Intergroup forgiveness has specific psychological antecedents. However, the empirical data showed that the social context of the historical tensions exacerbated by more unresolved issues in the Chinese context crucially affected the forgiveness process.

In sum, the importance of context was illustrated by the significant differences in intergroup forgiveness, collective guilt assignment, outgroup trust, anger and historical closure between the six societies. Additionally, the CLOP analysis showed that the number of apologies during the last two decades was a negative predictor for intergroup forgiveness. Staub and Bar-Tal (2003) noted that shared understanding of the past helps to open a dialogue, which is part of the definition of contextualized intergroup forgiveness. The requirement for repeated apologies about unresolved issues due to WW II in the East Asian context may 
indicate that there is minimal shared understanding between the affected nations especially when considering the low historical closure scores generated in this context compared to the European context. Future research could investigate whether this is the case, and the role that shared understanding plays in historical closure and intergroup forgiveness in this context and in Europe.

These findings indicate that context is more than just a description. It can contribute to the understanding of what can influence the process of forgiveness in the aftermath of historical atrocities, such as in WW II. Two new concepts were introduced: historical closure and perceived costs of granting forgiveness. Study 3a demonstrated the predictive power of both new variables. Furthermore, through examining the relationships between established concepts and intergroup forgiveness, there was an indication of convergent validity for these new concepts. Moreover, it can be argued that historical closure is not a political orientation, because it was unrelated to political orientation ${ }^{12}$ in both contexts $\left(r_{\text {Asian }}=.03\right.$, n.s. $; r_{\text {European }}=-.003$, n.s. $)$. However, historical closure was related to national identity in general. Participants who were identifying more with their national group, showed less closure with past. Nevertheless, this association was not robust and did not hold in each society since it was unrelated in Taiwan and France. Thus, it is possible that historical closure is different from historical negation (Sibely et al., 2007) as it embodies attitudes about the past being confined to the past or having a role in the present without giving implications for actions (e.g. a duty to compensate) or guilt, which is the case with historical negation. The meditation analysis also revealed that identity did not seem to affect

\footnotetext{
${ }^{12}$ Political orientation was assessed, but not reported for this study.
} 
the forgiveness process in this study, as SIT/SCT would have predicted, weakening the overall impact of identity.

The investigation of the effects of perceived costs of forgiveness yielded intriguing findings to contribute to the understanding of intergroup forgiveness. This is the first empirical study that took the costs of forgiveness into account quantitatively. Most previous research focused predominantly on benefits and the costs of not being forgiving. The perception of the costs of forgiveness is important in the forgiveness process. It can be an obstacle to engage in a forgiveness process because of fear of the risk of injustices being continued, or the strategic misuse of political statements by the perpetrator (Rata et al., 2009). The formulations of the scale to assess perceived costs of granting forgiveness are quite general in their formulations. However, it is fair to argue that the social context drives the interpretation of the content provided. Consequently, low perceived costs of forgiveness in the East Asian context resulted in lower forgiveness than in the European context. It seems not to matter that the perceptions of costs are low, because this perception did not alter the lack of historical closure. The perceived costs may be low, but the conflict is still there.

It seems plausible that there are shared understandings and widely accepted interpretation of WW II in the European context. Consequently, especially French participants showed comparatively high intergroup forgiveness, low guilt assignment, low anger, relatively high outgroup trust and relatively high historical closure and less perceived costs of forgiveness. Franco-German relationships were among the first after WW II to become friendly or at least less unfriendly again which is evident in textbook commissions starting in 1951 involving French and German historians to gain a shared understanding of the past with the aim to 
reduce negative stereotypes of the former enemy (Ruchniewiecz, 2005; Viedt, 1993).

In contrast, it seems evident that there are different understandings and interpretations of the atrocities during WW II in East Asia present. Chinese participants showed relatively low historical closure, high guilt assignment, high anger, low outgroup trust, and low intergroup forgiveness, which can be interpreted that there are group boundaries between China and Japan created due to a lack of shared or conflicting representation of the past (Liu \& Atsumi, 2008; Brewer, 2001; Raudsepp, 2005). The question remains: how can this dilemma be resolved?

A limiting factor is the cross-sectional nature of the study, which does not allow drawing causal conclusions. However, the findings are very strong and the explained variance by the predictors is reasonably high (adjusted $R^{2}=.49$ ). Furthermore, the study was short of another independent measure of resolved versus unresolved conflict settings. This would be certainly helpful to provide a separate set of indicators that could independently represent the context besides the inferences about historical representations.

History is an important symbolic resource and defines the sources of a conflict, and the lessons learnt from the past (Liu \& Hilton, 2005). The investigation also showed that understanding forgiveness is dependent on various antecedents and contextual variables. The historical context and its interpretation is a part of this complex relationship. The consideration of the social context can help to better understand forgiveness between societies. Future research in this area is important as it can shed light on historically rooted tensions between societies and help to further our understanding about how historical narratives can 
be challenged in order to promote a shared understanding about history and to promote intergroup forgiveness.

Study 4 will focus on the perpetrator perspective, since an intergroup conflict or a conflict in general rooted in the past is only relevant when the perpetrating part is existent. The whole intergroup forgiveness process includes the perpetrator, as it is argued that forgiveness at this level is a bilateral process rather than only residing in one party. 


\section{Chapter 6}

\section{Study 4: Perpetrator perspectives - German and Japanese willingness to make amends}

\section{Introduction and Outline of Study 4}

This study investigates perpetrator perspectives in the context of post WW II. Studies looking at the perspectives of perpetrators who do not inhabit the same region as the former victims are rare. The majority of research has investigated within-nation situations where one group has at some stage been the victim and at another stage been the perpetrator (most prominent example: Northern Ireland, see also Chapter 2), or where there was a clear past victimized party in an on-going conflict (e.g. the right-wing regime of Pinochet versus the left-wing conflicts in Chile).

It is equally important to examine a conflict where there is a clear perpetrator from the perpetrators' perspective to fully comprehend the forgiveness process. As previously elaborated, WW II is a key event in human history (Liu et al., 2005). WW II is also long enough in the past so that it ensures a certain temporal distance between the atrocities committed during this gruesome war and the present. Therefore, it allows for investigating whether time indeed heals all wounds and whether we have learnt a lesson from the past.

I have already given a historical overview of how Germany and Japan have dealt with their past in Chapter 1. Germany has dealt with her past differently compared to Japan and official outlets or governmental bodies communicated this knowledge accordingly (Hein \& Selden, 2000; Liu \& Atsumi, 2008; Oliner, 2008). The differences in the public handling of the past are mostly due to the different way that WW II ended for Germany and for Japan, and 
different interpretations of the consequences of WW II. Whereas in Germany WW II ended in a ground-zero, in which everything had to be rebuilt under the guidance of the allies. Japan "never experienced any clear break with the pre-war regime" and leading politicians and the Emperor remained in high political positions and in power long after WW II ended (Thelle, 2007, p. 83).

This research is embedded within the post World War II context. As already demonstrated in the victim perspectives discussed in Chapter 5, the historical context helps in explaining the way in which specific constructs are expressed (high guilt assignment and low forgiveness in China, low guilt assignment and high forgiveness in France). For the perpetrator perspective similar effects are anticipated with respect to the impact of historical context. Therefore, I expect significant differences in the outcome variable willingness to make amends. Furthermore, I expect differences in collective guilt and shame feelings between Germany and Japan. It is arguable that German participants will express less guilt and shame feelings than Japanese participants will, since the resolution of the historical past has progressed further in Germany than in Japan (Hein \& Selden, 2000). Although it is often reported that Japan is in denial or at least reluctant to admit any wrong-doing during WW II (Hein \& Selden, 2000; Oliner, 2008). I would like to point out that this is usually what is publicly reported, and may not convey private sentiment.

As outlined in Chapter 2, Japan is faced with polemical representations of her past deeds vis-à-vis her neighbours. What is known outside Japan or what people perceive constitutes Japan, does not necessarily represent Japan as a whole (Ogawa, 2000). There is substantial international debate about some of the textbooks endorsed by the Japanese Ministry of Education, which downplays 
Japan's atrocities during WW II. However, what is usually not reported is that there are opinions within Japan arguing against these historical representations in form of textbooks (e.g. mostly teachers or grassroots movements, Ogawa, 2000). In the era of fast exchange of information via the internet, it is highly likely that Japanese people have come across different opinions on the role of Japan as an aggressor during WW II. Nozaki (2003) argued that it is evident that an ongoing cultural and political struggle due to the textbook controversies and Japan's dealing with the past in general is present within Japan. This struggle within Japan can have consequences for Japanese opinions about compensation, closure with past and guilt feelings today.

As generally reviewed in Chapter 2, collective guilt feelings arise when someone senses that what the ingroup did in the past was wrong. These guilt feelings especially arise when there is a feeling of controllability over the past events. In other words, people will feel guiltier when they think harm done could have been prevented and was under the control of the ingroup. On the other hand, collective shame focuses on factors that are not controllable, like the characteristics of the group being exposed as weak or even disgusting (Branscombe \& Doosje, 2004; Brown et al., 2008). Furthermore, according to Páez, Marques, Valencia, and Vincze (2006) collective guilt and shame reflect the emotional side of collective responsibility and are related to actual or symbolic reparation efforts.

Representative samples of public opinions in Germany as reported in Páez and colleagues (2006) showed that only 32\% of Germans surveyed in 1951 thought that Germany was guilty for the war compared to $62 \%$ in 1967 . Although contradictory, people directly involved in collective harm done as perpetrators or 
passive bystanders, do not feel either guilty or ashamed (Páez et al., 2006). Therefore, since collective guilt and shame feelings are related to collective rather than personal actions, it is more likely that new generations which were not involved in or not connected to historical crimes experiences these group-based emotions (Rensman, 2004, cited in Páez et al., 2006).

Brown and colleagues (2008) established links between collective guilt and shame feeling and the willingness to repair damage in their longitudinal study. They demonstrated that collective guilt promotes the willingness to make amends longitudinally whereas collective shame only predicted willingness to repair cross-sectionally. Thus, since I operate with cross-sectional data collective guilt and shame should predict willingness to make amends. Furthermore, there should be differences in the willingness to make amends between Japanese and German context. Doosje, Branscombe, Spears, and Manstead (1998) argued that it is more likely to show amending cognitive responses than actual behaviour, since the past injustices and harm done cannot be changed. Hence, I expect the expression of cognitive component of the willingness to make amends to be higher than the behavioural one.

The anthropological literature suggests that Japan is a so-called "shame" culture and Germany a "guilt" culture, but this is also contested (Creighton, 1990). This distinction, which dates back to Benedict (1946), has been particularly applied when distinguishing between the way in which Japan and Germany have dealt with the past (Páez et al., 2006). Conrad (2003) argued that the conventional picture of Japan as being innately unable to critically handle being the aggressive party of historical past is a part of Japan's national character. Therefore, one could expect that Japanese participants experience more shame rather than feelings of 
guilt, compared to a "guilt" culture, which Germany is claimed to be. However, there is some research showing that shame and guilt feelings are equally exposed when social norms are violated in collectivistic and individualistic cultural contexts (e.g. Bierbrauer, 1992). Nevertheless, this kind of research is scarce, and has been conducted at the interpersonal level without considering implications for intergroup research.

SIT (Tajfel \& Turner, 1979) and SCT (Turner et al., 1987) have been applied to make predictions summarized in the reviewed research on intergroup forgiveness in Chapter 2. Conclusive results found that the level of identity was the driving force behind feeling guilty for past harm done. Based on SIT/SCT, it is expected that people who identify more strongly with their nation, and are confronted with the challenge of maintaining a positive social identity, would do so by distancing themselves from the harm done in the past and expressing less collective guilt feelings than people who report low national identification. The reverse effect is expected when people identify with a more inclusive identity (here being globally conscious) in line with SCT (Turner, 1999).

Following up the concept of historical closure that I introduced in Chapter 5, I will test the relationships with established psychological variables (such as collective guilt and shame), new concepts (such as historical closure) and the willingness to make amends. Historical closure conceptualized as an attitude towards the past is expected to be generally low, since the conflict plays a substantial part in controversial political debates in both contexts.

In summary, I will investigate the following guiding research hypotheses. There should be mean differences between Germany and Japan on all assessed variables and on the outcome variable willingness to make amends (historical 
context hypothesis, $\mathrm{H}_{1}$ ). Due to existing theorizing about Japan being a "shame" culture and Germany being a "guilt" culture, I would expect Japanese participants to report more shame feelings than German participants $\left(\mathrm{H}_{2}\right)$. Furthermore, I expect higher means for the cognitive component of the willingness to make amends $\left(\mathrm{H}_{3}\right)$ than the behavioural component. Collective guilt and shame should predict the willingness to make amends.

Moreover, participants identifying more with their nation should experience less guilt and shame feelings, and participants embracing a more inclusive identity should feel more guilty and ashamed of the historical past (identity hypothesis, $\mathrm{H}_{4}$ ). Since this is a novel undertaking in comparing these two contexts, the nature of this study is explorative, but guided by following hypotheses:

Hypothesis $1\left(\mathrm{H}_{1}\right.$-historical context): There should be mean differences between Germany and Japan on all assessed variables and on the outcome variable willingness to make amends.

Hypothesis $2\left(\mathrm{H}_{2}\right)$ : Japanese participants should exhibit more shame feelings than German participants.

Hypothesis $3\left(\mathrm{H}_{3}\right)$ : The expression of the cognitive component of the willingness to make amends should be higher than the behavioural one.

Hypothesis $4\left(\mathrm{H}_{4}\right)$ : Participants identifying more with their nation should feel less guilt and shame feelings, and participants embracing a more inclusive identity should feel more guilty and ashamed of historical misdeeds.

In general, I aim to answer following research question:

What do the new concepts of historical closure, benefits of seeking forgiveness, face concerns contribute to the prediction of the willingness to make amends? 


\section{Method}

\section{Participants}

An anonymous questionnaire was administered to 266 university students (mean age $=22.26, S D=3.58$, female $=50 \%, 1.1 \%$ did not disclose their gender $)$ in Japan (Osaka, $\mathrm{N}=132$ ) and Germany (Hamburg, $\mathrm{N}=134$ ) (for an overview of sample characteristics see Table 6.1). All questionnaires were administered in the native language of each participating country. A bilingual committee approach was employed in order to ensure correct translation of the survey (van de Vijver \& Leung, 1997). The proportion of males was relatively high in Germany because the data was collected from a university of the German Federal Armed Forces.

Table 6.1

Sample description for Japan (N=132) and Germany (N=134) (Study 4)

\begin{tabular}{lcccc}
\hline \multirow{2}{*}{ Society } & \multirow{2}{*}{$\mathrm{N}$} & \multicolumn{2}{c}{ Gender } & Age \\
\cline { 3 - 4 } & & Female & Male & M (SD) \\
\hline Japan & 132 & $69.2 \%$ & $30.8 \%$ & $21.09(3.94)$ \\
Germany & 134 & $32.3 \%$ & $67.7 \%$ & $23.39(2.78)$ \\
\hline Total & 266 & $50.6 \%$ & $49.4 \%$ & $22.26(3.58)$ \\
\hline
\end{tabular}

Instruments

Participants completed an anonymous questionnaire assessing self-report measures of interest and demographic information (e.g. age, gender). Please see Appendices C1 (German version) and C2 (Japanese version with English wording) for all items reported in the following section.

Willingness to make amends. This 5-item scale was constructed for this study in order to capture two components of willingness to make amends: a 
behavioural and cognitive component. The five items were subjected to an EFA with principal component analysis (PCA) and oblique rotation in order to investigate the data structure for each country separately. The factor analysis showed a clear two-factor solution that explained $81 \%$ of the variance in Germany and $80 \%$ of the variance in Japan. Behavioural items loaded on the first factor with loading ranging from .77 to .94 in Japan, and from .76 to .90 in Germany and did not load on the second factor (cross-loadings $<.30$ in both countries). The cognitive items loaded on the second factor (loadings .86 and .94 in both countries) and had cross-loadings below. 30. This indicates distinct two factors, which can be interpreted as a behavioural and cognitive component.

The behavioural facet of the willingness to make amendments consisted of 3 items: "I am willing to show my support for former overseas Asian (nonJapanese) victims of Japan's warfare through wearing badges with political statements", "I am willing to be actively involved in an institution that runs information stalls to support the demand for an official acknowledgement of Japan's harmful past actions towards other (non-Japanese) Asians" and "I am willing to recruit people to sign petitions that supports compensation claims addressed to Japan's government". The internal consistencies for this scale were .86 in Japan and .83 in Germany. A higher value denotes greater willingness to make amends.

The cognitive aspect of the willingness to make amendments comprised of 2 items: "It's important to support claims of compensation of former overseas Asian (non-Japanese) victims of Japan's harmful past actions during the occupation of Asia and World War II" and "I think that former overseas Asian (non-Japanese) victims of Japan's harmful past actions deserve some form of 
compensation from Japan”. Cronbach's alpha was .78 for Japan and .83 for Germany respectively. A higher value indicates greater agreement for the support of the claims. These two factors are structurally equivalent (for cognitive amends: Tucker's Phi=.99; for behavioural amends: Tucker's Phi=.97) across Japan and Germany.

Historical closure. This scale is the same as used for the victimized societies. However, two items were deleted, because they were inconsistent across the two countries. The four item-scale had modest reliability $\left(\alpha_{\mathrm{Japan}}=.71\right.$ and $\alpha$ Germany $=.57)$. The historical closure scale assesses the degree to which the participants perceive the past as a part of the present and future, as opposed to being unconnected to the present/future. Sample items are (Japanese context): "Discussions about apologizing and forgiving related to Japan's harmful past actions towards other Asians are not important any more", "Grievances and resentment related to Japan's harmful past actions towards other Asians will be carried to the next generation" (reversed-scored), "Today there is no need to talk so much about the harm committed by Japan in the past," and "Other Asians' stories of surviving Japan's harmful past actions make me feel like the wounds are still open today". A higher value means greater closure with the past.

Face concerns. Face is an important concept in Asian cultures and may influence the way people from different cultures negotiate (see for example TingToomey, 2005). The concept of concern with losing face was measured with four items that were created for this study. The items were "If Japan's government acknowledges the harm done during the occupation of Asia and World War II, Japanese people will lose face", "If Japan's government acknowledges the harm done during the occupation of Asia and World War II, the extent of face loss for 
Japanese people will be irreparable", "It is understandable that Japanese people would be concerned about a loss of face, if official apologies to another country for Japan's war of aggression are issued", and "If the Japanese prime minister seeks forgiveness from other Asian countries, he risks a loss of face for the whole nation". The internal consistency of the scale was adequate, with .65 for Japan and .82 for Germany. A higher value indicates higher face concerns due to the government's handling of the past.

Perceived benefits of seeking forgiveness. This three-item scale was constructed for this study and assessed general benefits of seeking forgiveness. A sample item is "Seeking forgiveness may be the first step towards social harmony between groups.” Internal consistencies were .70 for Japan and .66 for Germany. A higher value denotes the higher the perceptions of benefits.

Outgroup trust. The same instruments were devised as used in Study 3. However, the formulations of the items differed slightly from the items used in Study 3. Outgroup trust was assessed with following items: "Do you think most other Asians would try to be fair?", "Would you say that most of the time other Asians try to be helpful?", and "Generally speaking, would you say that most other Asians can be trusted?" Cronbach's alpha was good ( $\alpha_{\text {Japan }}=.72$ and $\left.\alpha_{\text {Germany }}=.81\right)$. A higher value indicates more trust.

Empathy. Empathy was assessed with these items: "I try to imagine what other Asians have gone through during World War II", "I sometimes think about how other Asians might have felt during World War II", "I am trying to look at things that happened during World War II from the perspective of other Asians", and “Usually, I am able to understand the other Asians' point of view regarding World 
War II". The internal consistencies for this scale were .88 in Japan and .86 in Germany. A higher value denotes higher empathy.

For the outgroup trust and empathy scale an open-ended question was added asking for a specific Asian nation they had in mind while answering. The same applied for the German context only adjusted to suit the German setting.

Collective guilt. Collective guilt was measured with a scale adapted from Branscombe and Doosje (2004). The five items measured the degree to which the participants felt guilty on behalf of Japan or Germany for the war crimes they have committed. A sample item is "I can easily feel guilty for the bad outcomes brought about by Japanese people in the past." Cronbach's alphas were .86 in Japan and .81 in Germany. A higher value denotes greater feelings of guilt.

Collective shame. Four items to measure the degree feeling ashamed for the misdeeds of Japan and Germany respectively were created: "I feel ashamed because Japan committed so many misdeeds against other Asian nations in the past", "I feel ashamed about what our (great) grandparents did during the occupation of Asia, the Sino-Japanese War and World War II", "I feel ashamed when foreigners mention the Nanjing massacre and the Comfort Women Issue", and "Public discussions about past misdeeds make me feel ashamed of my ancestors' past actions". Internal consistencies were good $\left(\alpha_{\text {Japan }}=.86\right.$ and $\left.\alpha_{\text {Germany }}=.85\right)$.

Global Consciousness. This 6-item scale was constructed for this study and assessed the degree of seeing oneself of a part of a global community as opposed to being a part of a specific national group. The items were as follows: "I think of myself as a citizen of the world rather than of one particular country", "Citizens of each country should look after themselves instead of worrying about 
people from other parts of the world" (reverse-scored), "I think of myself as a loyal citizen of my own country rather than as a citizen of the world" (reversescored), "People from each country should mind their own business" (reversescored), "I identify with the human race more than any particular nationality", and "Being a good citizen of the world is an important part of how I see myself". The internal consistencies were moderate for Germany with a Cronbach's alpha of .51, and good for Japan with a Cronbach's alpha of .83. A higher value denotes higher global consciousness.

Identification with one's national group. This is the same instrument used in Study 3. Cronbach's alpha was good $\left(\alpha_{\text {Japan }}=.84\right.$ and $\left.\alpha_{\text {Germany }}=.87\right)$.

\section{Results}

\section{Descriptive statistics and reliabilities}

Table 6.2 shows the descriptive statistics, reliabilities and internal consistencies as well as t-values indicating significant differences on the assessed constructs.

Table 6.2

Means, Standard Deviation, Cronbach's alpha, t-values (Study 4)

\begin{tabular}{lccccc}
\multicolumn{1}{c}{ Society } & \multicolumn{2}{c}{ Germany } & \multicolumn{2}{c}{ Japan } & $\mathrm{t}$ \\
\cline { 1 - 5 } \multicolumn{1}{c}{ Scale } & $\mathrm{M}(\mathrm{SD})$ & $\alpha$ & $\mathrm{M}(\mathrm{SD})$ & $\alpha$ & \\
\hline Amendments (cognitive) & $4.72(1.49)$ & .83 & $4.68(1.37)$ & .78 & -.14 \\
Amendments (behaviour) & $2.14(1.17)$ & .83 & $2.97(1.54)$ & .86 & $4.95^{* * *}$ \\
Historical Closure & $3.43(1.10)$ & .57 & $2.96(1.13)$ & .71 & $-3.49^{* * *}$ \\
Benefits of seeking forgiveness & $5.43(1.11)$ & .66 & $4.82(1.17)$ & .70 & $-4.28^{* * *}$ \\
Guilt & $3.20(1.19)$ & .81 & $4.28(1.19)$ & .86 & $7.39^{* * *}$ \\
Shame & $2.81(1.41)$ & .85 & $3.65(1.40)$ & .86 & $4.84^{* * *}$ \\
Face Concerns & $2.73(1.03)$ & .82 & $3.15(.94)$ & .65 & $3.41^{* * *}$ \\
Outgroup Trust & $4.52(1.12)$ & .81 & $3.12(1.02)$ & .72 & $-10.50^{* * *}$ \\
Empathy & $4.74(1.23)$ & .86 & $3.71(1.29)$ & .88 & $-6.66^{* * *}$ \\
Global Consciousness & $4.26(1.16)$ & .51 & $4.58(.79)$ & .83 & $2.65^{* *}$ \\
National Identity & $4.75(.86)$ & .87 & $4.48(.94)$ & .84 & $-2.46^{* *}$ \\
\hline Note. ${ }^{* *} p<.01, * * * p<.001$ & \multicolumn{5}{c}{} \\
\hline
\end{tabular}




\section{Distributional normality}

There were no serious deviations from normality since the skew values were below 1.0 and the kurtosis values were below 2.0 for each scale in Germany and Japan (Tabachnik \& Fidell, 2007).

\section{Structural equivalence}

I employed procrustean target rotation to test the structural equivalence of the newly devised scale "willingness to make amends". This analysis provides an estimate of the overall similarity after accounting for arbitrary sample-specific rotations (van de Vijver \& Leung, 1997). I conducted PCA with oblique rotation for each society separately to obtain the factor structure for each society. The factor loadings were then rotated via procrustean target rotation to make the factor structures maximally similar. I used the German factor solution as the norm and the factor structure of the Japanese data as the one that was rotated, for it is recommended if only two groups are involved (van de Vijver \& Leung, 1997).

The values for the factorial agreement (Tucker's Phi) were excellent. I received for the first factor (cognitive components of the willingness to make amends) a Tucker's Phi of .99 and for the second factor (behavioural components of the willingness to make amends) a Tucker's Phi of .97. Due to the high values of Tucker's Phi it is valid to assume that the content of the subscales had the same meaning for the samples from Germany and Japan.

I used multi-group $\mathrm{CFA}^{13}$ (see also Chapter 5) to test the structural equivalence for historical closure. The proposed one-factor structure fit well with the data $\left(\chi^{2}=4.7 ; \chi^{2} / \mathrm{df}=2.33, \mathrm{RMSEA}=.70 ; \mathrm{CFI}=.99\right)$. Therefore, the two

\footnotetext{
${ }^{13}$ The confirmatory approach was chosen, because the alternative - exploratory factor analysis and subsequent procrustean target rotation - is not applicable for one-factorial constructs.
} 
newly devised scales have similar underlying meaning across Germany and Japan and a comparison is justified.

Description of differences between Germany and Japan

Except for the outcome variable willingness to make amendments (cognitive component), there were significant differences on almost every scale (see Table 6.2), confirming hypothesis 1 . In the following section, I will describe the differences between Japan and Germany on the observed scales.

\section{Collective guilt, shame and face concerns}

Overall, independent t-tests revealed significant differences between Japan and Germany regarding the expression of guilt $(t(263)=7.39, p<.001)$ and shame $(t(263)=4.84, p<.001)$. Japanese participants expressed significantly more collective guilt $(M=4.28, S D=1.19)$ and shame $(M=3.64, S D=1.40)$ than German participants $\left(M_{\text {guilt }}=3.20, S D=1.19 ; M_{\text {shame }}=2.81, S D=1.41\right)$ for what their ancestors had done during WW II. This result does not confirm hypothesis 2. Furthermore, face concerns were quite low overall, but Japanese participants had significantly more face concerns than German participants had (see Table 6.2).

\section{Historical closure}

Perceiving the past as belonging to the past and not a source of interference with current interrelations was generally low (below the midpoint). However, German participants reported significantly more closure with the past than Japanese participants did. This can be interpreted in light of the current relations with former victimized nations for Germany and the burden of WW II as a vivid part of German identity (Hein \& Selden, 2000). Japan's reluctance to 
come to terms with her past and the ingrained victimhood in Japanese identity due to the atomic bombings may have contributed to the same low level of closure with the past. Low closure with the past should be associated with collective guilt and shame feelings.

\section{Perceived benefits of seeking forgiveness}

If benefits of seeking forgiveness are perceived as high, then this could potentially lead to a higher willingness to make amendments. German participants perceived significantly more benefits of seeking forgiveness than Japanese participants.

\section{Trust and empathy}

Trust in other Europeans was significantly higher among Germans compared to Japanese participants and their trust in other Asians. The same was the case for empathy or perspective taking: Germans were significantly more empathic towards other Europeans' feelings during WW II compared to Japanese participants and their expression of empathy towards other Asians during WW II. One open-ended question for each scale asked about which European nation or Asian nation respectively the participants thought of while answering.

When answering the trust questions Japanese participants thought the most of China (57.6\%), followed by the Korea (37.1\%), then the Philippines (10.6\%), Taiwan (5.3\%) and other Asian nations (18.9\%). German participants mentioned first France (41\%), then Poland (35.8\%), United Kingdom (27.6\%), followed by Russia (11.9\%). Other nations were mentioned second most (38.8\%) and other groups (Jews) were mentioned least $(0.7 \%)$ by German participants. 
The open-ended questions followed by the empathy scale were filled in as follows. Japanese participants mentioned China (52.3\%) the most, then Korea (34.1\%), followed by the Philippines (7.6\%) and Taiwan (5.3\%). Other Asian nations were mentioned to a proportion of $18.2 \%$ by Japanese participants. German participants thought of France the most (45.5\%), followed by Poland (39.6\%) and United Kingdom (27.6\%). Then other nations were mentioned $(27.6 \%)$ followed by Russia (15\%) and other groups (3\%). The percentages do not add up to a $100 \%$ since multiple answers were possible.

What is notable about the findings on trust, empathy and the corresponding open-ended answers is that overall Japanese participants reported a low sense of trust. Overall, German participants expressed significantly higher trust than Japanese did and most of the German participants mentioned France in the openended question. The same can be observed for empathy. Therefore, one could conclude that the trust and empathy ratings were lower in Japan, because the participants thought of China of which it is known that the relationships are tense due to the historical context of WW II. Consequently, Japanese participants were overly pessimistic, since they were thinking China rather than Taiwan or the Philippines.

It is generally accepted that Germany showed remorse for the harm done in the past and that Germany's relationship with the Allied nations first improved with France. France was mentioned the most among this German sample of university students in relation to outgroup trust and empathy. Therefore, German participants appeared overly optimistic, since they were thinking of France rather than Poland or Russia. 


\section{Willingness to make amendments}

As already reported, there were no differences between Germany and Japan on the cognitive component of the willingness to make amends $(t(262)=-$ $.14, p=.89$ ), but there were significant differences regarding the behavioural component $(t(262)=4.95, p<.001)$. Japanese participants were more willing to make behavioural amends $(M=2.97, S D=1.54)$ than Germans expressed $(M=$ $2.14, S D=1.17)$. The cognitive component of the willingness to make amends was above the neutral 4 point $\left(M_{\text {Germany }}=4.68, S D=1.37 ; M_{\text {Japan }}=4.70, S D=\right.$ 1.49) and the behavioural component of the willingness to make amends was below the neutral point. In general, the expression of the behavioural component was seemingly lower than the cognitive component among both samples confirming hypothesis 3 .

Identity

Surprisingly, Germans in this study expressed relatively high levels of national identity, which was significantly higher than that of Japanese participants. In general, the levels of national identity were quite high among both Germans and Japanese participants compared to previous findings (Atsumi, Suwa, Kobayahi, Miyamoto, \& Seki, in press; Hilton \& Liu, 2008). The correlation between national identity and assessed other constructs revealed different patterns for Japan and Germany. Among the Japanese sample national identification was positively associated with collective shame, behavioural components and cognitive components of the willingness to make amends whereas among the German sample national identification was not associated with either guilt or shame feelings, but negatively with behavioural components and cognitive 
components of the willingness to make amends and positively with historical closure (see Table 6.3).

The finding regarding the willingness to make amends is surprising since it is argued in literature that individuals identifying strongly with their nation have a strong motivation to maintain a positive social identity (Turner, 1999). This motivation leads to a tendency to distance oneself from harm done in the past, leading to a reluctance to make amends (Branscombe \& Doosje, 2004). Therefore, a quite intriguing finding is this reversed effect in the Japanese sample. The results delivered some qualified support for hypothesis 4 in the German sample and poor support for the Japanese sample regarding the national identification part of the hypothesis.

\section{Global consciousness}

Global consciousness was higher among Japanese participants than among German participants. In each sample global consciousness was seemingly lower than national identity. The correlations showed similar patterns. Global consciousness was positively associated with behavioural components and cognitive components of the willingness to make amends, collective guilt, and collective shame (not significant in Germany), outgroup trust, empathy and negatively with historical closure and face concerns (see Table 6.3). This confirms the second part of hypothesis 4 . There is a general pattern. Participants who were globally more conscious experienced more collective guilt and shame compared to those with low global consciousness. 


\section{Interrelationships between concepts}

To answer the research question, I first investigated the relationships and then the contribution of the concepts to predict the willingness to make amends. I explored the interrelationships between assessed concepts and the outcome variables willingness to make amends (behavioural and cognitive component), and concepts introduced in Chapter 5 (historical closure) and newly devised concepts (face concerns, global consciousness and benefits of seeking forgiveness). For this purpose, I conducted Pearsons-Product-Moment correlations for each sample separately (see Table 6.3). I found similar correlational patterns across the two samples, but also some striking differences that deserve more attention.

\section{Japan versus Germany}

In Japan, the cognitive component of the willingness to make amends was significantly positively associated with benefits of seeking forgiveness, collective guilt, collective shame, outgroup trust, empathy, global consciousness and national identity and significantly negatively related to historical closure and face concerns. In Germany, the associations between willingness to make amends and the other concepts were similar, except for national identity, which was negatively related to the cognitive component of making amends. Regarding the behavioural component of willingness to make amends, there were significant positive correlations with benefits of seeking forgiveness, collective guilt, collective shame, outgroup trust, empathy, face concerns, global consciousness and face concerns, whereas there was a significant positive association with historical closure in Japan. I found the same difference in Germany with the cognitive 
component, whilst national identity was negatively related to the behavioural component.

Historical closure was significantly negatively related to the benefits of seeking forgiveness, collective guilt, collective shame, outgroup trust, empathy and global consciousness and significant positively related to face concern in the Japanese sample. In Germany, the pattern was similar, but additionally national identity was positively related to historical closure.

Face concerns, an Asian concept, assessed as the fear of losing face caused by the responsiveness of the government due to the harm done during WW II by Japan was negatively associated with outgroup trust, empathy, collective guilt and shame, benefits of seeking forgiveness and global consciousness and positively with national identification and historical closure in Japan. In Germany, negative correlations were only found for trust, empathy, benefits of seeking forgiveness and global consciousness and a positive association with historical closure.

In Japan, the perception of the benefits of seeking forgiveness was significantly negatively associated with face concerns. Positive associations were found between the benefits of seeking forgiveness and guilt, shame, trust and empathy. In Germany, the group-based emotions guilt and shame did not correlate with the benefits of seeking forgiveness, but trust and empathy as well as global consciousness were significantly positively related to this perceived benefit.

The identification with a more inclusive level of identity, namely being globally conscious, was significantly positively associated with guilt, shame, trust and empathy in Japan. Significant negative relationships were found with historical closure and face concerns. In Germany, national identity was negatively 
related to global consciousness. The other correlations were quite similar except for shame which had no significant relationship with these variables.

\section{Similarity of correlation coefficients}

In the following analysis, a z-test of r-to-z transformed correlation coefficients examined the similarity of correlation coefficients between Japan and Germany. The focus was on the role of identification and the role of historical closure, testing the similarity will reveal whether correlations are truly different and not due to fluctuation.

There were significant differences between Japan and Germany for following associations: national identity and collective guilt $(z=2.45, p<.01)$, collective shame $(z=1.84, p<.05)$, the cognitive component of the willingness to make amends $(z=4.48, p<.001)$, the behavioural component $(z=5.97, p<.001)$, historical closure $(z=-3.33, p<.001)$, and global consciousness $(z=3.20, p<$ .001). There was no significant difference between Japan and Germany for the association between national identity and face concerns $(z=1.33, p=.09)$.

There was only one significant difference between Japan and Germany for the association between historical closure and collective guilt $(z=-2.81, p<.01)$. All other correlation coefficients for historical closure were similar between Japan and Germany pointing to a meaningful general pattern of associations.

In sum, the most intriguing differences in the correlational pattern across these two contexts were the role of national identity and of group-based emotions (guilt and shame). These seem to have different functions across the two contexts that may be due to the different historical context. The correlation between collective guilt and shame were significantly positive, which is in line with previous research in both contexts. 
Table 6.3

Intercorrelation Matrix for all scales in Japan $(N=132)$ and Germany $(N=134)(\text { Study } 4)^{14}$

\begin{tabular}{|c|c|c|c|c|c|c|c|c|c|c|c|}
\hline Scale & 1 & 2 & 3 & 4 & 5 & 6 & 7 & 8 & 9 & 10 & 11 \\
\hline 1. Amendments (cognitive) & - & $.39 * * *$ & $-.46 * * *$ & $.29 * * *$ & $.36 * * *$ & $.31 * * *$ & $-.38 * * *$ & $.31 * * *$ & $.46 * * *$ & $.49 * * *$ & $-.21 * *$ \\
\hline 2. Amendments (behaviour) & $.45 * * *$ & - & $-.31 * * *$ & .10 & $.56 * * *$ & $.54 * * *$ & .07 & $.31 * * *$ & $.28 * * *$ & $.31 * * *$ & $-.25 * *$ \\
\hline 3. Historical Closure & $-.44 * * *$ & $-.39 * * *$ & - & -.11 & $-.29 * * *$ & $-.27 * * *$ & $.32 * * *$ & -.12 & $-.33 * * *$ & $-.37 * * *$ & $.33 * * *$ \\
\hline 4. Benefits of seeking forgiveness & $.31 * * *$ & $.21^{*}$ & $-.24 * *$ & - & .11 & .06 & $-.25 * *$ & $.30 * * *$ & $.22 * *$ & $.27 * *$ & -.07 \\
\hline 5. Guilt & $.51 * * *$ & $.41 * * *$ & $-.57 * * *$ & $.26 * *$ & - & $.69 * * *$ & -.16 & $.28 * * *$ & $.41 * * *$ & $.25 * *$ & -.08 \\
\hline 6. Shame & $.30 * * *$ & $.30 * * *$ & $-.36 * * *$ & $.26 * *$ & $.62 * * *$ & 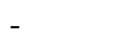 & -.04 & $.21 * *$ & $.34 * * *$ & .13 & -.13 \\
\hline 7. Face Concerns & $-.18 *$ & -.004 & $.33 * * *$ & $-.28 * *$ & $-.25 * *$ & -.14 & - & $-.24 * *$ & $-.28 * * *$ & $-.35 * * *$ & .09 \\
\hline 8. Outgroup Trust & $.39 * * *$ & $.23 * *$ & $-.20 *$ & $.29 * * *$ & $.38 * * *$ & $.29 * * *$ & $-.17 *$ & - & $.30 * * *$ & $.35 * * *$ & -.13 \\
\hline 9. Empathy & $.41 * * *$ & $.42 * * *$ & $-.41 * * *$ & $.24 * *$ & $.56^{* * *}$ & $.33 * * *$ & -.15 & $-.38 * * *$ & - & $.37 * * *$ & -.05 \\
\hline 10. Global Consciousness & $.28 * * *$ & $.25 * *$ & $-.25 * *$ & .15 & $.29 * * *$ & $.23 * *$ & $-.33 * * *$ & $.22 * *$ & $.35 * * *$ & - & $-.46 * * *$ \\
\hline 11. National Identity & $.33 * *$ & $.18^{*}$ & -.07 & .03 & $.22 *$ & .11 & $.25^{* *}$ & .10 & .16 & -.10 & - \\
\hline
\end{tabular}

Note. German values are above the diagonal; Japanese values below the diagonal.

$* p<.05, * * p<.01, * * * p<.001$, two-tailed

\footnotetext{
${ }^{14}$ There might be similar content in some items measuring guilt and the willingness to make amends items. In order to explore potential conceptual overlap, I conducted PCA with oblique rotation. I received a three factor structure (cognitive amends, behavioral amends and guilt) explaining $74.6 \%$ of the variance. Only one guilt item ("I feel regret for Japan's past harmful actions towards other Asian nations") had cross-loadings with the cognitive amends factor. All other guilt items formed a distinct factor. I re-analyzed the data without the cross-loaded guilt item and the results remained the same.
} 


\section{Multiple Hierarchical Regression}

Multiple hierarchical regressions tested the contribution of established (such as collective guilt, empathy and newly devised scales (such as historical closure) to explain variance in the willingness to make amends (cognitive and behavioural) separately for the German and Japanese contexts (see Table 6.4 - Table 6.5). Separate regressions were conducted in order to investigate differences between the two contexts and to reveal the unique contribution of new concepts added last in the equation. Since there were gender and age differences on some scales, I controlled for gender and age effects and entered them first in the equation. Established predictors were entered in Step 2. The variance inflation factor (VIF) was below 3, indicating no problems with multicollinearity.

\section{Cognitive component of the willingness to make amendments}

The first Step revealed gender differences in both contexts, but this effect only explained unique variance in the Japanese sample. In this case, female participants were more willing to make amends than male participants were. However, the gender effect disappeared in Step 2. Therefore, the gender differences were explained by the other entered variables. There were differences in predicting willingness to make amends in Step 2 between Germany and Japan. Among the German sample only empathy was a positive predictor for the willingness to make amends, whereas among the Japanese sample collective guilt, outgroup trust and national identity were positive contributors. In Step 3, historical closure was a significant negative and global consciousness a significant positive predictor for willingness to make amends in the German sample, whereas in the Japanese sample only historical closure predicted significant variance in cognitive willingness to make amends. Historical closure significantly contributed to 
explaining variance in the cognitive component of the willingness to make amends in both contexts (see Table 6.4).

Testing for homogeneity of the regression coefficients for cognitive component

Furthermore, I tested the homogeneity of the regression weights with each predictor using MANOVA as suggested by Tabachnik and Fidell (2007). The test of homogeneity of regression coefficients has the purpose to test whether the linear relationship between two concepts are similar between the samples. This indicates that (theoretically) the underlying process is the same in the samples from different countries. If the regression coefficients are homogenous, it can be assumed that the process is theoretically similar. If they are heterogeneous, then there are different processes taking place. This was the case for national identity. This is evident in the opposite effect of national identity to predict the willingness to make amends in the regression for the Japanese sample.

Table 6.4

German versus Japanese context willingness to make amends (cognitive component), standardized $\beta$-weights (Study 4)

\begin{tabular}{|c|c|c|c|c|c|c|}
\hline \multirow[t]{2}{*}{ Model } & \multicolumn{2}{|l|}{ Step 1} & \multicolumn{2}{|l|}{ Step 2} & \multicolumn{2}{|l|}{ Step 3} \\
\hline & Germany $^{\mathrm{a}}$ & Japan $^{b}$ & Germany & Japan & Germany & Japan \\
\hline 1. gender & $.17 *$ & $.25 * *$ & .04 & .13 & -.05 & .08 \\
\hline age & .10 & .06 & .04 & .02 & -.02 & -.02 \\
\hline 2. guilt & & & .13 & $.35^{* *}$ & .03 & .23 \\
\hline shame & & & .07 & -.04 & .12 & -.06 \\
\hline trust & & & .13 & $.21 *$ & .05 & .15 \\
\hline empathy & & & $.33 * * *$ & .07 & $.17 *$ & .02 \\
\hline national identity & & & -.14 & $.21 * *$ & .02 & $.26 * * *$ \\
\hline 3. face concerns & & & & & -.13 & -.04 \\
\hline benefits seeking & & & & & & \\
\hline forgiveness & & & & & .10 & .15 \\
\hline historical closure & & & & & $-.23 * *$ & $-.24 * *$ \\
\hline global consciousness & & & & & $.25 * *$ & .14 \\
\hline $\begin{array}{l}\text { Note. a. } R^{2}=.02 \text { for Step } 1 \text {, } \\
\text { Adjusted } R^{2}=.39 \text { for Step } \\
\text { b. } R^{2}=.05 \text { for Step } 1, \Delta R^{2} \\
\text { Adjusted } R^{2}=.42 \text { for Step } 3 \\
* p<.05 ; * * p<.01 ; * * * p\end{array}$ & $\begin{array}{l}R^{2}=.04(p= \\
\Delta R^{2}=.14(p \\
07(p<.05) \\
\Delta R^{2}=.09(p \\
.001\end{array}$ & $\begin{array}{l}.09), A d \\
.001) \\
\text { Adjustec } \\
.01)\end{array}$ & $\begin{array}{l}d R^{2}=.2 \\
=.35 \text { for }\end{array}$ & $\begin{array}{l}\text { Step } \\
2, \Delta I\end{array}$ & $\begin{array}{l}R^{2}=.26(p \\
32(p<.00\end{array}$ & .001), \\
\hline
\end{tabular}


Behavioural component of the willingness to make amends

Here as well, the first Step showed significant gender differences, but only in the German sample, and this step did not explain significant variance. Gender effects disappeared in Step 2. There were differences in the prediction of willingness to make amends in Step 2 between Germany and Japan. Among the German sample group-based emotions (guilt and shame) were the only positive predictor for the willingness to make amends at the behavioural level, whereas among the Japanese sample empathy was the only positive predictor. In Step 3, only face concerns was a significant positive predictor for willingness to make amends in the German sample, whereas in the Japanese sample face concerns and historical closure predicted significant variance in the willingness to make amends at the behavioural level (see Table 6.5).

Testing for homogeneity of the regression coefficients for behavioural component

I used the same procedure as aforementioned and the only heterogeneous regression coefficient was national identity. All other tested regression weights were homogenous. Therefore, the underlying process is different for national identity. 
Table 6.5 German versus Japanese context predicting willingness to make amends (behavioural component), standardized $\beta$-weights (Study 4)

\begin{tabular}{|c|c|c|c|c|c|c|}
\hline \multirow[t]{2}{*}{ Model } & \multicolumn{2}{|l|}{ Step 1} & \multicolumn{2}{|l|}{ Step 2} & \multicolumn{2}{|l|}{ Step 3} \\
\hline & Germany $^{\mathrm{a}}$ & Japan $^{\mathrm{b}}$ & Germany & Japan & Germany & Japan \\
\hline 1. gender & $.25 *$ & .15 & .10 & .03 & .07 & -.01 \\
\hline age & .04 & .10 & .13 & .05 & .12 & .01 \\
\hline 2. guilt & & & $.33 * *$ & .12 & $.32 * * *$ & .02 \\
\hline shame & & & $.30 * *$ & .07 & $.27 * *$ & .03 \\
\hline trust & & & .12 & .05 & .14 & .06 \\
\hline empathy & & & -.03 & $.32 * *$ & -.03 & $.22 *$ \\
\hline national identity & & & -.12 & .11 & -.06 & .10 \\
\hline $\begin{array}{l}\text { 3. face concerns } \\
\text { benefits seeking }\end{array}$ & & & & & $.27 * * *$ & $.20 *$ \\
\hline forgiveness & & & & & .02 & .02 \\
\hline historical closure & & & & & -.12 & $-.34 * * *$ \\
\hline global consciousness & & & & & .12 & .14 \\
\hline $\begin{array}{l}\text { Note. a. } R^{2}=.05 \text { for Step } 1 \text {, } \\
\text { Adjusted } R^{2}=.45 \text { for Step } 3 \\
\text { b. Adjusted } R^{2}=.02 \text { for Ste } \\
.001), \text { Adjusted } R^{2}=.30 \text { for } \\
* p<.05 ; * * p<.01 ; * * * p\end{array}$ & $\begin{array}{l}\Delta R^{2}=.06(p \\
\Delta R^{2}=.06(p \\
1, \Delta R^{2}=.04 \\
\text { Step 3, } \Delta R^{2}= \\
.001\end{array}$ & $\begin{array}{l}.05), A c \\
.01) ; \\
p=.13) \\
10(p<\end{array}$ & $\begin{array}{l}\text { ted } R^{2}=.4( \\
\text { djusted } R^{2} \\
) ;\end{array}$ & $\begin{array}{l}\text { or Step } \\
22 \text { for } S\end{array}$ & $\begin{array}{l}R^{2}=.37(p \\
2, \Delta R^{2}=.2\end{array}$ & $\begin{array}{l}.001), \\
(p<\end{array}$ \\
\hline
\end{tabular}

In summary, differences were found between the Japanese and German samples for the prediction of the cognitive and behavioural components of the willingness to make amends. Although gender differences emerged initially, these were explained in the subsequent steps of the hierarchical regression and the effect disappeared. Apparently, Japanese participants reporting high collective guilt feelings, high outgroup trust and high national identification were more willing to make amends on the cognitive component, whereas German participants who empathized with Germany's WW II victims more strongly reported more willingness to make amends on this component. However, in both contexts those effects weakened or disappeared completely (see Japanese sample, Step 3) when historical closure and other new variables were entered. For the cognitive component, historical closure seemed to be a key predictor along with global consciousness in the German sample. Benefits of seeking forgiveness and face concerns however remained insignificant. The last step 
explained a significant amount of unique variance in both contexts (Germany: $\Delta R^{2}=$ $.14, F_{\text {change }}(4,121)=7.77, p<.001 ;$ Japan: $\left.\Delta R^{2}=.09, F_{\text {change }}(4,108)=4.35, p<.01\right)$.

The behavioural component of willingness to make amends at the individual level (getting actively involved in showing support) was governed by feelings of collective guilt and shame in the German sample, whereas in the Japanese sample empathy was a significant positive predictor. Here as well, the effects in Step 2 became weaker when variables were added in Step 3. Face concerns were the only significant positive predictor in Step 3 in the German sample. Therefore, the more German participants reported concerns about losing face due to government actions due to WW II, the more German participant were willing to take personal actions to show support $\left(\Delta R^{2}=.06 ; F_{\text {change }}(4,121)=3.76, p<.01\right)$. In the Japanese sample, historical closure and additionally face concerns explained variation in this component of willingness to make amendments explaining $10 \%$ of unique proportion of the variance $\left(F_{\text {change }}(4,108)\right.$ $=4.37, p<.01)$.

\section{Discussion}

The results supported hypothesis 1 . There were mean differences on virtually all concepts between Germany and Japan. These differences can be potentially explained by the historical context. The answers to the open-ended questions for the trust and empathy scales were especially revealing. China and Japan have a relationship that is heavily burdened by the harm committed by Japan during WW II and the Sino-Japanese War. This is evident in recent outbursts of outrage by China for Japan's desire to obtain a permanent seat for the UN and a strong Chinese historical redress movement (Reilly, 2006). Although there is movement in Japan called the "Association for liberal view of history" which is a revisionist movement, there is also an opposing movement within 
Japan consisting of teacher's unions. Furthermore, the influence of the dissemination of information and debates via the internet should not be underestimated (Morris-Suzuki \& Rimmer, 2002). Therefore, it is arguable that a sample of university students is likely to be well aware of the problems between former victimized East Asian nations and Japan. Arguably, the fact that nothing or little is done to challenge the revisionist opinions may be one reason why Japanese students feel collectively guilty and have low closure with the past.

Hypothesis 2 was not confirmed. Based on the claim made by anthropologists (e.g. Benedict, 1946; Creighton, 1990) that Japan is a so-called "shame" culture and Germany a so-called "guilt" culture, I expected Japanese participants to experience more shame feelings than German participants. This was not the case. Japanese participants reported more collective guilt feelings and less shame feelings than German participants. Since newer generations of Japanese people have access to diverse international debates due to Japan's handling of the past misdeeds via multiple media outlets (Morris-Suzuki \& Rimmer, 2002), the likelihood is quite high that the Japanese participants have come across opposing opinions and had to deal with a portrayal as aggressors during WW II (Penney, 2008). This could have evoked bad feelings on behalf of their nation, since it is contradicting what the Japanese government represents regarding their history and what is publicly and internationally debated. Furthermore, staunch positions held by some parts of the Japan's political elites cause resentment in other East Asian countries. This could be prevented and may be controllable by taking symbolic actions, such as the visit of a memorial commemorating victims other than Japanese by a highly respected representative of Japan. Thus, Japanese participants experienced more guilt than shame feelings. 
Hypothesis 3 was supported. The cognitive component was higher than the behavioural component of the willingness to make amends confirming previous assumptions about the difficulty to change the past and therefore preferring cognitive responses over behavioural ones. Although the behavioural items were phrased in a way that the actions described could be done today, they still do not change the past.

Hypothesis 4 was only partially supported in Germany and poorly supported in Japan. National identification was not related to guilt and shame, but to historical closure, global consciousness and making amends in Germany. This is partially in line with SIT/SCT. The more Germans identified with their nation, the less willingness they had to make amends, the less they identified with a global identity, and the more they reported closure with the past. National identification did not contribute to the prediction of willingness to make amends for both components.

For the Japanese sample, national identification was related to guilt, face concerns and to both components of the willingness to make amends. The correlations with guilt and willingness to make amends were not in line with SIT/SCT since the relationships were positive: the higher Japanese identified with their nation, the more they felt guilty and were more willing to make amends. This is a very surprising result, and should be investigated more thoroughly in future research. National identity as a form of collective identity was a positive contributor to predict willingness to make amends, but only for the cognitive component. In both contexts, the more the participants were globally conscious, the more collectively guilty they felt and were less concerned about the loss of face; only in Japan, participants also felt collectively ashamed. 
It could be argued that gender effects drove the directions of effects since the distribution of males and females was unbalanced. Indeed, females and males differed significantly on some of the assessed constructs. For instance, females had less closure with the past and experienced more guilt than males did when tested via independent ttests in Japan. In Germany, the differences were smaller and for the most part nonsignificant. For Japan, the gender differences can be also explained by the historical context, since one of the most prominent example of Japan's past harm done is the Comfort Women issue. Therefore, female participants were more likely to empathise with former female victims of Japan's war of aggression. However, the gender effects disappeared when regression models were tested. Nevertheless, gender effects should be taken more into considerations in future research and a balanced gender distribution is helpful to rule out gender as driving the effects.

In order to answer the research question regarding the unique contribution of the new concepts, I conducted multiple hierarchical regressions. Historical closure significantly contributed to explain variance in willingness to make amends (cognitive). The lower closure was, the more willingness to make amends was reported. In Germany, global consciousness was also among the significant predictors. In both contexts, established predictors (such as collective guilt) got weaker or their contribution disappeared completely, pointing to the importance of historical closure for the overall process, which was already shown to be an important concept in Study 3. Face concerns were a significant contributor in both contexts to predict the behavioural component of willingness to make amends. Only in Japan did historical closure also explain unique variance. Historical closure or in this study the lack of historical closure 
showed similar patterns for predicting the cognitive component of the willingness to make amends.

One question is why identity did not explain variation in willingness to make amends at all in the German context, even though it has been continuously emphasized and empirically demonstrated that intergroup conflict are issues involving identity (e.g. Noor et al., 2008). Another question is also why identity is a positive predictor in the Japanese context for the willingness to make amends. The role of national identification in Japan seems contradictory, since high identification with one's nation that has dark parts in its history should lead to a distancing process and eventually in a denial of guilt to enable a person to maintain a positive social identity.

However, Gries and Peng (2002) argued that "Western reasoning tends to focus on objects and categories, and is driven by formal logic; in the East, by contrast, reasoning embraces contradictions among objects in a yin-yang field of constant change" (p. 175). Therefore, to be highly identified with one's nations and experiencing collective guilt for past misdeeds are not necessarily a contradiction in an East Asian context. On the contrary, it might be an expression of showing loyalty to care about the standing of one's nation in being honest about the past. Therefore, the historical context seems to be a key factor to explain the differences between Germany and Japan. 


\title{
Chapter 7
}

\section{General Discussion and Conclusions}

\begin{abstract}
Summary
This thesis' overall aim was to extend existing research on intergroup forgiveness and to shed light on the complex process of intergroup forgiveness between nations in the post WW II context. In four studies, the role of the societal and historical context was taken into consideration from different analytical angles and used as sources for predictions and explanations of intergroup forgiveness.

To reiterate, Study 1 was a cross-national meta-analytical approach that aimed to explain differences in interpersonal forgiveness based on contextual variables. This study discovered differences in interpersonal forgiveness between the 13 societies that were included in the meta-analysis. The inclusion of contextual variables delivered opportunities to explain these differences. That is, socio-economic and socio-political contextual variables, societal peacefulness, well-being and more negative evaluations of historical calamities were significantly associated with interpersonal forgiveness. Furthermore, in conflict-prone societies, interpersonal forgiveness was lower than in societies in which there was no apparent conflict potential.

Study 2 revealed insights into the articulation and conceptualisation of intergroup forgiveness and crucial contextual factors by giving a qualitative snapshot of the social reality of participants coming from three different countries (Germany, Japan, the Philippines). This qualitative approach revealed an important context relevant concept, which was coined as historical closure and was conceptualized as an attitude which content was drawn from the historical context of harm committed during WW II.
\end{abstract}


This concept (among others) was devised as a scale and applied in the subsequent Studies 3 and 4.

Study 3 was devoted to scrutinize the unique contribution of (a) new concepts and (b) contextual variables on the willingness to forgive in samples of six formerly victimized WW II nations. Furthermore, the study explored the differences in intergroup forgiveness and in psychological antecedents of intergroup forgiveness across two post WW II contexts: Europe and East Asia. This study revealed insights into the role of historical closure and the costs of granting forgiveness. These contextualized variables contributed to predict intergroup forgiveness in samples from formerly victimized nations, explained unique variance in intergroup forgiveness, and weakened contributions of established predictors (such as guilt). Furthermore, identity neither predicted intergroup forgiveness directly nor mediated relationships as expected. Additionally, a novel approach using a cross-level operator analysis was applied to investigate the impact of historical context variables. This analysis showed that the number of official apologies contributed to explain variations in intergroup forgiveness.

The emphasis of Study 4 was on the perpetrator perspective, examining the impact of historical closure among other psychological concepts on the willingness to make amendments. Here as well, the concept of historical closure contributed significantly to predict different components of the willingness to make amends. Furthermore, participants identifying more strongly with their nation did not experience less guilt or shame feelings compared to those identifying not as strong with their nation. Additionally, national identification did not contribute to explain variance in the willingness to make amends. On the other hand, participants being more globally conscious experienced more collective guilt and shame than those who were low. These 
results contribute to a more holistic understanding about the overall intergroup forgiveness process, since both victim and perpetrator perspectives were taken into consideration.

\section{Contributions of this thesis}

This thesis contributes to the intergroup forgiveness literature in several ways. Primarily, this thesis examined the post WW II context and compared participants from three formerly victimized European nations (France, Poland, Russia) and from three formerly victimized East Asian nations (China, Taiwan, the Philippines). Furthermore, the perspectives from formerly perpetrating nations were captured comparing samples from Germany and Japan. In the post WW II context, there are clear victim and perpetrator roles. As these countries do not share a region and its citizens live in their own countries, this thesis examined between-societies situations.

This thesis goes beyond previous research involving groups sharing one geographical region in which the victim and perpetrator roles were interwoven. I examined one specific context, that is the post WW II relations between formerly victimized and victimizer societies. Furthermore, I investigated within the setting of post WW II two distinct contexts: the European and the East Asian. In addition, victim and perpetrator perspectives in Europe were compared to those in East Asia. Therefore, this thesis allowed gaining insights into the intergroup forgiveness process. It examined people's experiences of group-based emotions, closure with the past, and intergroup forgiveness determined by the historical context. The participants belonged to the third generations after WW II. Although the end of WW II is almost 70 years ago and the participants neither experienced nor committed directly harm, this thesis demonstrated 
that specifically in the Sino-Japanese context resentment and grievance can last for generations.

The application of a cross-national meta-analytical approach in Study 1 allowed the contribution of contextual variables to explain differences in interpersonal forgiveness to be assessed across 13 societies. This analysis revealed that contextual variables mattered and influenced variations in interpersonal forgiveness. The role of context had previously been under researched and this cross-national meta-analysis demonstrated that context should be considered. Additionally, this led to the question to what extent contextual variables could explain variation at the intergroup level, considering the complexity of context in an intergroup situation as opposed to a conflict between two individuals.

Furthermore, the approach to consider the historical context for further predictions and explanations regarding intergroup forgiveness is a novel and important contribution to the literature. Since East Asian perspectives have been continuously neglected, this thesis contributes to a better understanding of the intergroup forgiveness process in East Asian settings. An important discovery is historical closure defined as an attitudinal content drawn from the historical context. Historical closure reflects the extent that the past is perceived to still affect the present and future, and what implications it has for present-day relationships between groups (see Chapter 5).

It seemed that closure (or lack of) with the past is more important than identifying with one's nation in the European and East Asian context and for both subject positions. It can be speculated that in France closure was achieved signalling a departure from the past that allowed promotion of forgiveness. However, the German participants had relatively low closure with the past. How does this fit? One reason 
could be that even today's Germans carry the burden of the dark parts of their history. Having closure with one's past could be misunderstood as ignoring the past. Germany's post war motto of "nie wieder Krieg" (no more war) indicates that closure can only be achieved, if it is balanced with adequate remembrance and acknowledgment. Furthermore, a particular lack of closure for former perpetrators seems important in order to be supportive in terms of compensation claims. This may apply in similar ways to the Japanese context. Additionally, perceived costs of granting forgiveness was identified as an obstacle to the willingness to forgive Japan and Germany respectively. The application of this new concepts explained variance beyond established psychological contributors such as guilt. However, the new concept or the analyses posit further questions.

\section{Theoretical implications of the current thesis}

\section{Does context matter?}

From a theoretical stance, the findings from the cross-national meta-analysis support Inglehart's (1997) propositions about post-materialism and Fukuyama's (1992) liberal democracy argument. Inglehart (1997) claimed that socio-economic development (affluence of a society) drives the shift from materialistic to post-materialistic values which affects newer generations that are more socially and economically secure than older generations. Fukuyama (1992) proposed that societies eventually will move towards liberal democracies, which may lead to decreased warfare, and less internal and external political violence.

These two theories complement each other, since socio-economic security and human development indicating a fulfilment of survival needs have been linked to peace 
(Fischer \& Hanke, 2009). Peace is linked to decreased warfare and according to Fukuyama (1992) to liberal democracies. The two theories also complement the metaanalysis results, since there was a linear relationship between socio-economic and sociopolitical correlates, the peacefulness of societies, evaluations of historical calamities and interpersonal forgiveness. Does this imply that the more a society is able to provide a safe environment, that it will eventually enhance the likelihood of the implementation of forgiveness practices? Does this mean that, for example when China gains more economic power and becomes more wealthy, this will translate into more civil liberties, less internal and external violence and more implementation of forgiveness practices which are related to peace building?

Assuming the multidimensionality of forgiveness (Worthington, 2005), in which many factors come into play, it seems fair to argue against this notion. These economic and democratic factors play a role, but other factors may be equally important, making forgiveness a multidimensional rather than a one-dimensional concept. One hint can be gleaned from the association between the evaluations of negative historical events and interpersonal forgiveness. Though probably not completely independent from socioeconomic and socio-political factors, evaluations of historical events add another dimension to interpersonal forgiveness.

Since forgiveness is a moral response option after a transgression, evaluating human tragedies during the course of history as negative may lead to consider alternatives - other than holding grudges or getting even - to resolve conflicts. Hence, if a society is aware that calamities are generally negative, (which is not always the case, since other societies have benefited from war), it probably helps encouraging the more prosocial response of forgiving after transgressions. It may be doubtful that this process 
is only explainable through socio-economic security. Notwithstanding, correlational data should always be interpreted with caution and assurances about their causal implications can only be drawn through longitudinal designs.

The context in the current thesis is post WW II. Social representations of history and identity (Hilton \& Liu, 2008; Liu \& Hilton, 2005) proposes that how history is represented and how knowledge about it is transferred can be done purposely in order to follow a specific agenda (e.g. to mobilize particular opinions in a context). Especially, diversity of social representations of WW II and its aftermath between China and Japan may induce intolerance or disregard towards the other's accounts and reduce empathic positions. Eventually, this may lead to conflicts on a symbolic level and ill feelings about how the others portray their "truth" evoking irreconcilable positions and reducing the willingness to be forgiving. This is particular evident for the Sino-Japanese case, since for Japanese and Chinese participants closure with the past was relatively low and Chinese participants were quite unforgiving.

In support of this assumption were the significant differences between the six societies, for the most part carried by differences between China and France. However, in many cases Filipinos and Taiwanese were also different from Russians and Poles. Filipino and Taiwanese participants assigned more guilt, were angrier and were slightly less forgiving than Russians and Poles. Yet, Filipinos and Taiwanese had more closure with the past than Russians and Poles. Therefore, in the European context the participants were more positive towards Germany, since they expressed less guilt assignment, less anger and were more forgiving than in the East Asian context. The most opposing samples were from France and China in terms of their expressions of guilt assignment, anger, and forgiveness. 
These findings suggest that there is a greater shared understanding of the past between France and Germany, pointing to more shared interpretations of WW II. The French data in particular portrays this since French participants were the most forgiving and had the highest closure with the past. This appears to reflect the efforts made by France and Germany since 1951 for rapprochement and the development of a shared representation of WW II in form of textbooks. In contrast, in the East Asian context and especially the Chinese participants - were less forgiving and expressed more anger and guilt assignment than France and the other East Asian countries, pointing to more polemical representations between Japan and China.

Since it is no secret that Japan and China have a difficult relationship which can be traced back to unresolved issues due to WW II and the Sino-Japanese War, controversies and debates over the past are evident (e.g. Raudsepp, 2005). The lack of a shared understanding of the past is associated with large mean differences between China (high on guilt assignment, high on anger, low on trust, low on closure, low on forgiveness) and France (low on guilt assignment, low on anger, high on trust, high on closure, high on forgiveness).

The Filipino and Taiwanese sample were relatively similar in their report of historical closure and other variables, and were less negative towards Japan (e.g. less guilt assignment and anger than Chinese participants). One explanation can be contextual. Taiwan benefited in terms of infrastructure and education from the Japanese colonisation period, which is still evident in the display of Japanese culture in Taiwan. The Philippines is in a less powerful position than China, making it difficult to speak-up against past injustices, since the Philippines are dependent in many ways on other countries' economic help - including Japan. A complementary or alternative 
explanation why Chinese participants were quite unforgiving is the fact that there are conflicting representations of WW II and the atrocities committed against China by Japan that are linked to these ill feelings about the past (Liu \& Atsumi, 2008).

The Polish and Russian participants delivered a different picture. Poles and Russians were similar in their degree of guilt assignment and trust. However, Russians experienced more anger, perceived more costs associated with forgiveness, were less forgiving, but had more closure with the past than Poles. One reason for this result could be recent political tensions between Germany and Russia, which may have resulted in the mobilisation of the past to encourage resentment towards Germany.

Another theoretical explanation for the overall pattern may be the reasoning behind high versus low-context cultures (Hall, 1976). In high-context cultures like China and Japan communication and negotiation processes are based on the physical context or internalised meanings (Rosenberg, 2004). In these processes, little or no communication is prevalent probably resulting in different expectations (that are not met in the context of post WW II relations between China and Japan) based on different internalised interpretations of the past. Germany and France are low-context cultures meaning that their communication is much more explicit which probably has lead to the early explicit exchange evident in textbook commissions.

Further empirical evidence supports these theoretical explanations, since the cross-level analysis revealed that the number of official apologies during the last two decades was a negative predictor for intergroup forgiveness. This result points to the explicit character of a verbally delivered apology probably not being helpful, since it does not change the fact that parts of the Japanese political elites are repeatedly downplaying Japan's aggressor role during WW II. It seems apologies are issued 
because it is internationally demanded especially by the US, but these apologies may not be perceived as sincere. The idiom "actions speak louder than words" may be taken literally. Independent from the reasoning about high versus low-context cultures, in the German context this literal meaning of the aforementioned saying was demonstrated by Willy Brandt's silent kneeling in front of the Warsaw memorial commemorating Jewish victims of the Holocaust that opened up the possibility for rapprochements between Germany and Eastern Europe. It is possible that Chinese expect something similar from Japan.

\section{Predictors of intergroup forgiveness and willingness to make amends}

Study 2 explored the way that forgiveness is perceived as a means to resolve conflict at the individual level and how the differences between interpersonal and intergroup forgiveness are articulated. In this qualitative study, interviews revealed that forgiveness may not be readily applied in a group setting, since it is not thought of to be crucially important among the Japanese interviewees. Additionally, one reason for difficulties to apply forgiveness in group settings was that most interviewees seemed to be more used to conceiving forgiveness relevant at the individual level. Furthermore, among the Japanese interviewees intergroup conflict resolution was related to the perception of the past in a way that allowed them to separate the person from the government, which was deemed as responsible. Japanese participants perceived an apology at the intergroup level to be irrelevant for facing and dealing with conflicts. Therefore, it seems understandable that what is expected from Japan internationally and from formerly victimized East Asian nations, cannot or is difficult to be fulfilled by Japan because Japanese mindsets around forgiveness and apologies seems to be qualitatively different compared to those of German participants. Apologies in the East 
Asian context probably do not matter that much, since symbolic acts (such as the exchange of gifts) are more appreciated (Hall, 1976; Tavuchis, 1991).

In the interviews, representations of the past were articulated when the participants talked about forgiveness at the group level. One key term was discovered and coined as historical closure that was already defined previously. In general, closure seemed to be a crucial component of the forgiveness process which is more easily achieved at the individual level than at the group level, since at the individual level there are mostly only two persons involved whereas at the group level many other factors hinder the development of closure.

This is in line with Staub's and Bar-Tal's (2003) reasoning about a psychological departure from the past, which Staub and Bar-Tal (2003) refer to as a symbol of forgiveness. A sense of historical closure can probably develop if there is no competition and controversies over the interpretation of the past, meaning that there is shared understanding of the roots and consequences of the conflict (Staub \& Bar-Tal, 2003; Staub, 2005). Controversies over representations of historical issues can be used by political leaders to widen division between groups. Consequently, based on Study 2 I constructed a scale to capture historical closure. To address a gap in the literature (Exline, Worthington, Hill \& McCullough, 2003), I also explored perceived costs of granting forgiveness in the victim study (Study 3) and potential benefits of seeking forgiveness in the perpetrator study (Study 4).

Theoretically, historical closure should be an associate of forgiveness and an antagonist for the willingness to make amends. Historical closure was conceptualized as an attitude towards the past. Based on theoretical and practical reasoning (Bar-Tal \& Teichman, 2005; Staub \& Bar-Tal, 2003) about the psychological departure from the 
past and its relevance for new peaceful relations, having closure with the past is important for the propensity to forgive, whereas no closure with past should be important for the motivation to make amends.

While in the European context all remaining country variance was explained by historical closure and the costs of forgiveness when these variables were entered into the equation last, in the East Asian context significant variance between the countries remained unexplained in the last step. It could be argued that the differences still found between the countries are a cultural "residual", since the interviews in Study 2 pointed to differences in the conceptualization of forgiveness.

Historical closure was a strong contributor in predicting intergroup forgiveness. Furthermore, historical closure was a strong predictor of the cognitive component to the willingness to make amendments in Germany and Japan. In Germany the degree to which participants were globally conscious contributed to explain variance in the cognitive component of the willingness to make amends, besides the contribution of historical closure. Historical closure seems important for even third generations victims, who have never been directly involved in what has happened during WW II, to move on and allow forgiveness to be an option. For the perpetrator part, lack of historical closure is potentially driving the motivation towards support of former victims on a cognitive level. However, the causality of this assumed underlying mechanism of historical closure has yet to be proven.

In sum, historical closure showed a consistent pattern across the societies and across subject-positions. This indicates that closure with the past is an important concept. From a theoretical point of view, historical closure comes close to healthy commemorations and collective memories (Minow, 1998), since one can argue that 
historical closure may indicate a balance between just enough memories that are not painful anymore, just enough guilt feelings and guilt assignments to be motivated to never let history repeat itself.

\section{What happened to national identification?}

A surprising finding in the German sample was that there was no relationship between national identification and collective guilt and shame. This contradicts previous findings in German samples (Dresler-Hawke \& Liu, 2006) and in general (e.g. Hewstone et al., 2004; McLernon et al., 2004). National identification did not contribute to explain variations as expected. Neither did national identification explain variance in intergroup forgiveness nor was it a mediator between historical closure and forgiveness, as posited in SIT/SCT (Tajfel \& Turner, 1979; Turner, 1999) and previous research on intergroup forgiveness advocating a social identity approach (e.g. Hewstone et al., 2004).

Considering that identity did not contribute as much as expected, it can be assumed that intergroup forgiveness between societies that are geographical distant, in which subject positions are clear, is not only an issue of identification processes. There seems much more behind intergroup forgiveness processes than identity evident in a consistent pattern of historical closure. However, guilt, anger and trust were also important for intergroup forgiveness, but those concepts were not linked to identity. The problem with SIT/SCT is that it does not allow drawing conclusions what the identification with a social category actually entails in terms of their content. It remains an unanswered question what it means to be German or Japanese.

Another surprising result was the positive relationship between national identification and collective guilt in the Japanese sample. However, as already pointed 
out in the discussion in Study 4, there could be several reasons for this positive association. I only can speculate about them, but the positive associations contradict theoretical propositions about the role of national identification in social identity theory, since it is opposed to the motivation to maintain a positive social identity by keeping distance from the dark parts of what one's group has committed (Tajfel \& Turner, 1979; Turner et al., 1987; Turner, 1999).

However, it is not necessarily a contradiction in an East Asian or specifically in the Japanese context, since Gries and Peng (2002) argued that Eastern reasoning could stand contradictions. Therefore, the positive association that seemed contradictory from a Western logic point of view can actually be meaningful in the Japanese context. Furthermore, although previous studies suggest that younger generations in Japan are apolitical (Liu \& Atsumi, 2008), the internet among other means of transmission of knowledge may have a crucial impact on young Japanese's perceptions of their past (Morris-Suzuki \& Rimmer, 2002).

Another explanation comes from political scientists. Historically, Japanese have shifted in their opinions about other countries between an inferiority and superiority complex (Li, Sabella, \& Liu, 2002; Reischauer, 1988). Having been defeated by the US and rebuilt under their influence after WW II, many Japanese today tend to maintain an inferiority complex vis-à-vis the US, since Japan had to rely on the US for security and economic recovery after WW II (Donahue, 1998).

In contrast, Japanese tend to have a superiority complex towards East Asian neighbours. This is because of Japan's rise as an economic superpower during the last decades, making them feeling more advanced and superior than their East Asian neighbours. If Japanese perceive East Asian neighbours as inferior, it can be argued that 
those are Japanese who identify highly as Japanese. Furthermore, it can be speculated that Japanese who feel superior towards East Asian neighbours can easily apologise or support amends on a cognitive level. They probably can afford to do so, since they are cognitively on a higher ground. Perhaps they feel secure about their identity and satisfied by giving a noble, symbolic gesture. Unfortunately, SIT and SCT do not allow taking into account how secure Japanese may feel about being Japanese or how secure one is feeling about identifying with their nation in general.

In public, the Japanese government is reluctant to apologise "appropriately" which is probably based on a strategy to win votes from more conservative Japanese. However, the results suggest that there may be difference between the government and how everyday Japanese feel about these issues.

\section{"Shame” versus "Guilt” Cultures}

Anthropological theorists (e.g. Benedict, 1946) have argued that Japan is a "shame" culture. Although this view has been contested (e.g. Creighton, 1990) it has been applied in psychological research on intergroup conflict to distinguish between Japan as a "shame" culture and Germany as a "guilt" culture (see Páez et al., 2006). Based on this distinction, I expected that Japanese participants would experience more shame than guilt feelings. The results showed a different pattern, since Japanese participants reported more guilt feelings than shame feelings and both collective emotions were higher than in the German sample.

It is argued that collective guilt feelings are experienced when an incident seems under the control of the ingroup (Branscombe \& Doosje, 2004), whereas collective shame feelings are experienced due to certain degrading characteristics of the ingroup (Brown et al., 2008). Japan and China are in constant tensions over controversies 
regarding the "correct" historical representations of past harm done by Japan during WW II and the Sino-Japanese War (Reilly, 2006). There is also an international debate about the Nanjing Massacre and the Comfort Women, with eyewitnesses delivering emotional testimonies. It seems reasonable to argue that this whole debate has an impact on young Japanese participants, since they have access to these debates via multiple media outlets (Morris-Suzuki \& Rimmer, 2002).

Japanese university students in particular could feel bad on behalf of their country, due to the fact that their government is still downplaying past harm done and causing grievances and resentment in other East Asian countries, which is something that can be prevented and may be controllable. This may have led to the high experience of collective guilt by Japanese participants. In the German sample, the experience of collective guilt and shame were relatively low compared to the Japanese participants. This can be interpreted in light of the issues being more resolved in the European post WW II context than in Japan, which supports claims in the literature (Oliner, 2008).

Therefore, labelling a culture as inherently shame-prone or guilt-prone may be a too simplistic approach that does not take the historical context and interpretations of the conflict into consideration. As this study has demonstrated, the historical context is a rich resource for explanations of the differences between Japanese participants and German participants and the European and East Asian context respectively.

\section{Intergroup forgetting versus intergroup forgiveness}

In Study 2, there were some hints pointing to different cultural conceptualizations of forgiveness. The Japanese interviewees did not seem to value forgiveness as a response to harm done. This can be a coincidence. On the other hand, the alternative preferences over forgiveness could be potentially explained by culture- 
specific factors. The Japanese participants preferred either to downplay the significance of the harm done in forgetting about it ("Cause I forget ... I don't need to forgive" [J1]), withdrawal or avoidance, and emphasizing the importance of maintaining superficial harmony in line with theoretical propositions specific to Asian cultures (Brew, 2007; Huang, Jone, \& Peng, 2007). Forgiveness was considered, but only when it was useful to benefit the relationships. This is in support of Markus and Kitayama's (1991) distinction between independent and interdependent selves. The latter is more important to Asian cultures in which the interconnectedness of the self with others is more relevant.

Moreover, attaching shame to conflict situations is another culture specific factor that deserves attention in the overall process. The notion of feeling ashamed of being perceived as not being able to deal with social situations effectively to promote peaceful relationships and avoid conflict is tightly related to the Asian concept of face saving. This may explain why Japanese participants preferred avoidance and forgetting over actually dealing with the conflict, since the purpose of this response might be to avoid face-threatening situations (Ting-Toomey, 2005). First, the Japanese participants saw only an instrumental value of forgiveness (e.g. a more committed relationship) and not as a means to release oneself from the burden of a conflict. Second, a means of controlling emotions to save face was articulated as either ending the relationship or forgetting about the incident.

When something is forgotten, it does not necessarily imply that the harm done and all ill feelings associated with it vanished (e.g. Enright \& North, 1999). Furthermore, forgiveness does not "happen" automatically as forgetting does in the first place (Margalit, 2005). Forgetting is probably a mere impulse, when something terrible 
happen, since the first thing that probably happens is not to think about it which is not a decision, but happens automatically. Furthermore, it could be argued that, if forgetting prevails it seems logical impossible to forgive, since the harm done is not any longer cognitively available that can be forgiven. Margalit (2005) argued that forgiveness is more based on disregarding the painful acts rather than forgetting them. Since historical closure was strongly associated with intergroup forgiveness, it could be speculated that it may be different from forgetting and similar to disregarding the harm committed. This is only cautiously suggested, since there is no data to prove it.

The problem with suppressing memories of the harm done is that they can break out as soon as people are reminded (e.g. in the media). Therefore, forgetting will probably not help to overcome resentment - which is arguably the focus of forgiveness - since the necessary processes have not been undergone. In contrast, forgiveness is a conscious matter. It will probably not happen automatically, since the perpetrator side has to show some kind of remorse, although the past cannot be changed by doing this. Based on social representations of history it could be argued that acknowledgment of past harm done by a higher level representative may help to re-interpret the past and may generate new (non-conflicting) social representations of the past. The form of this acknowledgment may be different across cultures as I elaborated earlier. Generally speaking, forgetting may have nothing to do with forgiving, but both are linked to each other in everyday language ("forgive and forget").

\section{Revisiting the process of intergroup forgiveness}

To recount, I initially proposed a working definition of intergroup forgiveness in Chapter 2 by contrasting interpersonal from intergroup forgiveness. Based on the 
findings in this thesis, intergroup forgiveness as a process should be described as follows.

Intergroup forgiveness is a contextualized, dynamic process between the victimized and perpetrating party that involves negotiation and understanding. Historical closure is an integral part of the intergroup forgiveness process. Closure with the past is not related to forgetting, but involves strategically moving past ill feelings attached to the past atrocity or conflict while disregarding the reasons for the act of violence and the act as such. Closure with the past symbolizes social healing that can eventuate in reconciliation. It may be an unfolding and continuous process. The perpetrator party has to give reasons for the victimized party to consider forgiveness as a possible response. The victimized party then has to reconsider their grievances. The process entails acknowledgment of harm done and a public apology that includes significant and coherent actions, and an acceptance by the victimized party that the approach by the perpetrator party is appropriate and genuine, leading to an open dialogue. Through the process of the acknowledgment of and remorse for the harm done, which should entail symbolic acts, a re-interpretation of the past is possible, that can generate more consensual social representations of the past. This shared and consensual interpretation of the conflict is necessary in order to be able to empathize and gain trust with the other side.

\section{Limitations and suggestions for future research}

This thesis contributes to a better understanding of intergroup forgiveness. However, conclusions drawn from the results deserve further validation and calls for future research. Unfortunately, only a limited number of countries were available to conduct the cross-national meta-analysis, which was based on one instrument that was 
widely used. The meta-analysis would have been much more powerful if more countries would have been included. However, the context effects revealed are impressive and should not be ignored. Future research could broaden the focus, apply a domain-based approach and gather studies that assess the construct forgiveness at the individual and at the group level in order to evaluate at what level which contextual variables contribute to explain variance.

Since forgiveness at the group level is still poorly understood, qualitative approaches can reveal different factors, such as cultural factors, contributing to our understanding of this process. Study 2 could have benefited from expanding the number of interviews and also from conducting focus groups in order to be able to draw more precise conclusions. The inclusion of participants from a European victim nation would have contributed to a more balanced sampling. Therefore, future research could focus on a series of interviews that allows for a deeper examination of culture-specific factors.

Study 2 revealed hints at cross-cultural differences and culture-specific factors possibly influencing the conceptualisation of forgiveness. It is important to establish an understanding of how (and whether) forgiveness works in a specific cultural context. Western forms of forgiveness seem to be anchored in Christianity and the example of how Christ responded to people who persecuted him. It is crucial to establish how and whether the concept of forgiveness is central to peace and reconciliation in nonChristian societies in future research. The Asian concept of face, for instance, may mitigate against some of the more emotional processes of apology and acceptance of apology typical in Western/Christian cycles of forgiveness.

The survey studies provided initial evidence that the perception and interpretation of the historical past has an impact on forgiveness and willingness to 
make amends respectively. The survey studies were conducted with university students. It would be interesting to conduct similar studies with a general population sample in order to reveal whether the effects still hold with a non-university population.

The instruments used should be refined in future research. Since the relationships with identity were weak, alternative instruments to assess collective identity should be employed to rule out the possibility that the lack of relationship was due to the measurement of identity. Future studies could benefit from using additional instrument to assess social representations of history, which was not the case in the current thesis. It would also be interesting to focus on different levels of inclusiveness of identification (e.g. superordinate identity). Future studies could benefit from using additional instrument to assess social representations of history, which was not the case in the current thesis. Historical closure was initially tested - although it was not the focus of the study - for convergent validity, but not for discriminant validity. Therefore, a thorough internal and external validation and refinement of the concept historical closure deserves some further attention. The instrument used was based on six items for Study 3 and four items for Study 4. Future research could address the functions and different levels of historical closure as well as elaborating on how it is distinct from forgetting.

However, all conclusions drawn must be regarded with caution, since these were cross-sectional studies not allowing inference of causality. Therefore, future research could focus on longitudinal studies or experimental designs, such as scenario-based studies, in order to be able to draw causal conclusions. Finally, the literature on intergroup forgiveness can benefit from applying what has been learnt in different conflict contexts across different cultural contexts. 


\section{Concluding remarks}

The current thesis has supported theoretical propositions of social representations of history (Hilton \& Liu, 2008; Liu \& Hilton., 2005) and the significance of the historical context for intergroup forgiveness. The emergence of consistent patterns of the new concept historical closure seemed to be a key element in the intergroup forgiveness process across countries and across subject positions. The findings have implications for the practical applications of intergroup forgiveness. Staub and Bar-Tal (2003) and Staub (2005) advocated an approach to achieve a shared understanding of the roots of a conflict in order to foster social healing which includes forgiveness and eventuates in reconciliation. The current thesis provided empirical evidence that the intergroup forgiveness process is indeed very different from interpersonal forgiveness. The description of the process entails that coherent acts by the perpetrator party may allow developing new social representations of the past that are shared between victim and perpetrator parties.

One way to implement these findings to foster forgiveness and the reconciliation of relationships is through education. Textbook commissions after WW II in Europe have shown that it seems possible to create a shared account of the past with which formerly conflicting parties can agree on. This thesis indicated that education and school curricula might have influence and enable the reduction of negative enemy stereotypes, which was the aim of the textbook commissions. A shared understanding of the origin of the conflict or in this case the war and its consequences may foster historical closure - at least for the victim part - and lead towards forgiveness. This research suggested that a Japanese sample of university students understood their government caused tensions between Japan and her East Asian neighbours. 
Furthermore, they may not agree on what the current Japanese government is claiming. It is a question for future policy makers how to mediate this conflict between government and citizens. 


\section{References}

(* articles included in the meta-analysis in Study 1)

*Allan, A., Allan, M. M., Kaminer, D., \& Stein, D. J. (2006). Exploration of the association between apology and forgiveness amongst victims of human rights violations. Behavioral Sciences \& the Law. Special Issue: Gender and Psychopathy, 24, 87 - 102.

*Allemand, M., Amberg, I., Zimprich, D., \& Fincham, F. D. (2007). The role of trait forgiveness and relationship satisfaction in episodic forgiveness. Journal of Social \& Clinical Psychology, 26, 199 - 217. *

Allport, G. W. (1954). The nature of prejudice. Cambridge: Perseus Books.

Amstutz, M. R. (2005). The healing of nations: The promise and limits of political forgiveness. Lanham, MD: Rowan \& Littlefield Publishers, Inc.

Andrew, M. (2000). Forgiveness in Context. Journal of Moral Education, 29, 75 - 86.

Atsumi, T., Suwa, K., Kobayahi, H., Miyamoto, T., \& Seki, Y. (in press). Toward reconciliation of historical conflict between Japan and China: Design science for peace in Asia. In C. Montiel \& N. Noor (eds.) Peace Psychology in Asia. Springer.

Azar, F., \& Mullet, E. (2002). Muslim and Christian in Lebanon: Common Views Regarding Political Issues. Journal of Peace Research, 39(6), 755 - 766.

Azar, F., Mullet, E., \& Vinsonneau, G. (1999). The propensity to forgive: Findings from Lebanon. Journal of Peace Research, 36, 169 - 181.

Bar-Tal, D. (2000). Shared Beliefs in a Society: Social Psychological Analysis. Thousand Oaks: Sage Publications.

Bar-Tal, D., \& Teichman, Y. (2005). Stereotypes and prejudice in conflict: representation of Arabs in Israeli Jewish society. New York: Cambridge University Press.

Basabe, N. \& Valencia, J. (2007). Social structure, culture of peace and emotional climate. Journal of Social Issues, 63, 405 - 419.

Bassiri, D. (1988). Large and small sample properties of maximum likelihood estimates for the hierarchical linear model. Doctoral Dissertation Michigan State University, USA. 
Baumeister, R. F., Exline, J. J., \& Sommer, K. L. (1998). The victim role, grudge theory, and two dimensions of forgiveness. In E.L. Worthington, Jr. (Ed.), Dimensions of forgiveness: Psychological research and theological principles (pp. 79 - 106). Philadelphia: Templeton Foundation Press.

Beatty, J. (1970). Forgiveness. American Philosophical Quarterly, 7, 246 - 252.

Benedict, R. (1946). The Chrysanthemum and the Sword: Patterns of Japanese Culture. Boston: Houghton Miller.

Berry, J.W. (1976). Human ecology and cognitive style: Comparative studies in cultural and psychological adaptation. New York: Sage/Halsted/Wiley.

Bierbrauer, G. (1992). Reactions to Violation of Normative Standards: A Cross-cultural Analysis of Shame and Guilt. International Journal of Psychology, 27, 181 193.

Bindenagel, J. D. (2002). Entschädigung und Wiedergutmachung, Europäische Rundschau, 30, 93 - 106.

Borg, I., \& Groenen, P. (1997). Modern multidimensional scaling. Theory and applications. New York: Springer-Verlag.

Branscombe, N. R., \& Doosje, B. (2004). Collective Guilt: International Perspectives. Cambridge, UK: Cambridge University Press.

Braun, V., \& Clarke, V. (2006). Using thematic analysis in psychology. Qualitative Research in Psychology, 3, 77 - 101.

Breakwell, G. M. (2001). Social representational constraints upon identity. In K. D. Deaux \& G. Philogène (Eds.). Representations of the social (pp. 271 - 284). Malden: Blackwell Publishing.

Brehm, J., \& Rahn, W. (1997). Individual-level evidence for the causes and consequences of social capital. American Journal of Political Science, 41, 999 1023.

Brew, F. (2007). Harmony and Controversy: the Yin and Yang of Conflict in East Asian and Western Cultures. In J.H. Liu, C. Ward, A. Bernardo, M. Karasawa, \& R. Fischer (eds.) Progress in Asian Social Psychology Volume 6. Seoul: Kyoyook Kwahasaka.

Brewer, M. B. (1979). Ingroup bias in the minimal intergroup situation: A cognitive motivational analysis. Psychological Bulletin, 86, 475 - 482. 
Brewer, M. B. (1999). The nature of prejudice: Ingroup love or outgroup hate? Journal of Social Issues, 55, 429 - 444.

Brewer, M. B. (2001). Social identities and Social representations: a question of priority? In K. D. Deaux \& G. Philogène (Eds.). Representations of the social (pp. 305-311). Malden: Blackwell Publishing.

Brewer, M. B., Manzi, J. M., \& Shaw, J. S. (1993). In-group identification as a function of depersonalization, distinctiveness, and status. Psychological Science, 4, 88 92.

Brown, R., \& Čehajić, S. (2008). Dealing with the past and facing the future: mediators of the effects of collective guilt and shame in Bosnia and Herzegovina. European Journal of Social Psychology, 38, 669 - 684.

Bruner, J. C. (1990). Acts of Meaning. Cambridge, Mass.: Harvard University Press.

Buruma, I. (1994). The Wages of Guilt: memories of war in Germany and Japan. NY: Farrar, Straus and Giroux.

Cairns, E., \& Roe, M. D. (Eds.). (2003). The role of memory in ethnic conflict. New York: Palgrave Macmillian.

Cairns, E., Hewstone, M., Niens, U., \& Tam, T. (2005). Intergroup forgiveness and intergroup conflict: Northern Ireland, a case study. In E. L. Worthington (Ed.), Handbook of forgiveness (pp. 461 - 476). New York: Brunner-Routledge.

*Carson, J. W., Keefe, F. J., Goli, V., Fras, A. M., Lynch, T. R., Thorp, S. R., et al. (2005). Forgiveness and chronic low back pain: A preliminary study examining the relationship of forgiveness to pain, anger, and psychological distress. The Journal of Pain, 6, 84 - 91.

Čehajić, S., Brown, R., \& Castano, E. (2008). Forgive and Forget? Antecedents and Consequences of Intergroup Forgiveness in Bosnia and Herzegovina. Political Psychology, 29, 351 - 367.

Chai, A. Y. (1993). Asian-Pacific Feminist Coalition Politics: The Chongshindae/Jugunianfu (Comfort Women) Movement. Korean Studies, 17, 67 $-91$.

Chan, C. P., \& Bridges, B. (2006). China, Japan, and the clash of Nationalism. Asian perspectives, 30, 127 - 156.

Chang, I. (1998). The rape of Nanking: the forgotten holocaust of World War II. New 
York, NY: BasicBooks.

Chapman, A. R. (2007). Truth Commissions and Intergroup Forgiveness: The Case of the South African Truth and Reconciliation Commission. Peace \& Conflict, 13, 51.

Coday, D. J. (1998). 'I cannot die without an apology': Korean women forced into sexual slavery await words that will ease torment. National Catholic Reporter, $35,14-15$.

Cody, E. (2005, April 20). In China, Roots Of Anger Toward Japan Run Deep; Lasting Sting of Wartime Atrocities Fuels Protests Over Moves by Tokyo. The Washington Post, p. A12.

Cohen, A. (2006, May 22). Apologize. Fully. Finally. The Globe and Mail, p. A13.

Cohen, J., Cohen, P., West, S. G., \& Aiken, L. S. (2003). Applied multiple regression/correlation analysis for the behavioural sciences. Hillsdale, NJ: Lawrence Erlbaum.

Conrad, S. (2003). Entangled Memories: Versions of the Past in Germany and Japan, 1945-2001. Journal of Contemporary History, 38, 85 - 99.

Coyle, C. T., \& Enright, R. D. (1997). Forgiveness in Intervention with postabortion men. Journal of Consulting and Clinical Psychology, 65, 1042 - 1046.

Creighton, M. R. (1990). Revisiting shame and guilt cultures: A forty-year pilgrimage, Ethos, 18, 279 - 307.

Dekker, S. \& Fischer, R. (2008). Cultural Differences in Academic Motivation Goals: A meta-analysis across thirteen societies. Journal of Educational Research, 101, 99 $-110$.

Denton, R. T. \& Martin, M. W. (1998). Defining forgiveness: An empirical exploration of process and role. The American Journal of Family Therapy, 26, 281 - 292.

Diener, E., Diener, M., \& Diener, C. (1995). Factors predicting the subjective wellbeing of nations. Journal of Personality and Social Psychology, 69, 851 - 864.

Digeser, P. E. (2001). Political Forgiveness. Ithaca and London: Cornell University Press.

Dixon, J., Durrheim, K., \& Tredoux, C. (2005). Beyond the Optimal Contact Strategy: A Reality Check for the Contact Hypothesis. American Psychologist, 60, 697 711. 
Dodds, G. (2003). Political apologies: Chronological List. Retrieved April 5, 2009, from http://reserve.mg2.org/apologies.htm

Donahue, R. T. (1998). Japanese Culture and Communication: Critical Cultural Analysis. University Press of America.

Doosje, B., Branscombe, N. R., Spears, R., \& Manstead, A. S. R. (1998). Guilty by association: When one's group has a negative history. Journal of Personality and Social Psychology, 75, 872 - 886.

Dovidio, J. F., Gaertner, S. L., \& Kawakami, K. (2003). Intergroup Contact: The Past, Present, and the Future. Group Processes \& Intergroup Relations, 6, 5 - 21.

Dresler-Hawke, E., \& Liu, J. H. (2006). Collective shame and the positioning of German national identity, Psicologia Politica, 32, 131 - 153.

Duveen, G. (2001). Representation, Identities, Resistance. In K. D. Deaux \& G. Philogène (Eds.). Representations of the social (pp. 271 - 284). Malden: Blackwell Publishing.

Enright, R. D. (2001). Forgiveness is a choice. Washington DC: APA Life Tools.

Enright, R. D., \& North, J. (Eds.). (1998). Exploring Forgiveness. London: The University of Wisconsin Press.

Enright, R. D., \& Rique, J. (2000). The Enright Forgiveness Inventory. Mind Garden.

Enright, R. D., \& the Human Development Study Group. (1991). The Moral Development of Forgiveness. In W. M. Kurtines \& J. L. Gerwitz (Eds.), Handbook of Moral Behavior and Development (Vol. 1: Theory, pp. 123 - 148). Hillsdale, New Jersey: Lawrence Erlbaum Associates, Inc.

Enright, R. D., Freedman, S., \& Rique, J. (1998). The psychology of interpersonal forgiveness. In R. D. Enright \& J. North (Eds.), Exploring forgiveness (pp. 46 62). Madison: University of Wisconsin Press.

Enright, R. D., Gassin, E. A., \& Wu, C. (1992). Forgiveness: a development view. Journal of Moral Education, 21, 99 - 114.

Enright, R. D., Santos, M. J., \& Al-Mabuk, R. (1989). The adolescent as forgiver. Journal of Adolescence, 12, 99 - 110.

Ergüner-Tekinalp, B. (2007). Forgiveness of historical and current racial offenses: A study of intergroup forgiveness among African Americans. Unpublished Ph.D., Auburn University, United States - Alabama. 
Exline, J. J., Worthington, E. L., Jr., Hill, P., \& McCullough, M. E. (2003). Forgiveness and Justice: A Research Agenda for Social and Personality Psychology. Personality and Social Psychology Review, 7, 337 - 348.

Feldman, L. G. (1999). The principle and practice of 'reconciliation' in German foreign policy: relations with France, Israel, Poland and the Czech Republic. International Affairs, 75, 333 - 356.

Fincham, F. D., Jackson, H., \& Beach, S. R. H. (2005). Transgression severity and forgiveness: Different moderators for objective and subjective severity. Journal of Social \& Clinical Psychology, 24, 860 - 875.

Fischer, R. \& Chalmers, A. (2008). Is optimism universal? A meta-analytical investigation of optimism levels across 22 nations. Personality and Individual Differences, 45, 378 - 382.

Fischer, R. \& Hanke, K. (2009). Are societal values linked to global peace and conflict? Peace \& Conflict: Journal of Peace Psychology, 15, 227 - 248.

Fischer, R. (2008). Rewarding seniority: investigating organizational and cultural determinants of seniority-based allocations. Journal of Social Psychology, 148, $167-186$.

Fischer, R., \& Mansell, A. (2007). Levels of organizational commitment across cultures: A meta analysis. Paper in review. Journal of International Business Studies.

Freedom House. (no date). Annual survey of freedom country scores 1972-1973 to 1998-1999. Washington, D.C. Publication. Retrieved September 27, 1999: http://www.freedomhouse.org/rankings.pdf

Frei, N. (2009). NS-VERFAHREN - „Die Deutschen sind sich selbst schuldig, gegen Demjanjuk vorzugehen“. Retrieved May 25, 2009 from http://www.spiegel.de/politik/deutschland/0,1518,626409,00.html

Fukuyama, F. (1992). The end of history and the last man. London: Penguin.

Gaertner, S. L., \& Dovidio, J. F. (2000). Reducing intergroup bias: The common ingroup identity model. New York: Psychology Press.

Georgas, J., van de Vijver, F. J. R. \& Berry, J. W. (2004).The Ecocultural Framework, Ecosocial Indices, and Psychological Variables in Cross-Cultural Research. Journal of Cross-Cultural Psychology, 35, 74 - 79.

Glass, G. V. (1976). Primary, secondary and meta-analysis research. Educational 
researcher, $5,3-8$.

Glynos, J., \& Howarth, D. (2007). Retroduction. In J. Glynos \& D. Howarth, Logics of Critical Explanation in Social and Political Theory (pp. 18 - 48). Abingdon: Routledge.

Govier, T. (2002). Forgiveness and Revenge. London and New York: Routledge.

Grbich, C. (2007). Qualitative data analysis: an introduction. London: Sage.

Greenfield, P. M. (2000). Three approaches to the psychology of culture: Where do they come from? Where can they go? Asian Journal of Social Psychology, 3, 223 240.

Gries, P., \& Peng, K. (2002). Culture Clash? Apologies East and West. Journal of Contemporary China, 11, 173 - 178.

Hall, E. T. (1976). Beyond culture. Garden City, N.Y.: Anchor Press.

Hamber, B. (2007). Forgiveness and reconciliation: Paradise lost or pragmatism? Peace and Conflict: Journal of Peace Psychology, 13, 115 - 125.

Hamber, B., \& Wilson, R. (2003) Symbolic closure through memory, reparation and revenge in post-conflict societies. In E. Cairns, \& M. D. Roe (Eds.). The role of memory in ethnic conflict. (pp. 144 - 165). New York: Palgrave Macmillian.

Hanke, K., Liu, J. H., \& Fischer, R. (2008). Perspectives on forgiveness across different cultures: Distinctions between interpersonal and intergroup forgiveness. Paper presented at the Annual meeting of the Society of Australasian Social Psychologists (SASP), Wellington, New Zealand.

Hanke, K., Liu, J. H., Fischer, R., \& Vauclair, C. M. (2007). The value of forgiveness in a cultural context: Conceptualizing intergroup and individual-level forgiveness. Unpublished manuscript.

Hargrave, T. D., \& Sells, J. N. (1997). The development of a forgiveness scale. Journal of Marital and Family Therapy, 23, 41 - 64.

Hartwell, M. (2005). Perceptions of Justice, Identity and Political Processes of Forgiveness and Revenge in Early Post-Conflict Transitions, Case Studies: Northern Ireland, Serbia, South Africa. Ph.D thesis, Oxford University.

Hauss, C. (2003). Apology and Forgiveness. In G. Burgess \& H. Burgess (Eds.) Beyond Intractability, $\quad$ Retrieved Jan 25, 2009 from <http://www2.beyondintractability.org/m/apology_forgiveness.jsp > Conflict 
Research Consortium, University of Colorado, Boulder.

Hedges, L. V. \& Olkin, I. O. (1985). Statistical Methods for Meta Analysis. Orlando Florida, Academic Press.

Hein, L. E., \& Selden, M. (2000). Censoring history citizenship and memory in Japan, Germany, and the United States. New York: M.E: Sharpe.

Hewstone, M., Cairns, E., Voci, A., Hamberger, J., \& Niens, U. (2006). Intergroup Contact, Forgiveness, and Experience of "The Troubles" in Northern Ireland. The Journal of Social Issues, 62, 99 - 120.

Hewstone, M., Cairns, E., Voci, A., McLernon, F., Niens, U., \& Noor, M. (2004). Intergroup Forgiveness and Guilt in Northern Ireland: Social Psychological Dimensions of 'The Troubles'. In N. R. Branscombe \& B. Doosje (Eds.), Collective Guilt: International Perspectives (pp. 193 - 215). Cambridge: University Press.

Hicks, G. (1999). The Comfort Women Redress Movement. In R. L. Brooks (Ed.), When Sorry isn't Enough: The Controversy Over Apologies and Reparations for Human Injustice (pp. 113 - 125). New York: New York University Press.

Hilton, D. J., \& Liu, J. H. (2008). Culture and inter-group relations. The role of social representations of history. In R. Sorrentino \& S. Yamaguchi (Eds.) The Handbook of Motivation and Cognition: The Cultural Context, (pp. 343 - 368). New York: Guilford.

Hogg, M. A., \& Williams, K. D. (2000). From I to we: Social identity and the collective self. Group Dynamics: Theory, Research, and Practice, 4, 81 - 97.

Hong, Y.-Y., \& Chiu, C.-Y. (2001). Toward a paradigm shift: from cross-cultural differences in social-cognition to social-cognitive mediation of cultural differences, Social Cognition, 19, 181 - 196.

Hong, Y.-Y., Morris, M. W., Chiu, C.-Y., \& Benet-Martínez, V. (2000). Multicultural Minds - a dynamic constructivist approach to culture and cognition, American Psychologist, 55, 709 - 720.

Hornsey, M. J. (2008). Social Identity Theory and Self-categorization Theory: A Historical Review. Social and Personality Psychology Compass, 2, 204 - 222.

Huang, L. L., Jone, K. Y., \& Peng, T. K. (2007). Conflict Resolution Patterns and Relational Context: An Exploratory Study Combining Etic and Emic Theories in 
Taiwan. In J. H. Liu, C. Ward, A. Bernardo, M. Karasawa \& R. Fischer (Eds.), Progress in Asian Social Psychology Volume 6. Seoul: Kyoyook Kwahasaka.

*Hui, E. K. P., \& Ho, D. K. Y. (2004). Forgiveness in the context of developmental guidance: Implementation and evaluation. British Journal of Guidance \& Counselling, 32, 471 - 492.

Humana, C. (1986). World human rights guide. London: The Economist Publications.

Hunter, J. E., \& Schmidt, F. L. (2004). Methods of meta-analysis: Correcting error and bias in research findings ( $2^{\text {nd }}$ ed.). Thousand Oaks: Sage Publications, Inc.

Ignatieff, M. (1996). There's no place like home: The politics of belonging. In S. Dunant \& R. Porter (Eds.) The Age of Anxiety London: Virago Press.

Inglehart, R. \& Baker, W. E. (2000). Modernization, culture change, and the persistence of traditional values. American Sociological Review, 65, 19-51.

Inglehart, R. (1997). Modernization and post-modernization. Cultural, economic and political change in 43 countries. Princeton, NJ: Princeton University Press.

Iyer, A., Schmader, T., \& Lickel, B. (2007). Why individuals protest the perceived transgressions of their country: The role of anger, shame and guilt. Personality and Social Psychology Bulletin, 33, 572 - 587.

James, L. J., \& Williams, L. (2000). The cross-level operator in regression, ANCOVA, and contextual analysis. In K. J. Klein \& S. W. J. Kozlowski (Eds.), Multilevel Theory, Research, and Methods in Organizations (pp. 382 - 424). San Francisco: Jossey Bass.

Janover, M. (2005). The Limits of Forgiveness and the Ends of Politics, Journal of Intercultural Studies, 26, 221 - 235.

Jose, P. E. (2003) MedGraph-I: A programme to graphically depict mediation among three variables: The internet version, version 2.0. Victoria University of Wellington, Wellington, New Zealand. Retrieved [date] from http://www.victoria.ac.nz/staff/paul-jose-files/medgraph/medgraph.php.

Kadima Kadiangandu, J. \& Mullet, E. (2007). Intergroup forgiveness: A Congolese perspective. Peace and Conflict: Journal of Peace Psychology, 13, 35 - 47.

Kadima Kadiangandu, J., Gauché, M., Vinsonneau, G., \& Mullet, E. (2007). Conceptualizations of forgiveness: Collectivist-Congolese versus IndividualistFrench viewpoints. Journal of Cross-Cultural Psychology, 38, 432 - 437. 
Kamat, V. I., Jones, W. H., \& Lawler-Row, K. A. (2006). Measuring forgiveness as a dimension of personality. Individual Differences Research, 4, 322 - 330.

Kanz, J. E. (2000). How do people conceptualize and use forgiveness? The Forgiveness Attitudes Questionnaire. Counselling \& Values, 44, 174 - 186.

Karremans, J. C., van Lange, P. A. M., Ouwerkerk, J. W., \& Kluwer, E. S. (2003). When forgiving enhances psychological well-being: The role of interpersonal commitment. Journal of personality and social psychology, 84, 1011 - 1026.

Kearns, J. N. \& Fincham, F. D. (2004). A Prototype Analysis of Forgiveness. Personality and Social Psychology Bulletin, 30, 838 - 855.

Kim, R. (2001, September 7). Survivors protest anniversary / Testimony about Rape of Nanking. San Francisco Chronicle, p. A14.

Klein, K. J., Bliese, P. D., Kozlowski, S. W. J., Dansereau, F., Gavin, M. B., Griffin, M. A., Hofmann, D. A., James, L. R., Yammarino, F. J.,\& Bligh, M. C. (2000). Multilevel analytical techniques: Commonalities, differences, and continuing questions. In K. J. Klein \& S.W.J. Kozlowski (Eds.), Multilevel theory, research and methods in organizations: Foundations, extensions, and new directions (pp. 512 - 553). San Francisco, CA: Jossey-Bass.

Kohlberg, L. (1976). Moral Stages and moralization: the cognitive-developmental approach. In T. Lickona (Ed.), Moral development and behaviour: Theory, research and social issues (pp. 31 - 53). New York: Holt.

Krause, N., \& Ellison, C. G. (2003). Forgiveness by God, forgiveness of others, and psychological well-being in late life. Journal for the Scientific Study of Religion, 42, $252-276$.

László, J. (2003). History, Identity, Narratives. In J. László, \& W. Wagner (Eds.) (2003). Theories and Controversies in Societal Psychology. (pp. 180 - 192) Budapest: New Mandate.

László, J., \& Wagner, W. (Eds.) (2003). Theories and Controversies in Societal Psychology. Budapest: New Mandate.

Lawler, K. A., Younger, J. W., Piferi, R. L, Billington, E., Jobe, R., Edmondson, K., et al. (2003). A change of heart: Cardiovascular correlates of forgiveness in response to interpersonal conflict. Journal of Behavioral Medicine, 26, 373 393. 
Lazare, A. (2004). On apology. Oxford: Oxford University Press.

Leach, W. L., van Zomeren, M., Zebel, S., Vliek, M. L. W., Pennekamp, S. F., Doosje, B., et al. (2008). Group-Level Self-Definition and Self-Investment: A Hierarchical (Multicomponent) Model of In-Group Identification. Journal of Personality and Social Psychology, 95, 144 - 165.

Lewicki, R. J., \& Wiethoff, C. (2000). Trust, trust development, and trust repair. In M. Deutsch \& P. Coleman (Eds.), The handbook of conflict resolution: Theory and practice (pp. 86-107). San Francisco: Jossey-Bass.

Li, F., Sabella, R., \& Liu, D. (2002). Nanking 1937: Memory and Healing. Armonk, N.Y: M.E. Sharpe.

Lickel, B., Miller, N., Stenstrom, D. M., Denson, T. F., \& Schmader, T. (2006). Vicarious Retribution: The Role of Collective Blame in Intergroup Aggression. Personality and Social Psychology Review, 10, 372 - 390.

Lipsey, M. W., \& Wilson, D. B. (2001). Practical Meta-Analysis. Sage: Thousand Oaks, CA.

Liu, J. H, Yamagishi, T., Wang, F. X., Schug, J., Lin, Y. C., Huang, L. L., \& Yu, S. H. (2009). Unbalanced triangle in the social dilemma of trust: Internet studies of realtime real money social exchange between China, Japan, and Taiwan. Under review, Applied Psychology: An International Review.

Liu, J. H., \& Atsumi, T. (2008). Historical conflict and resolution between Japan and China: Developing and applying a narrative theory of history and identity. In T. Sugiman, K. J. Gergen, W. Wagner \& Y. Yamada (Eds.), Meaning in action: Constructions, narratives, and representations (pp. 327 - 344). Tokyo: SpringerVerlag.

Liu, J. H., \& Hilton, D. (2005). How the past weighs on the present: Social representations of history and their role in identity politics. British Journal of Social Psychology, 44, 537 - 556.

Liu, J. H., \& László, J. (2007). A narrative theory of history and identity: Social identity, social representations, society and the individual. In G. Moloney \& I. Walker (Eds.), Social representations and identity: Content, process and power (pp. 85 107). London: Palgrave Macmillan.

Liu, J. H., Wilson, M. W., McClure, J., \& Higgins, T. R. (1999). Social identity and the 
perception of history: Cultural representations of Aotearoa/New Zealand. European Journal of Social Psychology, 29, 1021 - 1047.

Liu, J. H., \& Sibley, C. G. (in press). Culture, social representations, and peacemaking: A symbolic theory of history and identity. In C. Montiel \& N. Noor (eds.) Peace Psychology in Asia. New York: Springer.

Liu, J. H., Goldstein-Hawes, R., Hilton, D. J., Huang, L. L., Gastardo-Conaco, C., Dresler-Hawke, E., Pittolo, F., Hong, Y. Y., Ward, C., Abraham, S., Kashima, Y., Kashima, E., Ohashi, M., Yuki, M., \& Hidaka, Y. (2005). Social representations of events and people in world history across twelve cultures. Journal of Cross-Cultural Psychology, 36, 171 - 191.

Liu, J. H., Páez, D., Hanke, K., Rosa, A., Hilton, D. J., Sibley, C. G., et al. (2009). Cross-cultural dimensions of meaning in the evaluation of events in world history? Perceptions of historical calamities and progress in cross-cultural data from 30 societies. Unpublished manuscript.

Mackie, D. M., \& Smith, E. R. (2002). Intergroup emotions: Prejudice reconceptualized as differentiated reactions to out-groups. In J. P. Forgas \& K. D. Williams (Eds.), The social self: Cognitive, interpersonal, and intergroup perspectives (pp. 309 326). Philadelphia: Psychology Press.

Mackie, D. M., Devos, T., \& Smith, E. R. (2000). Intergroup emotions: Explaining offensive behavioral tendencies in an intergroup context. Journal of Personality and Social Psychology, 79, 602 - 616.

*Maltby, J., Day, L., \& Barber, L. (2004). Forgiveness and mental health variables. Interpreting the relationship using an adaptational-continuum model of personality and coping. Personality and Individual Differences, 37, 1629 - 1641.

*Maltby, J., Day, L., \& Barber, L. (2005). Forgiveness and happiness, the differing contexts of forgiveness using the distinction between hedonic and eudaimonic happiness. Journal of Happiness Studies, 6, 1 - 13.

Manzi, J., \& González, R. (2007). Forgiveness and Reparation in Chile. Peace and Conflict: Journal of Peace Psychology, 13, 71 - 92.

Margalit, A. (2005). To forgive and forget. In W. Edelstein \& G. Nunner-Winkler (Eds). Morality in Context (pp. 395-409). Amsterdam: Elsevier.

Marková, I. (2008). Social Identity and Social Representations: How are they related? In 
G. Moloney \& I. Walker (Eds.), Social representations and identity: Content, process and power (pp. 215 - 236). London: Palgrave Macmillan.

Markus, H. R., \& Kitayama, S. (1991). Culture and the self: Implications for cognition, emotion, and motivation. Psychological Review, 98, 224 - 253.

McCullough, M. E. (2000). Forgiveness as human strength: Theory, measurement, and links to well-being. Journal of Social \& Clinical Psychology. [Special Issue: Classical Sources of Human Strength: A Psychological Analysis], 19, 43 - 55.

McCullough, M. E. (2001). Forgiveness: Who does it and how do they do it? Current Directions in Psychological Science, 10, 194 - 197.

McCullough, M. E. (2008). Beyond revenge: The evolution of the forgiveness instinct. San Francisco: Jossey-Bass.

McCullough, M. E., \& Worthington, E. L., Jr. (1999). Religion and the Forgiving Personality. Journal of Personality, 67, 1141 - 1164.

McCullough, M. E., Bono, G., \& Root, L. M. (2007). Rumination, emotion, and forgiveness: Three longitudinal studies. Journal of Personality and Social Psychology, 92, 490 - 505.

McCullough, M. E., Fincham, F. D., \& Tsang, J.-A. (2003). Forgiveness, forbearance, and time: The temporal unfolding of transgression-related interpersonal motivations. Journal of Personality and Social Psychology, 84, 540 - 557.

McCullough, M. E., Thoresen, C. E., \& Pargament, K. I. (2000). Forgiveness: Theory, research, and practice. New York: Guilford Press.

McCullough, M. E., Worthington, E. L., \& Rachal, K. C. (1997). Interpersonal Forgiving in Close Relationships. Journal of Personality and Social Psychology, $73,321-336$.

McGlynn, C., Niens, U., Cairns, E., \& Hewstone, M. (2004). Moving out of conflict: the contribution of integrated schools in Northern Ireland to Identity, attitudes, forgiveness and reconciliation. Journal of Peace Education, 1, 147 - 163.

*McLernon, F., Cairns, E., Hewstone, M., \& Smith, R. (2004). The development of intergroup forgiveness in Northern Ireland. Journal of Social Issues. Special Issue: The Cost of Conflict: Children and the Northern Irish Troubles, 60, 587 601.

McLernon, F., Cairns, E., Lewis, C. A., \& Hewstone, M. (2003). Memories of recent 
conflict and forgiveness in Northern Ireland. In E. Cairns \& M. Roe (Eds.), The Role of Memory in Ethnic Conflict (pp. 125 - 143). London: Palgrave Macmillian.

Mellor, D., Bertherton, D., \& Firth, 1. (2007). Aboriginal and Non-Aboriginal Australia: The dilemma of apologies, forgiveness and reconciliation. Peace and Conflict: Journal of Peace Psychology, 13, 11 - 36.

Mendoza, K. R. (2003). Freeing the 'Slaves of Destiny': The Lolas of the Filipino Comfort Women movement. Cultural Dynamics, 15, 247 - 266.

Minow, M. (1998). Between vengeance and forgiveness: Facing history after genocide and mass killing. Boston: Beacon Press.

Mitchell, C. (2000). Gestures of conciliation: Factors contributing to successful olive branches. London: Macmillan Press.

Moeschberger, S. L., Dixon, D. N., Niens, U., \& Cairns, E. (2005). Forgiveness in Northern Ireland: Model for Peace in the Midst of the "Troubles". Peace \& Conflict: Journal of Peace Psychology, 11, 199 - 214.

Montada, L., Schmitt, M., \& Dalbert, C. (1986). Thinking about justice and dealing with one's own privileges: A study of existential guilt. In H. W. Bierhoff, R. Cohen \& J. Greenberg (Eds.), Justice in social relations (pp. 125 - 143). New York: Plenum Press.

Montiel, C. J. (2000). Constructive and Destructive Post-Conflict Forgiveness. Peace Review, 12, 95 - 101.

Montiel, C. J. (2002). Sociopolitical Forgiveness. Peace Review, 14, 271 - 277.

Morris-Suzuki, T., \& Rimmer, P. (2002). Virtual memories: Japanese history debates in manga and cyberspace, Asian Studies Review, 26, 147 - 164.

Moscovici, S. (1984). The phenomenon of social representations. In R. M. Farr \& S. Moscovici (Eds.), Social representations (pp. 3 - 70). Cambridge: Cambridge University Press.

Moscovici, S. (1988). Notes towards a description of social representations. European Journal of Social Psychology, 18, 211 - 250.

Moscovici, S. (1998). Social consciousness and its history. Culture andPsychology, 4, $411-429$.

Moscovici, S., \& Marková, I. (2000). Ideas and their development: A dialogue between 
Serge Moscovici and Ivana Marková. In S. Moscovici (edited by G. Duveen). Social Representations - Explorations in Social Psychology (pp. 224 - 286). Oxford: Blackwell Publishers Ltd.

Mullet, E., Girard, M., \& Bakhshi, P. (2004). Conceptualizations of forgiveness. European Psychologist, 9, 78 - 86.

Nadler, A., \& Liviatan, I. (2006). Intergroup reconciliation: Effects of adversary's expressions of empathy, responsibility, and recipient's trust. Personality and Social Psychology Bulletin, 32, 459 - 470.

Neto, F., Pinto, C., \& Mullet, E. (2007a). Seeking forgiveness in an intergroup context: Angolan, Guiean, Mozambican, and East Timorese perspectives. Regulation \& Governance, 1, $329-346$.

Neto, F., Pinto, C., \& Mullet, E. (2007b). Intergroup Forgiveness: East Timorese and Angolan Perspectives. Journal of Peace Research, 44, 711 - 729.

Noor, M., Brown, J. R., \& Prentice, G. (2008). Prospects for intergroup reconciliation: Social psychological predictors of intergroup forgiveness and reparation in Northern Ireland and Chile. In A. Nadler, T. Malloy \& J. D. Fisher (Eds.), Social Psychology of Inter-group Reconciliation: From violent conflict to peaceful coexistence - going beyond victimization, guilt and distrust (pp. 97 - 114). Oxford: Oxford University Press.

Noor, M., Brown, J. R., Gonzalez, R, Manzi, J \& Lewis, C. A. (2008). On positive psychological outcomes; what helps groups with a history of conflict to forgive and reconcile with each other? Personality and Social Psychology Bulletin, 34, $819-832$.

Noor, M., Brown, R., González, R., Manzi, J., \& Lewis, C. A. (2008). On Positive Psychological Outcomes: What Helps Groups With a History of Conflict to Forgive and Reconcile With Each Other? Personality and Social Psychology Bulletin, 34, 819 - 832.

Nozaki, Y. (2002). Japanese Politics and the History Textbook Controversy, 1982-2001, International Journal of Educational Research, 37, 603 - 622.

Ogawa, S. (2000). The difficulty of apology. Japan's struggle with memory and guilt. Harvard International Review, 22, 42.

Ohbuchi, K., \& Takahashi, Y. (1994). Cultural styles of conflict management in 
Japanese and Americans: passivity, covertness, and effectiveness of strategies. Journal of Applied Social Psychology, 24, 1345 - 1366.

Olick, J. K., \& Levy, D. (1997). Collective Memory and Cultural Constraint: Holocaust Myth and Rationality in German Politics. American Sociological Review, 62, $921-936$.

Oliner, S. P. (2005). Altruism, forgiveness, empathy, and intergroup apology. Humboldt Journal of Social Relations. [Special Issue: Altruism, intergroup apology and forgiveness: antidote for a divided world], 29, 8 - 40.

Oliner, S. P. (2008). Altruism, intergroup apology, forgiveness and reconciliation. St. Paul, MN: Paragon House.

*Orathinkal, J., Vansteenwegen, A., Enright, R. D., \& Stroobants, R. (2007). Further validation of the dutch version of the Enright Forgiveness Inventory. Community Mental Health Journal, 43, 109 - 128.

*Orcutt, H. K. (2006). The prospective relationship of interpersonal forgiveness and psychological distress symptoms among college women. Journal of Counseling Psychology, 53, 350 - 361.

*Orcutt, H. K., Pickett, S. M., \& Pope, E. B. (2005). Experiential avoidance and forgiveness as mediators in the relation between traumatic interpersonal events and posttraumatic stress disorder symptoms. Journal of Social \& Clinical Psychology, 24, 1003 - 1029.

Páez, D., \& Liu, J. H. (in press). Collective memory of conflicts. In D. Bar-Tal (Ed.), Intergroup Conflicts and their Resolution: Social Psychological Perspectives. New York: Psychology Press.

Páez, D., Marques, J., Valencia, J., \& Vincze, O. (2006). Dealing with collective guilt and shame. Psicología Política, 32, 59 - 78.

*Park, S. R. (1999). Measuring forgiveness across three cultures (Enright Forgiveness Inventory, United States, Korea, and Taiwan) (Doctoral Dissertation UMI, 1998). Dissertation Abstracts International Section A: Humanities and Social Sciences, 60, pp. 1451.

Pennebaker, J. W., Páez, D., \& Rimé, B. (1997). Collective Memory of Political Events. Mahwah, NJ: Lawrence Erlbaum.

Penney, M. (2008). Far from Oblivion: The Nanking Massacre in Japanese Historical 
Writing for Children and Young Adults. Holocaust and Genocide Studies, 22, 25 -48 .

Pettigrew, T. F. (1998). Intergroup contact theory. Annual Review of Psychology, 49, 65 -85 .

Pettigrew, T. F., \& Tropp, L. R. (2006). A meta-analytic test of intergroup contact theory. Journal of Personality and Social Psychology, 90, 751 - 783.

Poloma, M. M., \& Gallup, G. H. (1991). Varieties of prayer. Philadelphia: Trinity Press International.

Power, F. C. (2004). The moral self in community. In D. K. Lapsley \& D. Narvaez (Eds.), Moral development, self, and identity (pp. 47-64). Mahwah, NJ, US: Lawrence Erlbaum Associates Publishers.

Rata, A., Liu, J. H., \& Hanke, K. (2008). Te ara hohou rongo (the path to peace): Maori conceptualisations of inter-group forgiveness. New Zealand Journal of Psychology, 37, 18 - 30.

Raudenbush, S. W., \& Bryk, A. S. (2002). Hierarchical linear models: Applications and data analysis methods ( $2^{\text {nd }}$ ed.). Thousand Oaks, CA: Sage Publications.

Raudsepp, M. (2005). Why Is It So Difficult to Understand the Theory of Social Representations? Culture \& Psychology, 11, 455 - 468.

Reicher, S., \& Hopkins, N. (2001). Self and nation. London: Sage.

Reilly, J. (2006). China's history activism and sino-japanese relations. China: An International Journal, 4, 189 - 216.

Reischauer, E. O. (1988). The Japanese today: change and continuity. Cambridge, Mass.: Belknap Press.

Rensman, L. (2004). Collective guilt, national identity, and political processes in contemporary Germany. In N. R. Branscombe \& B. Doosje (Eds.), Collective Guilt: International Perspectives (pp. 169 - 192). Cambridge: University Press.

Ricouer, P. (2004). Memory, history, forgetting (K. Blamey \& D. Pellauer, Trans.). Chicago: University of Chicago Press.

*Rique, J. N. (1999). A cross-cultural study on the Enright Forgiveness Inventory: A measure for interpersonal forgiveness. Samples from Brazil and the United States. Unpublished Ph.D., The University of Wisconsin - Madison, United States -- Wisconsin. 
*Rique, J., Camino, C. P. S., Enright, R. D., \& Queiroz, P. (2007). Perdão interpessoal e contextos de injustiça no Brasil e Estados Unidos. / interpersonal forgiveness and the contexts of injustices in Brazil and the United States. PSICO, 38, 182 - 189.

*Rique, J., Enright, R. D., Serlin, R., \& Camino, C. P. S. (2007). Examining Interpersonal Forgiveness Across Seven Countries. Unpublished manuscript.

Roe, M. D. (2007). Intergroup Forgiveness in Settings of Political Violence: Complexities, Ambiguities, and Potentialities [Special Issue]. Peace \& Conflict: Journal of Peace Psychology, 13, 3 - 9.

Rosenberg, S. (2004). Face. In G. Burgess \& H. Burgess (Eds.) Beyond Intractability, Retrieved Sept 12, 2007 from <http://www.beyondintractability.org/essay/face> Conflict Research Consortium, University of Colorado, Boulder.

Rosoux, V. (2001). National Identity in France and Germany: from mutual exclusion to negotiation. International Negotiation, 6, 175-198.

Rothbart, M., \& John, O.P. (1985). Social categorization and behavioral episodes: a cognitive analysis of the effects of intergroup contact, Journal of Social Issues, $41,81-104$.

Ruchniewiecz, K. (2005). German War Compensation for Poland in the 1960s and 1970s, The Polish Foreign Affairs Digest, 1, 37 - 50.

Rye, M. S., Folck, C. D., Heim, T. A., Olszewski, B. T., \& Traina, E. (2004). Forgiveness of an ex-spouse: How does it relate to mental health following a divorce? Journal of Divorce \& Remarriage, 41, 31 - 51.

Sandage, S. J., \& Williams, I. (2005). Forgiveness in Cultural Context. In E. L. Worthington (Ed.), Handbook of forgiveness (pp. 41 - 55). New York: BrunnerRoutledge.

*Sarinopoulos, I. C. (1996). Forgiveness in adolescence and middle adulthood: Comparing the Enright forgiveness Inventory with wade Forgiveness Scale. University of Wisconsin-Madison.

*Sarinopoulos, I. C. (1999). Forgiveness and physical health. University of WisconsinMadison.

Schmitt, M., Behner, R., Montada, L., Müller, L., \& Müller-Fohrbrodt, G. (2000). Gender, ethnicity, and education as privileges: Exploring the generalizability of the existential guilt reaction. Social Justice Research, 13, 313 - 337. 
Schneider, C. (2008). The Japanese History Textbook Controversy in East Asian Perspective. The ANNALS of the American Academy of Political and Social Science, 617, 107 - 122.

Scobie, E. D. \& Scobie, G. E. W. (1998). Damaging Events: The Perceived Need for Forgiveness. Journal for the Theory of Social Behavior, 28, 373-401.

Shimazu, N. (2003). Popular Representations of the Past: The Case of Postwar Japan. Journal of Contemporary History, 38, 101 - 116.

Shriver, D. W. (1995). An ethic for enemies: Forgiveness in Politics. New York: Oxford University Press, Inc.

Shriver, D. W. (2005). Honest Patriots: loving a country enough to remember its misdeeds. Oxford: Oxford University Press.

Sibley, C. S., \& Liu, J. H. (2007). New Zealand = bicultural? Implicit and explicit associations between ethnicity and nationhood in the New Zealand context. European Journal of Social Psychology, 37, 1222 - 1243.

Sibley, C. S., Liu, J. H., Duckitt, J., \& Khan, S. S. (2008). Social representations of history and the legitimation of social inequality: The form and function of historical negation. European Journal of Psychology, 38, 542 - 565.

Smedes, L. B. (1984). Forgive and forget: Healing the hurt we don't deserve. New York: Harper \& Row.

Staerklé, C. (2006). The individual as the source of progressive thinking: a comment on Liu and Sibley (2006), Papers on Social Representations, 15, 6.1 -6.7.

Staub, E. (2000). Genocide and Mass Killing: Origins, Prevention, Healing and Reconciliation, Political Psychology, 21, 367 - 382.

Staub, E. (2004). Justice, healing, and reconciliation: How the people's courts in Rwanda can promote them. Peace and Conflict: Journal of Peace Psychology, $10,25-32$.

Staub, E. (2005). Constructive rather than harmful forgiveness, reconciliation and ways to promote them after genocide and mass killing. In E. L. Worthington, Jr. (Ed.). Handbook of forgiveness (pp. 443 - 459). New York: Taylor \& Francis Group.

Staub, E. (2006). Reconciliation after genocide, mass killing, or intractable conflict: Understanding the roots of violence, psychological recovery, and steps toward a general theory. Political Psychology, 27, 867-894. 
Staub, E., \& Bar-Tal, D. (2003). Genocide, Masskilling and Intractable Conflict. Roots, Evolution, Prevention and Reconciliation. In D. O. Sears, L. Huddy \& R. Jervis (Eds.), Oxford Handbook of Political Psychology (pp. 710 - 751). NY: Oxford Uni Press.

Staub, E., Pearlman, L. A., Gubin, A., \& Hagengimana, A. (2005) Healing, reconciliation, forgiving and the prevention of violence after genocide or mass killing: an intervention and its experimental evaluation in RWANDA, Journal of Social and Clinical Psychology, 24, 297 - 334.

Subkoviak, M. J., Enright, R. D., Wu, C., Gassin, E., Freedman, S., Olson, L., \&

*Sarinopoulos, I. (1995). Measuring interpersonal forgiveness in late adolescence and middle adulthood. Journal of Adolescence, 18, 641 - 655.

Tabachnick, B. G., \& Fidell, L. S. (2007). Using Multivariate Statistics (5 ed.). Boston: Pearson Education, Inc.

Tajfel, H., \& Turner, J. C. (1979). An integrative theory of intergroup conflict. In W. G. Austin \& S. Worchel (Eds.), The social psychology of intergroup relations (pp. 33-47). Monterey, CA: Brooks/Cole.

Tajfel, H., \& Turner, J. C. (1986). The social identity theory of intergroup behaviour. In S. Worchel \& W. G. Austin (Eds.), Psychology of intergroup relations (pp. 7 24). Chicago: Nelson-Hall.

Takahashi, C., Yamagishi, T., Liu, J. H., Wang, F. X., Lin, Y. C., \& Yu, S. H. (2008). The intercultural trust paradigm: Studying joint cultural interaction and social exchange in real time over the internet. International Journal of Intercultural Relations, 32, 215 - 228.

Takaku, S. (2001). The effects of apology and perspective taking on interpersonal forgiveness: A dissonance-attribution model of interpersonal forgiveness. The Journal of Social Psychology, 141, 494 - 508.

Takemoto, T., \& Ohara, Y. (2000). The Alleged "Nanking Massacre”. Japan's rebuttal to China's forged claims. Tôkyô: Meisei sha.

Tam, T., Hewstone, M., Cairns, E., Tausch, N., Maio, G., \& Kenworthy, J. (2007). The Impact of Intergroup Emotions on Forgiveness in Northern Ireland. Group Processes \& Intergroup Relations, 10, 119 - 136.

Tavuchis, N. (1991). Mea Culpa: A sociology of apology and reconciliation. Standford: 
Standford University Press.

Thelle, A. H. (2007). In the Shadows of the Atomic Holocaust - Japan's War-time Memories.I: Consilience: Centre for Advanced Study at the Norwegian Academy of Science and Letters 2007, 83 - 86.

Thomas, J. M., \& Garrod, A. (2002) Forgiveness after genocide? Perspectives from Bosnian youth. In S. Lamb \& J. G. Murphy (Eds) Before forgiving: Cautionary views of forgiveness in psychotherapy (pp. 192 - 211). New York: Oxford University Press.

Ting-Toomey, S. (2005) The Matrix of Face: An Updated Face-Negotiation Theory. In W.B. Gudykunst (Ed.), Theorizing About Intercultural Communication (pp.7192). Thousand Oaks, CA: Sage.

Toussaint, L. L., Williams, D. R., Musick, M. A. \& Everson, S. A. (2001). Forgiveness and health: Age differences in a U.S. probability sample. Journal of Adult Development, 8, 249 - 257.

Toussaint, L., \& Webb, J. R. (2005) Gender differences in the relationship between empathy and forgiveness. The Journal of Social Psychology, 145, 673 - 685.

*Toussaint, L., \& Webb, J. R. (2005). Gender differences in the relationship between empathy and forgiveness. Journal of Social Psychology, 145, 673 - 685.

Turner, J. C. (1987). A social categorization theory. In J. C. Turner, M. A. Hogg, P. J. Oakes, S. D. Reicher, and M. S. Wetherell (Eds.), Rediscovering the social group: A self-categorization theory. (pp. 42 - 67). Oxford: Blackwell.

Turner, J. C. (1999). Some current issues in research on social identity and selfcategorization theory. In N. Ellemers, R. Spears, and B. Doosje (Eds.), Social Identity (pp. 6-35). Oxford: Blackwell Publisher Ltd.

Turner, J. C., Hogg, M. A., Oakes, P. J., \& Wetherell, M. S. (1987). Rediscovering the social group: A self-categorization theory. Oxford: Blackwell.

Tutu, D. (2000). No Future Without Forgiveness. London: Random House.

United Nations Development Programme (2006). Summary. Human development report. Beyond scarcity: power, poverty and the global water crisis. New York: United Nations Development Programme.

van de Vijver, F., \& Leung, K. (1997). Methods and data analysis for cross-cultural research. Thousand Oaks: Sage Publication, Inc. 
van den Vliert, E., Schwartz, S. H., Huismans, S. E., Hofstede, G., \& Daan, S. (1999). Temperature, cultural masculinity, and domestic political violence: A crossnational study. Journal of Cross-Cultural Psychology, 30, 291 - 314.

van Hemert, D. A. (2003). Patterns of cross-cultural differences in psychology: A metaanalytical approach. Amsterdam: Dutch University Press.

van Hemert, D. A., Poortinga, Y. H., \& van de Vijver, F. J. R. (2007). Emotion and culture: A meta-analysis. Cognition \& Emotion, 21, 913 - 943.

Veenhoven, R. (1999). Quality-of-life in individualistic society A comparison of 43 nations in the early 90s. Social Indicators Research, 48(2), 157 - 186.

Viedt, S. (1993): Zwanzig Jahre Gemeinsame Deutsch-Polnische Schulbuchkommission. Reden der Festveranstaltung in Braunschweig am 10. Juni 1992. Braunschweig: Verlag Hahnsche Buchhandlung.

Vision of Humanity (2007). Global Peace Index -Report. Retrieved May 31, 2007 from http://www.visionofhumanity.org/gpi/documents/results-report.php

Vision of Humanity (2008). Global Peace Index -Report. Retrieved April 1, 2009 from http://www.visionofhumanity.org/gpi/documents/results-report.php

Vision of Humanity (2009). Global Peace Index -Report. Retrieved April 1, 2009 from http://www.visionofhumanity.org/gpi/documents/results-report.php

Voelklein, C., \& Howarth, C. (2005). A review of controversies about social representations theory: a British debate, Culture \& Psychology, 11(4), 431 - 454.

von Borries, B. (2003). The Third Reich in German History Textbooks since 1945. Journal of Contemporary History, 38(1), 45 - 62.

Wagner, W, \& Hayes, N. (2005). Everyday Discourse and Common Sense. The Theory of Social Representations. NY: Palgrave Macmillan.

*Waltman, M., Lin, W., Wee, D., \& Engstrand, E. A. (1999). Measuring interpersonal forgiveness in high school students. In J. Rique, M. Waltman, I. Sarinopoulos, W. Lin, D. Wee, E. A. Engstrand and R. D. Enright, The tools of forgiveness education, (In Press).

Wertsch, J. V. (2002). Voices of collective remembering. Cambridge: Cambridge University Press.

Wohl, M. J. A., \& Branscombe, N. R. (2005). Forgiveness and collective guilt assignment to historical perpetrator groups depend on level of social category 
inclusiveness. Journal of Personality and Social Psychology, 88, 288 - 303.

Wohl, M. J. A., \& Reeder, G. D. (2004). When bad deeds are forgiven: Judgments of morality and forgiveness for intergroup aggression. In J. P. Morgan (Ed.), Focus on Aggression Research (pp. 59-74). New York: Nova Science Publishers, Inc.

Worthington, E. L. (Ed.). (2005). Handbook of forgiveness. New York: BrunnerRoutledge.

Yamazaki, J. W. (2006). Japanese Apologies for World War II: A Rhetorical Study. London and New York: Routledge.

Yamazaki, J. W. (2006). Japanese apologies for World War: a rhetorical study. London: Routledge.

Zechmeiser, J. S., Garcia, S., Romero, C., \& Vas, S. N. (2004). Don’t apologize unless you mean it: A laboratory investigation of forgiveness and retaliation. Journal of Social and Clinical Psychology, 23, 532 - 564. 


\section{Appendices}

\section{Appendix A - Semi-structured interview schedule}

\section{Part 1: Introduction to the study (approx. 10 min)}

Introduction (state objective of the study, procedure of the interview and ground rules, questions from the participant regarding the research)

\section{Part 2: Interview (approx. 60-90min)}

Demographic questions (5-10min)

1. In which country do you live now?

2. In which country were you born?

3. With which religious or philosophical orientation do you most identify?

4. What is your age?

\section{Forgiveness (individual level) $\mathbf{( 1 5 - 2 0 \mathrm { min } )}$}

1. Can you describe a situation where you felt unjustly hurt or harmed?

2. Can you describe a situation or an experience where forgiveness was granted by you?

3. When thinking of these situations, what does forgiveness mean to you?

4. What do you think is the value of forgiveness?

Forgiveness (group level) (20-30min)

1. Has anything happened in the past to your national or ethnic group that might require forgiveness? Please describe what this might be.

2. What does forgiveness mean to you in such a context?

3. Do you think "intergroup forgiveness" is possible?(probe: why or why not)

4. Do you think "intergroup forgiveness" is important? (probe: In what ways might it be important?)

5. In your opinion what is the difference between individual forgiveness and forgiveness between groups?

6. What do you think about the relationship between forgiveness and reconciliation between groups?

\section{Experiences (20-30min)}

1. Did you ever experience of any situations where you felt insulted, hurt or harmed on behalf of your nation/ethnicity? Please describe such a situation. (group)

2. In what context did this experience/situation take place? (Probe: For example when you read the newspaper? When you listened to a political speech? When you learnt about historical wrong doings done by another nation/ethnic group to your nation/ethnic group? When you had or listened to a discussion?)

3. How did you feel? (emotions)

4. What did you do? (behaviour)

5. What did you think? (cognitive)

6. In your opinion, what would have made you feel better?

7. In your opinion, who should be the one to apologize for past wrong doings? (probe: representatives, leaders?)

8. In your opinion: what makes a public apology sincere?

Part 3: Post interview/post discussion (saying thank you, reiterating confidentiality and debriefing) (approx. $10 \mathrm{~min}$ ) 


\section{Appendix B - Additional Information for Chapter 5}

Appendix B1 - Survey Study 3 European Victim Version (English wording only)
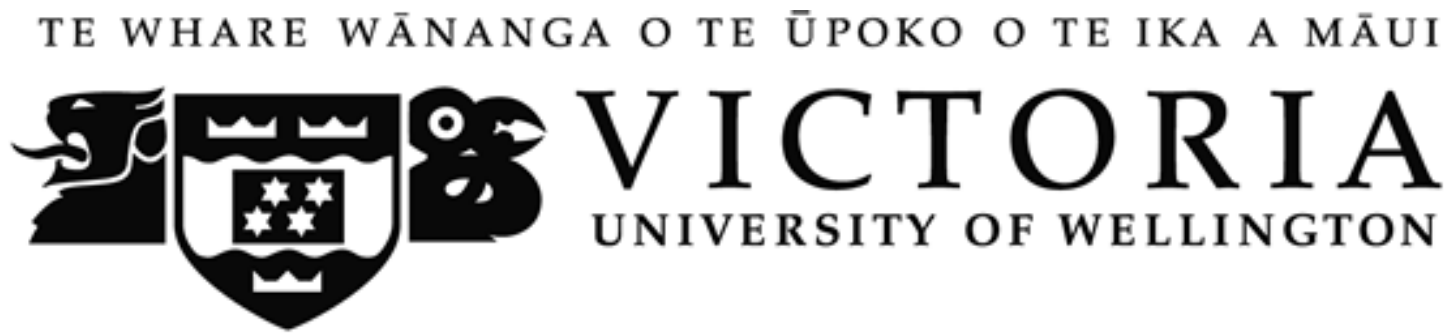

Dear participant,

You are invited to participate in an international survey on your views and perceptions of current relations between Germany and her European neighbours.

We would appreciate it, if you would be willing to fill out the following questionnaire. Your participation is entirely voluntary and you are free to withdraw at any stage before you complete and hand back the questionnaire to us. You grant your consent to participate in this research by filling in the questionnaire.

On the following pages, you will see some questions about some general and specific statements about politics and international relations. Please answer these questions as honestly as possible using the provided rating scales. It will take about 30 minutes to fill out the questionnaire.

- Your answers are completely anonymous and only investigators directly involved in the project will have access to the data.

- You will never be personally identified in this research project or in any presentation or publication.

- The information you provide will be coded by number only. In accordance with the requirements of some scientific journals and organisations, your coded data may be shared with other competent researchers.

- Your coded data may be used in other, related studies.

- A copy of the coded data will remain in the custody of investigators.

Please do not write your name or any identifying information on the questionnaire itself.

The data you provide may be used for one or more of the following purposes:

- The overall findings may be submitted for publication in a scientific journal, or presented at scientific conferences.

- The overall findings may form part of a PhD thesis that will be submitted for assessment.

Please remember, there are no right or wrong answers. We are interested in your personal opinion on these issues.

Thank you very much for your help and cooperation.

Katja Hanke

PhD Student

Victoria University of Wellington

School of Psychology

PO Box 600

Wellington

New Zealand

Email:katja.hanke@vuw.ac.nz
Assoc. Prof. James H. Liu

Associate Professor

Victoria University of Wellington

School of Psychology

PO Box 600

Wellington

New Zealand

Email:james.liu@vuw.ac.nz 


\section{Section A: Identity}

Please indicate to which degree you disagree (1) or agree (7) with each of the following statements. There are no right or wrong answers. The best answer is your own opinion.

\begin{tabular}{|c|c|c|c|c|c|c|c|}
\hline National Identity & \multicolumn{3}{|c|}{$\begin{array}{l}\text { Strongly } \\
\text { disagree } \\
\Downarrow\end{array}$} & \multicolumn{2}{|c|}{$\begin{array}{c}\text { Neutral } \\
\Downarrow\end{array}$} & \multicolumn{2}{|c|}{$\begin{array}{r}\text { Strongly } \\
\text { agree } \\
\Downarrow \\
\end{array}$} \\
\hline 1. I feel a bond with [members of your country]. & 1 & 2 & 3 & 4 & 5 & 6 & 7 \\
\hline 2. I feel solidarity with [members of your country]. & 1 & 2 & 3 & 4 & 5 & 6 & 7 \\
\hline 3. I feel committed to [members of your country]. & 1 & 2 & 3 & 4 & 5 & 6 & 7 \\
\hline 4. I am glad to be [your nationality]. & 1 & 2 & 3 & 4 & 5 & 6 & 7 \\
\hline $\begin{array}{l}\text { 5. I think that [members f your country] have a lot to be } \\
\text { proud of. }\end{array}$ & 1 & 2 & 3 & 4 & 5 & 6 & 7 \\
\hline 6. It is pleasant to be [your nationality]. & 1 & 2 & 3 & 4 & 5 & 6 & 7 \\
\hline 7. I often think about the fact that I am [your nationality]. & 1 & 2 & 3 & 4 & 5 & 6 & 7 \\
\hline $\begin{array}{l}\text { 8. The fact that I am [your nationality] is an important part of } \\
\text { my identity. }\end{array}$ & 1 & 2 & 3 & 4 & 5 & 6 & 7 \\
\hline $\begin{array}{l}\text { 9. Being [your nationality] is an important part of how I see } \\
\text { myself. }\end{array}$ & 1 & 2 & 3 & 4 & 5 & 6 & 7 \\
\hline 10. I am similar to many other [members of your country]. & 1 & 2 & 3 & 4 & 5 & 6 & 7 \\
\hline $\begin{array}{l}\text { 11. [Members of your country] have a lot in common with } \\
\text { each other. }\end{array}$ & 1 & 2 & 3 & 4 & 5 & 6 & 7 \\
\hline 12. [Members of your country] are very similar to each other. & 1 & 2 & 3 & 4 & 5 & 6 & 7 \\
\hline
\end{tabular}

Section B: Perception of history

We are interested in your perception of the consequences of historical events on current relations between Germany and [your country]. On the following pages, you will be asked a number of questions about consequences, perceptions, emotions and thoughts about Hitler's rule in Germany and Germany's war-time past.

Please answer these questions as honestly as possible using the provided rating scales. There are no right or wrong answers. The best answer is your own opinion.

\section{PERCEPTION OF HISTORY}

Please indicate to which degree you disagree (1) or agree (7) with each of the following statements.

1. Discussions about apologizing and forgiving related to Germany's past war crimes are not important any more.

2. Grievances and resentment related to Germany's past war crimes will be carried to the next generation.

3. Negative feelings due to Germany's past war crimes have faded with time.

4. Germany's past war crimes are still influencing the relationship between Germany and [your country].

5. Today there is no need to talk much about the harm committed by Germany in the past.

6. [Your nationality] stories of surviving Germany's past war crimes make me feel like the wounds are still open today.

\begin{tabular}{|c|c|c|c|c|c|c|}
\hline $\begin{array}{l}\text { Strongly } \\
\text { disagree } \\
\Downarrow\end{array}$ & \multicolumn{4}{|c}{$\begin{array}{r}\text { Neutral } \\
\Downarrow\end{array}$} & \multicolumn{3}{r|}{$\begin{array}{r}\text { Strongly } \\
\text { agree } \\
\Downarrow\end{array}$} \\
\hline 1 & 2 & 3 & 4 & 5 & 6 & 7 \\
\hline 1 & 2 & 3 & 4 & 5 & 6 & 7 \\
\hline 1 & 2 & 3 & 4 & 5 & 6 & 7 \\
\hline 1 & 2 & 3 & 4 & 5 & 6 & 7 \\
\hline 1 & 2 & 3 & 4 & 5 & 6 & 7 \\
\hline 1 & 2 & 3 & 4 & 5 & 6 & 7 \\
\hline
\end{tabular}




\section{Section C: Emotions \& Thoughts}

The following statements are $s$ about the relation between past misdeeds of Germany and Germans today.

Please indicate the degree to which you disagree (' 1 ') or agree ('7') with each statement. There are no right or wrong answers. The best answer is your own opinion.

\section{GUILT ASSIGNMENT}

1. Today's Germans should feel regret for the harm done in the past towards other groups.

2. Today's Germans should feel guilty about the negative things their ancestors did to other groups in the past.

3. Today's Germans should feel regret for some of the things their ancestors did to other groups in the past.

4. Today's Germans should repair damage caused to other groups by their ancestors.

5. Today's Germans should easily feel guilty for the bad outcomes brought about by their ancestors.

\begin{tabular}{|c|c|c|c|c|c|c|}
\hline \multicolumn{3}{|c}{$\begin{array}{l}\text { Strongly } \\
\text { disagree } \\
\Downarrow\end{array}$} & \multicolumn{3}{|r}{$\begin{array}{r}\text { Neutral } \\
\Downarrow\end{array}$} & $\begin{array}{r}\text { Strongly } \\
\text { agree } \\
\Downarrow\end{array}$ \\
\hline 1 & 2 & 3 & 4 & 5 & 6 & 7 \\
\hline 1 & 2 & 3 & 4 & 5 & 6 & 7 \\
\hline 1 & 2 & 3 & 4 & 5 & 6 & 7 \\
\hline 1 & 2 & 3 & 4 & 5 & 6 & 7 \\
\hline 1 & 2 & 3 & 4 & 5 & 6 & 7 \\
\hline
\end{tabular}

\section{EMOTIONS}

Please indicate to which degree from 'not at all' (1) to '7' (extremely) you experience these emotions when thinking of Germany's handling of the past misdeeds.

\begin{tabular}{|c|c|c|c|c|c|c|c|}
\hline of the past misdeeds. & at al & & & nev & & & nely \\
\hline $\begin{array}{l}\text { How Germany has dealt with its past misdeeds during World War } \\
\text { II makes me feel... }\end{array}$ & $\Downarrow$ & & & $\Downarrow$ & & & $\Downarrow$ \\
\hline 1. ...angry & 1 & 2 & 3 & 4 & 5 & 6 & 7 \\
\hline 2. ...calm & 1 & 2 & 3 & 4 & 5 & 6 & 7 \\
\hline 3. ...anxious & 1 & 2 & 3 & 4 & 5 & 6 & 7 \\
\hline 4. ...cheerful & 1 & 2 & 3 & 4 & 5 & 6 & 7 \\
\hline 5. ...displeased & 1 & 2 & 3 & 4 & 5 & 6 & 7 \\
\hline 6. ...afraid & 1 & 2 & 3 & 4 & 5 & 6 & 7 \\
\hline 7. ...fearful & 1 & 2 & 3 & 4 & 5 & 6 & 7 \\
\hline 8. ...happy & 1 & 2 & 3 & 4 & 5 & 6 & 7 \\
\hline 9. ...furious & 1 & 2 & 3 & 4 & 5 & 6 & 7 \\
\hline 10. ...irritated & 1 & 2 & 3 & 4 & 5 & 6 & 7 \\
\hline 11. ...worried & 1 & 2 & 3 & 4 & 5 & 6 & 7 \\
\hline 12. ...pleased & 1 & 2 & 3 & 4 & 5 & 6 & 7 \\
\hline
\end{tabular}




\begin{tabular}{|c|c|c|c|c|c|c|c|}
\hline \multirow{2}{*}{$\begin{array}{l}\text { FORGIVENESS } \\
\text { You may have read in the newspapers, or heard/seen discussions } \\
\text { in the mass media about apologies and attempts of } \\
\text { rapprochement between Germany and [your country]. } \\
\text { Please tell us how you feel about following statements and indicate to } \\
\text { which degree you disagree ('1) or agree (' } 7 \text { '). } \\
\begin{array}{l}\text { It is important that [your country] never forgives the past harm } \\
\text { done during Hitler's rule and World War II by Germany. }\end{array}\end{array}$} & \multicolumn{3}{|c|}{$\begin{array}{l}\text { Strongly } \\
\text { disagree } \\
\Downarrow\end{array}$} & \multicolumn{2}{|c|}{$\underset{\Downarrow}{\text { Neutral }}$} & \multicolumn{2}{|c|}{$\begin{array}{r}\text { Strongly } \\
\text { agree } \\
\Downarrow\end{array}$} \\
\hline & 1 & 2 & 3 & 4 & 5 & 6 & 7 \\
\hline $\begin{array}{l}\text { [Your country] has remained strong precisely because they } \\
\text { have never forgiven past war crimes committed by Germany. }\end{array}$ & 1 & 2 & 3 & 4 & 5 & 6 & 7 \\
\hline $\begin{array}{l}\text { 3. Forgiving Germany for past war crimes would be a betrayal to } \\
\text { former victims. }\end{array}$ & 1 & 2 & 3 & 4 & 5 & 6 & 7 \\
\hline $\begin{array}{l}\text { 4. Today's Germans should be forgiven for what their group did } \\
\text { [members of your country] during Hitler's rule and World War } \\
\text { II. }\end{array}$ & 1 & 2 & 3 & 4 & 5 & 6 & 7 \\
\hline $\begin{array}{l}\text { [Your country] should move past their negative feelings } \\
\text { towards today's Germans for the harm their group inflicted } \\
\text { during Hitler's rule and World War II. }\end{array}$ & 1 & 2 & 3 & 4 & 5 & 6 & 7 \\
\hline $\begin{array}{l}\text { 6. Today's Germans should be forgiven for what their ancestors } \\
\text { did [members of your country] during Hitler's rule and World } \\
\text { War II. }\end{array}$ & 1 & 2 & 3 & 4 & 5 & 6 & 7 \\
\hline $\begin{array}{l}\text { 7. It is possible for me to forgive today's Germans for Germany's } \\
\text { past war crimes. }\end{array}$ & 1 & 2 & 3 & 4 & 5 & 6 & 7 \\
\hline $\begin{array}{l}\text { The following statements are statements about granting } \\
\text { forgiveness. }\end{array}$ & $\begin{array}{l}\text { Stron } \\
\text { disag } \\
\Downarrow\end{array}$ & & & $\underset{\Downarrow}{\text { Neutr }}$ & & & \\
\hline $\begin{array}{l}\text { 1. Granting forgiveness will put the victimized group into an even } \\
\text { more vulnerable position than before. }\end{array}$ & 1 & 2 & 3 & 4 & 5 & 6 & 7 \\
\hline $\begin{array}{l}\text { 2. Granting forgiveness without empowering the victimized group } \\
\text { can lead to a loss of identity for the victimized group. }\end{array}$ & 1 & 2 & 3 & 4 & 5 & 6 & 7 \\
\hline 3. Granting forgiveness is an invitation for crimes to be repeated. & 1 & 2 & 3 & 4 & 5 & 6 & 7 \\
\hline 4. Granting forgiveness means losing sympathy as victims. & 1 & 2 & 3 & 4 & 5 & 6 & 7 \\
\hline $\begin{array}{l}\text { 5. Granting forgiveness will make the perpetrator believe that now } \\
\text { everything is alright although nothing is really settled. }\end{array}$ & 1 & 2 & 3 & 4 & 5 & 6 & 7 \\
\hline $\begin{array}{l}\text { TRUST } \\
\text { Please tell us how you feel about interactions between Germans } \\
\text { and [members of your country]. Please indicate to which degree } \\
\text { from 'not at all' (1) to 'very much' (7) you think each of these } \\
\text { question are true. }\end{array}$ & $\begin{array}{l}\text { Not } \\
\text { at all } \\
\Downarrow\end{array}$ & & & 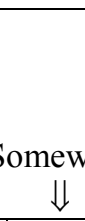 & & & $\begin{array}{r}\text { Very } \\
\text { Much } \\
\Downarrow\end{array}$ \\
\hline $\begin{array}{l}\text { Do you think today's Germans would try to be fair to [members } \\
\text { of your country]? }\end{array}$ & 1 & 2 & 3 & 4 & 5 & 6 & 7 \\
\hline $\begin{array}{l}\text { 2. Would you say that most of the time today's Germans try to be } \\
\text { helpful to [members of your country]? }\end{array}$ & 1 & 2 & 3 & 4 & 5 & 6 & 7 \\
\hline $\begin{array}{l}\text { 3. Generally speaking, would you say that most today's Germans } \\
\text { can be trusted? }\end{array}$ & 1 & 2 & 3 & 4 & 5 & 6 & 7 \\
\hline
\end{tabular}


1. Are you? $\square$ female $\quad \square$ male

2. What is your age? years

3. What is your religion (if any)? Please specify:

3a. How important is religion in your life? (please circle a number between 1 and 4 to describe how you feel)

$1=$ not important at all, $2=$ somewhat important, $3=$ fairly important, $4=$ very important

4. Do you actively practice a religion (e.g. going to church, temple)? $\square$ yes $\square$ no

4a. If yes, how often do you practice your religion? Please circle a number between 1 (very seldom) and 7 (daily) that best describes you:
(very seldom) 1
2
3
45
67 (daily)

5. What is your highest form of education?

$\square$ Secondary school

$\square$ Apprenticeship

$\square$ Polytechnic

$\square$ University

6. What is your occupation?

7. What is your Country of Birth?

8. What is your Nation of Citizenship(s)?

9. How long have you lived in countries outside of this one? years months

10. Does your family have a migration background? $\square$ yes; $\square$ no; $\square$ partially

a. If yes, from which country did your family or parts of your family come from?

b. How long have they been living in [country where participant live in]?____ years ___ months

11. Often, when people talk about political matters they use terms like "right-wing" or "leftwing" to describe their views. How would you describe yourself in these terms? Circle a number between 1 (left-wing) and 7 (right-wing) that best describes you:

$\begin{array}{lllllllll}\text { (left-wing) } & 1 & 2 & 3 & 4 & 5 & 6 & 7 \text { (right-wing) }\end{array}$

a) Other political orientation, please specify:

Thanks a lot for your participation and your help in this research! 


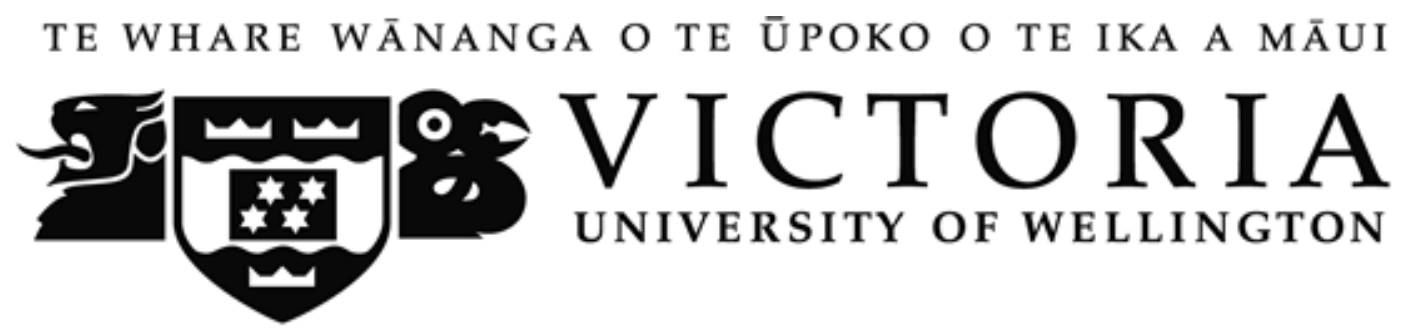

\section{Debriefing}

Thank you very much for taking the time and effort to complete this questionnaire and for participating in this research.

The study examines what promotes and hinders forgiveness between once conflicting countries.

Forgiveness between countries may be an alternative means to improve relations between countries that used to be in conflicts. Previous studies have shown that feeling guilty on behalf of your country influences people's willingness to try to repair damage that was caused in the past. Furthermore, empathy (the ability to take another perspective) and trust (the expectation to be treated fairly by another person or group) have an impact on the willingness to forgive. However, forgiveness between countries is still poorly understood. More studies are needed that relate to conflicts that actually happened in order to discover pragmatic means that can improve relations between countries.

In this study we attempted to look at a real post conflict situation between countries and examine forgiveness from a cultural comparison perspective. This has been neglected in previous research. Thus, this perspective can contribute to our understanding about rapprochement between countries after conflict.

Preliminary results of this study will be posted on the website of the Centre for Applied Cross-Cultural Research (http://www.vuw.ac.nz/cacr/) by March 2009. If you have any additional questions please feel free to contact Katja Hanke at Katja.Hanke@ vuw.ac.nz

Thank you very much again for participating in this study. 


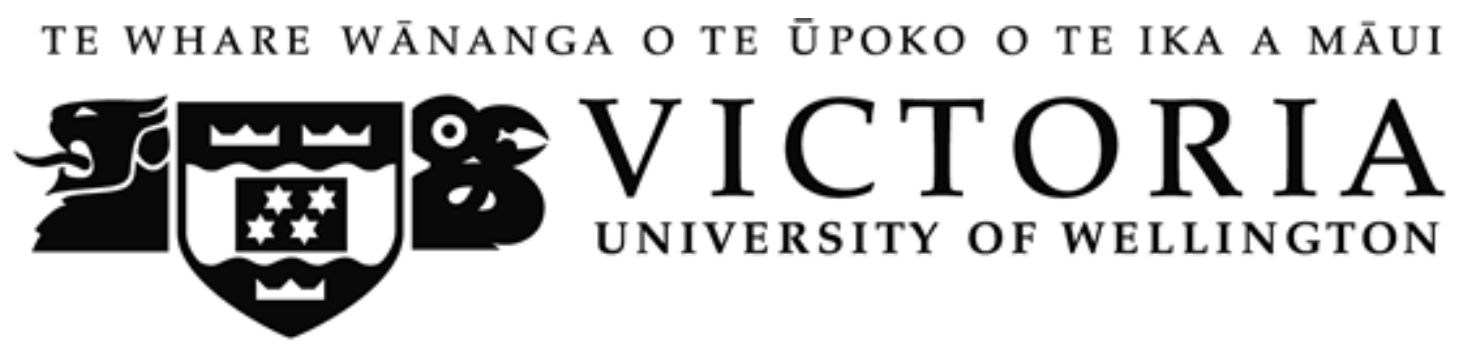

Dear participant,

You are invited to participate in an international survey on your views and perceptions of current relations between Japan and her Asian neighbours.

We would appreciate it, if you would be willing to fill out the following questionnaire. Your participation is entirely voluntary and you are free to withdraw at any stage before you complete and hand back the questionnaire to us. You grant your consent to participate in this research by filling in the questionnaire.

On the following pages, you will see some questions about some general and specific statements about politics and international relations. Please answer these questions as honestly as possible using the provided rating scales. It will take about 30 minutes to fill out the questionnaire.

- Your answers are completely anonymous and only investigators directly involved in the project will have access to the data.

- You will never be personally identified in this research project or in any presentation or publication.

- The information you provide will be coded by number only. In accordance with the requirements of some scientific journals and organisations, your coded data may be shared with other competent researchers.

- Your coded data may be used in other, related studies.

- A copy of the coded data will remain in the custody of investigators.

Please do not write your name or any identifying information on the questionnaire itself.

The data you provide may be used for one or more of the following purposes:

- The overall findings may be submitted for publication in a scientific journal, or presented at scientific conferences.

- The overall findings may form part of a PhD thesis that will be submitted for assessment.

Please remember, there are no right or wrong answers. We are interested in your personal opinion on these issues.

Thank you very much for your help and cooperation.

Katja Hanke

PhD Student

Victoria University of Wellington

School of Psychology

PO Box 600

Wellington

New Zealand

Email:katja.hanke@vuw.ac.nz
Assoc. Prof. James H. Liu

Associate Professor

Victoria University of Wellington

School of Psychology

PO Box 600

Wellington

New Zealand

Email: james.liu@vuw.ac.nz 


\section{Section A: Identity}

Please indicate to which degree you disagree (1) or agree (7) with each of the following statements.

There are no right or wrong answers. The best answer is your own opinion.

\begin{tabular}{|c|c|c|c|c|c|c|c|}
\hline National Identity & $\begin{array}{l}\text { Stro } \\
\text { disa } \\
\Downarrow\end{array}$ & & & & & & $\begin{array}{r}\text { gly } \\
\text { ree } \\
\Downarrow \\
\end{array}$ \\
\hline 1. I feel a bond with [members of your country]. & 1 & 2 & 3 & 4 & 5 & 6 & 7 \\
\hline 2. I feel solidarity with [members of your country]. & 1 & 2 & 3 & 4 & 5 & 6 & 7 \\
\hline 3. I feel committed to [members of your country]. & 1 & 2 & 3 & 4 & 5 & 6 & 7 \\
\hline 4. I am glad to be [your nationality]. & 1 & 2 & 3 & 4 & 5 & 6 & 7 \\
\hline $\begin{array}{l}\text { 5. I think that [members of your country] have a lot to be proud } \\
\text { of. }\end{array}$ & 1 & 2 & 3 & 4 & 5 & 6 & 7 \\
\hline 6. It is pleasant to be [your nationality]. & 1 & 2 & 3 & 4 & 5 & 6 & 7 \\
\hline 7. I often think about the fact that I am [your nationality]. & 1 & 2 & 3 & 4 & 5 & 6 & 7 \\
\hline $\begin{array}{l}\text { 8. The fact that I am [your nationality] is an important part of my } \\
\text { identity. }\end{array}$ & 1 & 2 & 3 & 4 & 5 & 6 & 7 \\
\hline $\begin{array}{l}\text { 9. Being [your nationality] is an important part of how I see } \\
\text { myself. }\end{array}$ & 1 & 2 & 3 & 4 & 5 & 6 & 7 \\
\hline 10. I am similar to many other [members of your country]. & 1 & 2 & 3 & 4 & 5 & 6 & 7 \\
\hline $\begin{array}{l}\text { 11. [Members of your country] have a lot in common with each } \\
\text { other. }\end{array}$ & 1 & 2 & 3 & 4 & 5 & 6 & 7 \\
\hline 12. [Members of your country] are very similar to each other. & 1 & 2 & 3 & 4 & 5 & 6 & 7 \\
\hline
\end{tabular}

\section{Section B: Perception of history}

We are interested in your perception of the consequences of historical events on current relations between Japan and [your country]. On the following pages, you will be asked a number of questions about consequences, perceptions, emotions and thoughts about Japan's colonial and war-time past.

Please answer these questions as honestly as possible using the provided rating scales. There are no right or wrong answers. The best answer is your own opinion.

\section{PERCEPTION OF HISTORY}

Please indicate to which degree you disagree (1) or agree (7) with each of the following statements.

\begin{tabular}{|c|c|c|c|c|c|c|c|c|}
\hline 1. & $\begin{array}{l}\text { Discussions about apologizing and forgiving related to Japan's } \\
\text { harmful past actions towards [members of your country] are not } \\
\text { important any more. }\end{array}$ & 1 & 2 & 3 & 4 & 5 & 6 & 7 \\
\hline 2. & $\begin{array}{l}\text { Grievances and resentment related to Japan's harmful past } \\
\text { actions towards [members of your country] will be carried to the } \\
\text { next generation. }\end{array}$ & 1 & 2 & 3 & 4 & 5 & 6 & 7 \\
\hline 3. & $\begin{array}{l}\text { Negative feelings due to Japan's harmful past actions towards } \\
\text { [members of your country] have faded with time. }\end{array}$ & 1 & 2 & 3 & 4 & 5 & 6 & 7 \\
\hline 4. & $\begin{array}{l}\text { Japan's harmful past actions towards [members of your country] } \\
\text { are still influencing the relationship between Japan and [your } \\
\text { country]. }\end{array}$ & 1 & 2 & 3 & 4 & 5 & 6 & 7 \\
\hline 5. & $\begin{array}{l}\text { Today there is no need to talk so much about the harm committed } \\
\text { by Japan in the past. }\end{array}$ & 1 & 2 & 3 & 4 & 5 & 6 & 7 \\
\hline 6. & $\begin{array}{l}\text { [Your nationality] stories of surviving Japan's harmful past } \\
\text { actions make me feel like the wounds are still open today. }\end{array}$ & 1 & 2 & 3 & 4 & 5 & 6 & 7 \\
\hline
\end{tabular}




\section{Section C: Emotions \& Thoughts}

The following statements are about the relation between past misdeeds of Japan and Japanese people today Please indicate to which degree you disagree (1) or agree (7) with each of the following statements. There are no right or wrong answers. The best answer is your own opinion.

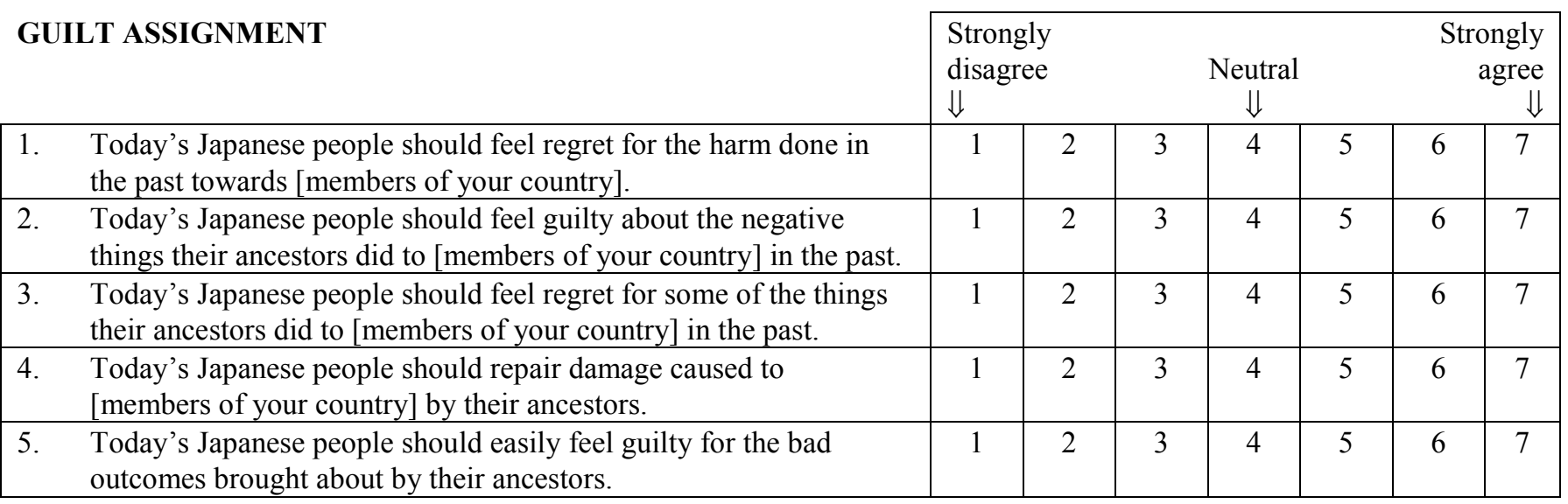

\section{EMOTIONS}

Please indicate to which degree from 'not at all' (1) to 'extremely' (7) you experience these emotions when thinking of Japan's handling of the past misdeeds.

\begin{tabular}{|c|c|c|c|c|c|c|c|}
\hline \multirow[b]{2}{*}{$\begin{array}{l}\text { How Japan has dealt with its past misdeeds during the occupation of } \\
\text { Asia, the Sino-Japanese War and World War II makes me feel... }\end{array}$} & \multicolumn{3}{|l|}{ at al } & & \\
\hline & $\Downarrow$ & & & $\Downarrow$ & & & $\Downarrow$ \\
\hline 1. ...angry & 1 & 2 & 3 & 4 & 5 & 6 & 7 \\
\hline 2. $\quad$...calm & 1 & 2 & 3 & 4 & 5 & 6 & 7 \\
\hline 3. $\quad$...anxious & 1 & 2 & 3 & 4 & 5 & 6 & 7 \\
\hline ...cheerful & 1 & 2 & 3 & 4 & 5 & 6 & 7 \\
\hline 5. $\quad$...displeased & 1 & 2 & 3 & 4 & 5 & 6 & 7 \\
\hline 6. ...afraid & 1 & 2 & 3 & 4 & 5 & 6 & 7 \\
\hline 7. ...fearful & 1 & 2 & 3 & 4 & 5 & 6 & 7 \\
\hline ...happy & 1 & 2 & 3 & 4 & 5 & 6 & 7 \\
\hline 9. ...furious & 1 & 2 & 3 & 4 & 5 & 6 & 7 \\
\hline 10. ...irritated & 1 & 2 & 3 & 4 & 5 & 6 & 7 \\
\hline 11. ...worried & 1 & 2 & 3 & 4 & 5 & 6 & 7 \\
\hline 12. ...pleased & 1 & 2 & 3 & 4 & 5 & 6 & 7 \\
\hline
\end{tabular}




\begin{tabular}{|c|c|c|c|c|c|c|c|}
\hline $\begin{array}{l}\text { FORGIVENESS } \\
\text { You may have read in the newspapers, or heard/seen discussions in the } \\
\text { mass media about apologies and attempts of rapprochement between } \\
\text { Japan and [your country]. } \\
\text { Please tell us how you feel about following statements and indicate to } \\
\text { which degree you disagree (' } 1 \text { ') or agree (' } 7 \text { '). }\end{array}$ & $\begin{array}{l}\text { Stron } \\
\text { disag } \\
\Downarrow\end{array}$ & & & $\begin{array}{c}\text { Neutra } \\
\Downarrow\end{array}$ & & & $\begin{array}{r}\text { ngly } \\
\text { gree } \\
\Downarrow \\
\end{array}$ \\
\hline $\begin{array}{l}\text { 1. It is important that [your country] never forgives the past harm done } \\
\text { during the occupation of Asia, the Sino-Japanese War and World War } \\
\text { II by Japan. }\end{array}$ & 1 & 2 & 3 & 4 & 5 & 6 & 7 \\
\hline $\begin{array}{l}\text { 2. [Your country] has remained strong precisely because they have } \\
\text { never forgiven past misdeeds committed by Japan. }\end{array}$ & 1 & 2 & 3 & 4 & 5 & 6 & 7 \\
\hline $\begin{array}{l}\text { 3. Forgiving Japan for past war crimes would be a betrayal to former } \\
\text { victims. }\end{array}$ & 1 & 2 & 3 & 4 & 5 & 6 & 7 \\
\hline $\begin{array}{l}\text { 4. Today's Japanese should be forgiven for what their group did to } \\
\text { members your country] during Japan's period of colonisation and } \\
\text { warfare. }\end{array}$ & 1 & 2 & 3 & 4 & 5 & 6 & 7 \\
\hline $\begin{array}{l}\text { [Your country] should move past their negative feelings towards } \\
\text { today's Japanese for the harm their group inflicted during the } \\
\text { occupation of Asia, the Sino-Japanese War and World War II. }\end{array}$ & 1 & 2 & 3 & 4 & 5 & 6 & 7 \\
\hline $\begin{array}{l}\text { 6. Today's Japanese should be forgiven for what their ancestors did to } \\
\text { [members of your country] during the occupation of Asia, the Sino- } \\
\text { Japanese War and World War II. }\end{array}$ & 1 & 2 & 3 & 4 & 5 & 6 & 7 \\
\hline $\begin{array}{l}\text { 7. It is possible for me to forgive today's Japanese for Japan's past } \\
\text { misdeeds. }\end{array}$ & 1 & 2 & 3 & 4 & 5 & 6 & 7 \\
\hline The following statements are statements about granting forgiveness. & $\begin{array}{l}\text { Stron } \\
\text { disag1 } \\
\Downarrow\end{array}$ & & & $\begin{array}{c}\text { Neutral } \\
\Downarrow\end{array}$ & & & $\begin{array}{l}\text { ngly } \\
\text { gree } \\
\Downarrow\end{array}$ \\
\hline $\begin{array}{l}\text { 1. Granting forgiveness will put the victimized group into an even more } \\
\text { vulnerable position than before. }\end{array}$ & 1 & 2 & 3 & 4 & 5 & 6 & 7 \\
\hline $\begin{array}{l}\text { 2. Granting forgiveness without empowering the victimized group can } \\
\text { lead to a loss of identity for the victimized group. }\end{array}$ & 1 & 2 & 3 & 4 & 5 & 6 & 7 \\
\hline 3. Granting forgiveness is an invitation for crimes to be repeated & 1 & 2 & 3 & 4 & 5 & 6 & 7 \\
\hline 4. Granting forgiveness means losing sympathy as victims & 1 & 2 & 3 & 4 & 5 & 6 & 7 \\
\hline $\begin{array}{l}\text { 5. Granting forgiveness will make the perpetrator believe that now } \\
\text { everything is alright although nothing is really settled. }\end{array}$ & 1 & 2 & 3 & 4 & 5 & 6 & 7 \\
\hline $\begin{array}{l}\text { Please tell us how you feel about interactions between Japanese and } \\
\text { [members of your country]. Please indicate to which degree from 'not at } \\
\text { all' (1) to 'very much' (7) you think each of these question are true. }\end{array}$ & $\begin{array}{l}\text { Not } \\
\text { at all } \\
\Downarrow\end{array}$ & & & & & & $\begin{array}{l}\text { Very } \\
\text { Iuch } \\
\Downarrow\end{array}$ \\
\hline $\begin{array}{l}\text { Do you think today's Japanese people would try to be fair to } \\
\text { [members of your country]? }\end{array}$ & 1 & 2 & 3 & 4 & 5 & 6 & 7 \\
\hline $\begin{array}{l}\text { 2. Would you say that most of the time today's Japanese people try to } \\
\text { be helpful to [members of your country]? }\end{array}$ & 1 & 2 & 3 & 4 & 5 & 6 & 7 \\
\hline $\begin{array}{l}\text { 3. Generally speaking, would you say that most today's Japanese people } \\
\text { can be trusted? }\end{array}$ & 1 & 2 & 3 & 4 & 5 & 6 & 7 \\
\hline
\end{tabular}




\section{Section D: Demographic Information}

1. Are you? $\square$ female $\quad \square$ male

2. What is your age?___years

3. What is your religion (if any)? Please specify:

3a. How important is religion in your life? (please circle a number between 1 and 4 to describe how you feel)

1=not important at all, 2=somewhat important, 3=fairly important, 4=very important

4. Do you actively practice a religion (e.g. going to church, temple)? $\square$ yes $\square$ no

4a. If yes, how often do you practice your religion? Please circle a number between 1 (very seldom) and 7 (daily) that best describes you:
(very seldom) 1
2
$\begin{array}{lll}5 & 6 & 7 \text { (daily) }\end{array}$

5. What is your highest form of education?

$\square$ Secondary school

$\square$ Apprenticeship

$\square$ Polytechnic

$\square$ University

6. What is your occupation?

7. What is your Country of Birth?

8. What is your Nation of Citizenship(s)?

9. How long have you lived in countries outside of this one? years months

10. Does your family have a migration background? $\square$ yes; $\square$ no; $\square$ partially

a. If yes, from which country did your family or parts of your family come from?

b. How long have they been living in [country where participant live

in]?_____ears __ months

11. Often, when people talk about political matters they use terms like "conservative/ rightwing" or "liberal/ left-wing" to describe their views. How would you describe yourself in these terms? Circle a number between 1 (liberal/left-wing) and 7 (conservative/rightwing) that best describes you:

$\begin{array}{llllllll}\begin{array}{c}\text { (Liberal/left-wing) } \\ \text { wing) }\end{array} & 1 & 2 & 3 & 4 & 5 & 6 & 7 \text { (conservative/right- }\end{array}$

a. Other political orientation, please specify:

Thanks a lot for your participation and your help in this research! 


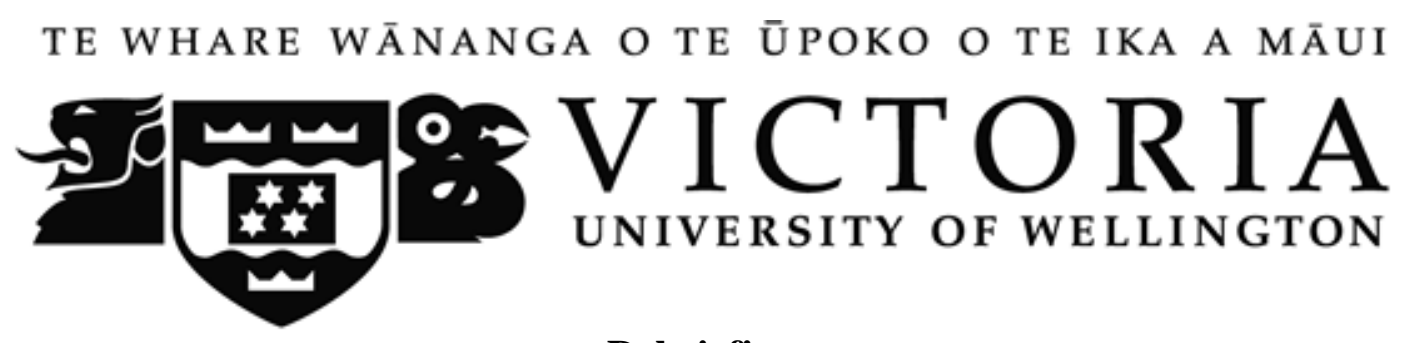

Debriefing

Thank you very much for taking the time and effort to complete this questionnaire and for participating in this research.

The study examines what promotes and hinders forgiveness between once conflicting countries.

Forgiveness between countries may be an alternative means to improve relations between countries that used to be in conflicts. Previous studies have shown that feeling guilty on behalf of your country influences people's willingness to try to repair damage that was caused in the past. Furthermore, empathy (the ability to take another perspective) and trust (the expectation to be treated fairly by another person or group) have an impact on the willingness to forgive. However, forgiveness between countries is still poorly understood. More studies are needed that relate to conflicts that actually happened in order to discover pragmatic means that can improve relations between countries.

In this study we attempted to look at a real post conflict situation between countries and examine forgiveness from a cultural comparison perspective. This has been neglected in previous research. Thus, this perspective can contribute to our understanding about rapprochement between countries after conflict.

Preliminary results of this study will be posted on the website of the Centre for Applied Cross-Cultural Research (http://www.vuw.ac.nz/cacr/) by March 2009. If you have any additional questions please feel free to contact Katja Hanke at Katja.Hanke@vuw.ac.nz

Thank you very much again for participating in this study. 
Appendix B3 -Single-Group CFA

Single-group CFA fit indices for historical closure

\begin{tabular}{llccccc}
\hline \multicolumn{1}{l}{ Country } & $\chi^{2}$ & $\chi^{2} / \mathrm{df}$ & SRMR & RMSEA & GFI & CFI \\
\hline 1. France & 4.55 & 1.52 & .03 & .05 & .99 & .98 \\
2. Poland & 7.55 & 1.89 & .04 & .07 & .99 & .97 \\
3. Russia & 5.89 & 1.18 & .03 & .03 & .99 & .99 \\
4. China & 6.97 & 1.68 & .03 & .06 & .99 & .98 \\
5. Taiwan & 7.12 & 1.43 & .03 & .04 & .99 & .88 \\
6. Philippines & 3.55 & .70 & .02 & .00 & .99 & 1.00 \\
\hline
\end{tabular}

Single-group CFA fit indices for costs of granting forgiveness

\begin{tabular}{|c|c|c|c|c|c|c|}
\hline Country & $\chi^{2}$ & $\chi^{2} / \mathrm{df}$ & SRMR & RMSEA & GFI & $\mathrm{CFI}$ \\
\hline 1. France & 8.26 & 2.07 & .03 & .070 & .98 & .97 \\
\hline 2. Poland & 1.14 & .28 & .01 & .00 & .99 & 1.00 \\
\hline 3. Russia & 4.89 & 1.22 & .03 & .03 & .99 & .99 \\
\hline 4. China & 4.12 & 1.03 & .02 & .01 & .99 & .99 \\
\hline 5. Taiwan & 1.99 & .50 & .02 & .00 & .99 & 1.00 \\
\hline 6. Philippines & 4.00 & 1.00 & .02 & .002 & .99 & 1.00 \\
\hline
\end{tabular}




\title{
Appendix C - Additional Information for Chapter 6
}

\author{
Appendix C1 - Survey Study 4 German context perpetrator version
}

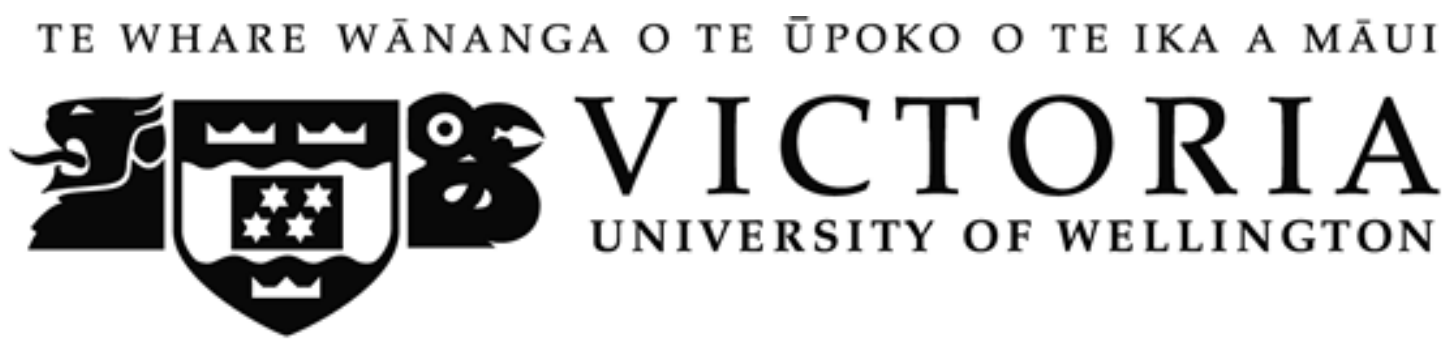

Lieber Teilnehmer, liebe Teilnehmerin,

Sie sind eingeladen, an einer internationalen Studie teilzunehmen, in der es um Ihre Meinung und Wahrnehmung zu den gegenwärtigen Beziehungen zwischen Deutschland und seinen direkten und indirekten europäischen Nachbarländern geht.

Wir wären Ihnen sehr dankbar, wenn Sie sich bereit erklären den folgenden Fragebogen auszufüllen. Ihre Teilnahme ist selbstverständlich vollkommen freiwillig und kann zu jeder Zeit abgebrochen werden.

Durch das vollständige Ausfüllen des Fragebogens stimmen Sie der Teilnahme an diesem Projekt und der Auswertung Ihrer Daten zu.

Auf den folgenden Seiten werden Ihnen einige allgemeine und spezielle Fragen über Politik und internationale Beziehungen gestellt. Bitte beantworten Sie diese Fragen so ehrlich wie möglich und benutzen die angegebenen Antwortskalen. Das Ausfüllen des Fragebogens wird ungefähr 30 Minuten dauern.

- Ihre Antworten bleiben vollständig anonym und nur Wissenschaftler, die direkt in das Projekt eingebunden sind, werden Zugang zu den Daten haben.

- Es wird zu keinem Zeitpunkt möglich sein, Sie in dieser Studie oder in einer Präsentation oder Veröffentlichung persönlich zu identifizieren, da Ihre Daten numerisch kodiert werden.

- Gemäß der Bedingungen einiger wissenschaftlicher Zeitschriften und Organisationen ist es möglich, dass Ihre kodierten Daten anderen kompetenten Wissenschaftlern zugänglich gemacht werden.

- Ihre kodierten Daten könnten in anderen relevanten Studien verwendet werden.

- Eine Kopie Ihrer kodierten Daten wird unter der Obhut von Katja Hanke und Assoc. Prof. James H. Liu sowie anderer involvierter Wissenschaftler aufbewahrt.

Ihre Daten könnten für folgende Zwecke verwendet werden:

- Die Ergebnisse könnten zur Veröffentlichung in einer wissenschaftlichen Zeitschrift eingereicht werden oder auf einer wissenschaftlichen Konferenz präsentiert werden.

- Die Ergebnisse bilden einen Teil einer Doktorarbeit, die zur Bewertung eingereicht wird.

Bitte beantworten Sie die Fragen so ehrlich wie möglich; es gibt keine richtigen oder falschen Antworten.

Wir sind an Ihrer persönlichen Meinung interessiert. Vielen Dank für Ihre Hilfe und Kooperation.

Katja Hanke

\section{Doktorandin}

Victoria University of Wellington

School of Psychology

PO Box 600

Wellington

New Zealand

Email:katja.hanke@vuw.ac.nz
Assoc. Prof. James H. Liu

Associate Professor

Victoria University of Wellington

School of Psychology

PO Box 600

Wellington

New Zealand

Email: james.liu@vuw.ac.nz 


\begin{tabular}{|c|c|c|c|c|c|c|c|}
\hline \multicolumn{8}{|c|}{$\begin{array}{l}\text { Abschnitt B: Wahrnehmung von Geschichte } \\
\text { Wir sind an Ihrer Wahrnehmung der Konsequenzen historischer Ereignisse auf die gegenwärtige } \\
\text { Beziehungen zwischen Deutschland und seinen direkten und indirekten europäischen } \\
\text { Nachbarländern interessiert. Auf den folgenden Seiten werden Ihnen einige Fragen zu Ihrer } \\
\text { Wahrnehmung der Konsequenzen, Ihren Gefühlen und Gedanken zu Hitlers Herrschaft in } \\
\text { Deutschland und Deutschlands Kriegsvergangenheit gestellt. } \\
\text { Bitte beantworten Sie die Fragen so ehrlich wie möglich, in dem Sie die Antwortskala von } 1 \text { (starke } \\
\text { Ablehnung) bis } 7 \text { (starke Zustimmung) verwenden. Bitte lassen Sie keine Fragen aus. Es gibt keine } \\
\text { richtigen oder falschen Antworten. Die beste Antwort ist Ihre eigene Meinung. }\end{array}$} \\
\hline $\begin{array}{l}\text { yeben Sie an, in welchem Maße Sie jede der folgenden } \\
\text { gen ablehnen (1) oder ihr zustimmen (7). }\end{array}$ & \multicolumn{3}{|c|}{$\begin{array}{l}\text { Starke } \\
\text { Ablehnung } \\
\Downarrow\end{array}$} & \multicolumn{2}{|c|}{$\underset{\Downarrow}{\Downarrow}$} & & \\
\hline $\begin{array}{l}\text { iskussionen über das Vergeben und Entschuldigen deutscher } \\
\text { riegsverbrechen sind heute nicht mehr von Bedeutung. }\end{array}$ & 1 & 2 & 3 & 4 & 5 & 6 & 7 \\
\hline $\begin{array}{l}\text { Unmut und die Verbitterung, die mit Deutschlands } \\
\text { riegsverbrechen zusammenhängen, werden auf die nächste } \\
\text { eneration übertragen. }\end{array}$ & 1 & 2 & 3 & 4 & 5 & 6 & 7 \\
\hline $\begin{array}{l}\text { eutzutage ist kein Bedarf mehr vorhanden, über den Schaden } \\
\text { reden, den Deutschland in der Vergangenheit verursacht hat. }\end{array}$ & 1 & 2 & 3 & 4 & 5 & 6 & 7 \\
\hline $\begin{array}{l}\text { eschichten von Überlebenden deutscher Kriegsverbrechen } \\
\text { ermitteln das Gefühl, dass die Wunden heutzutage immer noch } \\
\text { ffen sind. }\end{array}$ & 1 & 2 & 3 & 4 & 5 & 6 & 7 \\
\hline
\end{tabular}

Bitte geben Sie an, in welchem Maße Sie jede der folgenden

Aussagen ablehnen (1) oder ihr zustimmen (7).

Abschnitt C: Gefühle und Gedanken

In den folgenden Aussagen geht es um Deutschlands Verbrechen in der Vergangenheit und um Ihre Einschätzung der Auswirkungen dieser Verbrechen auf das Deutschland von heute. Bitte geben Sie an, in welchem Maße Sie jede der folgenden Aussagen ablehnen (1) oder ihr zustimmen (7). Bitte lassen Sie keine Aussagen aus. Es gibt keine richtigen oder falschen Antworten. Die beste Antwort ist Ihre eigene Meinung.

\begin{tabular}{|c|c|c|c|c|c|c|c|}
\hline \multirow[b]{2}{*}{$\begin{array}{l}\text { 1. Ich fühle Bedauern für Deutschlands Gräueltaten in der } \\
\text { Vergangenheit an anderen Gruppen. }\end{array}$} & \multicolumn{2}{|c|}{$\begin{array}{l}\text { Starke } \\
\text { Ablehnung } \\
\Downarrow\end{array}$} & \multicolumn{3}{|c|}{$\underset{\substack{\text { Neutral } \\
\Downarrow}}{\Downarrow}$} & \multicolumn{2}{|c|}{$\begin{array}{r}\text { Starke } \\
\text { Zustimmun } \\
\text { g }\end{array}$} \\
\hline & 1 & 2 & 3 & 4 & 5 & 6 & 7 \\
\hline $\begin{array}{l}\text { 2. Ich fühle mich schuldig für die negativen Dinge, die meine } \\
\text { Vorfahren anderen Gruppen angetan haben. }\end{array}$ & 1 & 2 & 3 & 4 & 5 & 6 & 7 \\
\hline $\begin{array}{l}\text { 3. Ich fühle Reue für einige von den Dingen, die Deutschland anderen } \\
\text { Gruppen in der Vergangenheit angetan hat. }\end{array}$ & 1 & 2 & 3 & 4 & 5 & 6 & 7 \\
\hline $\begin{array}{l}\text { 4. Ich glaube, dass ich den Schaden wiedergutmachen sollte, den } \\
\text { Deutschland bei Anderen in der Vergangenheit verursacht hat. }\end{array}$ & 1 & 2 & 3 & 4 & 5 & 6 & 7 \\
\hline $\begin{array}{l}\text { 5. Ich kann mich sehr leicht schuldig für die schlimmen Folgen } \\
\text { fühlen, die Deutschland in der Vergangenheit hervorgebracht hat. }\end{array}$ & 1 & 2 & 3 & 4 & 5 & 6 & 7 \\
\hline $\begin{array}{l}\text { 1. Ich schäme mich, weil Deutschland in der Vergangenheit so viele } \\
\text { Verbrechen an anderen europäischen Nationen begangen hat. }\end{array}$ & 1 & 2 & 3 & 4 & 5 & 6 & 7 \\
\hline $\begin{array}{l}\text { 2. Ich schäme mich für das, was unsere Urgroß- und Großeltern } \\
\text { während der Hitler-Diktatur und während des Zweiten Weltkrieges } \\
\text { getan haben. }\end{array}$ & 1 & 2 & 3 & 4 & 5 & 6 & 7 \\
\hline 3. Ich schäme mich, wenn Ausländer den Holocaust erwähnen. & 1 & 2 & 3 & 4 & 5 & 6 & 7 \\
\hline $\begin{array}{l}\text { 4. Verbrechen, die von Deutschland während der Hitler-Diktatur und } \\
\text { während des Zweiten Weltkrieges verübt wurden, sind ein großes } \\
\text { schwarzes Schandmal in der Geschichte Deutschlands. }\end{array}$ & 1 & 2 & 3 & 4 & 5 & 6 & 7 \\
\hline
\end{tabular}




\begin{tabular}{|c|c|c|c|c|c|c|c|c|}
\hline & $\begin{array}{l}\text { Öffentliche Diskussionen über die vergangenen Verbrechen meiner } \\
\text { Vorfahren bereiten mir Schamgefühle. }\end{array}$ & 1 & 2 & 3 & 4 & 5 & 6 & 7 \\
\hline 1. & $\begin{array}{l}\text { Die deutsche Regierung hat durch das Eingestehen der während } \\
\text { des Zweiten Weltkriegs begangenen Gewalttaten einen } \\
\text { Gesichtsverlust der deutschen Bevölkerung hervorgerufen. }\end{array}$ & 1 & 2 & 3 & 4 & 5 & 6 & 7 \\
\hline 2. & $\begin{array}{l}\text { Das Eingestehen der während des Zweiten Weltkriegs begangenen } \\
\text { Gewalttaten durch die deutsche Regierung hatte einen nicht } \\
\text { wiedergutzumachenden Gesichtsverlust der deutschen Bevölkerung } \\
\text { zur Folge. }\end{array}$ & 1 & 2 & 3 & 4 & 5 & 6 & 7 \\
\hline 3. & $\begin{array}{l}\text { Es ist verständlich, dass Deutsche heutzutage über offizielle } \\
\text { Entschuldigungen für den deutschen Aggressionskrieg besorgt } \\
\text { sind, weil diese in der Vergangenheit einen Gesichtsverlust für die } \\
\text { deutsche Bevölkerung bedeuteten. }\end{array}$ & 1 & 2 & 3 & 4 & 5 & 6 & 7 \\
\hline & $\begin{array}{l}\text { Die Bitte um Vergebung durch Vertreter/innen des deutschen } \\
\text { Staates hatte einen Gesichtsverlust für die gesamte Nation zur } \\
\text { Folge. }\end{array}$ & 1 & 2 & 3 & 4 & 5 & 6 & 7 \\
\hline
\end{tabular}

Einstellungen zu Wiedergutmachung/Reparationszahlungen. Bitte geben Sie an, in welchem Maße Sie die folgenden Aussagen ablehnen (1) oder ihnen zustimmen (7).

1. Es ist wichtig, dass ehemalige Opfer von Deutschlands Kriegsverbrechen unterstützt werden, wenn sie Forderungen stellen.

2. Ich zeige meine Bereitschaft, ehemalige Opfer von Deutschlands Kriegsverbrechen zu unterstützen, indem ich Buttons/Aufnäher mit politischen Aussagen trage.

3. Ich bin bereit, mich aktiv in einer Institution zu engagieren, die für die Fortsetzung der Ausgleichszahlungen für Deutschlands Kriegsverbrechen sorgt.

4. Ich bin bereit, Leute für Unterschriftensammlungen anzuwerben, die die Forderungen für die Fortsetzung von

Ausgleichszahlungen unterstützen und an die deutsche Regierung gerichtet sind.

5. Ich denke, ehemalige Opfer des Zweiten Weltkrieges verdienen für das, was ihnen während des Krieges passiert ist, eine Art von Wiedergutmachung von Seiten Deutschlands.

\begin{tabular}{|c|c|c|c|c|c|c|}
\hline \multicolumn{3}{|l}{$\begin{array}{l}\text { Starke } \\
\text { Ablehnung } \\
\Downarrow\end{array}$} & \multicolumn{4}{|c}{$\begin{array}{r}\text { Neutral } \\
\Downarrow\end{array}$} \\
\hline 1 & 2 & 3 & 4 & 5 & 6 & $\begin{array}{r}\text { Starke } \\
\text { Zustimmun } \\
\text { g }\end{array}$ \\
\hline 1 & 2 & 3 & 4 & 5 & 6 & 7 \\
\hline 1 & 2 & 3 & 4 & 5 & 6 & 7 \\
\hline 1 & 2 & 3 & 4 & 5 & 6 & 7 \\
\hline
\end{tabular}

\begin{tabular}{|c|c|c|c|c|c|c|c|}
\hline \multirow{2}{*}{$\begin{array}{l}\text { Die folgenden Aussagen sind Aussagen über die Bitte um Vergebung } \\
\text { zwischen Gruppen. } \\
\text { 1. } \\
\begin{array}{l}\text { Die Bitte um Vergebung kann die Kommunikation zwischen } \\
\text { ehemals in Konflikt stehenden Gruppen einleiten. }\end{array} \\
\end{array}$} & \multicolumn{2}{|c|}{$\begin{array}{l}\text { Starke } \\
\text { Ablehnung } \\
\Downarrow\end{array}$} & \multicolumn{3}{|c|}{$\begin{array}{c}\text { Neutral } \\
\Downarrow\end{array}$} & \multicolumn{2}{|c|}{$\begin{array}{r}\text { Starke } \\
\text { Zustimmun } \\
\text { g } \\
\Downarrow\end{array}$} \\
\hline & 1 & 2 & 3 & 4 & 5 & 6 & 7 \\
\hline $\begin{array}{l}\text { 2. Der erste Schritt zu sozialer Harmonie zwischen Gruppen könnte } \\
\text { die Bitte um Vergebung sein. }\end{array}$ & 1 & 2 & 3 & 4 & 5 & 6 & 7 \\
\hline $\begin{array}{l}\text { 3. Ein Gesuch um Vergebung ist gleichzusetzen mit einem } \\
\text { Eingeständnis der Tatsache, dass die verletzenden Taten wirklich } \\
\text { passiert sind. }\end{array}$ & 1 & 2 & 3 & 4 & 5 & 6 & 7 \\
\hline
\end{tabular}




\begin{tabular}{|c|c|c|c|c|c|c|c|}
\hline $\begin{array}{l}\text { Bitte geben Sie an, in welchem Maße Sie jede der folgenden Aussagen } \\
\text { ablehnen (1) oder ihr zustimmen (7). }\end{array}$ & \multicolumn{2}{|c|}{$\begin{array}{l}\text { Starke } \\
\text { Ablehnun } \\
\text { g } \\
\Downarrow\end{array}$} & \multicolumn{2}{|c|}{$\underset{\Downarrow}{\text { Neutral }}$} & \multicolumn{3}{|c|}{$\begin{array}{r}\text { Starke } \\
\text { Zustimmun } \\
\text { g } \\
\Downarrow\end{array}$} \\
\hline $\begin{array}{l}\text { 1. Ich versuche mir vorzustellen, was andere Europäer während des } \\
\text { Zweiten Weltkrieges durchmachen mussten. }\end{array}$ & 1 & 2 & 3 & 4 & 5 & 6 & 7 \\
\hline $\begin{array}{l}\text { 2. Manchmal denke ich darüber nach, wie sich andere Europäer } \\
\text { während des Zweiten Weltkrieges gefühlt haben. }\end{array}$ & 1 & 2 & 3 & 4 & 5 & 6 & 7 \\
\hline $\begin{array}{l}\text { 3. Ich versuche mir die Dinge, die während des Zweiten Weltkrieges } \\
\text { geschehen sind, aus der Perspektive anderer Europäer zu betrachten. }\end{array}$ & 1 & 2 & 3 & 4 & 5 & 6 & 7 \\
\hline $\begin{array}{l}\text { 4. Normalerweise bin ich in der Lage, die Ansicht anderer Europäer zu } \\
\text { verstehen. }\end{array}$ & 1 & 2 & 3 & 4 & 5 & 6 & 7 \\
\hline $\begin{array}{l}\text { 5. Bitten tragen Sie im nebenstehenden leeren Feld den oder die } \\
\text { Name(n) der Europäischen Nation(en) ein, die Sie im Kopf hatten, } \\
\text { während Sie antworteten: }\end{array}$ & & & & & & & \\
\hline
\end{tabular}

Bitte teilen Sie uns mit, wie Sie sich den Interaktionen zwischen deutschen und anderen Europäern gegenüber fühlen. Bitte geben Sie an, in welchem Maße (von ' 1 ' (überhaupt nicht) bis ' 7 ' (sehr viel)) Sie denken, dass jede der folgenden Fragen der Wahrheit entspricht.

\begin{tabular}{|c|c|c|c|c|c|c|c|c|}
\hline 1. & $\begin{array}{l}\text { Denken Sie, dass die meisten Europäer versuchen würden, fair zu } \\
\text { sein? }\end{array}$ & 1 & 2 & 3 & 4 & 5 & 6 & 7 \\
\hline 2. & $\begin{array}{l}\text { Würden Sie sagen, dass andere Europäer meistens versuchen, } \\
\text { hilfsbereit zu sein? }\end{array}$ & 1 & 2 & 3 & 4 & 5 & 6 & 7 \\
\hline 3. & $\begin{array}{l}\text { Würden Sie im Allgemeinen sagen, dass man den meisten Europäern } \\
\text { vertrauen kann? }\end{array}$ & 1 & 2 & 3 & 4 & 5 & 6 & 7 \\
\hline 4. & $\begin{array}{l}\text { Bitten tragen Sie im nebenstehenden leeren Feld den oder die } \\
\text { Name(n) der Europäischen Nation(en) ein, die Sie im Kopf hatten, } \\
\text { während Sie antworteten: }\end{array}$ & & & & & & & \\
\hline
\end{tabular}




\section{Abschnitt D: Demographische Informationen}

1. Sind Sie? $\square$ weiblich $\quad \square$ männlich

2. Wie alt sind Sie? Jahre

3. Welcher Religion gehören Sie an (falls Sie einer angehören)?

3a. Wie wichtig ist Religion in Ihrem Leben? (Bitte markieren Sie die entsprechende Ziffer)

1 = überhaupt nicht wichtig, 2 = etwas wichtig, 3 = ziemlich wichtig, $4=$ sehr wichtig

4. Praktizieren Sie aktiv eine Religion (z.B. in die Kirche gehen)? $\square$ ja $\square$ nein 4a. Falls ja, wie oft tun Sie dies? (Bitte markieren Sie eine Ziffer zwischen 1 (sehr selten) und 7 (täglich):

$\begin{array}{lllllll}\text { (sehr selten) } 1 & 2 & 3 & 4 & 5 & 6 & 7 \text { (täglich) }\end{array}$

5. Ihr höchster Bildungsabschluss?

$\square$ Ohne Abschluss

$\square$ Hauptschul-Abschluss

Realschul-Abschluss

Fachhochschulreife

Hochschulreife (Abitur)

$\square$ Lehre / Fachschule

$\square$ Fachhochschul- / Universitäts-Abschluss

6. Erlernter Beruf / Beschäftigung:

7. In welchem Land wurden Sie

geboren?

7a. In welchem Bundesland wurden Sie geboren?

8. Welche Staatsbürgerschaft besitzen Sie?

9. Wie lange haben Sie insgesamt im Ausland gelebt? Jahre Monate

10. Hat Ihre Familie einen Migrationshintergund? $\square$ ja; $\square$ nein; $\square$ teilweise

10a. Falls ja, aus welchem Land stammt Ihre Familie /ein Teil Ihrer Familie?

10b. Wie lange leben diese / leben Sie bereits in Deutschland? Jahre Monate 
11. Wenn über politische Themen gesprochen wird, verwenden die Leute oft Ausdrücke wie „links“ oder „rechts“, um ihre Ansichten zu beschreiben. Wie würden Sie sich selbst anhand dieser Ausdrücke einschätzen? Kreisen Sie die Nummer zwischen 1 (links) und 7 (rechts) ein, die Sie am besten beschreibt:

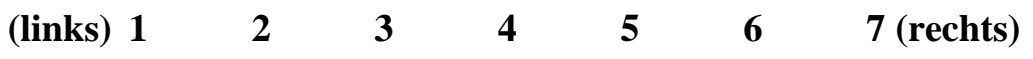

11a Andere politische Orientierung:

12. Welche Partei würden Sie bei der nächsten Bundestagswahl wählen?

\section{Herzlichen Dank für die Unterstützung dieses Projekts durch Ihre Teilnahme!}




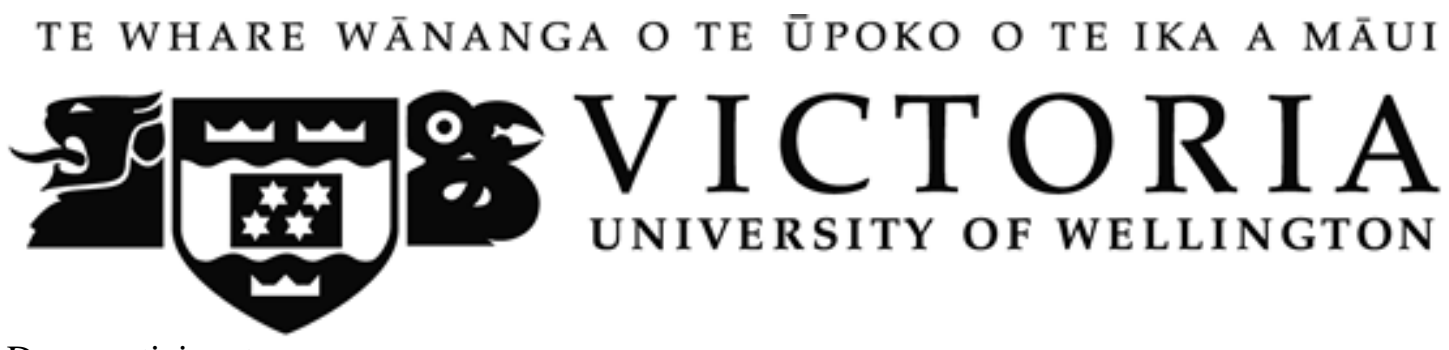

Dear participant,

You are invited to participate in an international survey on your views and perceptions of current relations between Japan and her Asian neighbours.

We would appreciate it, if you would be willing to fill out the following questionnaire. Your participation is entirely voluntary and you are free to withdraw at any stage before you complete and hand back the questionnaire to us. You grant your consent to participate in this research by filling in the questionnaire.

On the following pages, you will see some questions about some general and specific statements about politics and international relations. Please answer these questions as honestly as possible using the provided rating scales. It will take about 30 minutes to fill out the questionnaire.

- Your answers are completely anonymous and only investigators directly involved in the project will have access to the data.

- You will never be personally identified in this research project or in any presentation or publication.

- The information you provide will be coded by number only. In accordance with the requirements of some scientific journals and organisations, your coded data may be shared with other competent researchers.

- Your coded data may be used in other, related studies.

- A copy of the coded data will remain in the custody of investigators.

Please do not write your name or any identifying information on the questionnaire itself.

The data you provide may be used for one or more of the following purposes:

- The overall findings may be submitted for publication in a scientific journal, or presented at scientific conferences.

- The overall findings may form part of a PhD thesis that will be submitted for assessment.

Please remember, there are no right or wrong answers. We are interested in your personal opinion on these issues.

Thank you very much for your help and cooperation.

Katja Hanke

PhD Student

Victoria University of Wellington

School of Psychology

PO Box 600

Wellington

New Zealand

Email:katja.hanke@vuw.ac.nz
Assoc. Prof. James H. Liu

Associate Professor

Victoria University of Wellington

School of Psychology

PO Box 600

Wellington

New Zealand

Email:james.liu@vuw.ac.nz 


\section{Section A: Identity}

Please indicate to which degree you disagree (1) or agree (7) with each of the following statements.

There are no right or wrong answers. The best answer is your own opinion

\begin{tabular}{|c|c|c|c|c|c|c|c|}
\hline \multirow{2}{*}{$\begin{array}{l}\text { National Identity } \\
\text { 1. I feel a bond with Japanese people. }\end{array}$} & \multicolumn{3}{|c|}{$\begin{array}{l}\text { Strongly } \\
\text { disagree } \\
\Downarrow\end{array}$} & eutr: & & \multicolumn{2}{|c|}{$\begin{array}{r}\text { Strongly } \\
\text { agree } \\
\Downarrow\end{array}$} \\
\hline & 1 & 2 & 3 & 4 & 5 & 6 & 7 \\
\hline 2. I feel solidarity with Japanese people. & 1 & 2 & 3 & 4 & 5 & 6 & 7 \\
\hline 3. I feel committed to Japanese people. & 1 & 2 & 3 & 4 & 5 & 6 & 7 \\
\hline 4. I am glad to be Japanese. & 1 & 2 & 3 & 4 & 5 & 6 & 7 \\
\hline 5. I think that Japanese people have a lot to be proud of. & 1 & 2 & 3 & 4 & 5 & 6 & 7 \\
\hline 6. It is pleasant to be Japanese. & 1 & 2 & 3 & 4 & 5 & 6 & 7 \\
\hline 7. I often think about the fact that I am Japanese. & 1 & 2 & 3 & 4 & 5 & 6 & 7 \\
\hline 8. The fact that I am Japanese is an important part of my identity. & 1 & 2 & 3 & 4 & 5 & 6 & 7 \\
\hline 9. Being Japanese is an important part of how I see myself. & 1 & 2 & 3 & 4 & 5 & 6 & 7 \\
\hline 10. I am similar to many Japanese people. & 1 & 2 & 3 & 4 & 5 & 6 & 7 \\
\hline 11. Japanese people have a lot in common with each other. & 1 & 2 & 3 & 4 & 5 & 6 & 7 \\
\hline 12. Japanese people are very similar to each other. & 1 & 2 & 3 & 4 & 5 & 6 & 7 \\
\hline Global Consciousness & \multicolumn{3}{|c|}{$\begin{array}{l}\text { Strongly } \\
\text { disagree } \\
\Downarrow \\
\end{array}$} & $\begin{array}{r}\text { Neut } \\
\Downarrow \\
\end{array}$ & & \multicolumn{2}{|c|}{$\begin{array}{r}\text { Strongly } \\
\text { agree } \\
\Downarrow\end{array}$} \\
\hline $\begin{array}{l}\text { 1. I think of myself as a citizen of the world rather than of one } \\
\text { particular country. }\end{array}$ & 1 & 2 & 3 & 4 & 5 & 6 & 7 \\
\hline $\begin{array}{l}\text { 2. Citizens of each country should look after themselves instead of } \\
\text { worrying about people from other parts of the world. }\end{array}$ & 1 & 2 & 3 & 4 & 5 & 6 & 7 \\
\hline $\begin{array}{l}\text { 3. I think of myself as a loyal citizen of my own country rather than } \\
\text { as a citizen of the world. }\end{array}$ & 1 & 2 & 3 & 4 & 5 & 6 & 7 \\
\hline 4. People from each country should mind their own business. & 1 & 2 & 3 & 4 & 5 & 6 & 7 \\
\hline $\begin{array}{l}\text { 5. I identify with the human race more than any particular } \\
\text { nationality. }\end{array}$ & 1 & 2 & 3 & 4 & 5 & 6 & 7 \\
\hline $\begin{array}{l}\text { 6. Being a good citizen of the world is an important part of how I see } \\
\text { myself. }\end{array}$ & 1 & 2 & 3 & 4 & 5 & 6 & 7 \\
\hline
\end{tabular}




\section{Section B: Perception of history}

We are interested in your perception of the consequences of historical events on current relations between Japan and her Asian neighbours. On the following pages, you will be asked a number of questions about consequences, perceptions, emotions and thoughts about Japan's colonial and wartime past.

Please answer these questions as honestly as possible using the provided rating scales. There are no right or wrong answers. The best answer is your own opinion.

\begin{tabular}{|c|c|c|c|c|c|c|c|}
\hline $\begin{array}{l}\text { Please indicate to which degree you disagree (1) or agree (7) with each of } \\
\text { the following statements. }\end{array}$ & \multicolumn{2}{|c|}{$\begin{array}{l}\text { Strongly } \\
\text { disagree } \\
\Downarrow\end{array}$} & \multicolumn{3}{|c|}{$\underset{\Downarrow}{\text { Neutral }}$} & \multicolumn{2}{|c|}{$\begin{array}{r}\text { Strongly } \\
\text { agree } \\
\Downarrow\end{array}$} \\
\hline $\begin{array}{l}\text { 1. Discussions about apologizing and forgiving related to Japan's } \\
\text { harmful past actions towards other Asians are not important any } \\
\text { more. }\end{array}$ & 1 & 2 & 3 & 4 & 5 & 6 & 7 \\
\hline $\begin{array}{l}\text { 2. Grievances and resentment related to Japan's harmful past actions } \\
\text { towards other Asians will be carried to the next generation. }\end{array}$ & 1 & 2 & 3 & 4 & 5 & 6 & 7 \\
\hline $\begin{array}{l}\text { 3. Today there is no need to talk so much about the harm committed by } \\
\text { Japan in the past. }\end{array}$ & 1 & 2 & 3 & 4 & 5 & 6 & 7 \\
\hline $\begin{array}{l}\text { 4. Other Asians' stories of surviving Japan's harmful past actions make } \\
\text { me feel like the wounds are still open today. }\end{array}$ & 1 & 2 & 3 & 4 & 5 & 6 & 7 \\
\hline
\end{tabular}

Section C: Emotions \& Thoughts

The following statements are about the relation between past misdeeds of Japan and Japanese people today Please indicate to which degree you disagree (1) or agree (7) with each of the following statements. There are no right or wrong answers. The best answer is your own opinion.

\begin{tabular}{|c|c|c|c|c|c|c|c|}
\hline $\begin{array}{l}\text { Please tell us to which degree you disagree (' } 1 \text { ') or agree ('7') with each } \\
\text { statement }\end{array}$ & \multicolumn{2}{|c|}{$\begin{array}{l}\text { Strongly } \\
\text { disagree } \\
\Downarrow\end{array}$} & \multicolumn{3}{|c|}{$\underset{\Downarrow}{\text { Neutral }}$} & \multicolumn{2}{|c|}{$\begin{array}{r}\text { Strongly } \\
\text { agree } \\
\Downarrow\end{array}$} \\
\hline 1. I feel regret for Japan's harmful past actions towards other groups. & 1 & 2 & 3 & 4 & 5 & 6 & 7 \\
\hline I feel guilty about the negative my ancestors did to other groups. & 1 & 2 & 3 & 4 & 5 & 6 & 7 \\
\hline $\begin{array}{l}\text { 3. I feel regret for some of the things Japan did to other groups in the } \\
\text { past. }\end{array}$ & 1 & 2 & 3 & 4 & 5 & 6 & 7 \\
\hline $\begin{array}{l}\text { 4. I believe that I should repair damage caused to others by Japan in } \\
\text { the past. }\end{array}$ & 1 & 2 & 3 & 4 & 5 & 6 & 7 \\
\hline $\begin{array}{l}\text { 5. I can easily feel guilty for the bad outcomes brought about by } \\
\text { Japanese people in the past. }\end{array}$ & 1 & 2 & 3 & 4 & 5 & 6 & 7 \\
\hline $\begin{array}{l}\text { Please tell us to which degree you disagree (' } 1 \text { ') or agree ('7') with each } \\
\text { statement }\end{array}$ & $\begin{array}{l}\text { Stro } \\
\text { disa } \\
\Downarrow\end{array}$ & & & Neut & & & $\begin{array}{l}\text { gly } \\
\text { ree } \\
\Downarrow\end{array}$ \\
\hline $\begin{array}{l}\text { 1. I feel ashamed because Japan committed so many misdeeds against } \\
\text { other Asian nations in the past. }\end{array}$ & 1 & 2 & 3 & 4 & 5 & 6 & 7 \\
\hline $\begin{array}{l}\text { 2. I feel ashamed about what our (great) grandparents did during the } \\
\text { occupation of Asia, the Sino-Japanese War and World War II. }\end{array}$ & 1 & 2 & 3 & 4 & 5 & 6 & 7 \\
\hline $\begin{array}{l}\text { 3. I feel ashamed when foreigners mention the Nanjing massacre and } \\
\text { the Comfort Women Issue. }\end{array}$ & 1 & 2 & 3 & 4 & 5 & 6 & 7 \\
\hline $\begin{array}{l}\text { 4. Misdeeds committed by Japanese during the occupation of Asia } \\
\text { and World War II are a big black mark in Japan's history. }\end{array}$ & 1 & 2 & 3 & 4 & 5 & 6 & 7 \\
\hline $\begin{array}{l}\text { 5. Public discussions about past misdeeds make me feel ashamed of } \\
\text { my ancestors' past actions. }\end{array}$ & 1 & 2 & 3 & 4 & 5 & 6 & 7 \\
\hline
\end{tabular}




\begin{tabular}{|c|c|c|c|c|c|c|c|}
\hline \multirow{2}{*}{$\begin{array}{l}\begin{array}{l}\text { Please tell us to which degree you disagree (' } 1 \text { ') or agree ('7') with each } \\
\text { statement }\end{array} \\
1 . \quad \begin{array}{l}\text { If Japan's government acknowledges the harm done during the } \\
\text { occupation of Asia and World War II, Japanese people will lose face. }\end{array}\end{array}$} & \multicolumn{3}{|c|}{$\begin{array}{l}\text { Strongly } \\
\text { disagree } \\
\Downarrow \\
\end{array}$} & \multicolumn{2}{|c|}{$\underset{\Downarrow}{\text { Neutral }}$} & \multicolumn{2}{|c|}{$\begin{array}{r}\text { Strongly } \\
\text { agree } \\
\Downarrow\end{array}$} \\
\hline & 1 & 2 & 3 & 4 & 5 & 6 & 7 \\
\hline $\begin{array}{l}\text { 2. If Japan's government acknowledges the harm done during the } \\
\text { occupation of Asia and World War II, the extent of face loss for } \\
\text { Japanese people will be irreparable. }\end{array}$ & 1 & 2 & 3 & 4 & 5 & 6 & 7 \\
\hline $\begin{array}{l}\text { 3. It is understandable that Japanese people would be concerned about a } \\
\text { loss of face, if official apologies to another country for Japan's war } \\
\text { of aggression are issued. }\end{array}$ & 1 & 2 & 3 & 4 & 5 & 6 & 7 \\
\hline $\begin{array}{l}\text { 4. If the Japanese prime minister seeks forgiveness from other Asian } \\
\text { countries, he risks a loss of face for the whole nation. }\end{array}$ & 1 & 2 & 3 & 4 & 5 & 6 & 7 \\
\hline $\begin{array}{l}\text { Attitudes towards compensation. } \\
\text { Please tell us to which degree you disagree ('1') or agree ('7') with each } \\
\text { statement. }\end{array}$ & & & & $\begin{array}{c}\text { Neutr: } \\
\Downarrow\end{array}$ & & & \\
\hline $\begin{array}{l}\text { 1. It's important to support claims of compensation of former overseas } \\
\text { Asian (non-Japanese) victims of Japan's harmful past actions during } \\
\text { the occupation of Asia and World War II. }\end{array}$ & 1 & 2 & 3 & 4 & 5 & 6 & 7 \\
\hline $\begin{array}{l}\text { 2. I am willing to show my support for former overseas Asian (non- } \\
\text { Japanese) victims of Japan's warfare through wearing badges with } \\
\text { political statements. }\end{array}$ & 1 & 2 & 3 & 4 & 5 & 6 & 7 \\
\hline $\begin{array}{l}\text { 3. I am willing to be actively involved in an institution by running } \\
\text { information stalls that supports the demand for an official } \\
\text { acknowledgement of Japan's harmful past actions towards other } \\
\text { (non-Japanese) Asians. }\end{array}$ & 1 & 2 & 3 & 4 & 5 & 6 & 7 \\
\hline $\begin{array}{l}\text { I. I am willing to recruit people to sign petitions that supports } \\
\text { compensation claims addressed to Japan's government. }\end{array}$ & 1 & 2 & 3 & 4 & 5 & 6 & 7 \\
\hline $\begin{array}{l}\text { I. I think that former overseas Asian (non-Japanese) victims of Japan's } \\
\text { harmful past actions deserve some form of compensation from Japan. }\end{array}$ & 1 & 2 & 3 & 4 & 5 & 6 & 7 \\
\hline $\begin{array}{l}\text { The following statements are statements about seeking forgiveness. } \\
\text { Please indicate by circling a number to which degree you disagree (1) or } \\
\text { agree (7) with each of the following statements. }\end{array}$ & $\begin{array}{l}\text { Stron } \\
\text { disag } \\
\Downarrow\end{array}$ & & & $\begin{array}{c}\text { Neutra } \\
\Downarrow\end{array}$ & & & \\
\hline $\begin{array}{l}\text { 1. Seeking forgiveness can open up communication between once } \\
\text { conflicting groups. }\end{array}$ & 1 & 2 & 3 & 4 & 5 & 6 & 7 \\
\hline $\begin{array}{l}\text { 2. Seeking forgiveness may be the first step towards social harmony } \\
\text { between groups. }\end{array}$ & 1 & 2 & 3 & 4 & 5 & 6 & 7 \\
\hline $\begin{array}{l}\text { 3. A request for forgiveness equals recognizing that the harm done } \\
\text { actually happened. }\end{array}$ & 1 & 2 & 3 & 4 & 5 & 6 & 7 \\
\hline $\begin{array}{l}\text { 4. To ask for forgiveness of past misdeeds means to lose pride in } \\
\text { one's own group identity. }\end{array}$ & 1 & 2 & 3 & 4 & 5 & 6 & 7 \\
\hline $\begin{array}{l}\text { Please indicate to which degree you disagree (1) or agree (7) with each } \\
\text { of the following statements. }\end{array}$ & $\begin{array}{l}\text { Strong } \\
\text { disagr } \\
\Downarrow\end{array}$ & & & eutral & & & \\
\hline $\begin{array}{l}\text { 1. I try to imagine what other Asians have gone through during } \\
\text { World War II. }\end{array}$ & 1 & 2 & 3 & 4 & 5 & 6 & 7 \\
\hline $\begin{array}{l}\text { 2. I sometimes think about how other Asians might have felt during } \\
\text { World War II. }\end{array}$ & 1 & 2 & 3 & 4 & 5 & 6 & 7 \\
\hline $\begin{array}{l}\text { 3. I am trying to look at things that happened during World War II } \\
\text { from the perspective of other Asians. }\end{array}$ & 1 & 2 & 3 & 4 & 5 & 6 & 7 \\
\hline $\begin{array}{l}\text { 4. Usually, I am able to understand the other Asians' point of view } \\
\text { regarding World War II. }\end{array}$ & 1 & 2 & 3 & 4 & 5 & 6 & 7 \\
\hline $\begin{array}{l}\text { 5. Please fill in the name of the Asian nation(s) you had in mind } \\
\text { while answering: }\end{array}$ & & & & & & & \\
\hline
\end{tabular}




\begin{tabular}{|c|c|c|c|c|c|c|c|}
\hline \multirow{2}{*}{$\begin{array}{l}\text { Please tell us how you feel about interactions between Japanese } \\
\text { and other Asians. Please indicate to which degree from 'not at all' } \\
\text { (1) to 'very much' (7) you think each of these question are true. } \\
\text { 1. Do you think most other Asians would try to be fair? }\end{array}$} & \multicolumn{2}{|c|}{$\begin{array}{l}\text { Not } \\
\text { at all } \\
\Downarrow\end{array}$} & \multicolumn{3}{|c|}{$\begin{array}{c}\text { Somewhat } \\
\Downarrow\end{array}$} & \multicolumn{2}{|c|}{$\begin{array}{l}\text { Very } \\
\text { much } \\
\end{array}$} \\
\hline & 1 & 2 & 3 & 4 & 5 & 6 & 7 \\
\hline $\begin{array}{l}\text { 2. Would you say that most of the time other Asians try to be } \\
\text { helpful? }\end{array}$ & 1 & 2 & 3 & 4 & 5 & 6 & 7 \\
\hline $\begin{array}{l}\text { 3. Generally speaking, would you say that most other Asians can } \\
\text { be trusted? }\end{array}$ & 1 & 2 & 3 & 4 & 5 & 6 & 7 \\
\hline $\begin{array}{l}\text { 4. Please fill in the name of the Asian nation(s) you had in mind } \\
\text { while answering: }\end{array}$ & & & & & & & \\
\hline
\end{tabular}




\section{Section D: Demographic Information}

1. Are you? $\square$ female $\quad \square$ male

2. What is your age? years

3. What is your religion (if any)? Please specify:

3a. How important is religion in your life? (please circle a number between 1 and 4 to describe how you feel)

$1=$ not important at all, $2=$ somewhat important, $3=$ fairly important, $4=$ very important

4. Do you actively practice a religion (e.g. going to church, temple)? $\square$ yes $\square$ no

4a. If yes, how often do you practice your religion? Please circle a number between 1 (very seldom) and 7 (daily) that best describes you:
(very seldom) 1
23
$4 \quad 5$
$6 \quad 7$ (daily)

5. What is your highest form of education?

$\square$ Secondary school

$\square$ Apprenticeship

$\square$ Polytechnic

$\square$ University

6. What is your occupation?

7. What is your Country of Birth?

8. What is your Nation of Citizenship(s)?

9. How long have you lived in countries outside of this one? years months

10. Does your family have a migration background? $\square$ yes; $\square$ no; $\square$ partially

c. If yes, from which country did your family or parts of your family come from?

d. How long have they been living in Japan? years months

11. Often, when people talk about political matters they use terms like "conservative/ rightwing" or "liberal/ left-wing" to describe their views. How would you describe yourself in these terms? Circle a number between 1 (liberal/left-wing) and 7 (conservative/rightwing) that best describes you:

$\begin{array}{lllllll}\text { (Liberal/left-wing) } 1 & 2 & 3 & 4 & 5 & 6 & \text { 7(conservative/right-wing) }\end{array}$

a) Other political orientation, please specify:

Thanks a lot for your participation and your help in this research! 